\title{
Spinodal nanodecomposition in semiconductors doped with transition metals
}

\author{
T. Dietl, ${ }^{1,2,3}$ K. Sato, ${ }^{4,5,}$, T. Fukushima, ${ }^{6}$ A. Bonanni, ${ }^{7,}$ 円 M. Jamet, ${ }^{8}$ A. Barski, ${ }^{8}$ S. Kuroda, ${ }^{9}$ M. Tanaka, ${ }^{10}$ \\ Pham Nam Hai, ${ }^{11}$ and H. Katayama-Yoshida ${ }^{6,5}$ \\ ${ }^{1}$ Institute of Physics, Polish Academy of Sciences, PL-02-668 Warszawa, Poland \\ ${ }^{2}$ Institute of Theoretical Physics, Faculty of Physics, University of Warsaw, PL-02-093 Warszawa, Poland \\ ${ }^{3}$ WPI-Advanced Institute for Materials Research (WPI-AIMR), Tohoku University, Sendai 980-8577, Japan \\ ${ }^{4}$ Graduate School of Engineering, Osaka University, Suita, Osaka 565-0871, Japan \\ ${ }^{5}$ Institute for NanoScience Design, Osaka University, Toyonaka, Osaka 560-8531, Japan \\ ${ }^{6}$ Graduate School of Engineering Science, Osaka University, Toyonaka, Osaka 560-8531, Japan \\ ${ }^{7}$ Institut für Halbleiter-und-Festkörperphysik, Johannes Kepler University, A-4040 Linz, Austria \\ ${ }^{8}$ Commissariat à l'Energie Atomique, INAC/SP2M-UJF, F-38054 Grenoble, France \\ ${ }^{9}$ Institute of Materials Science, University of Tsukuba, Tsukuba, Ibaraki 305-8573, Japan \\ ${ }^{10}$ Department of Electrical Engineering and Information Systems, The University of Tokyo, Tokyo 113-8656, \\ Japan \\ ${ }^{11}$ Department of Physical Electronics, Tokyo Institute of Technology, Tokyo 152-8552, Japan
}

(Dated: December 23, 2015)

This review presents the recent progress in computational materials design, experimental realization, and control methods of spinodal nanodecomposition under three- and two-dimensional crystal-growth conditions in spintronic materials, such as magnetically doped semiconductors. The computational description of nanodecomposition, performed by combining first-principles calculations with kinetic Monte Carlo simulations, is discussed together with extensive electron microscopy, synchrotron radiation, scanning probe, and ion beam methods that have been employed to visualize binodal and spinodal nanodecomposition (chemical phase separation) as well as nanoprecipitation (crystallographic phase separation) in a range of semiconductor compounds with a concentration of transition metal (TM) impurities beyond the solubility limit. The role of growth conditions, codoping by shallow impurities, kinetic barriers, and surface reactions in controlling the aggregation of magnetic cations is highlighted. According to theoretical simulations and experimental results the TM-rich regions appear in the form of either nanodots (the dairiseki phase) or nanocolumns (the konbu phase) buried in the host semiconductor. Particular attention is paid to Mn-doped group III arsenides and antimonides, TM-doped group III nitrides, Mn- and Fe-doped Ge, and Cr-doped group II chalcogenides, in which ferromagnetic features persisting up to above room temperature correlate with the presence of nanodecomposition and account for the application-relevant magnetooptical and magnetotransport properties of these compounds. Finally, it is pointed out that spinodal nanodecomposition can be viewed as a new class of bottom-up approach to nanofabrication.

\section{Contents}

I. Introduction

II. Theory of spinodal nanodecomposition

A. Ab initio materials design

B. Pairing energy

C. Monte Carlo simulation of nanodecomposition

1. Dairiseki phase

2. Konbu phase

D. Superparamagnetic blocking phenomena

E. Mixing energy; nucleation and spinodal regions

F. Cahn-Hilliard theory of spinodal decomposition

III. Spinodal nanodecomposition in (Ga,Mn)As

A. Fabrication methods and nanocomposite structure

B. Magnetic properties

C. Magneto-optical phenomena

D. Magnetoresistance

1. Films

2. Spin-valve structures

*Electronic address: ksato@mat.eng.osaka-u.ac.jp

${ }^{\dagger}$ Electronic address: alberta.bonanni@jku.at
E. Spinodal nanodecomposition in other magnetic III-V compounds

IV. Spinodal nanodecomposition in $(\mathrm{Ga}, \mathrm{Mn}) \mathrm{N}$

A. A controversial system

B. Experimental evidences for spinodal nanodecomposition in $(\mathrm{Ga}, \mathrm{Mn}) \mathrm{N}$

C. Co-doping with shallow impurities and $\delta$-doping in $(\mathrm{Ga}, \mathrm{Mn}) \mathrm{N}$

V. Spinodal nanodecomposition in $(\mathrm{Ga}, \mathrm{Fe}) \mathbf{N}$

A. Diluted $(\mathrm{Ga}, \mathrm{Fe}) \mathrm{N}$ : current status

B. Fabrication and properties of spinodally decomposed $(\mathrm{Ga}, \mathrm{Fe}) \mathrm{N}$

C. Fe distribution from nanoscale characterization

D. Phase diagram of the spinodal decomposition in $(\mathrm{Ga}, \mathrm{Fe}) \mathrm{N}$

E. Co-doping with shallow impurities in $(\mathrm{Ga}, \mathrm{Fe}) \mathrm{N}$

F. Magnetic properties of $(\mathrm{Ga}, \mathrm{Fe}) \mathrm{N}$

I. Spinodal nanodecomposition in (Ge,Mn

A. Mn dilution in germanium

B. From Mn dilution to phase separation

1. Review of experimental results

C. Growth and structure of thin $(\mathrm{Ge}, \mathrm{Mn})$ films with spinodal decomposition

1. Sample preparation

2. Morphology of $(\mathrm{Ge}, \mathrm{Mn})$ films 
3. Lateral and vertical control of nanocolumns

4. Two-dimensional spinodal decomposition

5. Crystal structure of nanocolumns

D. Magnetic properties

1. Crystalline (Ge,Mn) nanocolumns / Ge(001)

2. Crystalline $(\mathrm{Ge}, \mathrm{Mn})$ nanocolumns with partial relaxation / $\mathrm{Ge}(001)$

3. Amorphous (Ge,Mn) nanocolumns / Ge(001)

4. $\mathrm{Ge}_{3} \mathrm{Mn}_{5}$ clusters / Ge(001)

VII. Spinodal nanodecomposition in $(\mathrm{Ge}, \mathrm{Fe})$

A. Introduction

B. MBE growth

C. Structural and chemical characterization

D. Magneto-optical characterization

VIII. Spinodal nanodecomposition in $(\mathrm{Zn}, \mathrm{Cr}) \mathrm{Te}$

A. Introduction

B. Chemical phase separation and its control by the Fermi level position

1. Visualization of $\mathrm{Cr}$ distribution

2. Superparamagnetic behavior due to the $\mathrm{Cr}$ aggregation

3. Mechanism of $\mathrm{Cr}$ aggregation

4. Magnetooptical and magnetotransport properties 50

C. Crystallographic phase separation and konbu phase

1. Structural nanocharacterization

2. Magnetic properties

D. Summary

IX. Prospects of spinodal nanotechnology

X. Summary and outlook

List of abbreviations

Acknowledgments

References

\section{INTRODUCTION}

The detection of ferromagnetic features persisting up to above room temperature in a variety of magnetically doped semiconductors and oxides has been one of the most surprising developments in materials science over recent years (Bonanni, 2007; Coey et al., 2010; Dietl, 2003 Fukumura and Kawasaki, 2013; Kobayashi et al. 2008, Kuzemsky, 2013; Liu et al., 2005; Makarova, 2010, Nealon et al., 2012; Pearton et al., 2003, Roever et al. 2011, Sawicki et al. , 2013, Yao et al. | 2012). In particular, the presence of robust ferromagnetism in technologyrelevant semiconductors (e.g., GaN and $\mathrm{Si}$ ) and oxides (e.g., $\mathrm{ZnO}$ and $\mathrm{TiO}_{2}$ ) has promised to open the door to a wide exploitation in devices of remarkable spintronic functionalities found in dilute ferromagnetic semiconductors [such as (Ga,Mn)As] below their Curie temperature $T_{\mathrm{C}}$, so far not exceeding $200 \mathrm{~K}$ (Dietl and Ohno, 2014, Jungwirth et al., 2014; Sato et al. 2010). However, it is increasingly clear that high- $T_{\mathrm{C}}$ nonmetal ferromagnets represent a distinct class of systems in which the strength of ferromagnetic (FM) features correlates neither with the density of band holes necessary to mediate long-range interactions between diluted spins nor with an average concentration of magnetic ions.

In this review we focus on the latest progress in the investigations of structural and magnetic properties of dilute magnetic semiconductors (DMSs), such as group III pnictides, e.g., GaAs and GaN, group II chalcogenides, e.g., ZnTe, and group IV elemental semiconductors, e.g., $\mathrm{Ge}$, doped with transition metal (TM) ions, either $\mathrm{Cr}$, $\mathrm{Mn}$, or Fe. These studies have pointed out that, except for $\mathrm{Mn}$ in II-VI compounds (Giebultowicz et al., 1993, Pajączkowska, 1978), the fundamental premise of DMSs on the random distribution of magnetic ions over cation sites is often not valid, and that the aggregation of magnetic cations is strictly correlated with the presence of high- $T_{\mathrm{C}}$ ferromagnetism in these systems. As described here, this striking conclusion has been drawn from stateof-the-art theoretical modeling and experimental investigations. More specifically, the present understanding of semiconductors doped with transition metals has been built by combining:

- Ab initio computational studies providing the magnitude of chemical interactions between magnetic cations depending on their distance, charge state, and location (surface versus bulk); the interaction energies constitute the input parameters for kinetic simulations of spinodal nanodecomposition within the Monte Carlo method or by solving the relevant Cahn-Hilliard equation.

- Extensive epitaxy and post-growth processing protocols, including codoping and annealing, allowing to establish the relationship between fabrication conditions and physical properties.

- Comprehensive nanocharacterization investigations employing ever improving methods of electron microscopy, synchrotron radiation, ion beams, and scanning probes that provide elementspecific information on atom distributions, charge states, magnetic properties, and defects with spatial resolution down to the nm range.

- Scrupulous magnetization and magnetic resonance studies involving also the extensive examination of reference samples containing nominally no magnetic ions but otherwise grown and processed in the same way as the films under investigation.

According to the results reviewed in this paper, this methodology has made it possible to bring to light theoretically and experimentally a number of unanticipated properties of magnetically doped semiconductors. The new findings can be summarized as follows:

1. In DMSs, open orbitals of TM impurities not only provide localized spins but also, via $p-d$ hybridization, contribute to the bonding, which usually results in attractive forces between magnetic cations and in a miscibility gap in the thermodynamic phase diagram of the alloy. 
2. The actual TM distribution, for given fabrication conditions, post-growth processing, and epitaxial strain, is determined by a competition between attractive forces, entropy terms, and kinetic barriers at the growth surface or in the sample volume. This results in the coexitance of TM ions in random cation-substitutional positions and in regions with high concentrations of magnetic constituents, either commensurate with the host lattice (chemical phase separation) or in the form of precipitates (crystallographic phase separation). The process of TM aggregation is referred to as spinodal nanodecomposition although nucleation mechanisms (binodal decomposition) are involved in many cases.

3. The buried regions with high concentrations of magnetic constituents [called condensed magnetic semiconductors (CMSs), even if an insulator-tometal transition occurred locally] appear as TMrich nanodots (distributed randomly over the sample volume or clustered in defined planes) or in the form of TM-rich nanocolumns extending along specific crystal directions.

4. The incorporation and distribution of magnetic ions depend on codoping by shallow impurities or electrically active defects, which rather than changing the carrier concentration alter the valence of the TM ions and, thus, the chemical forces and kinetic barriers controlling the aggregation of magnetic cations either at the growth surface or in the film volume.

5. Because of the bonding to host atoms and the effects of strains, the structural and magnetic properties of particular CMS nanocrystals (NCs) may not yet be listed in chemical compendia and have to be assessed experimentally.

6. Depending on the predominant character of the exchange interactions, the spins within the individual CMS NCs exhibit FM, ferrimagnetic (FR), or antiferromagnetic (AF) spin ordering that owing to the high concentrations of TM ions persists typically to above room temperature (RT). At the same time, local strains, the character of interfaces, internal spin-orbit interactions, and the shapes of TM-rich regions control the magnetic anisotropy of particular CMS NCs.

7. Samples of nominally the same DMS with a given average TM concentration, can show diverse structural and macroscopic magnetic properties, as the relative abundance of randomly distributed TM ions and NCs formed by various phase separation processes depends on fabrication conditions, codoping, and defect content. Except for DMSs with a large concentration of holes, randomly distributed TM spins can order only at low temperatures, whereas the NCs can lead a FM, AF, and/or superparamagnetic behavior persisting up to high temperatures.

8. As known, noninteracting ferromagnetic nanoparticles exhibit a superparamagnetic behavior. However, decomposed magnetic alloys show typically temperature-independent narrow and leaning magnetic hysteresis loops up to the ordering temperature of the relevant CMS, even if the diameters of the TM-rich regions are in the sub $10 \mathrm{~nm}$ range. These superferromagnetic characteristics point to the important role of dipole interactions or strain-mediated spin-dependent coupling between the NCs. Ferromagnetic like features can result also from uncompensated spins at the surface of AF NCs.

9. The outstanding microscopic structure of decomposed alloys points to novel functionalities beyond those foreseen for hole-mediated uniform dilute FM semiconductors. Nevertheless, similar to the case of uniform systems, the macroscopic magnetotransport and magnetooptical properties of decomposed magnetic alloys are usually correlated with macroscopic magnetic characteristics. However, decomposed alloys additionally show a mixing between diagonal and nondiagonal components of the conductivity tensors and the persistence of magnetooptical effects in the spectral region below the band gap.

10. Magnetic studies of thin epitaxial layers containing a small quantity of magnetic impurities are challenged by the uncontrolled contamination of samples by FM nanoparticles and microparticles.

Altogether, one can now obtain various semiconductors with spatial distributions of TM cations on demand. In particular, by selecting appropriate growth conditions, layers' layout, codoping, and/or processing protocols it becomes possible to fabricate either a uniform DMS or a decomposed alloy with a predefined (i) character of the phase separation (chemical versus crystallographic), (ii) type of TM aggregation (nanodots versus nanocolumns), (iii) plane at which nanodots assemble, and (iv) chemical and magnetic properties of the precipitates. This command over the structural and magnetic properties offers a spectrum of opportunities for the design and realization of modulated systems with properties and functionalities encountered neither in uniform DMSs nor in the case of self-assembled quantum dots or nanowires of nonmagnetic semiconductors.

As seen in the Table of Contents, the main body of the present review consists of three major parts.

First, we discuss theoretical aspects of nanodecomposition in magnetically doped semiconductors (Sec.II). We start (Sec.II.A by presenting first-principles $a b$ initio approaches and their applications (Sec.II.B) for determining the chemical forces between magnetic ions, either in bulk or at the growth surface, as well as their dependence 
on codoping with shallow impurities. In Secs.II.C.1 and II.C.2 we describe Monte Carlo simulations of threedimensional (3D) and two-dimensional (2D) spinodal decomposition, corresponding respectively to the spontaneous formation of TM-rich nanodots in the film volume and to the self-assembly of TM-rich nanocolumns during the epitaxy. Taking into account shape magnetic anisotropy, samples containing nanodots and nanocolums exhibit quite different superparamagnetic properties, as discussed in Sec. II.D. Next, in Sec.II.E the computed phase diagrams of some binary alloy are presented, allowing us to recall the notions of miscibility gap, nucleation, and spinodal decomposition. We also emphasize the importance of kinetic barriers in the process of TM aggregation. These concepts make it possible to discuss, in Sec.II.F, the Cahn-Hilliard equation quantifying the dynamics of spinodal decomposition. As a whole, theoretical modeling elucidates the nature of nanodecomposition for different combinations of hosts, TM ions, shallow impurities, and fabrication procedures.

Second, in Secs.III VIII experimental studies of nanodecomposition in specific families of magnetically doped semiconductors are presented. Whenever possible the findings are discussed in the context of the theoretical predictions outlined in Sec.II. We start (Sec.III) with the model FM semiconductor (Ga,Mn)As. For this system pioneering works aiming at the fabrication of GaAs containing MnAs nanoprecipitates were carried out (De Boeck et al., 1996; Shi et al., 1996). Furthermore, early $a b$ initio studies of (Ga,Mn)As and related systems revealed the presence of strong attractive forces between TM cation pairs in DMSs (van Schilfgaarde and Mryasov, 2001). We describe the chemical and crystallographic phase separations occurring in (Ga,Mn)As under annealing or epitaxy at appropriately high temperatures. Structural information on Mn-rich (Mn,Ga)As NCs is linked to FM-like features as well as to device-relevant magnetic circular dichroism (MCD) and magnetotransport characteristics of this nonocomposite system. The understanding of ferromagnetism in decomposed (Ga,Mn)As allows us to assess the origin of high- $T_{\mathrm{C}}$ in $(\mathrm{Ga}, \mathrm{Mn}) \mathrm{P}$, (In,Mn)As, (Ga,Mn)Sb, and (In,Mn)Sb.

In Secs. IV and V we describe investigations of group III nitrides, doped with either $\mathrm{Mn}$ or $\mathrm{Fe}$ and, in some studies, codoped with $\mathrm{Si}$ or $\mathrm{Mg}$. In the case of $(\mathrm{Ga}, \mathrm{Mn}) \mathrm{N}$ (Sec.IV) a rich collection of magnetic properties at similar average Mn content is observed. The nanodecomposition scenario is confirmed by structural nanocharacterization demonstrating the correlation of high- $T_{\mathrm{C}}$ and low- $T_{\mathrm{C}}$ with respectively the presence and absence of a phase separation. Similar experiments point to spinodal decomposition, in the form of nanocolumns, in $(\mathrm{Al}, \mathrm{Cr}) \mathrm{N}$ ( $\mathrm{Gu}$ et al. , 2005). Section $\mathrm{V}$ is devoted to $(\mathrm{Ga}, \mathrm{Fe}) \mathrm{N}$, the subject of particularly comprehensive structural and magnetic studies. The collected data provide evidences for spinodal decomposition as well as for the precipitation of various Fe-rich $(\mathrm{Fe}, \mathrm{Ga})_{x} \mathrm{~N}$ NCs whose composition $x$ (and thus magnetic properties), abundance, and location in predefine planes can be controlled by growth conditions, codoping by $\mathrm{Si}$ and $\mathrm{Mg}$, and architecture.

Sections VI and VII present the outcome of nanodecomposition studies on Mn- and Fe-doped Ge, respectively, in which the TM distribution is uniform or either chemical or crystallographic phase separation is observed depending on the growth temperature. In addition to detailed structural and magnetic investigations, comprehensive MCD and magnetotransport data are available for these systems. Particularly relevant are works on the formation of Mn-rich nanocolumns along the [001] growth direction in (Ge,Mn) deposited onto Ge(001) substrates.

As described in Sec.VIII in the case of (Zn,Cr)Te spinodal decomposition results in either Cr-rich nanodots or nanocolumns assuming a $\langle 111\rangle$ orientation, even for epitaxy on (001) substrates. Despite chemical heterogeneity there is a strict correlation between magnetization, MCD, and anomalous Hall effect (AHE) in these alloys. Furthermore, nanodecomposition and, thus, other associated properties are strongly affected by changing the concentrations of donors or acceptors, either by manipulating with stoichiometry via altering the intensities of the molecular beams or by codoping with I or N, respectively. Pioneering work drawing attention to the possibility of spinodal nanodecomposition in (Zn,Cr)Se (Karczewski et al. 2003) is also discussed.

In the third part of our review, Sec.IX, we discuss application prospects of the remarkable properties revealed for decomposed magnetically doped semiconductors over the recent years. We emphasize the possibility of bottom-up nanotechnology based on the control of spinodal nanodecomposition ("spinodal nanotechnology"). It is pointed out that decomposed magnetic alloys consisting, for instance, of a semiconductor with embedded NCs of a FM metal can exhibit functionalities that cannot be realized employing either uniform FM metal films or semiconductor quantum dot layers. Finally, in Sec.X, we summarize the main conclusions stemming from studies of heterogeneous DMSs and present an outlook on open questions and challenges ahead. Furthermore, a list of abbreviations is provided.

As seen, the physics of high- $T_{\mathrm{C}}$ ferromagnetism in magnetically doped oxides (Coey et al., 2010, Fukumura and Kawasaki, 2013, Li et al., 2012; Sawicki et al., 2013), carbon derivatives (Kuzemsky, 2013, Makarova, 2010, Wang et al., 2014), and some other systems (Nealon et al. 2012, Roever et al., 2011; Rylkov et al., 2012, Yao et al., 2012) is beyond the scope of this review. In particular, the question of ferromagnetism originating from spins residing on defects (Coey et al., 2010, Zhou, 2014) or on open $p$ shells (Volnianska and Boguslawski, 2010), or mediated by defects or by residual impurities like hydrogen (Li et al. 2012 ) is not addressed here. We note that not only several authors have considered theoretically whether ferromagnetism would be possible without magnetic elements (Bouzerar and Ziman, 2006, Droghetti et al. , 2009, Du et al. | 2012, Elfimov et al. | 2002; Ivanovskii, 2007; Kenmochi et al., 2004a, 2005, 2004b; Mavropoulos et al., 
2009, Volnianska and Boguslawski, 2010) but also the role of spinodal nanodecomposition was examined in this context (Seike et al., 2012, 2013a b).

As emphasized in our survey, the recent progress in understanding high- $T_{\mathrm{C}}$ DMSs results, to a large extent, from the application of various powerful nanocharacterization tools. Nevertheless, we do not discuss in detail relevant experimental techniques, as many of them were recently reviewed in a collection (Bonanni, 2011) that contains also useful information about the methodology of magnetic measurements on thin films employing superconducting quantum interference device (SQUID) magnetometry (Sawicki et al. , 2011). A related experimental challenge is the contamination by FM nanoparticles and microparticles that already reside in the substrate or can be incorporated during the growth, annealing, etching, handling, or storing of particular samples. Some instructive examples were disclosed (Abraham et al. 2005 , Grace et al., 2009; Makarova et al., 2006, Matsubayashi et al. (2002) but presumably much more cases, even if spotted, have not been published.

\section{THEORY OF SPINODAL NANODECOMPOSITION}

\section{A. Ab initio materials design}

Ideally theoretical studies should predict the microscopic TM distribution, including the presence of chemical and crystallographic phase separations, and the corresponding electronic and magnetic properties at given growth conditions and codoping by shallow impurities. As already reviewed elsewhere (Sato et al., 2010$)$, firstprinciples methods for electronic structure calculations have played the important role in predicting various properties of homogeneous and heterogenous DMSs without referring to any experimental parameters. This is mainly due to the success of the local density approximation (LDA) in the density functional theory (DFT) (Dreizler and Gross, 1995, Hohenberg and Kohn, 1964; Kohn and Sham, 1965).

In the DFT, a many electron system is described by using the electron density $n(\mathbf{r})$, i.e., it is proven that the many electron wave function $\Phi$ and the expectation value of a physical quantity $\langle A\rangle$ can be written as a functional of $n(\mathbf{r})$, such as $\Phi[n(\mathbf{r})]$ and $A[n(\mathbf{r})]$, respectively. In particular, one can search for the ground state by minimizing the total energy functional $E[n(\mathbf{r})]$ with respect to $n(\mathbf{r})$. In the Kohn-Sham scheme, the total energy functional is formulated by referring to a one-electron system and given as,

$$
\begin{array}{r}
E[n(\mathbf{r})]=T_{\mathrm{op}}[n(\mathbf{r})]+\int n(\mathbf{r}) v(\mathbf{r}) d^{3} r+ \\
+\frac{1}{2} \iint e^{2} \frac{n(\mathbf{r}) \cdot n\left(\mathbf{r}^{\prime}\right)}{\left|\mathbf{r}-\mathbf{r}^{\prime}\right|} d^{3} r d^{3} r^{\prime}+E_{\mathrm{xc}}[n(\mathbf{r})] .
\end{array}
$$

Here $T_{\text {op }}[n(\mathbf{r})]$ is the electrons' kinetic energy, the second term is the energy of the electrons in an external poten- tial $v(\mathbf{r})$, the third term is the classical Hartree interaction energy, and the last term is the exchange-correlation energy. For the exchange-correlation energy, a rigorous expression is not known and an efficient approximation is needed (Dreizler and Gross, 1995).

The standard approximation is the LDA within which $E_{\mathrm{xc}}$ is taken as the exchange-correlation energy of homogeneous electron gas (Ceperly and Alder, 1980, Dreizler and Gross, 1995, Perdew and Wang, 1986). Thus, within the LDA, $E_{\mathrm{xc}}$ is calculated as a function of the local electron density, i.e., $E_{\mathrm{xc}}[n(\mathbf{r})] \rightarrow E_{\mathrm{xc}}(n(\mathbf{r}))$. This approximation sounds inaccurate since the electron density distribution in a real material varies strongly depending on the position r. Furthermore, the computations are carried out for supercells containing typically less than 100 atoms and assuming periodic boundary conditions. Actually, despite these approximations, the LDA, or its spin-polarized version, the local spin density approximation (LSDA), is accurate enough for explaining many physical properties of various materials (Martin, 2004). Recently, LDA, LSDA, and their modifications are used not only for understanding experimental results but also for designing new functional materials.

It is well known that the phase diagrams of a number of alloys exhibit a solubility gap in a certain concentration range. Particularly low is the solubility of TM impurities in semiconductors. An exception here is a large solubility of $\mathrm{Mn}$ in II-VI compounds, in which Mn atoms remain distributed randomly over the substitutional cation sites up to concentrations often exceeding 50\% (Furdyna and Kossut, 1988; Pajączkowska, 1978). The large solubility of $\mathrm{Mn}$ in II-VI compounds can be assigned to the truly divalent character of Mn whose $d$ states little perturb the $s p^{3}$ tetrahedral $s p$ bonds as both the lower $d^{5}$ (donor) and the upper $d^{6}$ (acceptor) Hubbard levels are respectively well below and above the band edges (Dietl, 1981, 2002; Zunger, 1986).

We describe now two ab initio approaches that have provided quantitative information on the thermodynamic stability of particular DMS alloys $\mathrm{A}_{1-x} \mathrm{TM}_{x} \mathrm{~B}$ as well as input parameters [such as the pairing energy $E_{d}$ and mixing energy $\Delta E(x)$ ] for studies of decomposition dynamics by the Monte Carlo simulations and by the CahnHilliard equation, respectively. Within the first method one evaluates a change of the system energy associated with bringing two or more TM ions to neighboring cation positions. If this pairing energy $E_{d}$ is negative, i.e., there is an attractive chemical force between TM ions, their distribution may not be random. The second approach involves the evaluation of the alloy energy in comparison to weighted energies of the end compounds. A positive value of this mixing energy $\Delta E(x)$ points to the instability of the alloy. 


\section{B. Pairing energy}

The pairing energy $E_{d}$ (known also as heat of reaction or pair interaction energy) for a DMS alloy (A,TM)B is evaluated from total energies $E$ corresponding to three different contents of TM cations in the supercell van Schilfgaarde and Mryasov, 2001),

$$
\begin{aligned}
E_{d} & =E\left[\left(\mathrm{~A}_{N-2}, \mathrm{TM}_{2}\right) \mathrm{B}_{N}\right]+E\left[\mathrm{~A}_{N} \mathrm{~B}_{N}\right]- \\
& -2 E\left[\left(\mathrm{~A}_{N-1}, \mathrm{TM}\right) \mathrm{B}_{N}\right],
\end{aligned}
$$

where $N$ is the total number of cations in the supercell. The magnitudes of $E_{d}$ for TMs at the nearest-neighbor cation positions were determined employing various DFT implementations for (Ga,Mn)As (Birowska et al., 2012 , Mahadevan et al., 2005, Sato et al. , 2005; van Schilfgaarde and Mryasov, 2001); (Ga,V)As, (Ga,Cr)As, and (Ga,Fe)As (Mahadevan et al., 2005); (Ga,Mn)N (Bogusławski and Bernholc, 2006; Chan et al., 2008; Das et al., 2003; Gonzalez Szwacki et al., 2011; Raebiger et al. 2014 Sato et al., 2005, van Schilfgaarde and Mryasov, 2001); (Ga,Cr)N (Cui et al., 2005, Gonzalez Szwacki et al., 2011, Mahadevan et al., 2005, van Schilfgaarde and Mryasov, 2001); (Ga,Fe)N (Gonzalez Szwacki et al., 2011; Navarro-Quezada et al. , 2011); (Ge,Mn,Cr,Co) (Continenza et al. , 2006a); (Zn,Mn)Te (Kuroda et al. 2007$)$, and (Zn,Cr) Te (Da Silva et al., 2008; Fukushima et al. 2006b; Kuroda et al., 2007; Raebiger et al., 2014).

In the case of the nearest-neighbor Mn cations in ZnTe, $E_{d}=21 \mathrm{meV}$ (Kuroda et al. , 2007), the result consistent with a large solubility of $\mathrm{Mn}$ in II-VI compounds, as mentioned previously. In contrast, $E_{d}=-160 \mathrm{meV}$ for Cr cations in $\mathrm{Zn}_{0.95} \mathrm{Cr}_{0.05} \mathrm{Te}$ (Fukushima et al. $2006 \mathrm{~b}$ ), which indicates that $\mathrm{Cr}$ cations should aggregate provided that diffusion barriers can be overcome at the growth or annealing temperature. Similarly, the pairing energy of Mn cation dimers was computed to be $E_{d}=-120 \mathrm{meV}$ in GaAs and $-300 \mathrm{meV}$ in GaN van Schilfgaarde and Mryasov, 2001). Being determined by $p-d$ hybridization, the magnitude of $\left|E_{d}\right|$ decays quickly with the distance between TM cations, as shown in Fig. 1.

A number of experimentally relevant extensions of this approach has been proposed.

Beyond dimers: The formations of larger TM cation clusters than dimers were considered and their magnetic properties assessed (Cui et al. , 2007, Das et al. 2003 , Gonzalez Szwacki et al. , 2011; Hynninen et al., 2006b, Mahadevan et al. 2005; Navarro-Quezada et al., 2011, van Schilfgaarde and Mryasov, 2001). In this case (van Schilfgaarde and Mryasov, 2001),

$$
\begin{array}{r}
E_{d}^{(n)}=E\left[\left(\mathrm{~A}_{N-n}, \mathrm{TM}_{n}\right) \mathrm{B}_{N}\right]+E\left[\mathrm{~A}_{N} \mathrm{~B}_{N}\right]- \\
-E\left[\left(\mathrm{~A}_{N-n+1}, \mathrm{TM}\right) \mathrm{B}_{N}\right]-E\left[\left(\mathrm{~A}_{N-1}, \mathrm{TM}\right) \mathrm{B}_{N}\right] .
\end{array}
$$

This represents the energy change associated with the trapping of one more TM cation by a cluster consisting of $n-1$ TM cations. Early studies (van Schilfgaarde and Mryasov, 2001) suggested that $E_{d}^{(n)} \simeq 0$ for $n=3$ in the case of $(\mathrm{Ga}, \mathrm{Mn}) \mathrm{As},(\mathrm{Ga}, \mathrm{Mn}) \mathrm{N}$, and $(\mathrm{Ga}, \mathrm{Cr}) \mathrm{N}$, i.e., that decomposition of these alloys into systems containing clusters with three TM cations is favored energetically. This conclusion is not supported by a more recent investigation indicating that $E_{d}^{(n)}$ remains strongly negative up to at least $n=4$ (Gonzalez Szwacki et al. 2011), as shown in Fig.2. This work also pointed out that coupling between pairs of $\mathrm{Mn}$ and of $\mathrm{Cr}$ ions is FM in wurtzite (wz) GaN, whereas the interaction between Fe ions is AF. These expectations are discussed in comparison to experimental results in Secs. IV and V.

The structure of Mn clusters in (Ga,Mn) N and the interactions of the magnetic $\mathrm{Mn}$ ions and clusters have been studied through first-principles calculations and $T_{\mathrm{C}}$ was evaluated using mean-field and Monte Carlo methods (Hynninen et al., 2007). It was found that joining substitutional Mn ions to clusters is energetically favorable and especially the structures of two to four Mn ions formed around a single $\mathrm{N}$ ion are most stable. These clusters are always found to have a FM ground state, and FM intercluster interactions are also present even at relatively long distances. For randomly distributed Mn clusters, high $T_{\mathrm{C}}$ s are obtained at high Mn concentrations (Hynninen et al., 2007 ). The calculated $T_{\mathrm{C}}$ is expected
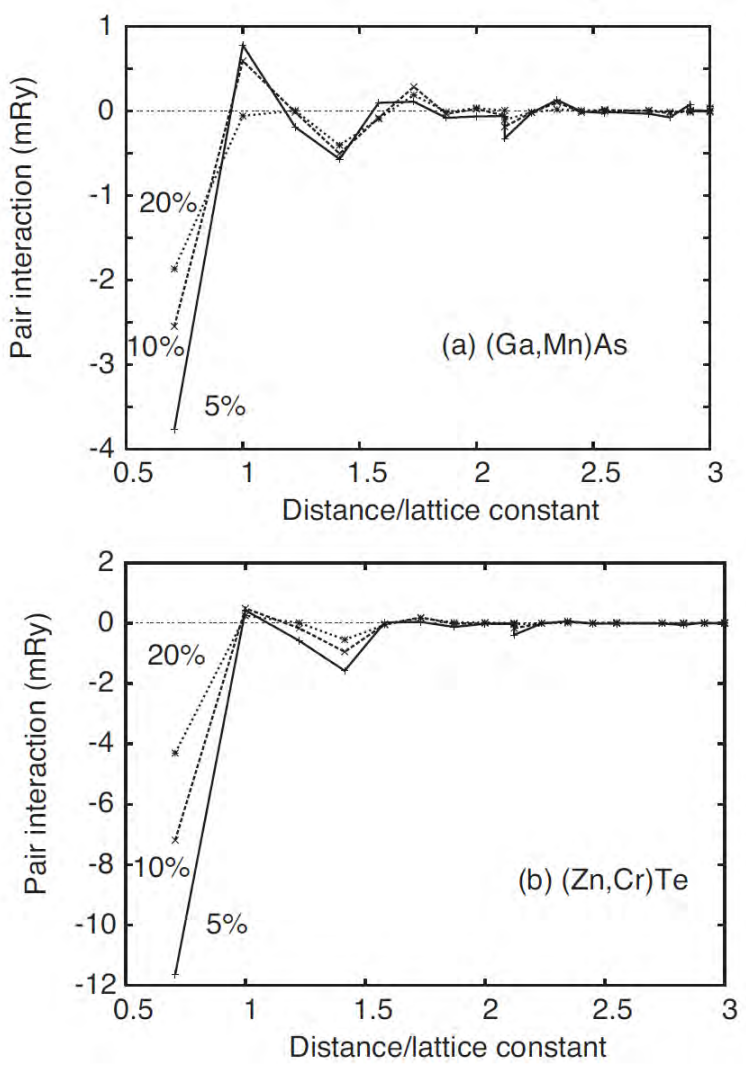

FIG. 1 Calculated pair interaction energies $E_{d}$ as a function of the distance between TM pairs in (a) $\mathrm{Ga}_{1-x} \mathrm{Mn}_{x}$ As (from Sato et al. 2005) and (b) $\mathrm{Zn}_{1-x} \mathrm{Cr}_{x} \mathrm{Te}$ (from Fukushima et al. $2006 \mathrm{~b})$ with various average concentrations $x$ of TM ions. 


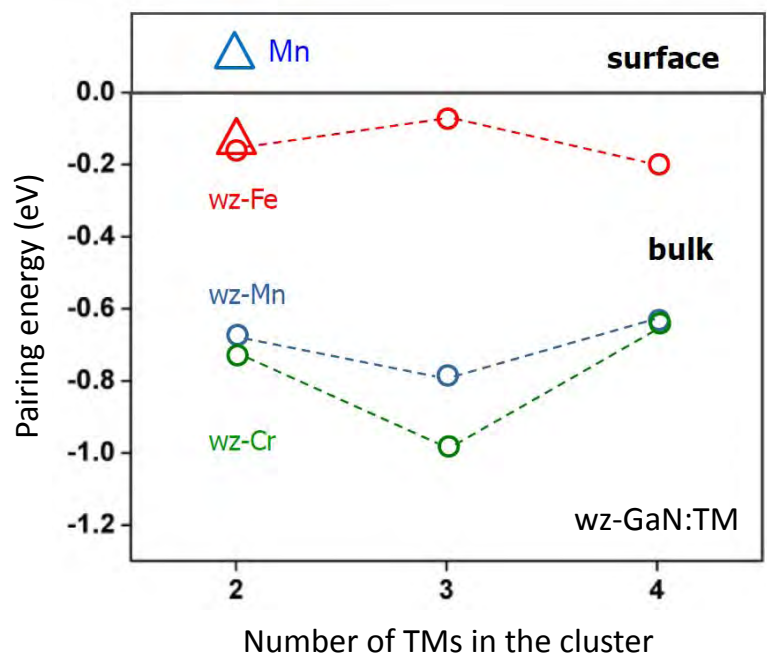

FIG. 2 (Color online) Pairing energies $E_{d}^{(n)}$ as a function of the number $n$ of TM cations in $\mathrm{Cr}, \mathrm{Mn}$, and Fe clusters in bulk wz-GaN (circles) and on $\mathrm{Ga}(0001)$ surface (triangles). Adapted from Gonzalez Szwacki et al., 2011.

to depend dramatically on the microscopic cluster distribution (Hynninen et al. $2006 \mathrm{a})$, giving a possible explanation for the remarkable spread in experimental $T_{\mathrm{C}}$ values for $(\mathrm{Ga}, \mathrm{Mn}) \mathrm{N}$ (see Sec.IV.A). The complexity of the Mn-clustering has been highlighted by further DFT calculations where the coexistence of AF and FM coupling has been found for various configurations, different charge states and spatial distribution of the clusters (Cui et al. 2007).

Nanocolumns and nanodots: It was noted that in the presence of attractive forces between TM cations, the layer-by-layer epitaxy can result in the growth of TMrich nanocolumns (Fukushima et al., 2006b). The resulting structure was named the "konbu phase", where konbu means seaweed in Japanese. The presence of the konbu phase was experimentally demonstrated in the case of $(\mathrm{Al}, \mathrm{Cr}) \mathrm{N}$ ( $\mathrm{Gu}$ et al., 2005$),(\mathrm{Ge}, \mathrm{Mn})$ (Sec.VI), and $(\mathrm{Zn}, \mathrm{Cr}) \mathrm{Te}$ (Sec.VIII). Often, however, the TM-rich regions appear in the form of nanodots, the case of, annealed (Ga,Mn)As (see Sec.III). Since the cross section looks then like a marble (dairiseki in Japanese), the decomposed state is referred to as the "dairiseki phase" (Sato et al. 2005). Results of Monte-Carlo simulations designed to show the formation of either konbu or dairiseki phases are presented in Secs.II.C.1 and II.C.2.

Surface aggregation: Since the initial step of the TM aggregation is expected to occur on the epitaxy plane, pairing energies of TM impurities residing on surfaces relevant to epitaxial processes were evaluated (Birowska et al., 2012, Gonzalez Szwacki et al., 2011; NavarroQuezada et al. . 2011). As presented in Fig.2, $E_{d}$ values for $\mathrm{Mn}$ and $\mathrm{Cr}$ cation dimers on the (0001) wz-GaN surface become positive $\left(E_{\mathrm{d}} \simeq 170 \mathrm{meV}\right.$ and $280 \mathrm{meV}$, respectively), whereas $E_{d}$ remains negative for Fe pairs $\left(E_{\mathrm{d}}=-120 \mathrm{meV}\right)$, favoring the formation of Fe-rich clusters during the epitaxy (Gonzalez Szwacki et al., 2011). This explains a much lower solubility limit of Fe comparing to $\mathrm{Mn}$ in epitaxial films of wz-GaN but does not elucidate why it is difficult to grow epitaxially $(\mathrm{Ga}, \mathrm{Cr}) \mathrm{N}$ with a large concentration of randomly distributed $\mathrm{Cr}$ ions (see, Secs. IV] and V]. Furthermore, a question addressed was how the spatial distribution of TM ions in epitaxial films could be affected by nonequivalence of certain crystal directions on the surface. It was found for (Ga,Mn)As deposited onto an unreconstructed surface of (001)GaAs that $E_{d}$ is by $1 \mathrm{eV}$ smaller for Mn pairs residing along the [110] axis compared to the [110] case (Birowska et al. , 2012). Puzzling uniaxial anisotropies found in $(\mathrm{Ga}, \mathrm{Mn})$ As were explained by the lowering of crystal symmetry associated with the nonrandom distribution of $\mathrm{Mn}$ dimer orientations, setting in during the epitaxy (Birowska et al., 2012).

Role of codoping: It was suggested that codoping of DMSs with shallow donors or acceptors constitutes an efficient method of controlling the TM aggregation (Dietl, 2006). This way of affecting the process of nanodecomposition operates if there are band-gap states derived from TM $d$ orbitals that can trap carriers supplied by shallow impurities (for a compilation of TM-related levels in various hosts see, e.g., Dietl and Ohno, 2003). The corresponding change in the charge state and valency of the magnetic ions modifies chemical and spindependent interactions between TM impurities. As an example, the energy of the screened Coulomb repulsion between two elementary charges residing on the nearestneighbor cation sites in the GaAs lattice is $280 \mathrm{meV}$. Accordingly, a surplus of charge on TM ions brought by codoping with shallow dopants or by electrically active defects can outweigh the gain of energy stemming from $p-d$ hybridization and impede the $\mathrm{NC}$ assembling (Bogusławski and Bernholc, 2006, Dietl, 2006, Ye and Freeman, 2006). This intuitive picture was checked by ab initio computations for $(\mathrm{Ga}, \mathrm{Mn}) \mathrm{N}$ (Bogusławski and Bernholc, 2006), (Ga,Fe)N (Navarro-Quezada et al., 2011), (Zn,Cr) Te (Da Silva et al., 2008; Kuroda et al., 2007), and $(\mathrm{Ga}, \mathrm{Cr})$ As (Da Silva et al. 2008$)$. As shown in Fig. 3, the value of $E_{\mathrm{d}}$ attains a minimum in ZnTe when the two $\mathrm{Cr}$ cations are in the $2+$ charge state (Da Silva et al. 2008). However, the computation results shown in the same plot also indicate that in GaAs $E_{\mathrm{d}}$ goes through a minimum for the $\mathrm{Cr}^{2+}$ case, rather than for the $\mathrm{Cr}^{3+}$ pairs, as might be expected for III-V compounds. Experimental verifications of these ideas have been found for $(\mathrm{Ga}, \mathrm{Fe}) \mathrm{N}$ codoped with $\mathrm{Si}(\mathrm{Sec} . \mathrm{V})$ and for $(\mathrm{Zn}, \mathrm{Cr}) \mathrm{Te}$ codoped with I and $\mathrm{N}$ or deposited under growth conditions allowing to control the concentration of electrically active point defects (Sec.VIII). The effect of codoping is also discussed theoretically in Sec.II.E exploiting the concept of the mixing energy.

Impurity and defect complexes: Electrically active impurities and defects not only alter the position of the 


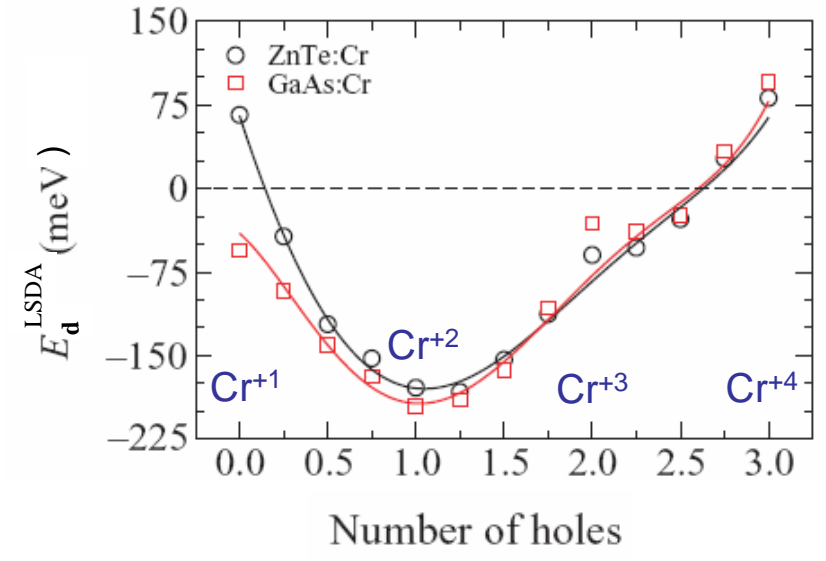

FIG. 3 Computed energy change resulting from bringing two Cr impurities to the nearest-neighbor cation positions in $\mathrm{ZnTe}$ and GaAs depending on the charge state, i.e., the number of holes in the $\operatorname{Cr} d^{5}$ shell. Adapted from Da Silva et al. 2008.

Fermi level and, thus, the TM charge and spin state but can form with TM ions complexes characterized by specific structural and magnetic properties. Prompted by experimental results summarized in Secs. IV]and $\mathrm{V}$, magnitudes of $E_{d}^{(n, k)}$, where $k$ denotes the number of shallow dopants in the cluster were evaluated for various complexes in wz-GaN, including $\mathrm{Mn}-\mathrm{Mg}_{k}$ (Devillers et al. 2012) as well as $\mathrm{Fe}_{n}-\mathrm{Mg}_{k}$ and $\mathrm{Fe}_{n}-\mathrm{Si}_{k}$ in the bulk and at the surface (Navarro-Quezada et al., 2011). It was also established theoretically that Mn-D cation dimers, where $\mathrm{D}$ denotes either $\mathrm{P}, \mathrm{As}$, or $\mathrm{Sb}$ donor, allow to increase the incorporation of $\mathrm{Mn}$ in substitutional positions in both Si and Ge (Zhu et al. 2008).

Crystallographic phase separation: The electronic structure and magnetic properties of antiperovskite $\mathrm{GaMn}_{3} \mathrm{~N}$ - frequently identified as a precipitated phase in $(\mathrm{Ga}, \mathrm{Mn}) \mathrm{N}$ beyond the solubility limit of $\mathrm{Mn}$ into GaN (see Sec.IV.B) - have been studied by means of full potential linear muffin tin orbital (FP-LMTO) methods and compared to the results for cubic $\mathrm{Mn}_{4} \mathrm{~N}$ and $\mathrm{Mn}_{3} \mathrm{~N}$. The total energy of these phases in different magnetic states, respectively FM, FR, and AF is reported in Fig.4.

\section{Monte Carlo simulation of nanodecomposition}

From pairing energy considerations given in Sec.II.B, nanodecomposition is expected to occur in a number of DMSs. In order to predict the resulting TM distribution, it is relevant to simulate crystal growth. For this purpose, a hybrid method, combining ab initio and Monte Carlo simulations, was developed (Sato et al., 2005).

Within this method, the alloy is described by an Ising model with the Hamiltonian of the system written as,

$$
H=\frac{1}{2} \sum_{i \neq j} V_{i j} \sigma_{i} \sigma_{j}
$$

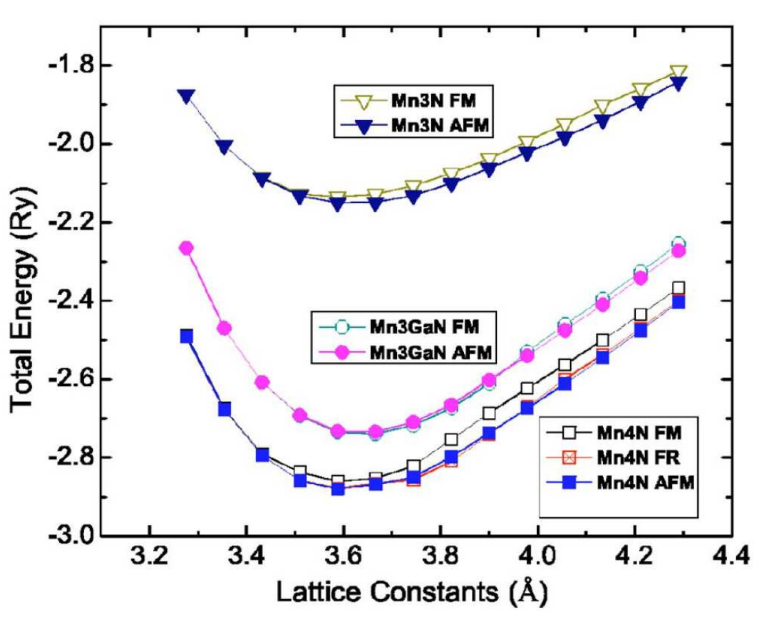

FIG. 4 (Color online) Total energy for $\mathrm{Mn}_{4} \mathrm{~N}, \mathrm{GaMn}_{3} \mathrm{~N}$, and $\mathrm{Mn}_{3} \mathrm{~N}$ in different magnetic ordering configurations. From Miao et al. 2005.

where $V_{i j}$ is the effective pair interaction energy between two TM cations at sites $i$ and $j, \sigma_{i}$ is the occupation index of the site $i$ by the TM ion, i.e., $\sigma_{i}=1$ if site $i$ is occupied by a TM atom while $\sigma_{i}=0$ if site $i$ is occupied by a host atom. The effective interaction is calculated as $V_{i j}=V_{A A}+V_{B B}-2 V_{A B}$ for a two component alloy $\mathrm{AB}$, where $V_{A A}, V_{B B}$ and $V_{A B}$ are the potential energies for the sites $i j$ occupied by $\mathrm{AA}, \mathrm{BB}$, and $\mathrm{AB}$ atoms, respectively. The effective pair interactions $V_{i j}$ are evaluated for the medium obtained within the coherent potential approximation (CPA) by using the generalized perturbation method proposed by Ducastelle and Gautier, 1976 in the formulation of Turchi et al., 1988.

The calculated effective pair interactions in $(\mathrm{Ga}, \mathrm{Mn}) \mathrm{As}$ and $(\mathrm{Zn}, \mathrm{Cr})$ Te have been plotted in Fig. 1 as a function of the pair distance. By definition of $V_{i j}$, a negative $V_{i j}$ indicates effective attractive interactions between the same kind of atoms and repulsive interactions between different kind of atoms. As shown in Fig.1. pair interactions between magnetic impurities are effectively attractive.

Once the pair interactions in the Ising model are obtained, one can perform the Monte Carlo simulation of the Ising model to obtain the distribution of TM cations in a given semiconductor host at nonzero temperature. The Kawasaki dynamics is used to relax the system (Landau and Binder, 2000). First, a large supercell [typically $14 \times 14 \times 14$ face-centered cubic (fcc) conventional cubic cell] is considered and the magnetic cations are randomly distributed in the cell. Then, one picks up one host-TM pair and their position is exchanged. When the change in the energy due to the exchange is negative, this process is allowed. When the energy is positive, it is decided whether this process is allowed or not by using the Metropolis criterion. This Monte Carlo step is repeated many times until the system reaches the thermal equilibrium. In the present study, a nonequilibrium state, namely the system is quenched within a very short time 
interval which is not sufficient for the complete relaxation of the impurity distribution and then frozen. This situation is simulated by interrupting the iteration after a certain number of Monte Carlo steps (Sato et al., 2005). This procedure assumes that $V_{i j}$ is the only relevant energy scale and, in particular, that there are no kinetic barriers for cation exchange and diffusion over the lattice sites. The role of such barriers is discussed in Secs.II.E and I.F.

\section{Dairiseki phase}

Figure 5 visualizes decomposition in $(\mathrm{Ga}, \mathrm{Mn}) \mathrm{N}$, as revealed by Monte Carlo simulations. In the studied example, the average concentration of $\mathrm{Mn}$ in the simulation cell is $5 \%$ or $20 \%$, and 100 Monte Carlo steps per Mn site are performed at temperature $k_{B} T_{\mathrm{b}} / V_{01}=0.5$, where $V_{01}$ is the chemical pair interaction energy between the nearest neighbors. Because of the chemical pair interactions, the $\mathrm{Mn}$ atoms attract each other, which results in the formation of clusters. In each cluster, the Mn atoms occupy nearest-neighbor sites in order to decrease the energy as much as possible. As seen in Fig. 5(b), the Mn concentration in the cluster is almost $100 \%$ and the shape of the cluster is nearly spherical. If the Mn average concentration is low, the clusters are separated. For higher concentrations, the average size of the clusters becomes larger and at $20 \%$ the clusters are connected and percolate through the cell [Fig. 5 (d)]. Since the cross section of the decomposed system looks like a marble, the decomposed state of magnetically doped semiconductors was named the "dairiseki phase" (Sato et al., 2005), where dairiseki means marble in Japanese.

As shown in the previous studies on ferromagnetism in DMSs with short-range spin-spin interactions, at low concentrations $T_{\mathrm{C}}$ is suppressed (Sato et al. , 2010, Stefanowicz et al. , 2013). For example, below the percolation threshold [20\% for the fcc structure (Stauffer and Aharony, 1994)] $T_{\mathrm{C}}$ is 0 for systems with interactions only between the nearest neighbors. As shown in Fig.5(d), owing to decomposition there are many percolating paths already at $20 \%$ and the decomposition considerably affects the magnetic properties of the system. In order to see the effects of decomposition, $T_{\mathrm{C}}$ values were calculated as a function of Monte Carlo steps (Fukushima et $a$ a. , 2006a). In order to include in the $T_{\mathrm{C}}$ calculations the distribution of magnetic impurities, the random phase approximation (RPA) proposed by Bouzerar et al., 2005 and Hilbert and Nolting, 2004 was employed (Fukushima et al., 2006a). It is known that this method correctly reproduces the magnetic percolation effects and predicts reasonable $T_{\mathrm{C}}$ magnitudes, close to the exact Monte Carlo values. Figure 6 shows the calculated $T_{\mathrm{C}}$ of (Zn,Cr)Te within the RPA as a function of Monte Carlo steps.

As shown in Fig.6, at low concentrations the decomposition works to lower $T_{\mathrm{C}}$ values. Due to decomposition

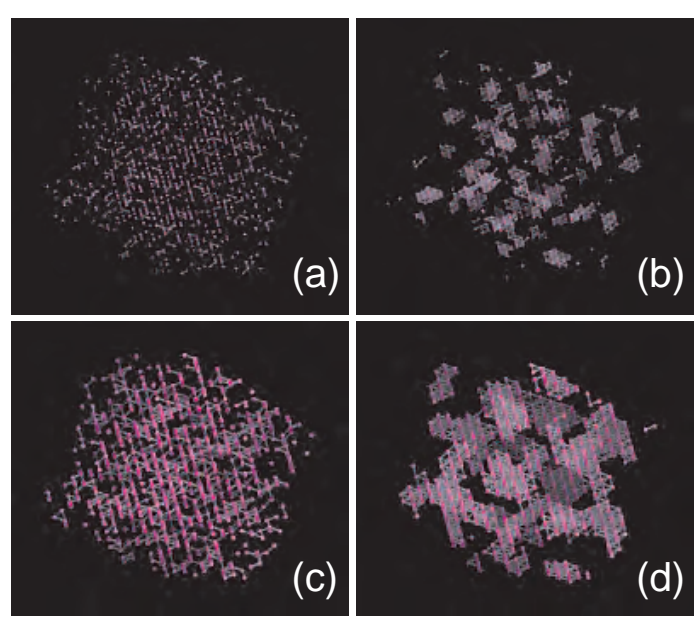

FIG. 5 (Color online) Mn configuration in (Ga,Mn)N. (a) and (c) refer to completely random configurations, (b) and (d) after 100 Monte Carlo steps (decomposed phase). Mn concentrations are $5 \%$ for (a) and (b), and $20 \%$ for (c) and (d). Nearest neighbor MnMn pairs are connected by bars. From Sato et al. 2005.

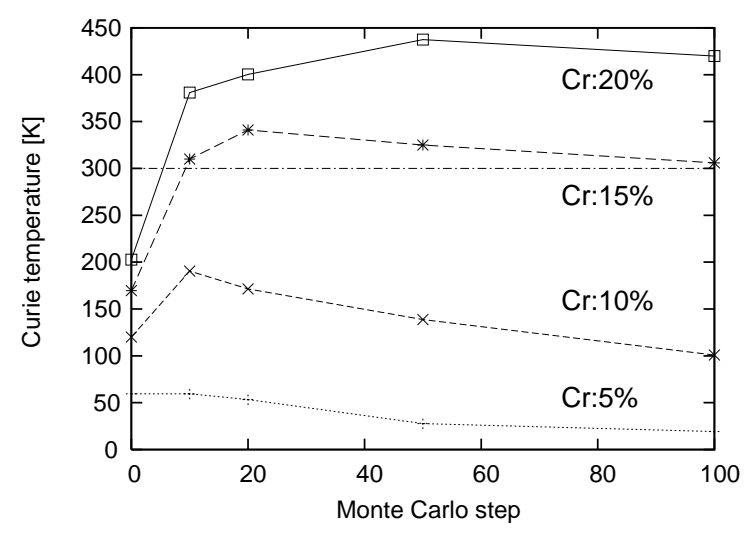

FIG. 6 Calculated Curie temperature of $(\mathrm{Zn}, \mathrm{Cr}) \mathrm{Te}$ as a function of Monte Carlo steps in the simulation of decomposition. Curie temperatures are calculated by using the random phase approximation. From Fukushima et al. 2006a.

many spatially isolated clusters are formed, as shown in Fig.5(b). Since the exchange interactions between TM atoms are short-range in this case, there is strong FM coupling between TM moments in the cluster, but FM correlations between clusters are weak. This is why $T_{\mathrm{C}}$ of the whole system is suppressed by decomposition. In the case of $(\mathrm{Zn}, \mathrm{Cr}) \mathrm{Te}$ after $100 \mathrm{MC}$ steps, nonzero values of $T_{\mathrm{C}}$ are predicted, and the system is FM. However when the exchange interactions are more short range, the system becomes superparamagnetic after the decomposition. For higher concentrations, because of the increase in the number of the nearest-neighbor pairs, $T_{\mathrm{C}}$ rises as the decomposition proceeds. The increase of $T_{\mathrm{C}}$ is ob- 
served already at $15 \%$, which is below the percolation threshold; thus it is found that the decomposition effectively lowers the magnetic percolation threshold.

\section{Konbu phase}

In addition to growth temperature and TM concentration, one can control the dimensionality of the growth process. In the simulations presented in Sec.II.C.1, the magnetic impurities can diffuse in any direction, therefore TM aggregation occurs in three dimensions. In actual experiments, molecular beam epitaxy (MBE) or metalorganic vapor phase epitaxy (MOVPE) are standard methods for crystal growth. On a surface with low coverage, the migration of atoms on the surface occurs relatively freely, but it is difficult to diffuse into the layers beneath, i.e., the diffusion is limited only to the uppermost layer. Thus under such crystal-growth conditions, the dimensionality of the decomposition is automatically controlled. Since the decomposition occurs layer by layer and the exchange of atoms between layers is prohibited under this condition, one can expect an anisotropic shape for the clusters formed by the decomposition. On the first layer, small clusters are formed due to the decomposition, and then the impurity atoms deposited on the second layer gather around the nuclei in the first layer. This process is repeated many times, and finally clusters with elongated shape along the crystal-growth direction are produced (Fukushima et al. 2006b).

In Fig.7 results of Monte Carlo simulations are shown for the layer-by-layer growth of $(\mathrm{Zn}, \mathrm{Cr}) \mathrm{Te}$ with the average $\mathrm{Cr}$ concentration of $5 \%$. The growth direction is from the bottom to the top of the cube. As expected, the Cr cations form clusters with columnar shape. This state was named the konbu phase (Fukushima et al. $2006 \mathrm{~b}$ ), where konbu means seaweed in Japanese. Apparently the size of the clusters is much larger than the clusters found in the dairiseki phase. In the konbu phase, the clusters are spatially well separated. The $T_{\mathrm{C}}$ value of each cluster is expected as high as the one of CrTe in a zinc-blende (zb) structure, presumably close to RT. The $T_{\mathrm{C}}$ magnitude of the konbu phase shown in Fig.7(a) by using the RPA method is only $15 \mathrm{~K}$. This low value is due to the absence of magnetic interactions between separated columnar structures. However, once the magnetic percolation paths are introduced between the structures, $T_{\mathrm{C}}$ should increase considerably. To visualize this effect, delta doping of $\mathrm{Cr}$ for the first and the last layers was assumed in the simulations [Fig. $7(\mathrm{~b})$ ], leading to $T_{\mathrm{C}}$ of $346 \mathrm{~K}$ (Fukushima et al. 2006b). In reality, magnetization blocking phenomena brought about by magnetic anisotropy (see Sec.II.D and long-range dipole interactions between clusters' magnetizations can lead to a large magnitude of apparent $T_{\mathrm{C}}$.

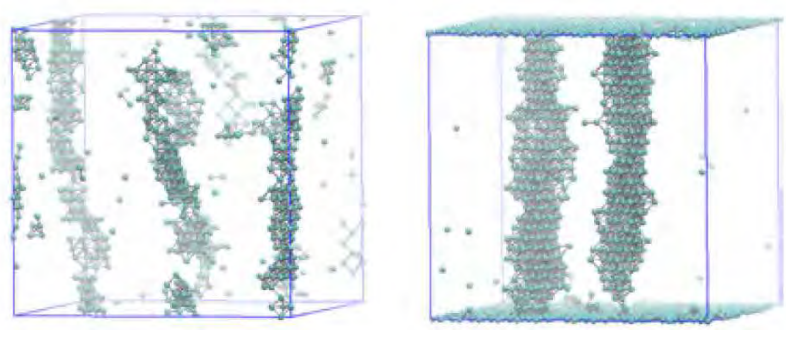

FIG. 7 (Color online) Monte Carlo simulations of phase separation in $(\mathrm{Zn}, \mathrm{Cr}) \mathrm{Te}$ under the layer-by-layer crystal growth condition (konbu phase). Average concentration of $\mathrm{Cr}$ is $5 \%$ for both (a) and (b), except for the first and the last layer in (b). In (b) delta-doping is simulated by depositing $80 \%$ of $\mathrm{Cr}$ on the first and the last layers. Cr positions in the crystal are indicated by small spheres and they are connected by bars if they occupy the nearest-neighbor sites. Adapted from Fukushima et al. 2006b

\section{Superparamagnetic blocking phenomena}

If we focus only on a thermodynamic quantity such as a $T_{\mathrm{C}}$ value, there is not much difference between the dairiseki phase and the konbu phase for low average TM concentrations. For both phases as a result of decomposition, $T_{\mathrm{C}}$ goes to 0 and the system becomes superparamagnetic, if the interactions are short-range. On the other hand, if we look into the magnetization process, the dairiseki phase and the konbu phase behave very differently due to superparamagnetic blocking phenomena associated with shape anisotropy.

To reverse the magnetization direction of nanomagnets within a single magnetic domain state, magnetic anisotropy energy should be overcome. For a system with uniaxial magnetic anisotropy, the magnetic anisotropy energy is $K V \sin ^{2} \theta$, where $K, V$ and $\theta$ are the anisotropy energy density, the volume of the system, and the angle between the magnetization direction and the magnetic easy axis, respectively. In general, shape and crystalline anisotropies contribute to the magnitude of $K$. The existence of the energy barrier between the easy directions results in a finite relaxation rate of the magnetization direction $1 / \tau \propto \exp \left(-K V / k_{B} T\right)$. Accordingly, for larger systems (but small enough to keep single domain nature), the relaxation time $\tau$ becomes longer and the magnetization direction is effectively fixed along the initial direction at low temperatures. This is the so-called superparamagnetic blocking phenomenon (Aharoni, 1996).

In the present cases of decomposed systems, the columnar structure contains high concentration of magnetic impurities. Because of the FM exchange interactions between magnetic impurities, the self-assembled structure with a high TM concentration behaves as a nanomagnet. The radius of the columnar structure is a few nanometers and within this length scale, we can expect that the structure contains a single magnetic domain.

As shown in Secs.II.C.1 and II.C.2, it is possible to 

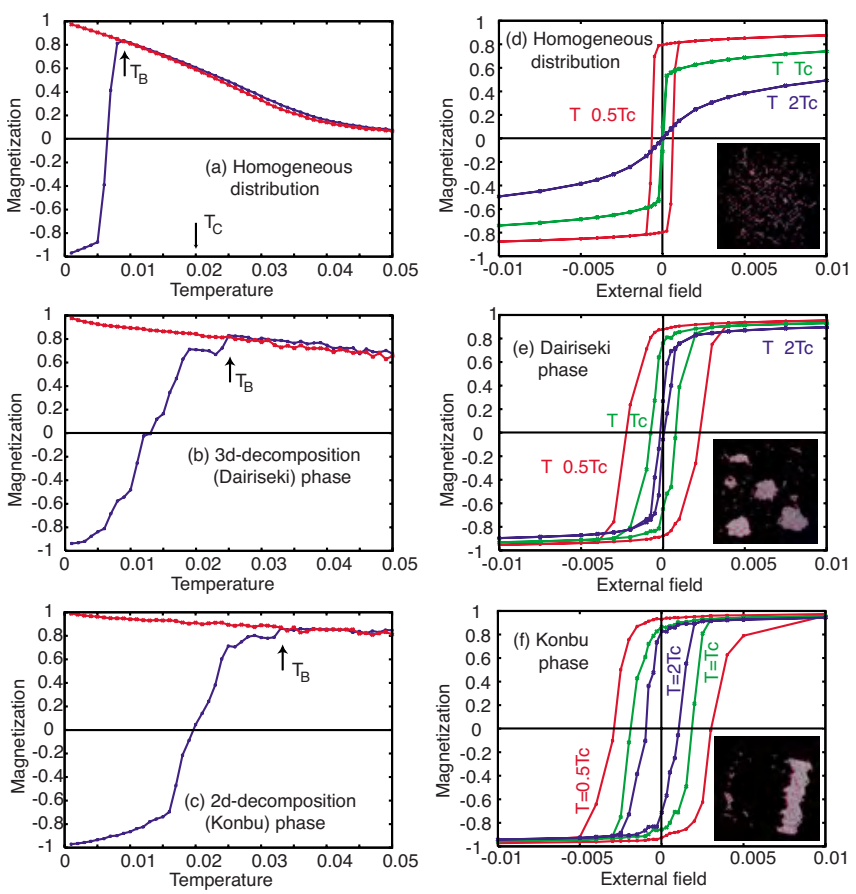

FIG. 8 (Color online) Superparamagnetic blocking phenomenon in $(\mathrm{Ga}, \mathrm{Mn}) \mathrm{N}$ according to Monte Carlo simulations. Left-hand panels: Magnetization as function of temperature starting from parallel or antiparallel configurations of initial magnetization to the external field. Right-hand panels: Magnetization as a function of an external field. The simulations were performed for the uniform Mn distribution (a) and (d), the dairiseki phase (b) and (e), and the konbu phase (c) and (f). The insets in the lower panels show snapshots of the Mn distribution in $(\mathrm{Ga}, \mathrm{Mn}) \mathrm{N}$ for the respective phases. Temperature $\left(k_{B} T\right)$ and the external magnetic field are scaled by the strength of the nearest-neighbor exchange energy $J_{01}$. From Sato et al. 2007.

control the volume of the clusters formed by decomposition and, hence, sample magnetization via the superparamagnetic blocking phenomenon. In order to demonstrate this idea, the magnetization process was simulated by the Monte Carlo technique (Katayama-Yoshida et al. 2007b) employing the method elaborated by Dimitrov and Wysin, 1996. In Fig.8 the calculated temperature and field dependence of magnetization are shown taking the shape part of magnetic anisotropy into account. The simulations were performed for a uniform TM distribution, the dairiseki phase, and the konbu phase of $(\mathrm{Ga}, \mathrm{Mn}) \mathrm{N}$. As shown in the magnetization curves, for the system with larger clusters, the cohesive field becomes greater and hysteresis loop opens wider, i.e., one obtains a harder magnet. Furthermore, magnetization as a function of temperature was calculate for increasing temperature starting from anti-parallel and parallel configurations of magnetization with respect to the external magnetic field. Above the blocking temperature $T_{\mathrm{b}}$, the direction of the magnetization flips and the two lines coincide. It is demonstrated in Figs. 8(a-c) that $T_{\mathrm{b}}$ becomes higher for the dairiseki and konbu phases.

Until now many experimental reports on the magnetism of TM-doped semiconductors have been published, but often they are not consistent, particularly for wide band-gap hosts. As shown in Sec.II.B. energies of chemical pair interactions between magnetic impurities are rather large, especially in wide band-gap DMSs, so that these systems have a strong tendency toward decomposition. Since typically epitaxy is a nonequilibrium process, its outcome, particularly the degree of decomposition, depends sensitively on growth and processing conditions. As a result, the size, shape, and content of TM-rich NCs resulting from decomposition vary strongly from experiment to experiment. These considerations explain, at least partly, inconsistency in magnetic properties reported by various groups for the same material.

\section{E. Mixing energy; nucleation and spinodal regions}

In order to determine the temperature and TM concentration range corresponding to nanodecomposition, a lattice model, originally developed by Flory and Huggins for the polymer-mixing problem (Flory, 1942; Huggins, 1941, Rubinstein and Colby, 2003), was adapted for DMSs (Chan et al., 2008; Hai et al., 2011; Sato et al., 2007). Within this approach, the free energy $F(x)=\Delta E(x)-T S(x)$ of a random alloy $\mathrm{A}_{1-x} \mathrm{TM}_{x} \mathrm{~B}$ is described by the mixing energy $\Delta E(x)$ and the entropy $S(x)$ per one cation of the form

$$
\begin{aligned}
\Delta E(x) & =E\left(\mathrm{~A}_{1-x} \mathrm{TM}_{x} \mathrm{~B}\right)-(1-x) E(\mathrm{AB}) \\
& -x E(\mathrm{TMB}) \\
S(x) & =-k_{B}[x \ln x+(1-x) \ln (1-x)] .
\end{aligned}
$$

If $\Delta E(x)>0$, the alloy is unstable against decomposition into the end compounds (Swalin, 1970) but at $T>0$ a tendency to randomization described by the entropy term becomes important. Figure 9 shows schematically the behavior of $F(x)$ for a convex function $\Delta E(x)>0$ and $T>0$. It is seen that for such a case $F(x)$ there are two points $B_{1}$ and $B_{2}$ at compositions $x_{1}$ and $x_{2}$, between which $F(x)>F\left(x_{1}\right)+F\left(x_{2}\right)$. Hence, for $x_{1}<x<x_{2}$ the thermal equilibrium state corresponds to the binodal decomposition, i.e., in the case of DMSs, to the nucleation of TM-rich NCs of content $x_{2}$ in the TM-poor matrix of the concentration $x_{1}$. The region $x_{1}<x<x_{2}$ corresponds to the miscibility gap at given temperature $T$.

Often, however, the alloy in some range between $x_{1}$ and $x_{2}$ is stable against small fluctuations $\pm \Delta x$ of local composition. Since changes in the local free energy density integrated over the volume encompassing many fluctuations is proportional to $(\Delta x)^{2} \partial^{2} F / \partial x^{2}$, the alloy can remain in a metastable state as long as $\partial^{2} F / \partial x^{2}>0$, i.e., in the composition range, in which $F(x)$ is a concave function. In contrast, in the region between the points $S_{1}$ and $S_{2}$ in Fig. 9 , where $\partial^{2} F / \partial x^{2}<0$, there is no 


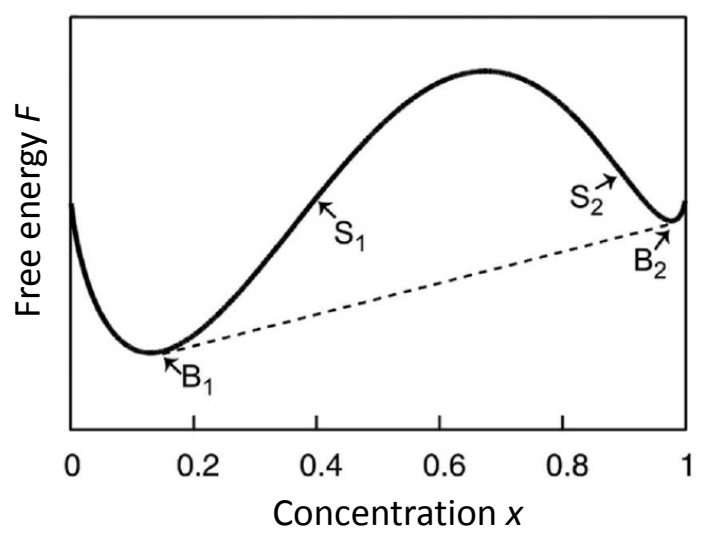

FIG. 9 Typical dependence of DMS free energy $F$ on TM concentration $x$. Binodal decomposition can occur between points $\mathrm{B}_{1}$ and $\mathrm{B}_{2}$, the ends of the common tangent segment of $F(x)$ (dashed line). Spinodal decomposition occurs between points $\mathrm{S}_{1}$ and $\mathrm{S}_{2}$ corresponding to $\partial^{2} F / \partial x^{2}=0$. From Hai et al. 2011.

thermodynamic barrier separating the homogenous and non-uniform alloy.

The resulting spontaneous up hill diffusion process, called spinodal decomposition, leads to a system that is structurally homogenous but shows a modulation of the TM concentration. This mechanism of chemical phase separation is suitable for fabricating a $\mathrm{NC}$ system with rather uniform NC size and inter-NC distance (Jones, 2002). An ordering in NC positions can be enhanced further on by strain, similar to the case of self-assembling quantum dots in semiconductors (Stangl et al. 2004). As discussed in Secs. III and VI, a periodic-like arrangement of TM-rich NCs has been found in Mn-doped GaAs and Mn-doped Ge, respectively.

The CPA approach (Sato et al. 2007 ) and a cluster expansion method (Chan et al., 2008) were employed to evaluate from first principles the energy $E\left(\mathrm{~A}_{1-x} \mathrm{TM}_{x} \mathrm{~B}\right)$ and, thus, the mixing energy $\Delta E(x)$ of DMSs containing randomly distributed substitutional TM cations.

The CPA is particularly suitable for electronic structure calculations in the case of substitutional alloys (Akai, 1989, Akai and Dederichs, 1993, Shiba, 1971), such as DMSs. It is most efficiently combined with the Korringa-Kohn-Rostoker (KKR) method for the band structure calculation, particularly employing the MACHIKANEYAMA package (Sato et al., 2010) developed by Akai (Akai, 2002). Within the CPA, a configuration average of the alloy electronic structure is calculated by using a mean-field like procedure. For the first step one considers a hypothetical atom that describes the averaged system. The crystal of hypothetical atoms constitutes an effective CPA medium. By using the multiple scattering theory the scattering path operator of the host and of the impurity atoms in the CPA medium is determined. The weighted average of these scattering path operators with respect to the concentration should be

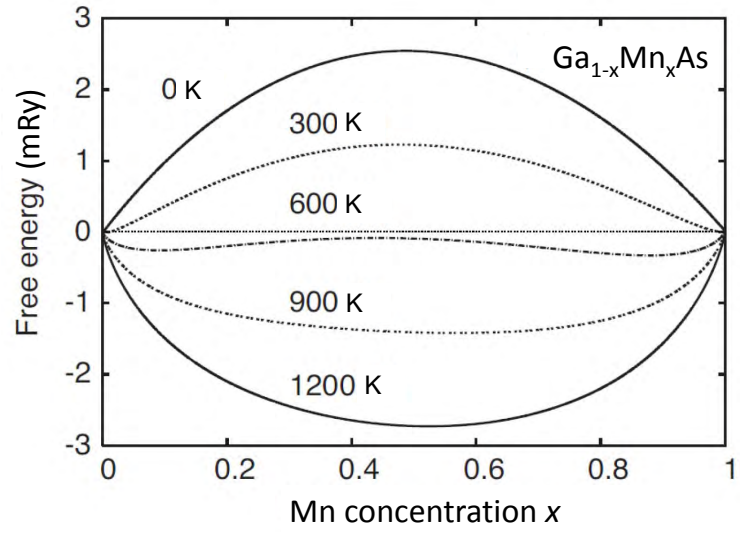

FIG. 10 Computed free energy $F(x)$ for $\mathrm{Ga}_{1-x} \mathrm{Mn}_{x}$ As at various temperatures. As shown schematically in Fig. 9 this plot allows one to determine composition ranges corresponding to binodal and spinodal decompositions at particular temperatures. From Sato et al. 2007.

equal to the scattering path operator of the hypothetical atom itself. This is the self-consistent equation to be solved by an iterative method for determining the CPA medium, providing electronic density of states and the total energy $E$ of the system. A more detailed explanation and practical formulation is provided by Gonis, 2000 .

The CPA method was employed to evaluate $\Delta E(x)$ for $\mathrm{Zn}_{1-x} \mathrm{Cr}_{x} \mathrm{Te}, \mathrm{Ga}_{1-x} \mathrm{Mn}_{x} \mathrm{As}, \mathrm{Ga}_{1-x} \mathrm{Cr}_{x} \mathrm{~N}$, and $\mathrm{Ga}_{1-x} \mathrm{Mn}_{x} \mathrm{~N}$ assuming zb structure and the experimental values of the lattice constants (Sato et al., 2007). The convex form of $\Delta E(x)$ demonstrated that spinodal decomposition can appear in these systems. A similar conclusion was derived from cluster expansion studies of zb-Ga $\mathrm{Ga}_{1-x} \mathrm{Mn}_{x} \mathrm{~N}$ (Chan et al., 2008$)$. Figure 10 shows, as an example, $F(x)$ for $\mathrm{Ga}_{1-x} \mathrm{Mn}_{x} \mathrm{As}$ at various temperatures (Sato et al. . 2007), which allows one to determine the spinodal line, i.e., the position of $S_{1}$ and $S_{2}$ points as a function of temperature. Such a spinodal line is presented in Fig. 11 for in zb-Ga $\mathrm{Ga}_{1-x} \mathrm{Mn}_{x} \mathrm{~N}$ (Chan et al. 2008). It is seen that the upper critical solution temperature, above which the alloy is miscible for any $x$, is as high as $3000 \mathrm{~K}$ in this case. Hence, within the temperature range relevant for epitaxial growth and post-growth annealing, the DMSs in question can undergo spinodal decomposition.

According to Figs.9 11, the range of compositions, at which alloy decomposition could appear, diminishes with increasing temperature. This is in qualitative disagreement with the long staying experimental observations which imply that lowering of epitaxy temperature is necessary for obtaining a uniform DMS (Munekata et al., 1989), whereas annealing at high temperatures allows to generate phase separation (De Boeck et al. 1 1996). This apparent contradiction, i.e., the existence of lower critical solution temperature, points to the importance of diffusion (kinetic) barriers precluding aggregation of TM 


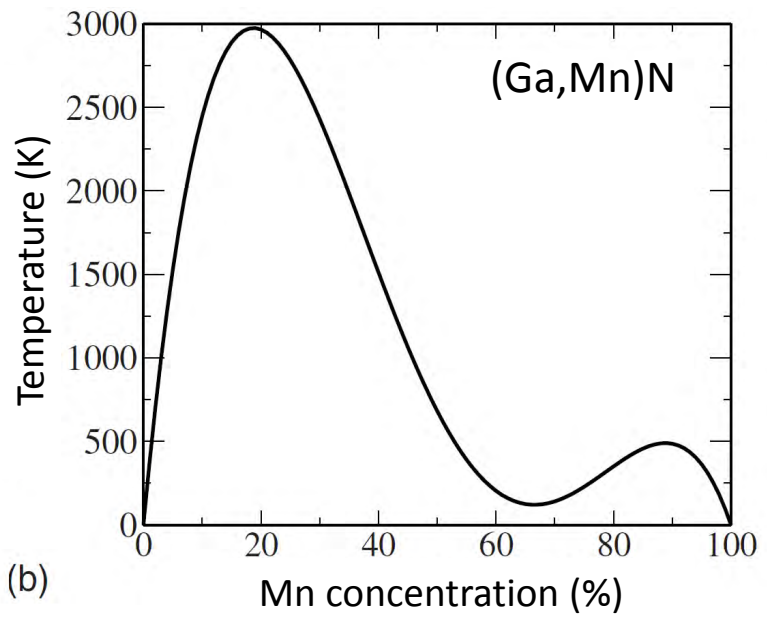

FIG. 11 Computed spinodal line for zinc-blende $\mathrm{Ga}_{1-x} \mathrm{Mn}_{x} \mathrm{~N}$. In the range of compositions $x$ between the lines at given temperature $T$ the alloy can undergo spinodal decomposition, so that at, e.g., $1000 \mathrm{~K} \mathrm{Ga}_{1-x} \mathrm{Mn}_{x} \mathrm{~N}$ decomposes into compounds with $x=0.03$ and 0.45. From Chan et al. 2008 .

cations if growth or annealing temperature is too low even if there is no thermodynamic barrier for spinodal decomposition. To our knowledge, in the case of DMSs, only the barrier height for diffusion of interstitial Mn and Li ions in GaAs has so far been theoretically evaluated (Bergqvist et al. 2011, Edmonds et al., 2004). At the same time, a phenomenological Cahn-Hillard theory of spinodal decomposition, presented in Sec.II.F was employed to describe properties of phase separation in DMSs (Hai et al. 2011). The influence of growth and processing conditions on nanodecomposition is discussed from the experimental perspective in Secs. III VIII.

Another important ingredient that has not been taken into account by the approaches leading to the results presented in Figs. 10 and 11 is the omission of interfacial energies. In particular, the comparison of free energies corresponding to the random alloy and the decomposed case referred to the thermodynamic limit, i.e., energies of TM ions at interfaces between TM-rich and TM-poor phases have been disregarded. In general, energy lowering associated with the TM aggregation is smaller for interfacial ions compared to the ions inside the cluster. This promotes the Oswald ripening, i.e., the formation of larger clusters at the expense of smaller ones. In extreme cases, small clusters, up to the so-call nucleation radius, are energetically unstable, which imposes a barrier for the nucleation process. Such a barrier can be lowered at crystal defects acting as nucleation centers or diffusion channels. According to the discussion of the pairing energies in Sec.II.B, even small clusters of TM cations are stable in a number of semiconductor compounds.

As already mentioned in Sec.II.B according to $a b$ initio studies, codoping of DMSs by electrically active impurities or defects affects considerably the TM pairing energies. This conclusion is supported by
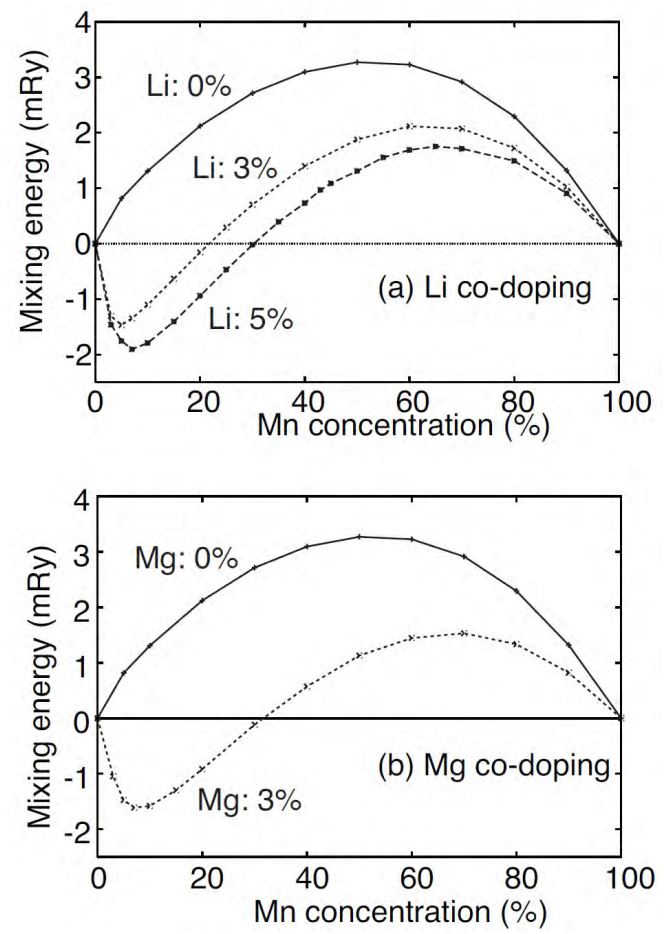

FIG. 12 Calculated mixing energy of (Ga,Mn)As as a function of Mn concentration with (a) interstitial Li codoping and (b) interstitial $\mathrm{Mg}$ codoping. From Bergqvist et al., 2011.

studies of the mixing energy $\Delta E(x)$ of $(\mathrm{Ga}, \mathrm{Mn}) \mathrm{As}$ (Bergqvist et al. 2011, Sato and Katayama-Yoshida, 2007), (Ga,Mn)N, (Ga,Mn)Cr, and (Zn,Cr)Te (Sato and Katayama-Yoshida, 2007) codoped with donors. In particular, the mixing energies of (Ga,Mn)As with interstitial impurities acting as donors, such as $\mathrm{Li}\left(\mathrm{Li}_{\text {int }}\right)$ and $\mathrm{Mg}$ $\left(\mathrm{Mg}_{\text {int }}\right)$, were computed (Bergqvist et al. 2011) As shown in Fig.12, the effect of codoping is dramatic and the the mixing energy is significantly modified. Particularly at low concentrations of $\mathrm{Mn}$, a negative and concave region is found in the calculated values of $\Delta E(x)$. This means that even at thermal equilibrium homogeneous doping of $\mathrm{Mn}$ is possible over a wide $x$ range. Similar effects were also found for other combinations of the host semiconductors and different substitutional donor impurities (Sato and Katayama-Yoshida, 2007).

The advantage of interstitial impurities (particularly $\mathrm{Li}_{\text {int }}$ ) over the substitutional ones is that interstitials can easily be removed by postgrowth annealing. To check the effectiveness of this process, Monte Carlo simulations of diffusion of interstitial impurities in $(\mathrm{Ga}, \mathrm{Mn}) \mathrm{As}$ were performed (Bergqvist et al., 2011). The results imply that since the binding energy between $\mathrm{Mn}_{\text {int }}$ and $\mathrm{Mn}_{\mathrm{Ga}}$ is much greater than in the case of $\mathrm{Li}_{\mathrm{int}}$, the lowtemperature annealing more effectively wipes away $\mathrm{Li}_{\text {int }}$ than $\mathrm{Mn}_{\text {int }}$ in $(\mathrm{Ga}, \mathrm{Mn}) \mathrm{As}$. 


\section{F. Cahn-Hilliard theory of spinodal decomposition}

One of the goal of decomposition theory is to describe spatial and temporal evolution of the local composition $x(\vec{r}, t)$ at a surface or in a volume of the alloy containing initially randomly distributed TM cations of the concentration $\bar{x}$. While it is rather difficult to determine $x(\vec{r}, t)$ in the nucleation regime, there exists a time-honored Cahn and Hilliard, 1958 theory of spinodal decomposition, discussed also in the context of phase separation in DMSs (Hai et al. , 2011). This approach exploits the Ginzburg-Landau theory of the continuous phase transitions, taking $u(\vec{r}, t)=x(\vec{r}, t)-\bar{x}$ as an order parameter. Furthermore, the interfacial effects are included to the lowest order in the gradient in the TM concentration $x(\vec{r})$. Under these assumptions the functional of the free energy assumes the form,

$$
F_{\text {tot }}[u(\vec{r})]=N_{0} \int d \vec{r} F[u(\vec{r})]+\frac{1}{2} \kappa|\nabla u(\vec{r})|^{2} .
$$

where $N_{0}$ is the cation density and $F(x)$, in addition to the contributions given in Eqs.5 and 6, can contain a term $k u^{2}(\vec{r}, t) / 2$ describing an increase of the system energy associated with strain that builds in a decomposed system if the lattice constant depends on $x$. Along with the mixing energy $\Delta E(x), \kappa$ and $k$ are parameters of the model, which in principle can be determined experimentally or by $a b$ initio methods.

The distribution $u(\vec{r})$ under conditions of thermal equilibrium is provided by the variational minimization of $F_{\text {tot }}[u(\vec{r})], \delta F_{\text {tot }}[u(\vec{r})] / \delta u(\vec{r})=0$ with the constrain, imposed by the mass conservation, that $u(\vec{r})$ integrated over the relevant crystal surface or volume vanishes. This procedure implies that (i) the decomposition occurs at temperatures below the critical temperature $T_{c}$, where $\left.\partial^{2} F(u, T) / \partial u^{2}\right)\left.\right|_{0}<0$; (ii) the compositions of TM-rich NCs and TM-poor matrix are determined from $\partial\left[F(u)-\left.u(\partial F / \partial u)\right|_{0}\right] / \partial u=0$ and (iii) the spatial width of the transition region between these two domains scales with $\kappa^{1 / 2}$.

However, from the experimental viewpoint particularly relevant is the evaluation of $u(\vec{r}, t)$ during the early stage of decomposition, i.e., far from thermal equilibrium. The starting point is the continuity equation,

$$
\partial N_{0} u(\vec{r}, t) / \partial t=-\nabla \cdot \vec{j}(\vec{r}, t),
$$

where the current of TM cations $\vec{j}(\vec{r}, t)$ is driven by the gradient in the chemical potentials $\mu$,

$$
\vec{j}(\vec{r}, t)=M \nabla \mu(\vec{r}, t) .
$$

Here, $M$ is the TM mobility, one more material parameter. Since the chemical potential corresponds to a change of the free energy by adding or removing one atom, its local nonequilibrium value is given by a variational derivative,

$$
\mu(\vec{r}, t)=-\delta F_{\text {tot }}[u(\vec{r}, t)] / \delta u(\vec{r}, t)
$$

with the constrain $\int d \vec{r} u(\vec{r}, t)=0$. This variational derivative and, thus, the chemical potential is not zero as $u(\vec{r}, t)$ does not yet correspond to the thermal equilibrium distribution. Inserting Eq.10 into Eq.9 and then Eq.9 into 8, one obtains the Cahn-Hilliard equation in the form

$$
\partial u(\vec{r}, t) / \partial t=M \nabla^{2} \partial\left[F(u)-\left.u(\partial F(u) / \partial u)\right|_{0}\right] / \partial u-\kappa \nabla^{2} u .
$$

There is a comprehensive literature devoted to mathematical aspects of this differential equation (NovickCohen, 2008). It is convenient to write its solution $u(\vec{r}, t)$ as a Fourier transform of a function $A(\vec{q}, t)$. If only the two lowest order terms are retained in the Taylor expansion of $F(u)$, a justified approximation at the early stage of decomposition, then

$$
A(\vec{q}, t)=A_{0}(\vec{q}) \exp [R(\vec{q}) t]
$$

where the aggregation rate

$$
R(q)=-M\left[\partial^{2} F(u) /\left.\partial u^{2}\right|_{0}+\kappa q^{2}\right] q^{2} .
$$

This formula shows that $R$ is positive, at least for sufficiently small $q$ values, in the spinodal decomposition range $\partial^{2} F(u) /\left.\partial u^{2}\right|_{0}<0$. In such a case, $R$ is governed by the cation mobility $M$ which, because of diffusion barriers, decreases strongly on lowering temperature. This explains why low-temperature epitaxy can result in a metastable state corresponding to a DMS with a uniform distribution of magnetic ions. However, growth or annealing at appropriately high temperatures, or the presence of lattice defects, can enhance $M$ and promote TM aggregation.

Because of the interfacial energy, on approaching thermal equilibrium conditions, the size of TM-rich NCs should increase whereas their concentration decrease. However, the formation of a periodic structure by spinodal decomposition at early times may render the Oswald ripening a prohibitively slow process, particularly in DMSs with $\bar{x} \ll 1 / 2$. In contrast, both NCs and the surrounding matrix can attain uniform TM concentrations $x_{2}$ and $x_{1}$, respectively, corresponding to the binodal points, $B_{2}$ and $B_{1}$ in Fig.9 or even a transformation of TM-rich NCs to another crystallographic phase can take place. In either case, by determining $q_{c}$ that maximizes $R(q)$ one can evaluate the expected distance $\lambda_{c}=2 \pi / q_{c}$ between TM-rich NCs,

$$
\lambda_{c}=4 \pi\left[\kappa /\left|2 \partial^{2} F(u) / \partial u^{2}\right|_{0} \mid\right]^{1 / 2},
$$

where $\kappa$, representing the interfacial energy, is of the order of $\left|E_{d}\right| a^{2}$ and $a$ is the lattice parameter. Since the magnitudes of pairing energy $E_{d}$ and $\partial^{2} F(u) /\left.\partial u^{2}\right|_{0}$ are similar (cf. Figs. 1 and 10), the distance between TM-rich NCs is expected to set at about ten lattice constants, the value in reasonable agreement with experimental results collected in Secs.III VIII. Simple geometrical considerations provide an average radius of NCs at given $\bar{x}, x_{1}$ and $x_{2}$, and $\lambda_{c}$. 
Another interesting aspect is the dependence of $R(\vec{q})$ on the crystallographic direction of $\vec{q}$ brought about by the elastic term $\frac{1}{2} k u^{2}$ in $F(u)$ (Hai et al. 2011). According to the theory developed for cubic crystals (Cahn, 1962), if elastic moduli fulfil the relation $2 C_{44}-C_{11}+$ $C_{12}>0$, the spinodal decomposition is predicted to proceed along $\langle 100\rangle$ cubic directions. This inequality is obeyed in zb compounds of interest, GaAs, Ge, and ZnTe. Similar to the case of self-assembling semiconductor quantum dots obtained in the StranskiKrastanov heteroepitaxy regime (Stangl et al., 2004), strain minimization can govern the NC arrangement.

Typically, the TM concentration of the host containing TM-rich NCs is nonzero, $x_{1}>0$. Accordingly, the magnetic response of decomposed alloy shows characteristics of the uniform DMS with TM content $x_{1}$ superimposed on magnetism of a system of NCs with the TM concentration $x_{2}$. Since the crystallographic and chemical structure of the NCs is usually imposed by the host, magnetic properties of the individual NCs may not be listed in the existing materials compendia. Furthermore, magnetism of the NC ensemble is strongly affected by their distribution and coupling, either dipole-dipole type or mediated by strain (Korenev et al. 2015) and/or spins in the host.

\section{SPINODAL NANODECOMPOSITION IN (Ga,Mn)As}

It is well known that (Ga,Mn)As containing uniformly distributed magnetic atoms has become a model system for the entire class of dilute FM semiconductors (Dietl and Ohno, 2014; Jungwirth et al., 2014, Tanaka et al., 2014). Similarly, the decomposed (Ga,Mn)As system, consisting of MnAs or Mn-rich (Mn,Ga)As NCs embedded in Mn-poor (Ga,Mn)As, since its first fabrication (De Boeck et al., 1996, Shi et al., 1996), has revealed properties relevant to the whole family of high $T_{\mathrm{C}}$ semiconductors. For instance, both crystallographic (De Boeck et al., 1996) and chemical (Moreno et al., 2002) phase separations were put into the evidence, depending on fabrication and processing conditions. Furthermore, despite a small diameter down to $2 \mathrm{~nm}$, the ensemble of zb Mnrich NCs shows FM-like features persisting up to $360 \mathrm{~K}$ (Moreno et al. 2002, Yokoyama et al., 2005), to be compared to $T_{\mathrm{C}}$ of $318 \mathrm{~K}$ specific to freestanding samples of MnAs that crystallizes in a hexagonal NiAs-type structure. An enhanced magnetooptical response was found in decomposed films of (Ga,Mn)As (Akinaga et al., 2000a. Shimizu et al. . 2000). The functionalities and device implications of such decomposed films (MnAs NCs in GaAs) are also described elsewhere (Tanaka et al. 2008$)$.

\section{A. Fabrication methods and nanocomposite structure}

Various methods were found to provide decomposed (Ga,Mn)As. It can be obtained by MOVPE (Krug von

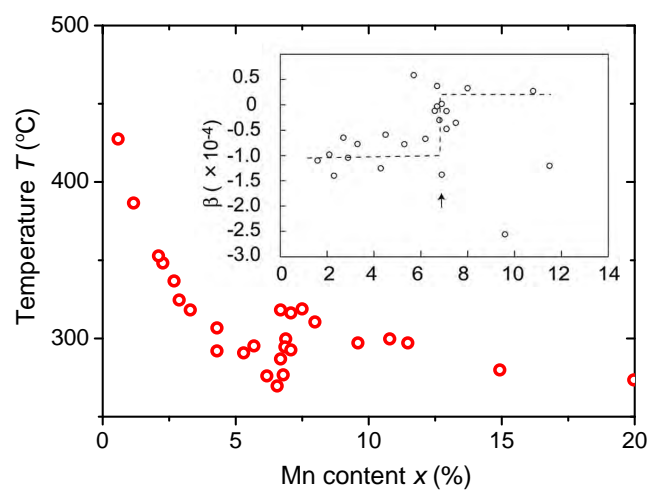

FIG. 13 (Color online) Phase decomposition diagram of (Ga,Mn)As alloy. Open circles are experimental values of temperature $T_{\mathrm{B}}$ at which RHEED streaks, witnessing $2 \mathrm{D}$ growth, change into dots. Inset shows the behavior of the in-plane lattice parameter. Adapted from Hai et al., 2011.

Nidda et al. 2006: Lampalzer et al., 2004) or MBE at sufficiently high substrate temperatures (Hai et al., 2011) or by post-growth annealing of either GaAs implanted with Mn (Ando et al. 1998: Chen et al. 2000, Shi et al. 1996 Wellmann et al. 1997) or fabricated by low-temperature (LT) MBE (De Boeck et al., 1996; DiPietro et al., 2010, Kwiatkowski et al., 2007; Moreno et al., 2002, Rench et al. , 2011; Sadowski et al., 2011; Shimizu et al., 2001; Yokoyama et al. $\mid$ 2005).

At the growth temperature specific to MOVPE, 500$600^{\circ} \mathrm{C}$, one observes assembling of hexagonal MnAs NCs and their segregation toward the layer surface (Krug von Nidda et al. 2006). They have typically elongated shape in the growth direction and their length reaches $100 \mathrm{~nm}$.

According to the MBE growth diagram of $\mathrm{Ga}_{1-x} \mathrm{Mn}_{x}$ As (Dietl and Ohno, 2014, Hayashi et al. 1997. Matsukura et al. 2002, Ohno et al. 1996), at temperatures above $350^{\circ} \mathrm{C}$ at $x=1 \%$ and above $200^{\circ} \mathrm{C}$ at $x=10 \%$ an onset of TM aggregation is observed. Growth temperatures below $200^{\circ} \mathrm{C}$ are required for maintaining 2D growth of (Ga,Mn)As incorporating more than $10 \%$ of uniformly distributed Mn cations (Chiba et al. 2007, Mack et al., 2008, Ohya et al., 2007, Wang et al. , 2008). Results of detailed investigation by reflection high-energy electron diffraction (RHEED) of temperature $T_{\mathrm{B}}(x)$ at which $2 \mathrm{D}$ growth disappears entirely, i.e., RHEED stripes transform into dots, are shown in Fig.13 (Hai et al., 2011). As seen, $T_{\mathrm{B}}(x)$ decreases monotonously with $x$ reaching $270^{\circ} \mathrm{C}$ for $x=6.7 \%$ at which $T_{\mathrm{B}}$ abruptly rises to $320^{\circ} \mathrm{C}$, the effect accompanied by a steplike increase in the in-plane lattice constant. For $x>6.7 \%$ a gradual decrease of $T_{\mathrm{B}}$ continues down to about $250^{\circ} \mathrm{C}$ at $x=20 \%$. This behavior, may indicate that zb-MnAs or Mn-rich (Mn,Ga)As NCs prevail for $x<6.7 \%$, whereas at higher $x$ hexagonal MnAs NCs nucleate at $T \geq T_{\mathrm{B}}$ on the growth surface.

High annealing temperatures, typically $500-700^{\circ} \mathrm{C}$, are 

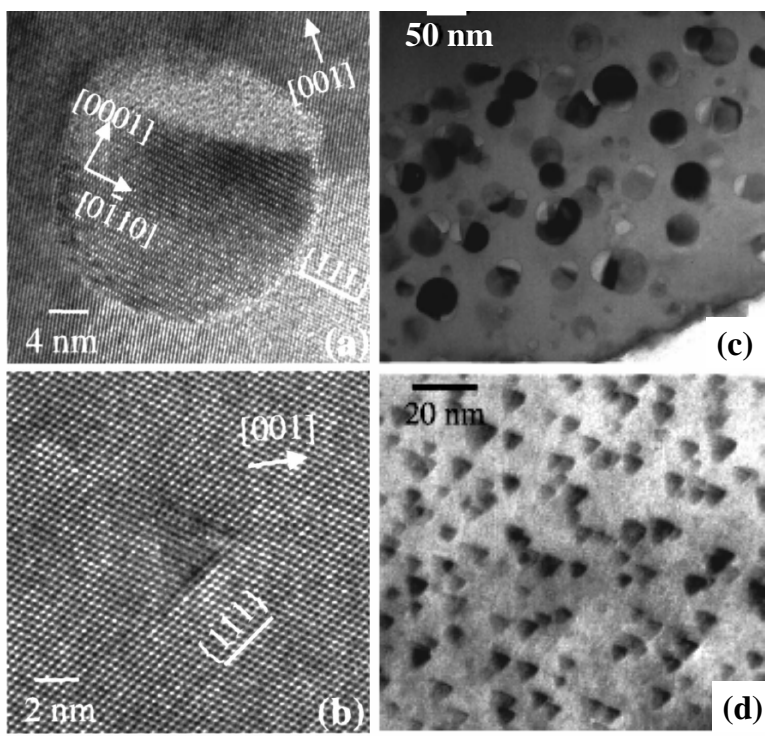

FIG. 14 Evidence for crystallographic and chemical phase separation in (Ga,Mn)As. High-resolution TEM (a,b) and cross-sectional bright-field TEM (c,d) images of $\mathrm{Mn}(\mathrm{Ga}) \mathrm{As}$ clusters embedded in a $\mathrm{Ga}(\mathrm{Mn})$ As matrix for: (a,c) sample I, which was subjected to rapid thermal annealing at $700^{\circ} \mathrm{C}$ and (b,d) sample II, which was annealed in situ at $600^{\circ} \mathrm{C}$ under MBE conditions. The formation of hexagonal MnAs NCs in sample I is the crystallographic phase separation, whereas the zb NCs are the evidence for the chemical separation. From Moreno et al., 2002 .

required to promote ( $\mathrm{Ga}, \mathrm{Mn}) \mathrm{As}$ decomposition in films grown by low-temperature MBE (De Boeck et al., 1996 , DiPietro et al. 2010; Kwiatkowski et al. , 2007; Moreno et al., 2002; Rench et al., 2011; Sadowski et al., 2011, Shimizu et al. , 2001; Yokoyama et al., 2005), although at onset of Mn aggregation was already noted in films annealed at $400^{\circ} \mathrm{C}$ (Sadowski et al., 2011). Similar or higher temperatures were employed to fabricate decomposed (Ga,Mn)As out of Mn-implanted GaAs (Ando et al. 1998, Chen et al., 2000, Shi et al., 1996, Wellmann et al. 1997). These temperatures, significantly surpassing $T_{\mathrm{B}}$ of Fig. 13 collaborate the fact that aggregation of $\mathrm{Mn}$ cations is kinetic limited (see, Sec.II.F), as at given temperature atom diffusion is slower in the bulk than on the growth surface. Furthermore, there is an interfacial energy barrier for forming a nucleus of NCs in the bulk, which requires higher annealing temperatures compared with those formed on the surface (Hai et al., 2011).

Depending on the magnitudes of the initial Mn concentration and annealing temperature, either hexagonal MnAs NCs with an NiAs structure and diameter ranging from 5 to $500 \mathrm{~nm}$ precipitate usually with no dislocations and with orientation $\operatorname{MnAs}(0001) \| \operatorname{GaAs}(111) B$ (crystallographic phase separation) (Ando et al. 1998, Chen et al. 2000, De Boeck et al., 1996; Krug von Nidda et al. , 2006; Moreno et al., 2002, Rench et al., 2011, Shi et al. 1996: Wellmann et al., 1997; Yokoyama et al. 2005) or zb Mn-rich (Ga,Mn)As NCs aggregate (chemi-

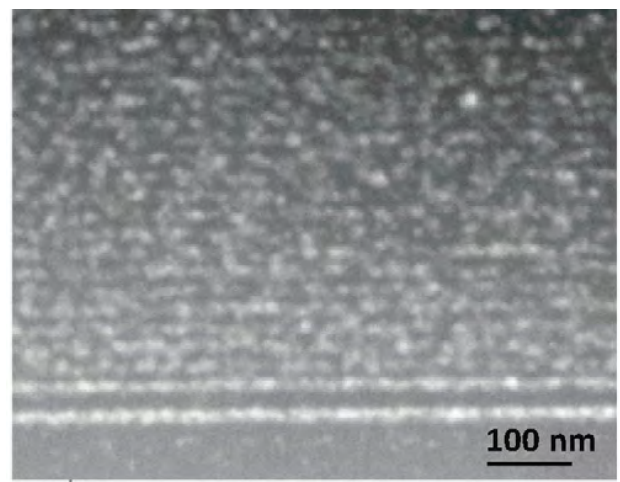

FIG. 15 Cross-sectional STEM (scanning transmittance electron microscope) image of the region close to the $\operatorname{GaAs}(001)$ substrate of a GaAs/(Ga,Mn)As superlattice with Mn content of $3.7 \%$ annealed at $560^{\circ} \mathrm{C}$. Adapted from Sadowski et al. 2013.

cal phase separation) (Kwiatkowski et al. 2007 ; Moreno et al., 2002; Yokoyama et al. 2005), according to highresolution transmission electron microscopy (HRTEM), as shown in Fig. 14. A comprehensive x-ray diffraction (XRD) (Moreno et al., 2003, 2005) and extended x-ray absorption fine structure (EXAFS) studies (Demchenko et al. 2007) of hexagonal MnAs buried in the GaAs lattice provided detailed information on the magnitude of strain in reference to free-standing MnAs. The zb NCs can assume either tetrahedral (Moreno et al., 2002) or spherical form (Kwiatkowski et al., 2007, Sadowski et al. 2011; Yokoyama et al., 2005) with diameter typically from 2 to $6 \mathrm{~nm}$; they are not stable against a transformation to the hexagonal phase for diameters exceeding $15 \mathrm{~nm}$ (Sadowski et al. 2011). Hexagonal and cubic zb NCs coexist in annealed (Ga,Mn)As with Mn content of $2 \%$ and smaller (Sadowski et al., 2011). It appears that the main features of decomposed (Ga,Mn)As and (Ga,Mn)As:Be (Rench et al. , 2011) are similar.

The exact relative concentrations of $\mathrm{Mn}$ and $\mathrm{Ga}$ in the cubic NCs is barely known and may depend on details of the fabrication protocols; the Mn concentration as low as 20\% was suggested (Lawniczak-Jablonska et al. 2011). Under some processing conditions the obtained nanoparticles were found to be structurally disordered (Kwiatkowski et al., 2007; Moreno et al., 2002). In certain cases, presumably if growth of $(\mathrm{Ga}, \mathrm{Mn}) \mathrm{As}$ results in a substantial concentration of As antisites, hexagonal MnAs NCs obtained by annealing are accompanied by As precipitation and voids (Kovàcs et al. 2011 ).

From an application viewpoint particularly relevant is the preparation of magnetically active NCs that reside in a predefine plane. This can be achieved by growing appropriately thin (Ga,Mn)As layers, in which Mn-rich NCs gather after annealing below $600^{\circ} \mathrm{C}$ (Sadowski et al. 2013 Shimizu et al. 2001), as shown in Fig. 15.

Furthermore, according to results presented in Fig.16. rectangularlike distribution of MnAs NCs with a narrow dispersion of diameters was obtained by using the phase 


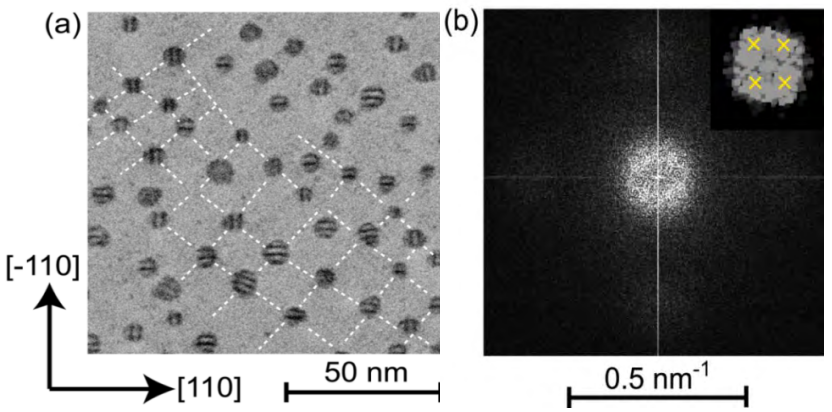

FIG. 16 (Color online) Periodic arrangement of MnAs NCs in GaAs. (a) Plain-view TEM image of hexagonal MnAs NCs fabricated using spinodal decomposition of a $5 \mathrm{~nm}$-thick $\mathrm{Ga}_{0.8} \mathrm{Mn}_{0.2} \mathrm{As}$ layer. (b) Fourier transform of the contrast of a $0.36 \times 0.36 \mu \mathrm{m}^{2}$ TEM image. A ring structure with a radius of $0.063 \mathrm{~nm}^{-1}$ corresponding to a period of $16 \mathrm{~nm}$ is observed. The inset shows the pattern of the ring structure after applying a steplike high pass filter, revealing four fold symmetry, that is, rectangular lattice structure. Adapted from Hai et al. 2011

decomposition diagram of the (Ga,Mn)As alloy and spinodal decomposition induced by annealing.

More recently, the influence of laser irradiation on the self-assembly of MnAs NCs was investigated (Hai et al. 2012). It was found that laser irradiation suppresses the temperature-induced transformation of small into larger hexagonal MnAs NCs, and that the median diameter $D_{1}$ in the size distribution of small NCs depends on the incident photon energy $\hbar \omega$ following $D_{1} \sim \omega^{-1 / 5}$. This behavior was explained by the desorption of Mn atoms from small NCs due to energy gain from optical transitions between their quantized energy levels.

\section{B. Magnetic properties}

Figures 17(a,b) show magnetization loops at various temperatures for (Ga,Mn)As samples with hexagonal and cubic NCs, whose micrographs have been displayed in Figs.14(a,b), respectively. As seen in 17(a), square hystereses pointing to the $T_{\mathrm{C}}$ value specific to bulk MnAs, $T_{\mathrm{C}} \simeq 318 \mathrm{~K}$ are observed for the film with hexagonal NCs (Moreno et al. 2002), the finding also reported by others (Ando et al., 1998, Rench et al., 2011, Wellmann et al., 1997, Yokoyama et al. . 2005). The data indicate that for those large NCs the magnitude of superparamagnetic blocking temperature $T_{\mathrm{b}}=K(T) V / 25 k_{B}$ becomes smaller than the temperature $T$ just below $T_{\mathrm{C}}$. It was also found that $\mathrm{Si}$ codoping enhanced the formation of bigger MnAs nanoclusters (Shimizu and Tanaka, 2002b).

In contrast, the behavior of magnetization in the case of the sample with cubic NCs [Fig. 17(b)] is intriguing and reveals surprising but generic aspects of decomposed magnetically doped systems as follows:

- Despite a rather small size of zb NCs, even at high
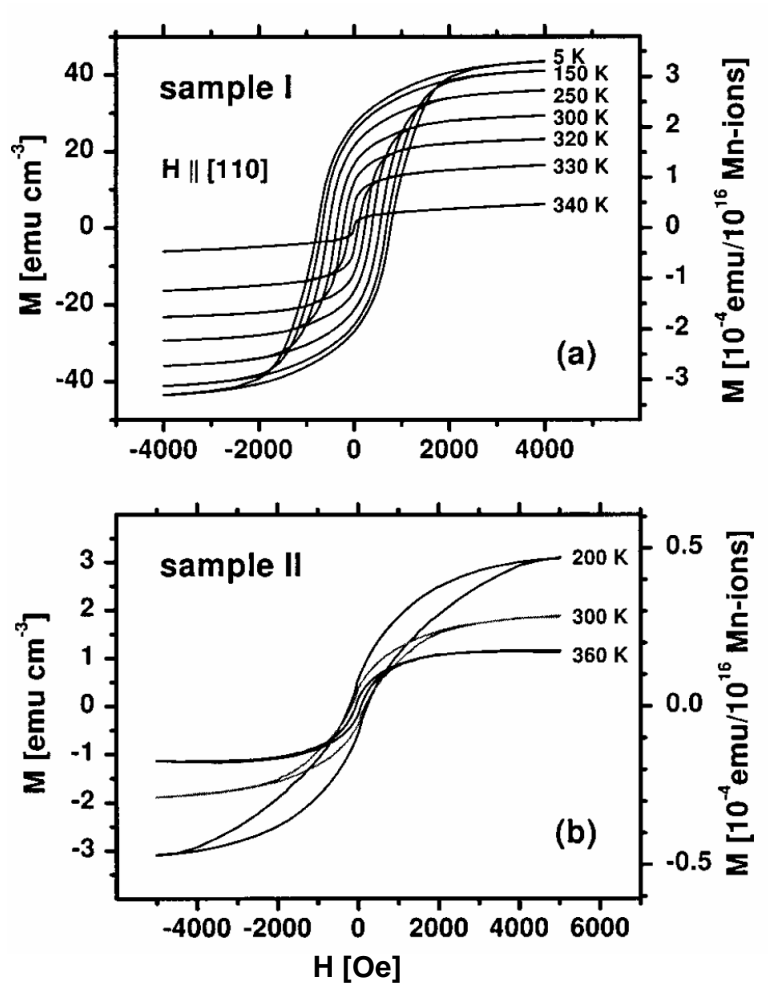

FIG. 17 Magnetization loops at different temperatures of annealed (Ga,Mn)As containing hexagonal (a) and zinc-blende (b) NCs, visualized in Fig.14. From Moreno et al., 2002.

temperatures magnetization loops are opened and weakly temperature dependent. This indicates that $M(H, T)$ cannot be modeled by the Langevin function describing superparamagnetic systems above $T_{\mathrm{b}}$, the conclusion emerging also from other studies of decomposed (Ga,Mn)As (Sadowski et al., 2011 ; Yokoyama et al. , 2005). Given the NC volume $V$, this FM-like behavior points to a rather large magnitude of $K$ or to a significant role played by spin-dependent interactions between the NCs (Hai et al. . 2007). For specific spatial distributions and densities of FM nanoparticles, long-range dipole interactions and/or exchange coupling lead to the so-called superferromagnetic phase (Bolcal et al. 2012, Mørup et al., 2010, Panov, 2012), whose characteristics are consistent with the data displayed in Fig.17(b). In particular, a smaller magnitude of low temperature magnetization, as compared to the value expected from spin counting, is consistent with the superferromagnetic scenario.

- This set of data points to $T_{\mathrm{C}} \gtrsim 360 \mathrm{~K}$, for zb Mn-rich (Mn,Ga)As stabilized by the GaAs host (Moreno et al., 2002, Sadowski et al., 2011, Yokoyama et al., 2005), the value substantially higher than $T_{\mathrm{C}} \simeq 318 \mathrm{~K}$ of free standing $\mathrm{MnAs}$ that crystallizes in the hexagonal structure. This $T_{\mathrm{C}}$ enhancement can be assigned to higher crystal 
symmetry, which typically leads to greater density of states at the Fermi level and, hence, to elevated $T_{\mathrm{C}}$.

- While decomposed films discussed above show superferromagnetic features, in some other samples, containing similar NCs according to TEM studies, a standard superparamagnetic behavior was found (DiPietro et al., 2010, Rench et al., 2011, Sadowski et al. |2011). Differing properties of nominally similar samples imply that magnetic characteristics of decomposed systems are rather sensitive to pertinent details of the system fabrication and the resulting morphology, including the following:

- the chemical structure and composition of the individual NCs (i.e., TM concentration), which determine the electronic structure (e.g., the itinerant versus localized character of $d$ electrons) and, thus, magnetic characteristics such as the magnitude of $T_{\mathrm{C}}$, magnetic moment, and energy of bulk and interfacial magnetic anisotropy

- the spatial distribution and density of the NCs' ensemble, which underlines the magnetic ground state originating from long-range dipole interactions

- the nature of host-mediated spin coupling among the NCs, which can depend on strain (Korenev et al. 2015) and interface between NCs and the host as well as on the TM, carrier, and defect concentrations in the host.

In the case of superparamagnetic samples, the temperature dependence of zero-field cooled magnetization demonstrates that the distribution of NCs' size is lognormal (DiPietro et al. 2010, Rench et al., 2011). At the same time, characteristics of magnetization relaxation are consistent with the superparamagnetic scenario and allow excluding spin-glass freezing as an origin of the magnetization maximum as a function of temperature (Sadowski et al., 2011).

In general, magnetic response of decomposed (Ga,Mn)As contains contributions from both NCs with a high Mn concentration and (Ga,Mn)As with lower Mn content. In the cases discussed previously, the NCs dominate magnetic response in the entire temperature range. However, for $(\mathrm{Ga}, \mathrm{Mn}) \mathrm{As}$ grown by MBE under conditions corresponding to the onset of phase separation, the FM response of the (Ga,Mn)As host can prevail at low temperatures. Here the NCs lead to the pinning of domain walls and to enlargement of the coercive force (Wang et al., 2006 ). Only at high temperatures, above $T_{\mathrm{C}}$ of the host, is magnetization dominated by the NCs (Wang et al., 2006). A similar situation takes place in magnetic resonance studies: FM resonance of MnAs NCs dominates at high temperature, whereas a signal of dilute $\mathrm{Mn}$ ions takes over at low temperatures (Krug von Nidda et al. 2006).

\section{Magneto-optical phenomena}

One of the attractive aspects of decomposed semiconductor alloys is that they show FM characteristics to above RT and at the same time they can be easily integrated with existing semiconductor devices. For instance, one can envisage magnetooptical insulators and modulators that would exploit a large magnitude of the Kerr effect characterizing FM metals and weak optical losses specific to semiconductors. Indeed, the giant Faraday effect and magnetic circular dichroism was found in GaAs with hexagonal metallic MnAs NCs at RT (Akinaga et al. 2000a). The Faraday rotation angle of the GaAs:MnAs layer reached about $0.2^{\circ} / \mu \mathrm{m}$ at $0.98 \mu \mathrm{m}$.

Optical reflectivity of GaAs with hexagonal MnAs and zb-(Mn,Ga)As NCs is similar to that of the host GaAs matrix (Yokoyama et al., 2005). However, magnetooptical spectra and intensities are different in these two kinds of decomposed (Ga,Mn)As; the intensity is weaker in the case zb NCs.

While the magnitude of the Verdet constant (i.e., the normalized Faraday rotation) is attractive and comparable or larger than the one characterizing magnetic materials employed in commercial optical insulators, it is hard to obtain a GaAs:MnAs layer thick enough to insure the required angle of polarization rotation, $45^{\circ}$. However, it is possible to enhance the rotation angle by extending the effective optical path length through a magnetic layer by using multiple reflections. By inserting a 140 nmthick GaAs:MnAs film in between distributed Bragg reflectors (DBR) of GaAs/AlAs, the Faraday rotation angle was enhanced sevenfold at a wavelength of $970 \mathrm{~nm}$ at RT (Shimizu et al. , 2001). The MCD signal at RT as a function of the magnetic field showed saturation at about $1 \mathrm{kOe}$, the dependence corresponding to magnetization of GaAs:MnAs system. Furthermore, a similar structure showed the Kerr rotation of $1.54^{\circ}$ at the designed wavelength of $980 \mathrm{~nm}$ under a relatively low magnetic field at RT (Ueda et al., 2003). Theoretical modeling of optical and magnetooptical properties of these magnetophotonic structures properly describes the experimental data.

As a further step, in order to reduce transmission losses associated with large MnAs NCs, the central GaAs:MnAs layer was replaced by a superlattice (SL) consisting of $2.8 \mathrm{~nm}$-thick AlAs and $5 \mathrm{~nm}$-thick GaAs:MnAs formed by annealing of $(\mathrm{Ga}, \mathrm{Mn})$ As with Mn content of $x=0.047$ (Shimizu and Tanaka, 2001). In this way, the transmission coefficient at a local maximum at $990 \mathrm{~nm}$ was $30 \%$, greatly improved from $2 \%$ in the previous multilayer without a SL. However, in this case, the enhancement in the Faraday rotation per unit magnetic layer thickness, compared to the GaAs:MnAs/AlAs SL structure without DBR, is only 3.3, presumably because of lower structural quality.

Another interesting structure, presented in Fig. 18, was optimized for the photon wavelength of $1.55 \mu \mathrm{m}$ (Shimizu and Tanaka, 2002b). Here, the central $230 \mathrm{~nm}$ thick layer 
consisted of GaAs:MnAs decomposed film codoped with Si donors in order to reduce optical absorption associated with holes delivered by residual $\mathrm{Mn}$ ions in the GaAs host. The figure of merit (FOM), which is defined by the ratio of Faraday ellipticity (shown in Fig 18 ) to optical losses, is $0.074^{\circ} / \mathrm{dB}$ at the wavelength of $1.54 \mu \mathrm{m}$, which is twice as large as the FOM of $0.037^{\circ} / \mathrm{dB}$ at $0.98 \mu \mathrm{m}$ obtained in the studies (Shimizu et al. 2001$)$ referred to previously.

At the same time, properties of waveguide-type optical insulators based on higher losses of backward propagating light due to a nonreciprocal change of the refractive index were theoretically analyzed for an ( $\mathrm{In}, \mathrm{Al}) \mathrm{As}$ waveguide with embedded MnAs nanoclusters (Shimizu and Tanaka, 2002a). Since in this case the proposed optical isolator structure is also composed of all semiconductorbased materials, it can be integrated with III-V based optoelectronic devices such as edge-emitting laser diodes. The device was assumed to be grown on an InP substrate and the operation wavelength was $1.55 \mu \mathrm{m}$. Two designs were considered corresponding to the propagation of TM and TE modes, for which $119 \mathrm{~dB} / \mathrm{cm}$ and $36 \mathrm{~dB} / \mathrm{cm}$ of isolation were predicted, respectively.

\section{Magnetoresistance}

\section{Films}

Transport phenomena in granular metals exhibit a number of outstanding properties Beloborodov et al. 2007) that can be enriched by spin phenomena in decomposed magnetically doped semiconductors (Binns et al. 2005; Michel et al., 2008).

Magnetoresistance (MR) properties of $\mathrm{GaAs} / \mathrm{MnAs}$ granular hybrid structures consisting of FM MnAs clusters within a paramagnetic GaAs:Mn host differ considerably from those of paramagnetic and FM (Ga,Mn)As alloys. According to experimental studies of decomposed samples obtained by various methods, giant positive MR dominates at high temperatures (typically above $\sim 30 \mathrm{~K}$ ) whereas negative MR takes over at low temperatures (Akinaga et al., 2000b; Michel et al., 2005: Wellmann et al., 1998; Ye et al., 2003). Large positive MR was also observed at RT in the impact ionization regime (Yokoyama et al., 2006).

The positive MR effect is similar to the giant MR found in other metal-semiconductor hybrid systems Akinaga et al., 2000b; Solin et al., 2000: Sun et al., 2004). One of relevant mechanisms could be an admixture to the longitudinal voltage of the field-dependent Hall voltage, possibly enhanced by the AHE, a well known phenomenon in other heterogeneous systems (Solin et al., 2000). A strong decrease of the positive MR for the in-plane magnetic field (Ye et al. 2003) supports this scenario.

Two quantum mechanisms, considered widely in the case of homogeneous DMSs (Dietl, 2008b), can contribute to negative MR: (i) an orbital effect originating from the (a)
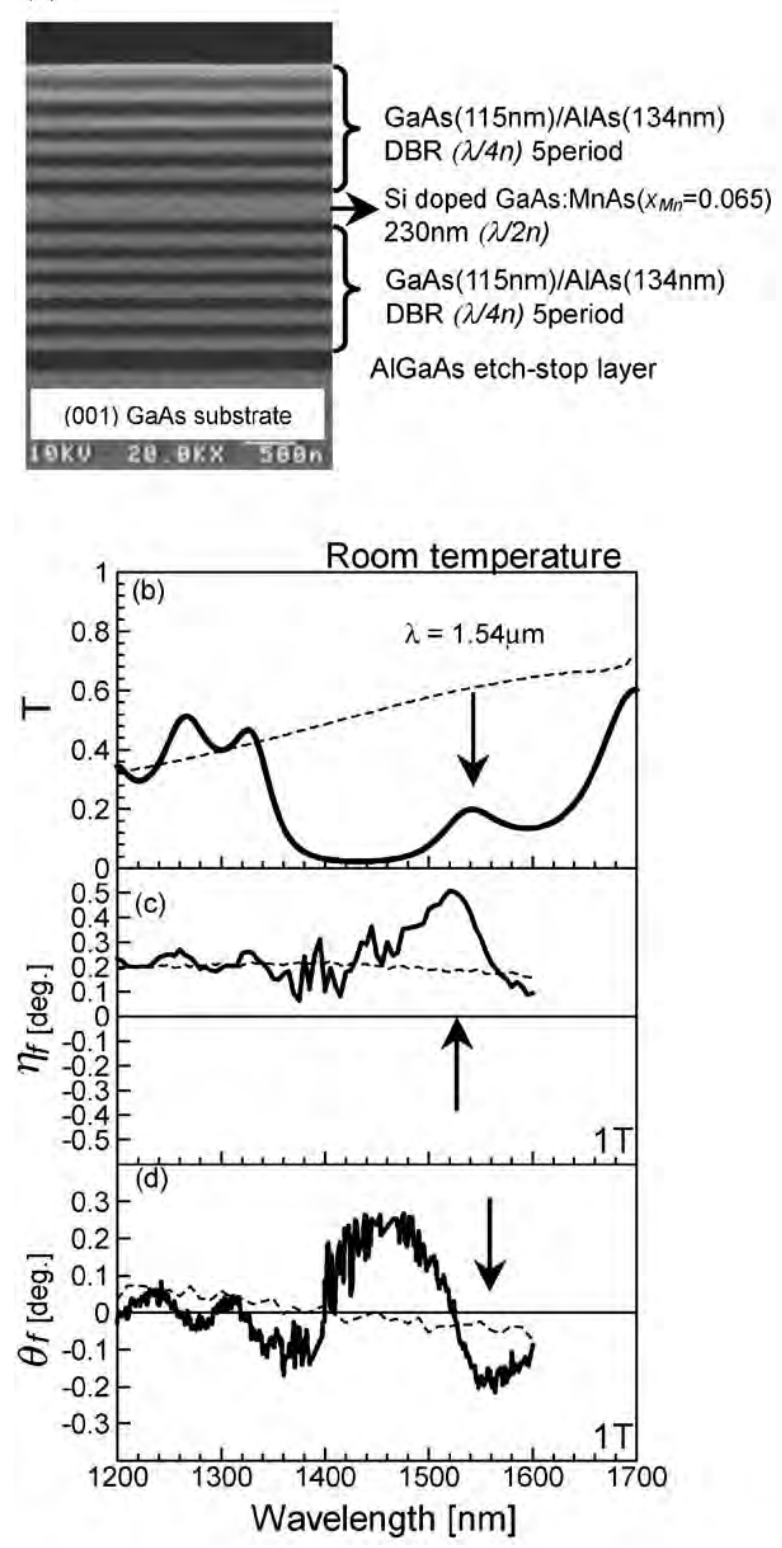

FIG. 18 An optimized structure with MnAs NCs for optical isolation. (a) A cross-sectional micrograph taken by scanning electron microscope of a Si-doped GaAs:MnAs layer placed between DBRs whose operational wavelength was set at $1.55 \mu \mathrm{m}$. The solid lines in $(\mathrm{a}, \mathrm{b}, \mathrm{c})$ show respectively optical transmission $T$; Faraday ellipticity $\eta_{f}$ (transmission MCD) and Faraday rotation $\theta_{f}$ at room temperature in the magnetic field of $1 \mathrm{~T}$ perpendicular to the plane of the sample shown in (a). Spectra of a Si-doped GaAs:MnAs single layer (200 nm) having the same $\mathrm{Mn}$ and Si concentration are also plotted as references (dotted lines). From Shimizu and Tanaka, 2002b

influence of the magnetic field upon interferences of selfcrossing trajectories in either diffusing or hopping regime; (ii) a spin effect brought about by a destructive effect of the magnetic field upon spin-disorder scattering that controls interference of carrier-carrier interaction amplitudes in disordered systems. At the same time, giant MR 
(GMR) or tunneling MR (TMR) effects associated with the field-induced ordering of NCs' magnetic moments or spin-splitting of host's bands produced by stray fields can contribute to the magnitude of MR (Michel et al. 2005). Furthermore, independently of the dominating MR mechanism, the spatial distribution of magnetic NCs is a factor substantially affecting the magnitude of MR and allowing its control (Michel et al., 2008).

\section{Spin-valve structures}

The decomposed (Ga,Mn)As layers containing MnAs NCs with diameter of $10 \mathrm{~nm}$ were also formed in the fully epitaxial magnetic tunnel junctions (MTJs), GaAs:MnAs/Al(Ga)As/MnAs, in which a $20 \mathrm{~nm} \mathrm{MnAs}$ layer constituted the second FM electrode and the barrier was either of AlAs (Hai et al. 2006) or of GaAs (Hai et al. 2008). The magnitude of TMR was examined as a function of temperature and the barrier thickness $d$ attending, at $7 \mathrm{~K}, 17 \%$ for $d_{\mathrm{AlAs}}=2.9 \mathrm{~nm}$ (Hai et al. 2006) and $8 \%$ for $d_{\mathrm{GaAs}}=10 \mathrm{~nm}$ (Hai et al. 2008). The observed oscillatory behavior of the TMR ratio with the increasing AlAs barrier thickness was explained by quantum interference of two X-valley related wave functions in the AlAs barrier. The ensemble of the results demonstrates that GaAs:MnAs layers can act as an efficient spin injector and a spin detector at low temperatures.

Figures 19 $(a, b)$ present micrographs of a lateral nanodevice patterned of a MnAs/GaAs/GaAs:MnAs MTJ, which allowed one to study TMR in the limit of tunneling across a single MnAs NC (Hai et al., 2010). Sizable oscillations in differential conductance $d I_{\mathrm{ds}} / d V_{\mathrm{ds}}$ demonstrated that the Coulomb blockade regime was achieved at $2 \mathrm{~K}$. As shown in Fig.19.(c), clear oscillations were also detected in TMR, defined as a relative difference in the resistance values without an external magnetic field (antiparallel arrangement of magnetization in the MnAs contacts) and in the magnetic field of $1 \mathrm{~T}$ (parallel magnetizations across the device). Modeling of the TMR results by the theory of Barnaś and Fert, 1998 led to the spin relaxation time $\tau_{s}$ of carriers in MnAs NCs as long as $10 \mu \mathrm{s}$. The enhancement of $\tau_{s}$ was assigned to dimensional quantization of electronic states specific to nanoparticles (Hai et al. 2010$)$.

On the other hand, the magnetizing process of $\mathrm{zb}-$ MnAs NCs incorporated into tunnel junctions was found to generate a voltage and a large magnetoresistance effect, which lasted for a time scale of $10^{2}-10^{3} \mathrm{~s}$ (Hai et al. 2009). This spin-motive force was explained by the transformation of magnetic energies of the NCs into the electric energy of the electron system in the Coulomb blockade regime. A similar long-living spin-motive force was observed by Miao et al. 2014 in tunnel junctions with $\mathrm{EuS}$ barriers and $\mathrm{Al}$ nanoparticles. However, an external energy, brought about by, e.g., thermal or electromagnetic noise, should be provided to these systems to maintain such a long-living spin-motive force (Miao et al.
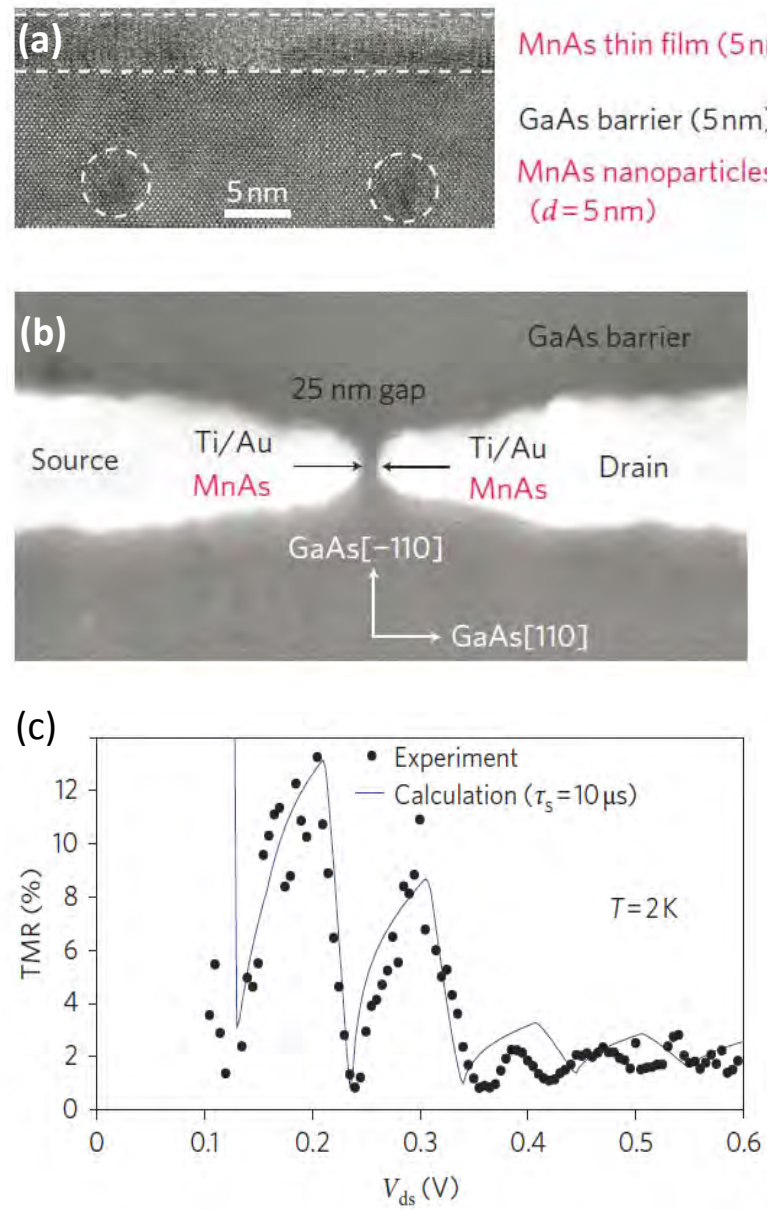

FIG. 19 (Color online) Magnetic tunnel junction involving a single MnAs NC. (a) Cross-sectional TEM image prior to patterning of electrodes and the top-view SEM image of the device (b). The values of TMR $=\left[R\left(\mu_{0} H=0\right)-R\left(\mu_{0} H=\right.\right.$ $1 \mathrm{~T})] / R\left(\mu_{0} H=1 \mathrm{~T}\right)$ as a function of $V_{\mathrm{ds}}(\mathrm{c})$. The line represents the theoretical calculation for carrier spin relaxation time within the MnAs NC $\tau_{s}=10 \mu \mathrm{s}$. Adapted from Hai et al. 2010

2014 Ralph, 2011). The problem of spin-motive force and magnetotransport in nanoscale magnetic systems remains an outstanding experimental and theoretical topic in spinodal-decomposed magnetic semiconductors.

\section{E. Spinodal nanodecomposition in other magnetic III-V compounds}

Several properties outlined previously for decomposed (Ga,Mn)As have been found in other III-V semiconductors doped with TM ions. These systems, if fabricated under specific growth conditions, show FM-like features with $T_{\mathrm{C}}$ magnitudes independent of an average Mn concentration but virtually identical to values characterizing relevant $\mathrm{Mn}$ pnictides, i.e., $T_{\mathrm{C}}$ of 291,318 , and $573 \mathrm{~K}$, for $\mathrm{MnP}$, MnAs, and $\mathrm{MnSb}$, respectively. To 
this category belong (Ga,Mn)P (de Andrés et al. , 2011), (In, Ga, Mn)As/InP (Hara and Fukui, 2006 Hara and Kuramata, 2005), (In,Mn)As (Khodaparast et al., 2013), and (In, Mn)Sb (Lari et al., 2012) grown by MOVPE as well as (Ga,Sb)Mn (Abe et al. , 2000) obtained by MBE and GaP:C implanted with Mn (Theodoropoulou et al. 2002b). A similar situation takes place in Mn-doped II$\mathrm{IV}-\mathrm{V}_{2}$ compounds crystallizing in chalcopyrite structures (Kilanski et al. 2010; Uchitomi et al., 2015). Particularly extensive studies have been performed for magneticallydoped GaN, as discussed in Secs.IV] and V

\section{SPINODAL NANODECOMPOSITION IN (Ga,Mn)N}

\section{A. A controversial system}

Various contradicting information about the magnetism of $(\mathrm{Ga}, \mathrm{Mn}) \mathrm{N}$ has been reported in the literature.

Fabrication of single crystalline $\mathrm{Ga}_{1-x} \mathrm{Mn}_{x} \mathrm{~N}$ epitaxial films with an experimentally documented random distribution of $\mathrm{Mn}$ cations and a small concentration of donorlike defects or residual impurities (Bonanni et al. 2011, Kunert et al. 2012, Sarigiannidou et al., 2006, Stefanowicz et al. , 2010) pointed to the presence of low-temperature ferromagnetism (Sarigiannidou et al. 2006, Sawicki et al., 2012, Stefanowicz et al. 2013). In such samples the Fermi level is pinned by the midgap $\mathrm{Mn}^{2+} / \mathrm{Mn}^{3+}$ impurity band (Dietl and Ohno, 2014, Graf et al., 2003 Wołoś et al. 2009) but $\mathrm{Mn}^{3+}$ ions prevail. A semi-insulating character of this material, exploited as a buffer in recent magnetotransport experiments on GaN:Si layers (Stefanowicz et al., 2014), indicates that owing to strong $p-d$ coupling holes are tightly bound to parent Mn acceptors (Dietl, 2008a; Wołoś et al., 2009). Accordingly, the impurity-band carriers remain localized by, presumably, a combine effect of the Mott-Hubbard and Anderson-Mott mechanism up to at least $x=10 \%$. For the resulting high spin $d$-shell configuration of $\mathrm{Mn}^{3+}$ ions FM superexchange was predicted for tetrahedrally bound DMSs (Blinowski et al., 1996), in agreement with the corresponding experimental results for $\mathrm{Ga}_{1-x} \mathrm{Mn}_{x} \mathrm{~N}$ (Bonanni et al., 2011, Kondo et al., 2002, Sarigiannidou et al. 2006: Sawicki et al. 2012, Stefanowicz et al., 2013). Tight-binding and Monte-Carlo computations carried out within the superexchange scenario and taking into the Jahn-Teller distortion described quantitatively the experimental phase diagram $T_{\mathrm{C}}(x)$ (Sawicki et al., 2012 Stefanowicz et al., 2013). The determined dependence $T_{\mathrm{C}}(x) \propto x^{\alpha}$, where $\alpha=2.2 \pm 0.2$ (Sawicki et al. 2012 , Stefanowicz et al. 2013), characteristic for a short-range superexchange of either sign (Sawicki et al., 2013; Swagten et al., 1992, Twardowski et al. 1987 ), leads to a rather low magnitude of $T_{\mathrm{C}} \lesssim 13 \mathrm{~K}$ at small $x \lesssim 10 \%$ in $\mathrm{Ga}_{1-x} \mathrm{Mn}_{x} \mathrm{~N}$ (Stefanowicz et al., 2013). Within this scenario, ferromagnetism at RT is expected for $x \gtrsim 0.5$, if impurity-band carriers will still remain localized at such high Mn concentrations. In contrast, within the $p-d$ Zener model, long-range FM interactions mediated by band holes would result in $T_{\mathrm{C}}>300 \mathrm{~K}$ at $x$ as small as $5 \%$ in $\mathrm{Ga}_{1-x} \mathrm{Mn}_{x} \mathrm{~N}$ (Dietl et al. 2000).

However, in other series of $\mathrm{Ga}_{1-x} \mathrm{Mn}_{x} \mathrm{~N}$ samples, grown by bulk-like methods with $x \leqslant 0.1$ (Zając et al. 2001 ) or by sputtering that allows $x$ to rise up $36 \%$ (Granville et al. 2010), the interaction between Mn ions was established to be AF. This is consistent with the dominance of $\mathrm{Mn}$ in the 2+ state in those cases (Graf et al., 2003, Granville et al., 2010, Zajac et al., 2001) for which the tight-binding theory mentioned previously predicts AF coupling (Kacman, 2001). A negative sign of the Curie-Weiss temperature, found for MBE-grown films with $x$ between $8 \%$ and $12 \%$ (Dhar et al., 2003b), pointed also to the $\mathrm{AF}$ character of the spin interactions. The presence of spin-glass freezing at $T_{\mathrm{f}}$ between 3 and $4.4 \mathrm{~K}$ was revealed for these samples (Dhar et al. 2003b). The nature of compensating donors providing electrons to $\mathrm{Mn}$ ions has not yet been firmly established though $\mathrm{N}$ vacancies are one of the possibilities (Piskorska-Hommel et al. 2015).

Surprisingly, the appearance of FM-like features in (Ga,Mn)N, persisting up to temperatures well above $300 \mathrm{~K}$, was reported by quite a few groups (Liu et al. 2005: Pearton et al. 2003), and assigned to double exchange that may appear when $\mathrm{Mn}^{3+}$ and $\mathrm{Mn}^{2+}$ ions coexist (Reed et al., 2005; Sonoda et al., 2007). In view of the technological importance of group III nitrides in today's photonics and electronics, the fabrication of a GaN-based functional FM semiconductor would constitute a major breakthrough. Since, however, neither spintronic devices nor phase diagram $T_{\mathrm{C}}(x)$ have so far been reported in this case, it is tempting to assume that the high-temperature ferromagnetism in question is not under control. It is likely that spinodal nanodecomposition was involved in the samples reported to show RT ferromagnetism. Theoretical aspects of nanodecomposition, for which $(\mathrm{Ga}, \mathrm{Mn}) \mathrm{N}$ has often served as a model system, are addressed in Sec.II] No magnetooptical effects associated with high- $T_{\mathrm{C}}$ ferromagnetism have been detected in $(\mathrm{Ga}, \mathrm{Mn}) \mathrm{N}$ (Ando, 2006).

\section{B. Experimental evidences for spinodal nanodecomposition in $(\mathrm{Ga}, \mathrm{Mn}) \mathrm{N}$}

The FM response persisting at elevated temperature and in the absence of carriers mediating the interaction between magnetic ions is presently widely recognized to originate in TM-doped semiconductors from regions of spinodal nanodecomposition in the form of either coherent chemical separation or crystallographic phase precipitation. In the case of $(\mathrm{Ga}, \mathrm{Mn}) \mathrm{N}$ the inclusion of FM NCs with different transition temperatures, such as $\mathrm{Mn}_{4} \mathrm{~N}$ $\left(T_{\mathrm{C}}=784 \mathrm{~K}\right)($ Pop et al. 1994$), \mathrm{Mn}_{3-\delta} \mathrm{Ga}\left(T_{\mathrm{C}}=765 \mathrm{~K}\right)$ (Niida et al. 1996), or $\mathrm{Ga}_{0.8} \mathrm{Mn}_{3.2} \mathrm{~N}\left(T_{\mathrm{C}}=235 \mathrm{~K}\right)$ (Garcia, 1985) has been identified.

As the appearance and the form of spinodal nanode- 
composition depends sensitively on fabrication conditions, codoping by shallow impurities, defect concentration, and post-growth processing, the detection of the subtle presence of phase separation requires a thorough characterization of individual samples with a combination of both local [high-resolution TEM (HRTEM), 3D atom probe (3DAP),...] and averaging [SQUID magnetometry, synchrotron-based XRD (SXRD), x-ray XMCD, magnetotransport,... ] techniques.

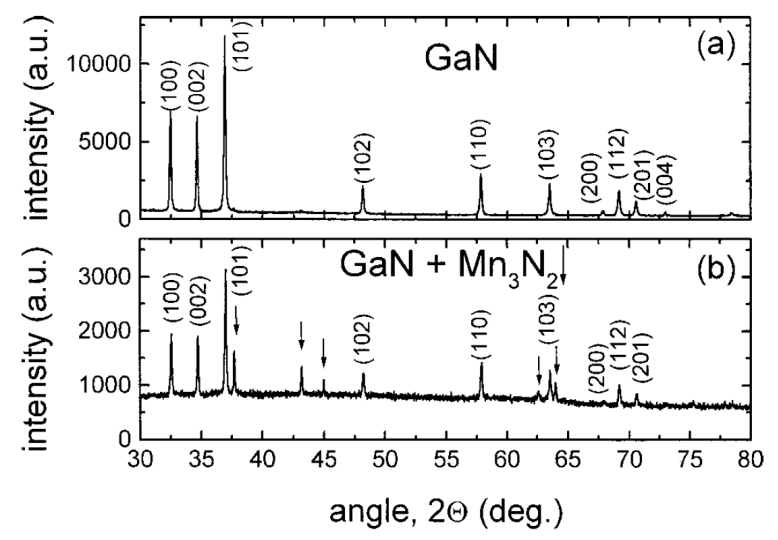

FIG. 20 XRD pattern of (Ga,Mn)N grown by ammonothermal method with (a) low and (b) high Mn content. The Miller indices of the GaN crystal lattice planes are given for each diffraction line and arrows indicate diffraction lines originating from the $\mathrm{Mn}_{3} \mathrm{~N}_{2}$ phase. From Zajac et al., 2001

Early examples of extended characterization of (Ga,Mn)N and detection of crystallographic phase separation are found in works reporting on samples fabricated by means of ammonothermal technique with $0.25 \%$ of $\mathrm{Mn}$ (Zajac et al. 2001). Here, the formation of a $\mathrm{Mn}_{3} \mathrm{~N}_{2}$ was detected through XRD analysis, as reported in Fig.20 and diffraction measurements as in Fig.21 allowed also one to identify $\mathrm{GaMn}_{3} \mathrm{~N}$ phases in MBE layers containing more than 2\% Mn (Giraud et al., 2004, Yoon et al. 2006).

Furthermore, through TEM bright-field micrographs it has been possible to recognize the presence of not better identified secondary phases in MBE (Ga,Mn)N grown onto a GaN buffer and employing specific growth conditions (Kunert et al. 2012) or onto 4H-SiC(0001) substrates and containing $13.7 \%$ of $\mathrm{Mn}$ ions, as seen in Fig.22, where the comparison between TEM micrographs from samples with respectively $7.6 \%$ (left panel) and $13.7 \%$ Mn content (right panel) is given (Dhar et al. 2003a). From this work, the magnetization of the sample with the highest $\mathrm{Mn}$ concentration as a function of temperature and the magnetic field is reported in Fig. 23 . Always worth noting is the fact that the measured magnetization of phase-separated (Ga,Mn)N [and generally (Ga,TM)N] consists of different components, as exemplified in Fig.24 and particular caution should be used to discriminating between the various contributions, to the correct subtraction of the signal from the substrate
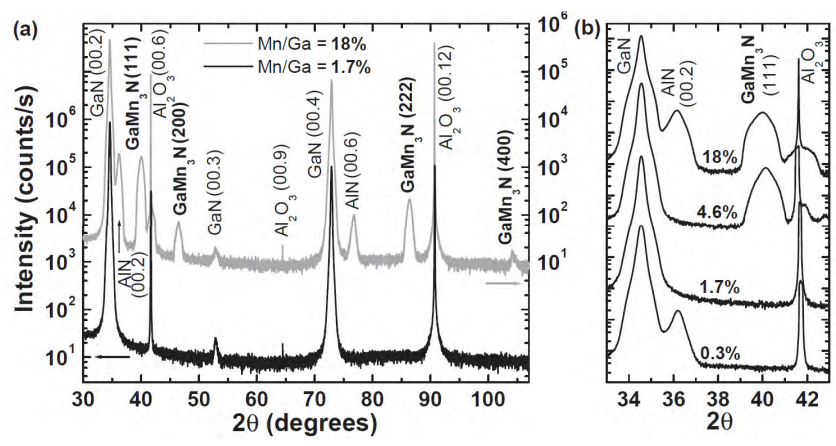

FIG. 21 Determination of NCs' structure in (Ga,Mn)N. (a) XRD patterns of $(\mathrm{Ga}, \mathrm{Mn}) \mathrm{N}$ epilayers. For a $18 \%$ Mn-doped GaN epilayer: presence of oriented clusters with perovskite structure. (b) X-ray absorption: the perovskite clusters at $\mathrm{Mn}$ contents larger than $2 \%$ are identified as $\mathrm{GaMn}_{3} \mathrm{~N}$ NCs. From Giraud et al. 2004.
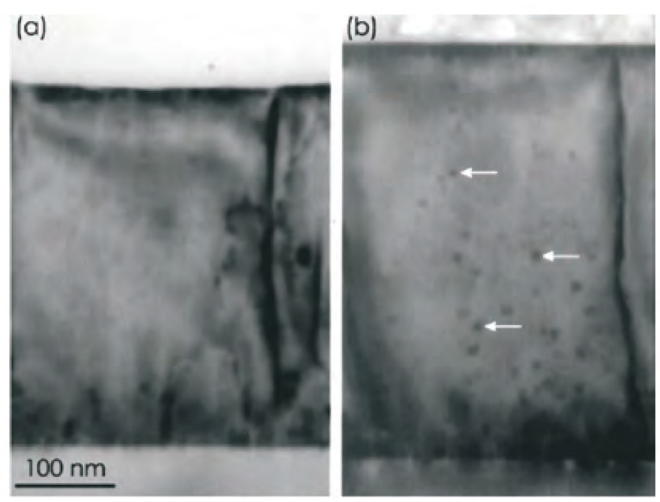

FIG. 22 Bright-field TEM of (Ga,Mn)N with nominal Mn concentrations $7.6 \%$ (a) and $13.7 \%$ (b), respectively. The nmscale clusters are indicated by arrows. From Dhar et al. 2003a

and to possible sources of contamination (Bonanni et al. 2007; Hwang et al., 2007; Ney et al., 2008).

As mentioned, synchrotron radiation-based characterization techniques can supply information when standard methods, such as, e.g., conventional XRD, fail to provide the necessary sensitivity to the presence of spinodal nanodecomposition in the investigated materials (NavarroQuezada et al. , 2010). An example is given by the application of synchrotron radiation microprobe to the identification and study of Mn-rich intermetallic Mn-Ga NCs in $\mathrm{MBE}(\mathrm{Ga}, \mathrm{Mn}) \mathrm{N}$ with $11 \%$ of $\mathrm{Mn}$ (Martinez-Criado et al. . 2005). A combination of fluorescence mapping with spectroscopic techniques allows one to examine the composition of the clusters and their crystallographic orientation, as summarized in Fig.25.

It has been reported that by employing $\mathrm{H}_{2} / \mathrm{N}_{2}$ instead of only $\mathrm{N}_{2}$ during the plasma-assisted MBE (PAMBE) of ( Ga,Mn)N the solubility limit of $\mathrm{Mn}$ can be enhanced and the formation of secondary Mn-rich phases hindered (Cui and Li, 2002). Furthermore, again during a PAMBE 


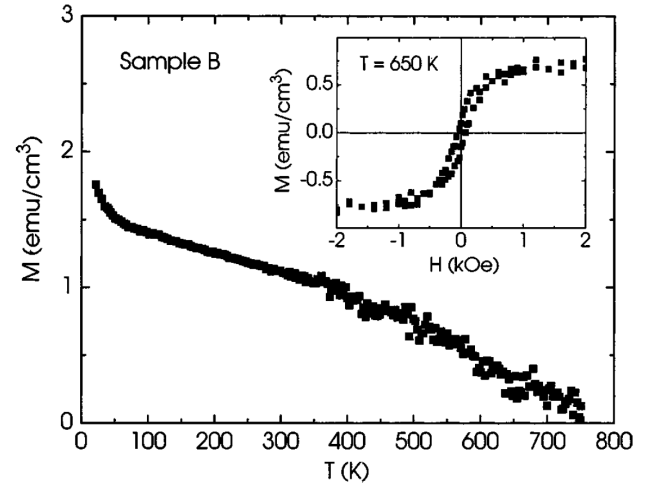

FIG. 23 Magnetization at 50 Oe for a $(\mathrm{Ga}, \mathrm{Mn}) \mathrm{N}$ sample with $13.7 \%$ of Mn. Inset: magnetization loop at $650 \mathrm{~K}$. From Dhar et al. 2003a
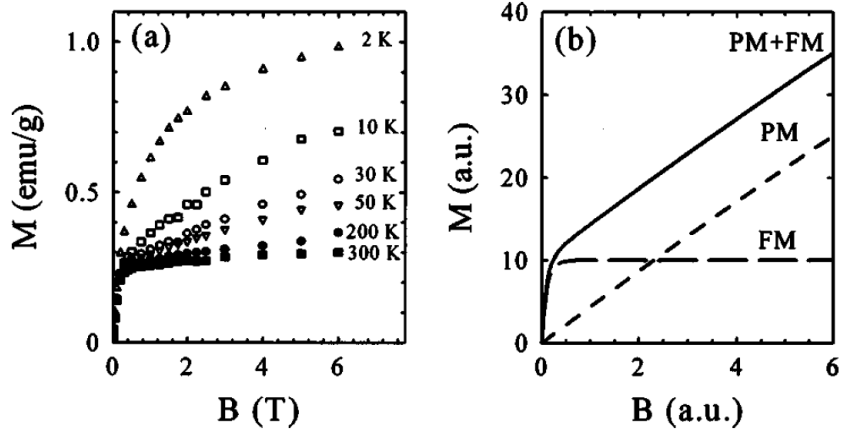

FIG. 24 Evidence for various contributions to (Ga,Mn)N magnetization. (a) Magnetization as a function of the magnetic field for several temperatures. (b) Schematic of the total magnetization (PM + FM) decomposition into paramagnetic (PM) and ferromagnetic (FM) contributions. From Zając et al., 2003.

process, it has been shown that by switching from N-rich to Ga-rich conditions the efficiency of Mn incorporation is diminished, while the crystalline quality is improved and the presence of mixed domains wz-zb is suppressed (Han et al. 2007). The amount of Mn likely to be incorporated without precipitation has been found to depend critically in MBE on the growth temperature $T_{\mathrm{g}}$ (Kondo et al. 2002) and above a critical value of the Mn ions supply, $\mathrm{GaMn}_{3} \mathrm{~N}$ NCs are found to form on the samples surface (Kocan et al. 2006), as exemplified in Fig.26.

The role of defects in the aggregation of $\mathrm{Mn}$ has also been taken into account (Larson and Satpathy, 2007) and from x-ray absorption spectroscopy (XAS) and XMCD data complemented by $a b$ initio calculations it has been concluded that $\mathrm{Mn}$ in GaN preferentially occupies Ga sites neighboring $\mathrm{N}$ split interstitial defects (Keavney et al., 2005). The literature to date lacks a systematic study on the possible role of threading dislocations, especially in a system based on $\mathrm{GaN}$, as preferential site for the aggregation of Mn. Energy-filtered TEM (EFTEM) experiments should be considered, in particular in the
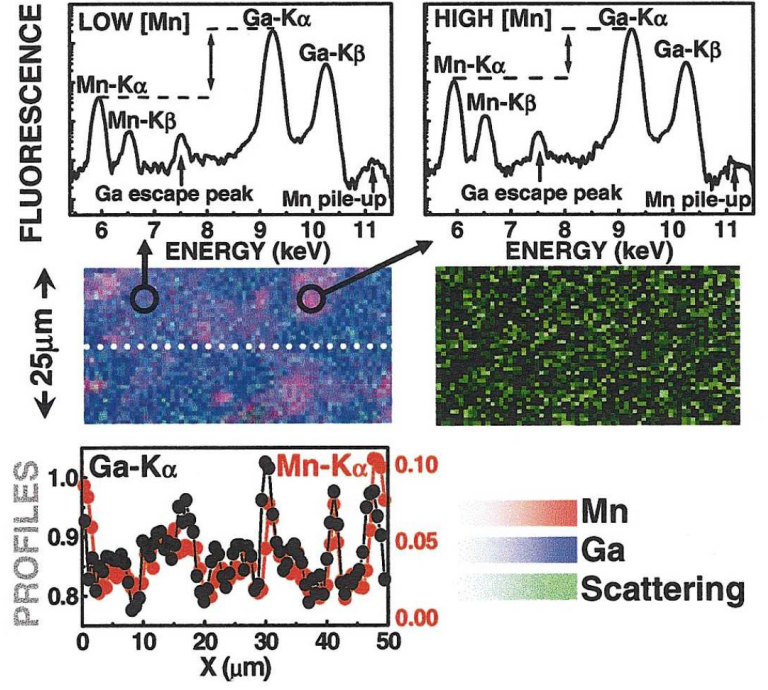

FIG. 25 (Color online) Decomposition of (Ga,Mn)N with an average $\mathrm{Mn}$ concentration of $11 \%$ studied by x-ray fluorescence mapping. Mn $K_{\alpha}, \mathrm{Ga} K_{\alpha}$ fluorescence line, and inelastic (Compton) scattering signal, are shown. Ga and Mn profiles along the dotted scan line are presented in the lower panel indicating the formation of Mn-rich intermetallic MnGa NCs. From Martinez-Criado et al. 2005.

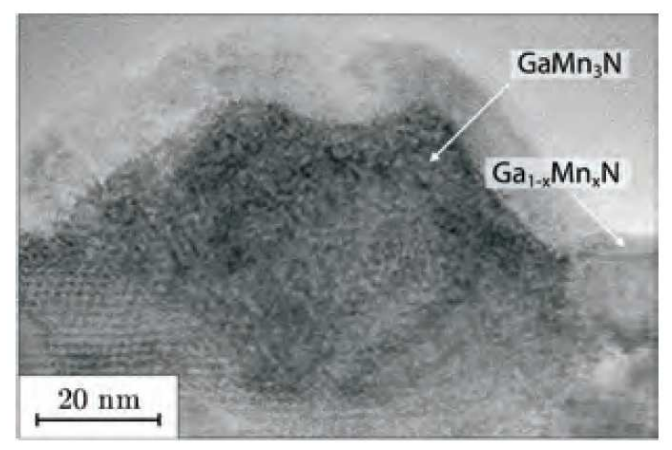

FIG. 26 Cross section HRTEM of a NC in a (Ga,Mn)N layer with nominal $33 \%$ Mn. From Kocan et al. 2006

perspective of identifying coherent regions (not crystallographically phase separated) with enhanced Mn concentration.

The high magnetic moment of Mn and its low solubility limit into GaN, common to all TMs, has motivated some groups to optimize and exploit also Mn-induced phases onto a GaN surface: e.g., magnetic MnGa can grow with an abrupt interface and with a defined epitaxial orientation on wz-GaN, developing an ideal magnetic/semiconductor bilayer ( $\mathrm{Lu}$ et al., 2006). Furthermore, well ordered Mn submonolayers have been deposited onto wz-GaN by evaporation (Chinchore et al. $2008)$ and $(\mathrm{Ga}, \mathrm{Mn}) \mathrm{N}$ nanostructures, though with a notinvestigated structure and actual composition, and grown by MOVPE onto GaN are reported (Gupta et al., 2006). A combined study by scanning tunneling microscopy 


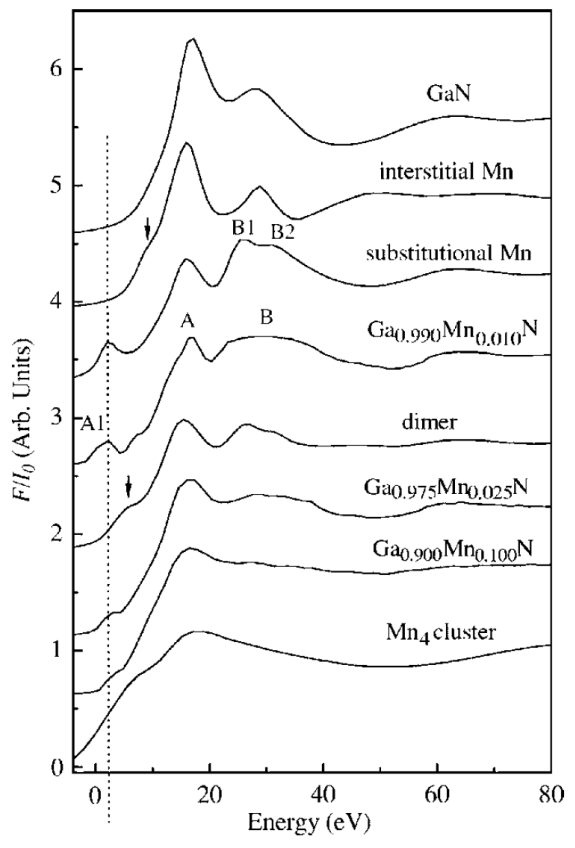

FIG. 27 Comparison of experimental Mn $K$-edge XANES spectra for $\mathrm{Ga}_{1-x} \mathrm{Mn}_{x} \mathrm{~N}$ with various $\mathrm{Mn}$ concentrations with computed spectra assuming various occupation sites in the GaN host crystal. From Wei et al. 2006).

(STM) and first-principles analysis states the feasibility of ordered Mn-induced nanostructures onto GaN(0001) at elevated temperatures, while the growth at RT leads to disordered phases (Qi et al. 2009).

\section{Co-doping with shallow impurities and $\delta$-doping in $(\mathrm{Ga}, \mathrm{Mn}) \mathrm{N}$}

As mentioned previously (Sec.IV.A), the charge state of a magnetic impurity in a semiconducting matrix, which can be altered in GaN by codoping with electrically active impurities, is crucial for the magnetic properties of the material. Moreover, as discussed in Sec.III.B, engineering of the Fermi level position by codoping can serve to modify the lattice position (e.g., interstitial versus substitutional) and the distribution of magnetic impurities over cation sites (Dietl, 2006, Ye and Freeman, 2006).

In addition to infrared and magnetic-resonance spectroscopy (see, Bonanni et al., 2011; Graf et al., 2003, Wołoś and Kamińska, 2008), x-ray absorption near-edge spectroscopy (XANES) is a widely employed method to determine the valence state of $\mathrm{Mn}$ in GaN (Biquard et al. 2003 Bonanni et al., 2011; Sato et al., 2002 | Titov et al. 2005, Wei et al. 2006). As summarized in Fig.27, from the analysis of the absorption-edge prepeaks it is possible to infer the charge state of the TM ions. Furthermore, a careful modeling of the total signal can give information on the presence and nature of phase separation (Biquard et al., 2003, Sato et al., 2002, Titov et al., 2005, Wei et al. 2006). Another sensitive method is x-ray emission spectroscopy (XES) that was exploited to determined the evolution of the Mn oxidation state with the $\mathrm{Mg}$ concentration in (Ga,Mn) N:Mg (Devillers et al., 2012).

The tuning of the Fermi level through codoping with acceptors or donors is expected (Dietl, 2006), and in other systems already proven (Bonanni et al., 2008; Kuroda et al., 2007), to affect the aggregation of TM ions in a semiconducting host and therefore to promote the onset of spinodal nanodecomposition. In $(\mathrm{Ga}, \mathrm{Mn}) \mathrm{N}$ the modulation of the Mn charge state has been mainly considered in relation to its possible effect on the magnetic response. However, the reports are highly controversial: e.g., in some works the reduction from the neutral state $\mathrm{Mn}^{3+}$ to $\mathrm{Mn}^{2+}$ has been found to decrease the FM response of (Ga,Mn)N layers (Yang et al. 2008), while elsewhere it has been argued that paramagnetic $(\mathrm{Ga}, \mathrm{Mn}) \mathrm{N}$ with a majority of $\mathrm{Mn}^{3+}$ can be rendered FM via doubleexchange mechanisms if $\mathrm{Mn}^{3+}$ and $\mathrm{Mn}^{2+}$ coexist in the same sample (Sonoda et al. 2007). Furthermore, codoping with acceptors $(\mathrm{Mg})$ is even reported to act not univocally, but depending on the quality of the matrix to either enhance or quench the FM response (Reed et al., 2005). Again a hint of the crucial importance is a characterization of the material at the nanoscale, as codoping often controls the formation of Mn-rich NCs that can dominate the magnetic properties, especially at high temperatures.

Actually, it was demonstrated that codoping of GaN with $\mathrm{Mn}$ and $\mathrm{Mg}$ during the MOVPE growth leads to the formation of $\mathrm{Mn}-\mathrm{Mg}_{k}$ impurity complexes, where $k$ increases up to $k=3$ with the ratio of $\mathrm{Mg}$ to $\mathrm{Mn}$ concentration according to the binomial distribution (Devillers et al. 2012). These complexes show appealing optical properties, in particular a broad-band infrared emission persisting up to RT (Devillers et al. 2012$)$.

Interestingly, MBE $p$-(Ga,Mn)N spinodally decomposed in regions of $\mathrm{GaMn}_{3} \mathrm{~N}$ is seen to have an enhanced conductivity (Kim et al., 2003 ) that could make this phase-separated system promising for functional effects.

While thermodynamically stable $(\mathrm{Ga}, \mathrm{Mn}) \mathrm{N}$ crystallizes in the wz-structure, it has been reported that MBE layers can be locally stabilized by Mn $\delta$ doping in the zb-phase (Lazarov et al., 2008), as evidenced in Fig.28, Furthermore, the energetics of cubic and hexagonal $(\mathrm{Ga}, \mathrm{Mn}) \mathrm{N}$ arrangements has been studied with $a b$ initio pseudopotential calculations and indeed $\delta$ doping has resulted likely to stabilize the $\mathrm{zb}$ phase (Choi et al. 2006).

\section{SPINODAL NANODECOMPOSITION IN (Ga,Fe)N}

\section{A. Diluted (Ga,Fe)N: current status}

While extensive studies have been conducted on (Ga,Mn)N (Bonanni, 2007; Dietl, 2004: Graf et al., 2003: Liu et al., 2005 (Pearton et al. , 2003) as promising workbench for future applications in spintronics, until very re- 


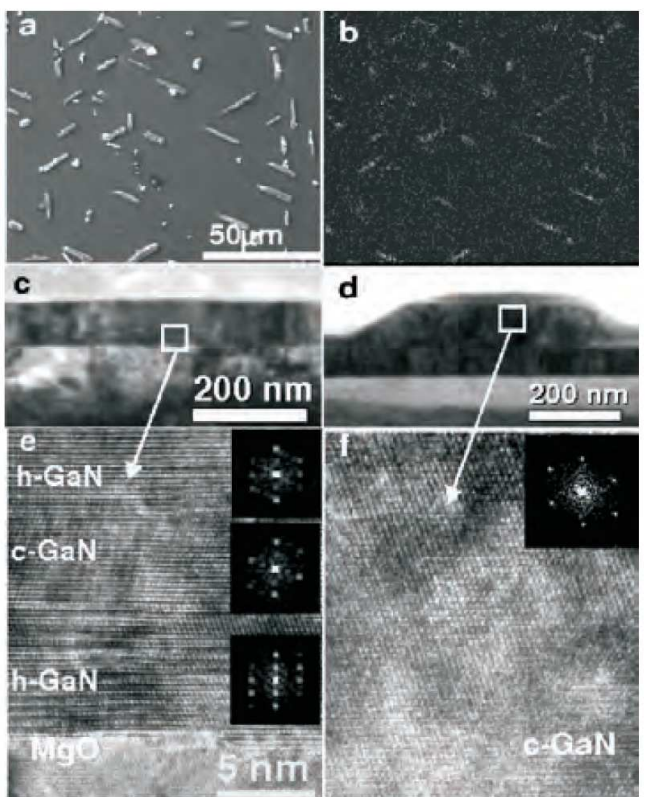

FIG. 28 Multilayer $\mathrm{Mn} / \mathrm{GaN}$ film on $\mathrm{MgO}(111)$ : top view SEM image (a) and Mn EDS map (b) showing 93\% flat film regions and $7 \%$ Mn-rich protrusions. Bright-field TEM (c) and HRTEM (e) images of flat film regions. Bright-field TEM (d) and HRTEM (f) of a Mn-rich protrusion. From Lazarov et al. 2008 .

cently, only little was known about (Ga,Fe)N. Formerly, and to a broad extent still now, this system is being widely considered as semi-insulating substrate for high frequency devices, as AlGaN/GaN high-mobility transistors (Heikman et al., 2003, Kashiwagi et al., 2007; Kubota et al., 2009; Lo et al., 2006; Muret et al., 2007). In this context and generally in relation to the use of this material system in reliable devices, careful studies of the electronic structure of $(\mathrm{Ga}, \mathrm{Fe}) \mathrm{N}$ have been carried out and especially the knowledge of the exact position of the $\mathrm{Fe}^{3+/ 2+}$ acceptor level within the band gap-used to predict band offsets in heterostructures on the basis of the internal reference rule (Langer and Heinrich, 1985) - has been considered of great importance. Furthermore, the behavior of $d^{5}$ and $d^{6}$ level systems in a trigonal crystal field of $C_{3 \mathrm{~V}}$ symmetry is of interest for general aspects of group and crystal-field theory (Lo et al. 2006 , Malguth et al. 2008).

Furthermore, GaN doped with Fe impurities substitutional of $\mathrm{Ga}$ in their $\mathrm{Fe}^{3+} d^{5}$ configuration (Baur et al. 1994; Bonanni et al., 2007, Malguth et al., 2006a, 2008) attracted considerable attention as an ideal system for the study of the $p-d$ exchange interaction in the strong coupling limit (Pacuski et al., 2008). Indeed, magnetically-doped II-VI oxides and III-V nitrides, due to their small bond length and, thus, strong $p-d$ hybridization, are expected to give rise to large values of the exchange energy $N_{0}|\beta|$, a prediction supported by photoemission experiments (Hwang et al. , 2005). Surprisingly, however, abnormally small field-induced exci- ton splittings in paramagnetic (Zn,Co)O (Pacuski et al. 2006), (Zn,Mn)O (Pacuski et al., 2011; Przeździecka et al., 2006$)$, and (Ga,Mn)N (Pacuski et al. , 2007; Suffczyński et al. . 2011) were reported. It was suggested (Dietl, 2008a) that due to the strong $p-d$ coupling, the molecular and virtual crystal approximations fail in oxides and nitrides, making the apparent exchange splitting the valence band, quantified by $N_{0} \beta^{\text {(app) }}$, reduced in absolute value and of opposite sign than expected, and observed in other II-VI DMSs. In this context, measurements of magnetization and magnetoreflectivity in the free exciton region for $(\mathrm{Ga}, \mathrm{Fe}) \mathrm{N}$ epilayers were reported (Pacuski et al., 2008; Rousset et al., 2013), and the obtained values for $N_{0} \beta^{(\text {app })}$ supported the theoretical expectations (Dietl, 2008a).

Moreover, recently reported DFT calculations supporting the notion that the spin-spin coupling in dilute $(\mathrm{Ga}, \mathrm{Fe}) \mathrm{N}$ is $\mathrm{AF}$, while it becomes ferrimagnetic when holes are introduced into the system, have rekindled the discussion on the nature of the magnetic interactions in TM-doped DMS (Dalpian et al., 2009). Additionally, DMSs in general and (Ga,Fe)N in particular, have become model systems to test various implementations of the DFT to disordered strongly correlated systems (Cui et al., 2006, Mirbt et al., 2002, Sanyal et al., 2003, Sato and Katayama-Yoshida, 2002).

\section{B. Fabrication and properties of spinodally decomposed $(\mathrm{Ga}, \mathrm{Fe}) \mathrm{N}$}

Both the above considerations and the search for high$T_{\mathrm{C}}$ DMSs (Dietl et al. 2000 ) impelled the research in various TM-doped GaN, and the Fe-doping has been pursued through a vast palette of fabrication techniques with variegated results. (Ga,Fe)N grown by means of radiofrequency plasma-assisted $\mathrm{MBE}(\mathrm{RF}-\mathrm{MBE})$ at $800^{\circ} \mathrm{C}$ and with a Fe concentration up to $5 \times 10^{21} \mathrm{~cm}^{-3}$ was shown to have superparamagnetic behavior and HRTEM images gave hints of some inhomogeneity in the layers (Kuwabara et al. 2001b).

The same system fabricated by ammonothermal technique (Dwiliński et al. 1998, Gosk et al., 2003) and by a chemical transport method (Gosk et al. , 2003) revealed the coexistence of paramagnetic and FM contributions (see Sec.V.F), but the origin of this latter component was not investigated. The magnetic properties of nominally undoped and $p$-doped Fe-implanted GaN were reported, evidencing a FM response characterized by magnetization hysteresis loops persisting - depending on the provided dose (Pearton et al., 2002$)$ - up to RT (Shon et al. 2004, Theodoropoulou et al., 2002a).

The full width at half maximum (FWHM) of the $\mathrm{GaN}(0002)$ XRD rocking curves peaks acquired on $\mathrm{Fe}$ implanted GaN was found to increase as a function of the Fe dose, though without further analysis guiding to the presence of decomposition (Shon et al., 2006 ). The emission channeling technique applied to Fe-implanted 
(Fe dose up to $10^{19} \mathrm{~cm}^{-3}$ ) GaN samples confirmed the presence of a high percentage of TM ions (up to 80\%) occupying substitutional Ga sites of the host crystal (Wahl et al. 2001). Moreover, GaN films doped with $\mathrm{Fe}$, with concentrations up to $3 \times 10^{19} \mathrm{~cm}^{-3}$ were fabricated by $\mathrm{MBE}$ at growth temperatures $\left(T_{\mathrm{g}}\right)$ ranging from 380 to $520^{\circ} \mathrm{C}$ directly on sapphire (0001) and FM behavior with $T_{\mathrm{C}}=100 \mathrm{~K}$ was observed only in the samples grown at $400^{\circ} \mathrm{C}$ (Akinaga et al. 2000c).

Films of $(\mathrm{Ga}, \mathrm{Fe}) \mathrm{N}$ (Fe density up to $6 \times 10^{21} \mathrm{~cm}^{-3}$ ) fabricated by means of $\mathrm{MBE}$ at $T_{\mathrm{g}}=500-800^{\circ} \mathrm{C}$ showed a superparamagnetic behavior (Kuwabara et al., 2001a b) assigned to Ga-Fe and/or Fe-N inclusions. EXAFS analysis suggests that the decrease of $T_{\mathrm{C}}$ is caused by a structural transition from wz to zb, and this transition was related to the origin of FM in Fe-doped GaN films (Ofuchi et al. 2001). The MOVPE of $(\mathrm{Ga}, \mathrm{Fe}) \mathrm{N}$ was reported, with a focus on the actual Fe content in the layers and its effect onto the carrier concentration (Heikman et al. 2003). MOVPE samples with a Fe-concentration up to $0.7 \%$ molar were claimed to give FM features up to RT even in the absence of holes, but without providing information on the actual distribution of the magnetic ions in the GaN matrix (Gupta et al. 2008; Kane et al., 2007).

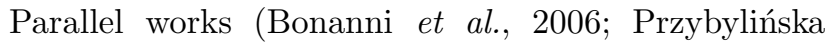
et al. 2006) dealt with the extensive study and characterization of this material system with the precise objective of clarifying the correlation between the general properties - and in particular the magnetic ones - of the system and the distribution of the magnetic ions in the semiconducting matrix.

\section{Fe distribution from nanoscale characterization}

Especially the need to gain insight into the origin of the puzzling FM signatures detected in GaN upon doping with $\mathrm{Fe}$ and persisting up to above RT, has lately prompted a considerable experimental effort in the characterization of the system at the nanoscale. As discussed in this section, $(\mathrm{Ga}, \mathrm{Fe}) \mathrm{N}$ represents an interesting case, in which, for specific growth conditions, NCs formed by spinodal nanodecomposition gather in planes perpendicular to the growth direction.

Early steps were undertaken in the understanding of, e.g., the behavior of Fe-implanted GaN, where a combination of Mössbauer spectroscopy (Fig.29) and HRTEM (Fig. 30 revealed the precipitation of Fe during implantation (Talut et al., 2006). There, ${ }^{57} \mathrm{Fe}$ was employed as an atomic sensitive probe to investigate the local environment of Fe by Mössbauer spectroscopy and to study the precipitation at very early stage. MOVPE $p$-type $\mathrm{Mg}$ doped $10^{17} \mathrm{~cm}^{-3} \mathrm{wz}-\mathrm{GaN}(0001)$ films about $3 \mu \mathrm{m}$ thick grown onto sapphire (0001) substrates were analyzed. The samples were implanted with $200 \mathrm{keV}{ }^{57} \mathrm{Fe}$ ions with fluences between $1 \times 10^{16}$ and $1.6 \times 10^{17} \mathrm{~cm}^{-2}$ for a maximum Fe content of 1-18 at\%.

Conversion electron Mössbauer spectroscopy (CEMS)

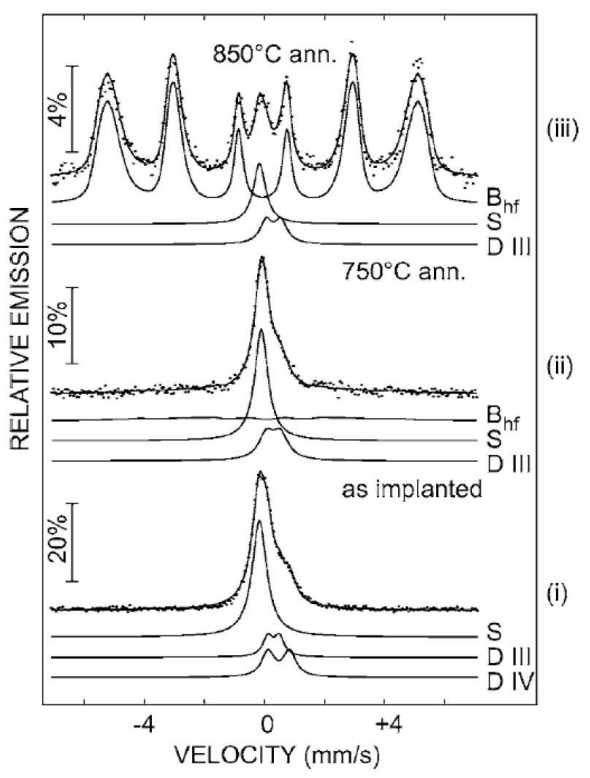

FIG. 29 Room temperature conversion electron Mössbauer spectroscopy spectra of a $4 \times 10^{16} \mathrm{~cm}^{-2}$ Fe-implanted GaN sample: (i) as-implanted; (ii) annealed at $750^{\circ} \mathrm{C}$; (iii) annealed at $850^{\circ} \mathrm{C}$. From Talut et al. 2006

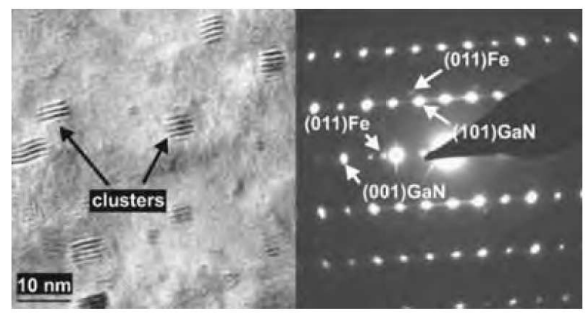

FIG. 30 GaN sample implanted with $4 \times 10^{16} \mathrm{~cm}^{-2}$ Fe ions and annealed at $800^{\circ} \mathrm{C}$ : left panel - HRTEM: Fe clusters in the implanted region of the sample; right panel - selected area diffraction pattern of the same region. From Talut et al. 2006.

in constant-acceleration mode at RT was applied to investigate the Fe lattice sites, electronic configuration, and magnetism. In samples implanted with fluences above $8 \times 10^{16} \mathrm{~cm}^{-2}$, the formation of clusters was observed already in the as-implanted state (Talut et al. 2006). As shown in Fig.29, the CEMS spectra of the as-implanted state were found to consist of a singlet line and two quadrupole doublets. The singlet could be assigned either to superparamagnetic $\alpha$-Fe or to paramagnetic $\gamma$ Fe with an isomer shift of $-0.05 \mathrm{~mm} / \mathrm{s}$. The determined values were assigned to $\mathrm{Fe}^{3+}$ with three $D_{\mathrm{III}}$ and four $D_{\text {IV }}$ nearest $\mathrm{N}$ neighbors with isomer shifts of 0.42 and $0.59 \mathrm{~mm} / \mathrm{s}$, respectively. The magnetically isolated $\mathrm{Fe}^{+3}$ ions were confirmed to be paramagnetic with no FM hyperfine splitting observable.

The presence of the $\mathrm{Fe}^{+3}$ state has been taken as direct proof of its being substitutional of Ga sites in the wz structure, where Ga is surrounded by four $\mathrm{N}$ atoms 


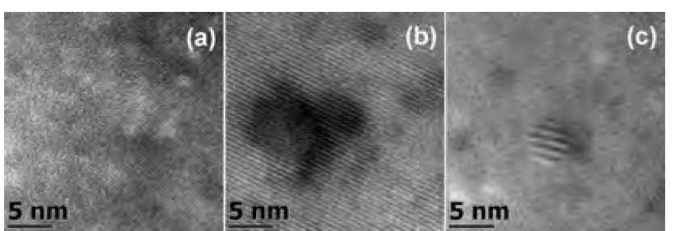

FIG. 31 HRTEM of (Ga,Fe)N showing (a) dilute case; (b) coherent spinodal decomposition (chemical phase separation), and (c) crystallographic phase separation. From Bonanni et al. 2007.

and vice versa. However, only $23.6 \%$ of the total amount of $\mathrm{Fe}$ in the as-implanted samples appeared to substitute the Ga sites. The remanent magnetic ions were regarded to be either on interstitial sites or generating small precipitates of metallic $\gamma$ - or $\alpha$-Fe. Annealing at $850^{\circ} \mathrm{C}$ was found to trigger the formation of $\mathrm{FM} \alpha$-Fe, represented by a sextet with a mean magnetic hyperfine field of $318 \mathrm{kOe}$ reported in Fig.29.

A series of works with the first systematic study of the $(\mathrm{Ga}, \mathrm{Fe}) \mathrm{N}$ system fabricated by means of MOVPE from the impurity limit until the phase separation, was carried out (Bonanni et al., 2007, 2008; Kowalik et al., 2012, Pacuski et al. 2008; Przybylińska et al. 2006; Rovezzi et al. 2009). It was shown that by controlling the growth parameters it is possible to incorporate the magnetic ions in different fashions, giving rise to: (i) a DMS with Fe substitutional of $\mathrm{Ga}$, in the charge state $\mathrm{Fe}^{+3}$ and responsible for the paramagnetic response of the samples; (ii) a system with spinodal decomposition (chemical phase separation) in Fe-rich regions structurally coherent with the matrix; and (iii) a crystallographic phase-separated material consisting of $\mathrm{Fe}_{x} \mathrm{~N}$ NCs embedded in the GaN host and likely to account for the FM signatures, as discussed in Sec.V.F

The different arrangements mentioned are evidenced by the HRTEM images in Fig. 31, where (a) dilute, (b) chemically separated, and (c) crystallographically separated $(\mathrm{Ga}, \mathrm{Fe}) \mathrm{N}$ are, respectively, reported. Energy dispersive x-ray spectroscopy (EDS) measurements could confirm the enhanced concentration of Fe both in the (b) coherent regions and in the (c) incoherent precipitates (Bonanni et al., 2007).

While often laboratory XRD does not evidence any phase separation in $(\mathrm{Ga}, \mathrm{Fe}) \mathrm{N}$ samples giving a FM response - an example is given in Fig.32, where MOVPE $(\mathrm{Ga}, \mathrm{Fe}) \mathrm{N}$ with $0.7 \% \mathrm{Fe}$ ions does not show diffraction peaks from secondary phases - SXRD measurements on MOVPE $(\mathrm{Ga}, \mathrm{Fe}) \mathrm{N}$ with more than $0.4 \% \mathrm{Fe}$, reveal the presence of diffraction peaks identified as the (002) and (111) of the phase $\varepsilon-\mathrm{Fe}_{3} \mathrm{~N}$-as reported in Fig. 33 - a compound known to be FM with $T_{\mathrm{C}}=575 \mathrm{~K}$.

The solubility limit in $(\mathrm{Ga}, \mathrm{Fe}) \mathrm{N}$ was found to be around $0.4 \%$ of the $\mathrm{Fe}$ ions under optimized MOVPE growth conditions (Bonanni et al., 2007). As discussed in Sec.II.B an order of magnitude lower solubility limit of Fe comparing to Mn (see Sec. IV) under similar growth

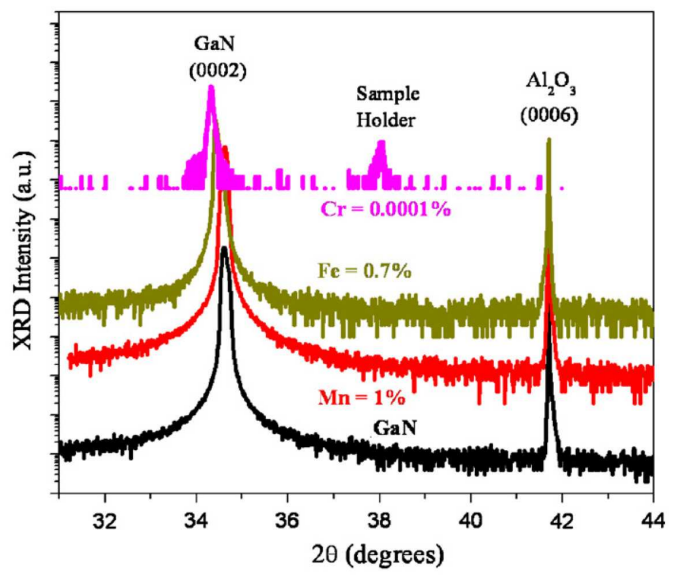

FIG. 32 (Color online) HRXRD for different MOVPE grown (Ga,TM)N. From Gupta et al. 2008.

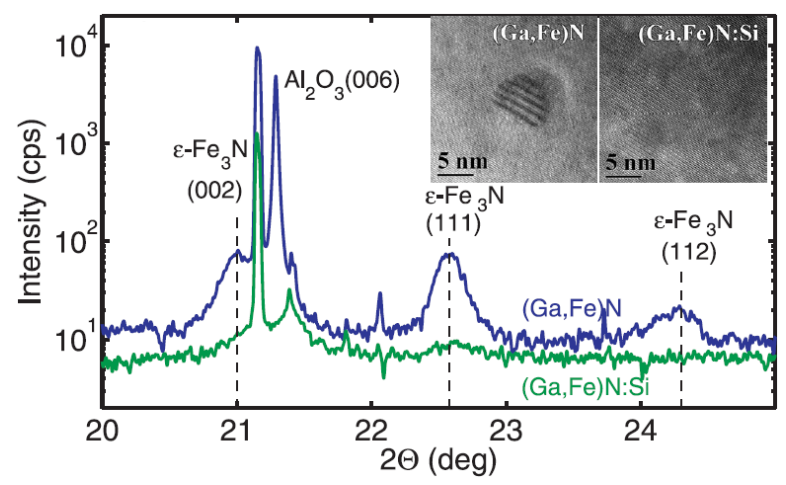

FIG. 33 (Color online) SXRD for MOVPE grown (Ga,Fe)N with Fe density above the solubility limit. Secondary phases identified as $\varepsilon-\mathrm{Fe}_{3} \mathrm{~N}$ are evidenced in accord with HRTEM data (inset). The effect of codoping by Si (see, Sec.V.E) is also shown. Adapted from Bonanni et al. 2008.

conditions is to be linked to a different sign of the chemical forces between TM adatoms on the (0001) GaN surface during the epitaxy: according to ab initio studies the pairing interaction that is repulsive for surface cation $\mathrm{Mn}$ dimers becomes attractive in the case of Fe ions (Gonzalez Szwacki et al., 2011). Actually, a reduced magnitude of FM response with increasing the growth rate Bonanni et al. 2008) and lowering growth temperature (NavarroQuezada et al., 2010) constitutes an experimental hint about the importance of aggregation at the growth surface in the formation of Fe-rich NCs in $(\mathrm{Ga}, \mathrm{Fe}) \mathrm{N}$.

An interesting aspect of spinodal nanodecomposition in $(\mathrm{Ga}, \mathrm{Fe}) \mathrm{N}$ was discovered by HRTEM (NavarroQuezada et al. 2011) and confirmed by x-ray photoemission electron microscopy (XPEEM) and XAS (Kowalik et al. 2012). It was found that Fe-rich NCs tend to accumulate in a plane adjacent to the film surface. This observation allows one to develop a method of controlling the NCs position (Navarro-Quezada et al. 2012 ). As shown in Fig.34, it was demonstrated that for an employed growth mode the NCs gathered in a plane at 


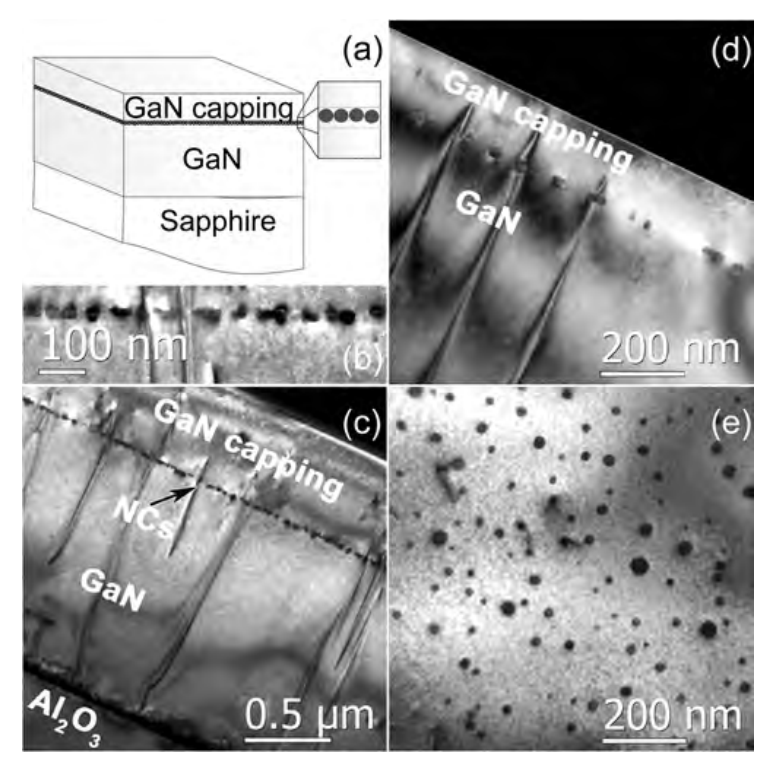

FIG. 34 Control over the spatial distribution of Fe-rich nanocrystals (NCs) in $(\mathrm{Ga}, \mathrm{Fe}) \mathrm{N}$. (a) Schematic layout of the structure; (b) cross-sectional TEM: magnification of the region containing the array of NCs for the sample reported in (c); (c) and (d) cross section TEM images of the samples, showing the spatial distribution of the NCs into a planar array perpendicular to the growth direction and located at the interface to the cap layer, $500 \mathrm{~nm}$ and $150 \mathrm{~nm}$ below the sample surface, respectively; (e) plane-view TEM image of the sample in (c), giving the in-plane distribution of NCs. From Navarro-Quezada et al., 2012).

which the $\mathrm{Cp}_{2} 2 \mathrm{Fe}$ source flow was interrupted, i.e., at the interface between the $(\mathrm{Ga}, \mathrm{Fe}) \mathrm{N}$ layer and the GaN cap. This means that Fe-rich regions move together with the growth front as long as $\mathrm{Fe}$ is supplied, reemphasizing the notion that the aggregation of TM ions occurs at the growth surface.

The command over the location of the NC array is a major step toward applications of decomposed systems. Furthermore, a highly nonrandom distribution of NCs over the film volume indicates that the visualization of spinodal nanodecomposition can be quite challenging, as NCs may reside outside the probed region.

\section{Phase diagram of the spinodal decomposition in $(\mathrm{Ga}, \mathrm{Fe}) \mathrm{N}$}

It is known that an increase of $T_{\mathrm{g}}$ promotes the aggregation of the TM ions incorporated in the semiconductor host and therefore brings the system far from the dilute state. Moreover, various $\mathrm{Fe}_{x} \mathrm{~N}$ phases with specific magnetic and structural properties are expected to be stable up to different temperatures. The MOVPE $(\mathrm{Ga}, \mathrm{Fe}) \mathrm{N}$ material system was studied as a function of $T_{\mathrm{g}}$ in the range between 800 and $950^{\circ} \mathrm{C}$ and for samples with a total concentration of $\mathrm{Fe}$ ions in the range

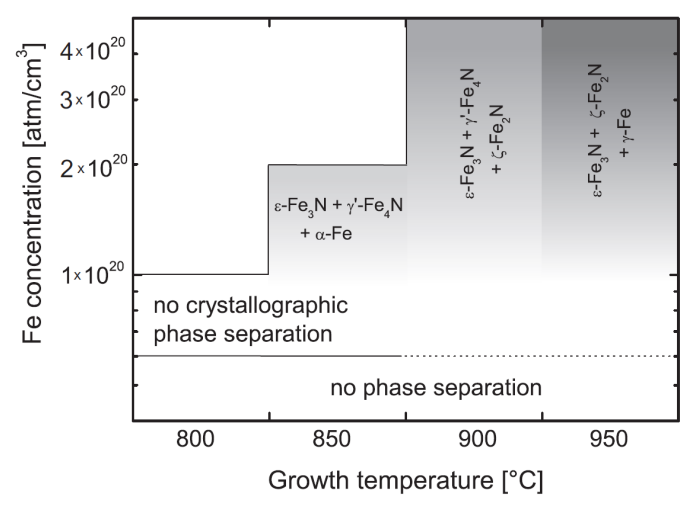

FIG. 35 A phase diagram of MOVPE (Ga,Fe)N as a function of the growth temperature. From Navarro-Quezada et al. 2010

$1-4 \times 10^{20} \mathrm{~cm}^{-3}$ (Navarro-Quezada et al., 2010$)$. In that work, SXRD, EXAFS and XANES, combined with HRTEM and SQUID magnetometry permitted one to detect and to identify particular $\mathrm{Fe}_{x} \mathrm{~N}$ phases in samples fabricated at different $T_{\mathrm{g}}$, as reported in Fig. 35, and to establish establish a correlation between the existence of the specific phases and the magnetic response of the system. It was found that already a $5 \%$ variation in the growth temperature is critical for the aggregations of new $\mathrm{Fe}_{x} \mathrm{~N}$ species and it could be confirmed that an increase in the growth temperature promotes the onset of spinodal decomposition, resulting in an enhanced density of Fe-rich NCs in the matrix and in a consequent increase of the FM response of the system.

In Fig. 36 SXRD spectra for $(\mathrm{Ga}, \mathrm{Fe}) \mathrm{N}$ samples grown at different temperatures, are reported. For layers fabricated at $800^{\circ} \mathrm{C}$ there is no evidence of secondary phases and only diffraction peaks originating from the sapphire substrate and from the GaN matrix are revealed, in agreement with HRTEM measurements showing no crystallographic phase separation. Moving to a $T_{\mathrm{g}}$ of $850^{\circ} \mathrm{C}$ different diffraction peaks belonging to secondary phases become evident, giving proof that at this $T_{\mathrm{g}}$ and at the given growth parameters the system undergoes spinodal decomposition and is phase separated. It was reported (Bonanni et al., 2007) that when growing $(\mathrm{Ga}, \mathrm{Fe}) \mathrm{N}$ at this temperature, one dominant Fe-rich phase is formed, namely wz $\varepsilon-\mathrm{Fe}_{3} \mathrm{~N}$, for which two main diffraction peaks are identified in SXRD, corresponding to the (002) and the (111) reflexes, respectively. A closer analysis of the (111)-related feature and a fit with two Gaussian curves centered at $35.2^{\circ}$ and $35.4^{\circ}$, gives evidence of the presence of the (110) reflex from cubic metallic $\alpha$-Fe. Moreover, the broad feature appearing around $38^{\circ}$ is associated with the (200) reflex of fcc $\gamma^{\prime}-\mathrm{Fe}_{4} \mathrm{~N}$ that crystallizes in an inverse perovskite structure (Jack, 1952).

As the growth temperature is increased to $900^{\circ} \mathrm{C}$ there is no contribution left from the (110) $\alpha$-Fe phase, and the signal from the (111) of $\varepsilon-\mathrm{Fe}_{3} \mathrm{~N}$ is significantly quenched, indicating the reduction in either size or density of the 


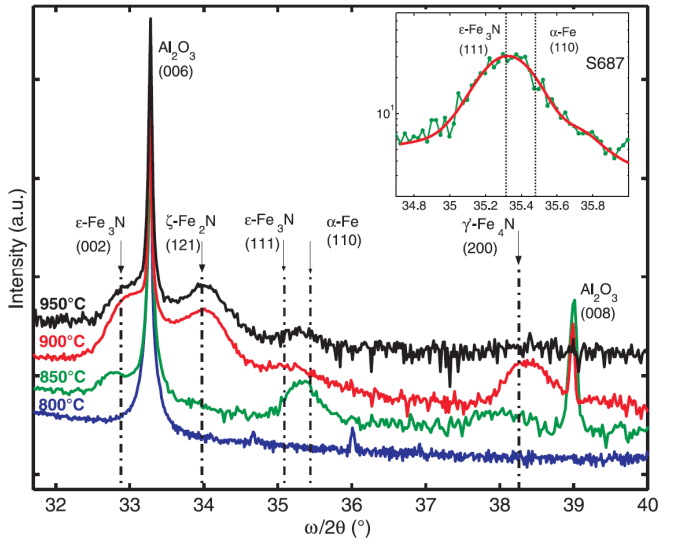

FIG. 36 Color online) SXRD spectra for (Ga,Fe)N layers deposited at different growth temperatures. Inset: peak at $35.3^{\circ}$ deconvoluted into two components assigned to diffraction maxima (111) of $\varepsilon-\mathrm{Fe}_{3} \mathrm{~N}$ and (110) of $\alpha$-Fe [experiment (dotted line) and fit (smooth line)]. From Navarro-Quezada et al. 2010.

specific phase. Furthermore, an intense peak is seen at $34^{\circ}$, corresponding to the (121) contribution from orthorhombic $\zeta-\mathrm{Fe}_{2} \mathrm{~N}$. This phase crystallizes in the $\alpha$ $\mathrm{PbO}_{2}$-like structure, where the $\mathrm{Fe}$ atoms show a slightly distorted hexagonal close packing (hcp), also found for $\varepsilon-\mathrm{Fe}_{3} \mathrm{~N}$ (Jacobs et al. 1995). At a growth temperature of $950^{\circ} \mathrm{C}$ the diffraction peak of $(200) \gamma^{\prime}-\mathrm{Fe}_{4} \mathrm{~N}$ recedes, indicating the decomposition of this fcc phase at temperatures above $900^{\circ} \mathrm{C}$, in agreement with the phase diagram for freestanding $\mathrm{Fe}_{x} \mathrm{~N}$ (Jacobs et al. 1 1995), reporting cubic $\gamma^{\prime}-\mathrm{Fe}_{4} \mathrm{~N}$ as stable at low temperatures. Only the (002) $\varepsilon-\mathrm{Fe}_{3} \mathrm{~N}$ - and the (121) $\zeta-\mathrm{Fe}_{2} \mathrm{~N}$-related diffraction peaks are preserved with a constant intensity and position with increasing temperature, suggesting that at high $T_{\mathrm{g}}$ these two phases and their corresponding orientations are noticeably stable.

Following a procedure based on the Williamson-Hall formula method (Lechner et al., 2009, Williamson and Hall, 1953), the approximate average NCs size is obtained from the FWHM of the diffraction peaks in the radial $(\omega / 2 \theta)$ scans. The FWHM of the $(002) \varepsilon-\mathrm{Fe}_{3} \mathrm{~N}$, of the (200) $\gamma^{\prime}-\mathrm{Fe}_{4} \mathrm{~N}$, and of the (121) $\zeta-\mathrm{Fe}_{2} \mathrm{~N}$ diffraction peaks are comparable for samples grown at different temperatures, indicating that the average size of the corresponding NCs is also constant, as summarized in Fig. 37.

By summing up the SXRD, EXAFS, and HRTEM findings, a phase diagram of the Fe-rich phases formed in (Ga,Fe)N as a function of the growth temperature has been constructed and reported in Fig. 35. showing the dominant phases for each temperature interval. Moreover, according to the $\mathrm{Fe}$ versus $\mathrm{N}$ phase diagram the orthorhombic phase $\left(\zeta-\mathrm{Fe}_{2} \mathrm{~N}\right)$ contains a higher percentage of nitrogen (Jack, 1952) compared to the hexagonal one $\left(\varepsilon-\mathrm{Fe}_{3} \mathrm{~N}\right)$, and this suggests that the higher the growth temperature, the more nitrogen is introduced into the system. Furthermore, similar to the case of (Ga,Mn)As

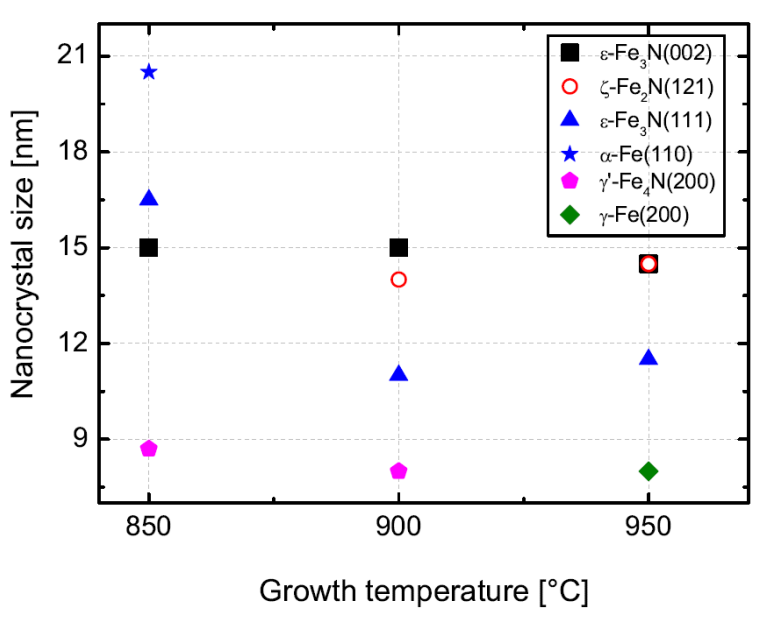

FIG. 37 (Color online) Average size versus growth temperature $T_{\mathrm{g}}$ of nanocrystals in the different $\mathrm{Fe}_{x} \mathrm{~N}$ phases, as determined from SXRD. From Navarro-Quezada et al. 2010.

(III) and (Ga,Mn)N (IV), NCs in (Ga,Fe)N can be built from compounds containing Ga. The presence of planar arrays of $\mathrm{Ga}_{x} \mathrm{Fe}_{4-x} \mathrm{~N}$ NCs was evidenced in a recent work (Navarro-Quezada et al. 2012).

\section{E. Co-doping with shallow impurities in $(\mathrm{Ga}, \mathrm{Fe}) \mathrm{N}$}

Remarkably, HRTEM, SXRD, and SQUID data reveal that the aggregation of Fe ions in a GaN host, and therefore the onset of spinodal decomposition, can be affected by codoping with shallow impurities, $\mathrm{Si}$ donors and $\mathrm{Mg}$ acceptors (Bonanni et al. 2008, Navarro-Quezada et al. 2011).

In Fig.38 HRTEM images data for the two relevant spinodally decomposed initial regimes, namely (i) $(\mathrm{Ga}, \mathrm{Fe}) \mathrm{N}$ with embedded Fe-rich NCs [Fig.38(a)] evidenced by moiré fringes contrast and (ii) (Ga,Fe) N showing coherent chemical separation [Fig. 38(c)] generating mass contrast show the reduced aggregation of the Ferich regions as a consequence of codoping with $\mathrm{Si}$ donors [Fig. $38(\mathrm{~b}),(\mathrm{d})]$.

This effect is further confirmed by the SXRD results given already in Fig. 33 , where the introduction of shallow donor impurities is found to efficiently hamper the precipitate aggregation, so that the SXRD diffraction peaks corresponding to $\varepsilon-\mathrm{Fe}_{3} \mathrm{~N}$ are suppressed in the case of the codoped samples (Bonanni et al., 2008). The quenching of the FM contribution in the codoped layers is further validated by the reduced number of average $\mathrm{Fe}$ ions involved in the FM response in codoped $(\mathrm{Ga}, \mathrm{Fe}) \mathrm{N}$ samples.

In order to explain these key findings, it was noted, as discussed theoretically in Sec.III.B that except for Mn in II-VI compounds (Kuroda et al. 2007), owing to the presence of the open $d$ shells in the vicinity of the Fermi level, the nearest-neighbor pair of TM cations in semiconductors shows a large binding energy that promotes the 


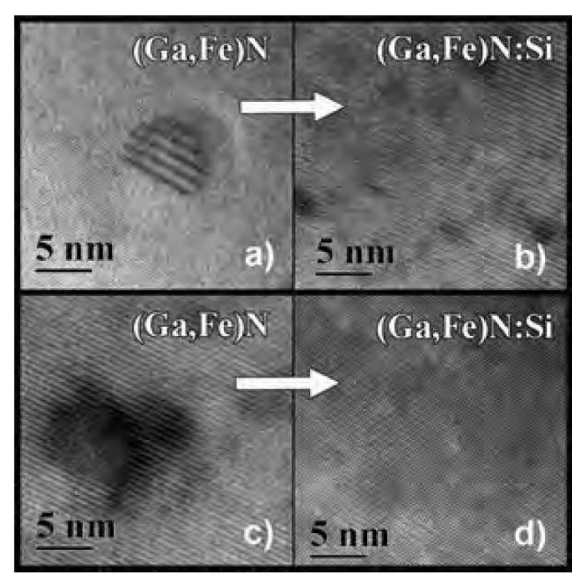

FIG. 38 HRTEM with mass contrast (b,c) of (Ga,Fe)N revealing the presence of $\mathrm{Fe}_{3} \mathrm{~N}$ precipitates (a), spinodal decomposition (c), and the effect of codoping by $\mathrm{Si}(\mathrm{b}, \mathrm{d})$ preventing the formation of the Fe-rich regions. Adapted from Bonanni et al. 2008 .

magnetic ions aggregation. However, as also discussed in Sec.II.B from a theoretical perspective, if carriers introduced by codoping can be trapped by these ions, the pair binding energy will be altered, usually reduced by the corresponding Coulomb repulsion between charged TM impurities (Dietl, 2006, Ye and Freeman, 2006).

The effect of shallow Si donors on the Fe aggregation and consequently on the onset of spinodal decomposition in $(\mathrm{Ga}, \mathrm{Fe}) \mathrm{N}$ is quite clear, since the presence of the midgap electron trap, i.e., the $\mathrm{Fe}^{+3} / \mathrm{Fe}^{+2}$ state, is well established in GaN (Malguth et al. 2008). It was found that the Fe K-edge probed by the XAS shifts under $\mathrm{Si}$ doping from a position expected for the $\mathrm{Fe}^{3+}$ oxidation state toward that specific to the $\mathrm{Fe}^{+2}$ configuration (Rovezzi et al. 2009), as evidenced by the XANES spectra in Fig.339.

In contrast, the role of additional acceptors $(\mathrm{Mg})$ is more complex in $(\mathrm{Ga}, \mathrm{Fe}) \mathrm{N}$. The level $\mathrm{Fe}^{+3} / \mathrm{Fe}^{+4}$ is expected to reside rather in the valence band Malguth et al. 2008), but it was suggested that the potential introduced by a TM ion in GaN (and $\mathrm{ZnO}$ ) is deep enough to bind a hole (Dietl, 2008a), shifting the $\mathrm{Fe}^{+3} / \mathrm{Fe}^{+4}$ up to the GaN band gap (Pacuski et al., 2008). If this is the case, $\mathrm{Mg}$ codoping could also hamper Fe aggregation.

It was found experimentally that the influence of $\mathrm{Mg}$ depends crucially on the way it was introduced to the system: uniform $\mathrm{Mg}$ codoping reduces the probability of incorporation of $\mathrm{Fe}$, diminishing in this way the density of NCs in the samples (Navarro-Quezada et al., 2011). In contrast, codoping of $\mathrm{Mg}$ in the $\delta$ fashion promotes the $\mathrm{NC}$ formation, an effect discussed theoretically in terms of pairing energies of clusters containing different numbers of $\mathrm{Fe}$ and $\mathrm{Mg}$ cations in GaN (Navarro-Quezada et al. , 2011). The influence of $\mathrm{Mg}$ codoping on magnetic properties is discussed below (Sec.V.F.

Based on these results, the previously observed effect of

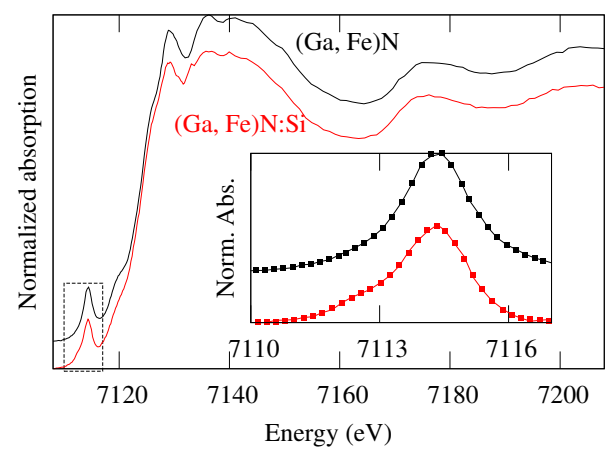

FIG. 39 (Color online) XANES spectra of $(\mathrm{Ga}, \mathrm{Fe}) \mathrm{N}$ and $(\mathrm{Ga}, \mathrm{Fe}) \mathrm{N}: \mathrm{Si}$. The region marked by the dashed box is expanded in the inset. Here the experimental data (lines) after a background subtraction are compared to the fitted values (squares). In addition to the peak at $7114.3 \pm 0.1 \mathrm{eV}$ assigned to the $\mathrm{Fe}^{3+}$ charge state, a shoulder at $7112.7 \pm 0.1 \mathrm{eV}$ is visible in the Si-doped sample pointing to the reduction of a part of the $\mathrm{Fe}$ ions to the $\mathrm{Fe}^{2+}$ charge state by $\mathrm{Si}$ doping. From Bonanni et al. 2008

codoping on ferromagnetism in $(\mathrm{Ga}, \mathrm{Mn}) \mathrm{N}$, and assigned to the dependence of the double-exchange mechanisms of the spin-spin coupling on the position of the Fermi level with respect to the center of the $d$ band (Reed et al. 2005), has to be reconsidered.

\section{F. Magnetic properties of $(\mathrm{Ga}, \mathrm{Fe}) \mathrm{N}$}

The comprehensive nanocharacterization of $(\mathrm{Ga}, \mathrm{Fe}) \mathrm{N}$ allows to put on a more firm basis the origin of a complex magnetic response reported repeatedly for this system. Two examples are given in Figs. 40 and 41, in which various contributions to magnetization are clearly seen. The richness of the magnetic response correlates with the multiphase character of $(\mathrm{Ga}, \mathrm{Fe}) \mathrm{N}$ with Fe concentrations beyond the solubility limit, as discussed in Secs.VC-E.

In general, according to studies of magnetization (Bonanni et al., 2007, Gosk et al., 2003, Navarro-Quezada et al., 2011, 2010), electron paramagnetic resonance (EPR) (Bonanni et al., 2007, Malguth et al., 2006a), ferromagnetic resonance (FMR) (Grois et al., 2014), infrared spectroscopy (Malguth et al., 2006a b), magnetooptics (Pacuski et al., 2008; Rousset et al., 2013), and XMCD (Kowalik et al., 2012) Fe dopants appear in distinct magnetic phases in $(\mathrm{Ga}, \mathrm{Fe}) \mathrm{N}$, whose relative importance depends on growth conditions, Fe concentration, and codoping by donors or acceptors.

Brillouin paramagnetism of Ga-substitutional $\mathrm{Fe}^{3+}$ ions: Because of a nonzero but relatively low solubility limit, a paramagnetic contribution from diluted $\mathrm{Fe}^{3+}$ ions is always present (provided that the density of compensating donors is sufficiently small), as confirmed by a quantitative interpretation of EPR spectra in terms of weakly interacting localized spins $S=5 / 2$ occupying Gasubstitutional sites (Bonanni et al., 2007; Malguth et al. 


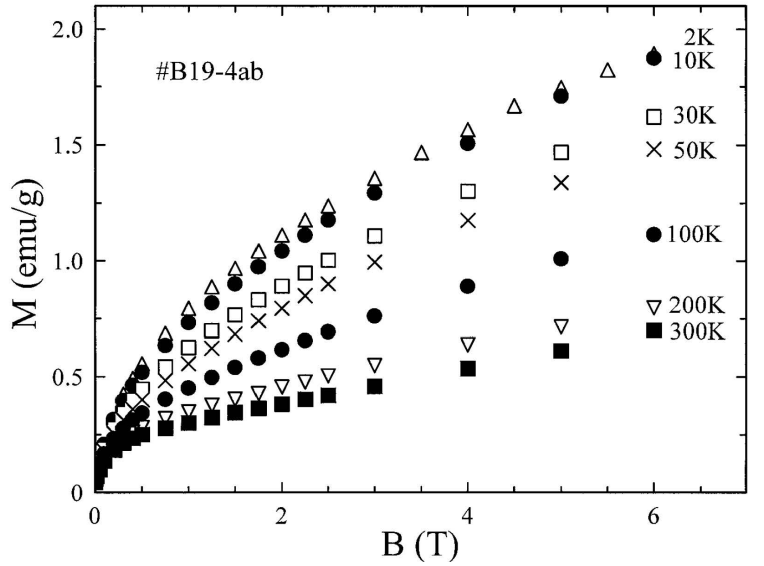

FIG. 40 Magnetization of $(\mathrm{Ga}, \mathrm{Fe}) \mathrm{N}$ bulk crystals obtained by a chemical transport method showing a nonsaturating linear in field component assigned to a Van Vleck-type paramagnetism of $\mathrm{Fe}^{2+}$ ions, together with a high- $T_{\mathrm{C}}$ ferromagnetic contribution found in weak fields. From Gosk et al. 2003.

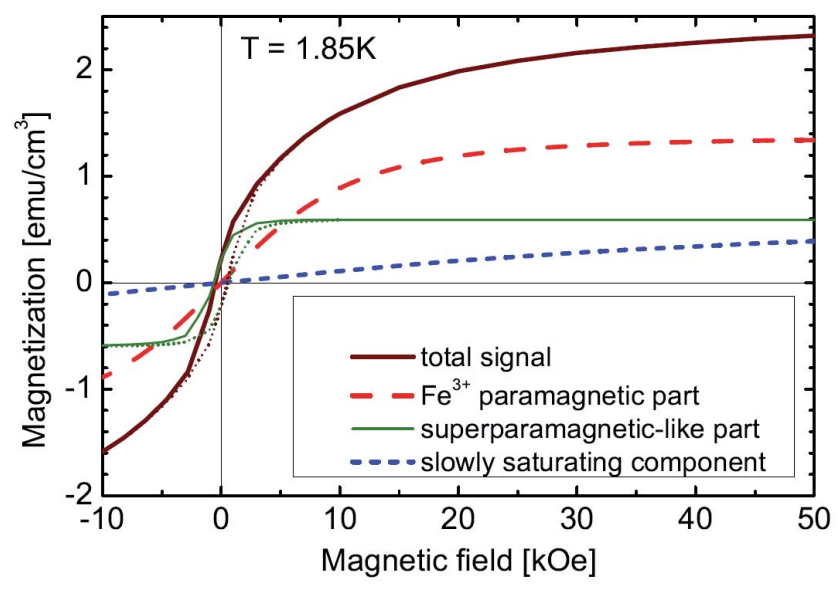

FIG. 41 (Color online) Crystallographically phase-separated ( $\mathrm{Ga}, \mathrm{Fe}) \mathrm{N}$ - contributions to the total magnetization as a function of the magnetic field : (i) paramagnetic from noninteracting $\mathrm{Fe}^{3+}$, (ii) high- $T_{\mathrm{C}}$ superparamagneticlike or superferromagneticlike from the FM NCs and (iii) slowly saturating component, presumably coming from AF NCs. From Navarro-Quezada et al. 2010

2006a). These spins give rise to the Brillouin-like dependence of magnetization on the magnetic field and temperature visible in magnetooptical (Pacuski et al., 2008, Rousset et al. 2013) and magnetization measurements (Bonanni et al. 2007, 2008, Navarro-Quezada et al. 2010. Pacuski et al. 2008).

Van Vleck paramagnetism due to $\mathrm{Fe}^{2+}$ ions: In addition to the Brillouin-like term described previously, a term linear in the magnetic field was found to contribute to $(\mathrm{Ga}, \mathrm{Fe}) \mathrm{N}$ magnetization, and assigned to the Van Vleck paramagnetism of $\mathrm{Fe}^{2+}$ ions that can be present in $(\mathrm{Ga}, \mathrm{Fe}) \mathrm{N}$ due to residual or purposely introduced donors (Bonanni et al., 2007, Gosk et al., 2003, Malguth et al.,

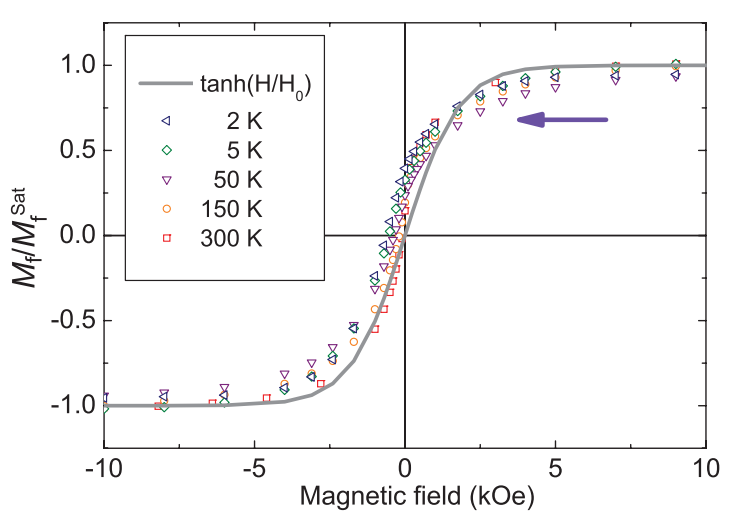

FIG. 42 (Color online) Normalized magnetization at various temperatures for $(\mathrm{Ga}, \mathrm{Fe}) \mathrm{N}$ codoped by $\mathrm{Mg}$ in $\delta$-like fashion with embedded $\mathrm{Fe}=$ rich nanocrystals. Points: experimental data; solid line: fit a Langevin-type function with $H_{0}=800$ Oe; arrow: sweep direction of the magnetic field. From Navarro-Quezada et al. 2011.

\section{6b).}

High- $T_{C}$ ferromagnetism: A FM-like contribution to magnetization of $(\mathrm{Ga}, \mathrm{Fe}) \mathrm{N}$, dominating at high temperatures, shows characteristics specific to the whole family of high- $T_{\mathrm{C}}$ DMSs and dilute magnetic oxides (Coey et al. 2010: Sawicki et al. 2013). As presented in Fig.42, for any temperature and orientation of the magnetic field magnetic hysteresis loops $M(H, T)$ are leaning and narrow, so that the magnitude of spontaneous magnetization $M_{s}(T)$ is much smaller than the saturation magnetization $M_{\text {Sat }}$. Remarkably, $M_{s}(T)$ persists up to above RT and the values of normalized magnetization are approximately described by a temperature-independent Langevin function $M(H) / M_{\text {Sat }}=\tanh \left(H / H_{0}\right)$, where in the case under consideration $H_{0} \simeq 800$ Oe.

This behavior is in contrast to the one of $(\mathrm{Ga}, \mathrm{Mn}) \mathrm{As}$, which is characterized by a squarelike shape and and strong temperature dependence of hystereses. At the same time, if interpreted in terms of superparamagnetism it indicates that, for some NCs, the blocking temperature $T_{\mathrm{b}}$ is higher than RT. This conclusion was confirmed via zero-field cooled (ZFC) and field cooled (FC) SQUID magnetometry measurements at a low magnetic field of 50 Oe (Bonanni et al. 2007), as shown in Fig.43. However, the high magnitudes of $T_{\mathrm{b}}$ were surprising in view of the relatively small values of $\mathrm{NC}$ diameters, shown in Fig. 37.

It was suggested that dipole-dipole interactions between densely packed magnetic constituents may lead to a magnetization that can be parametrized by a temperature-independent Langevin-type function (Coey et al. 2010), a behavior refer to as superferromagnetism (Sawicki et al. 2013). According to TEM (NavarroQuezada et al. 2010) and XPEEM (Kowalik et al. 2012), NCs tend to aggregate in planes perpendicular to the growth direction in the case of $(\mathrm{Ga}, \mathrm{Fe}) \mathrm{N}$, which increases 


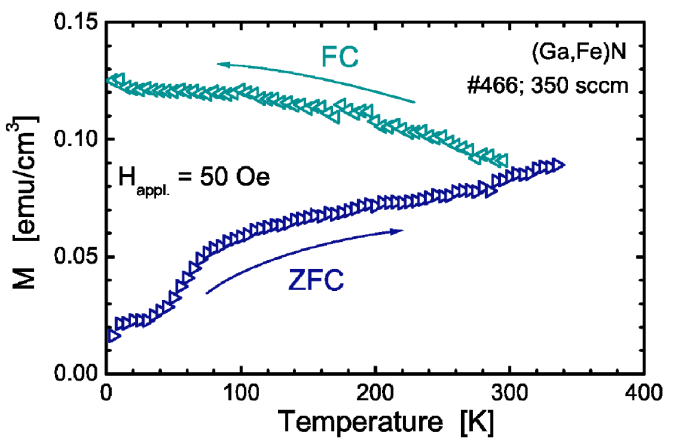

FIG. 43 (Color online) ZFC-FC magnetization at $H_{\text {appl. }}=$ 50 Oe for a $(\mathrm{Ga}, \mathrm{Fe}) \mathrm{N}$ sample with crystallographic phase separation. From Bonanni et al., 2007

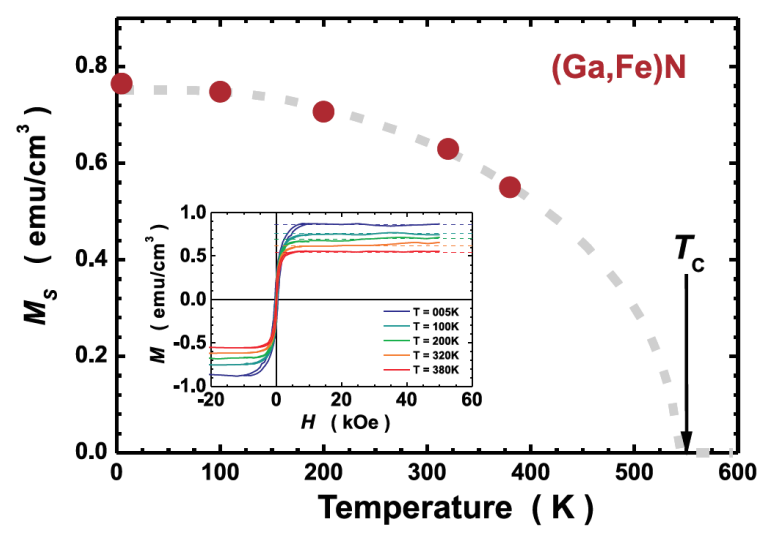

FIG. 44 (Color online) Saturation magnetization determined from the Arrott plots as a function of temperature for spinodally decomposed $(\mathrm{Ga}, \mathrm{Fe}) \mathrm{N}$ : the evaluated $T_{\mathrm{C}}$ of $575 \mathrm{~K}$ corresponds to the Curie temperature of $\varepsilon-\mathrm{Fe}_{3} \mathrm{~N}$. Adapted from Bonanni et al., 2007

the density of NCs and thus supporting the superferromagnetism scenario (Navarro-Quezada et al., 2011).

This interpretation requires also that the $T_{\mathrm{C}}$ of individual NCs is well above RT. According to the results presented in Fig. 44, the value of $M_{\text {Sat }}$ determined from Arrott plots as a function of temperature for the same type of samples, pointed to a $T_{\mathrm{C}}=575 \mathrm{~K}$. This value is in accord with the identification of the dominant secondary phases via SXRD and TEM as $\varepsilon-\mathrm{Fe}_{3} \mathrm{~N}$ in the samples under consideration (Bonanni et al. 2007). According to the phase diagram discussed in Sec.V.D, the abundance of ferromagnetic NCs $\left(\varepsilon-\mathrm{Fe}_{3} \mathrm{~N}, \gamma^{\prime}-\mathrm{Fe}_{4} \mathrm{~N}, \alpha^{\prime}-\mathrm{Fe}\right.$, and their derivatives) increases with lowering the epitaxy temperature.

Recently, FMR was detected at RT in spinodally decomposed $(\mathrm{Ga}, \mathrm{Fe}) \mathrm{N}$ in which $\mathrm{Ga}_{x} \mathrm{Fe}_{4-x} \mathrm{~N}$ NCs formed a planar array (Grois et al. , 2014). The magnetic anisotropy was found to be primarily uniaxial with the hard axis normal to the NCs plane and to have a comparably weak in-plane hexagonal symmetry.

Antiferromagnetic contribution: According to the phase diagram discussed in Sec.V.D an increase in the epitaxy temperature results in the formation of NCs with a higher degree of nitridation, for which AF coupling can prevail. In particular, $\gamma /-\mathrm{GaFe}_{3} \mathrm{~N}$ exhibits a weak AF coupling with a Curie-Weiss temperature of $-20 \mathrm{~K}$ (Houben et al. . 2009), whereas orthorhombic $\zeta-\mathrm{Fe}_{2} \mathrm{~N}$ is AF below $9 \mathrm{~K}$ (Hinomura and Nasu, 1996). Furthermore, it is also possible that wz-FeN NCs formed by chemical phase separation exhibit AF properties as, according to theoretical predictions presented in Sec.II.B, the coupling between Fe cation pairs is AF in GaN. These considerations led to the conclusion that a weakly saturating component of magnetization visible in Fig.41, rather than resulting from a Van Vleck paramagnetism could originate from antiferromagnetically ordered Fe-rich NCs (Navarro-Quezada et al. 2010).

The presence of various Fe-rich phases with peculiar magnetic properties has been proven, but now it is mandatory to explore the routes that can lead to a singlephase system, especially in the perspective of exploiting hybrid AF/FM systems for, e.g., AF spintronics (Shick et al. 2010).

\section{SPINODAL NANODECOMPOSITION IN (Ge,Mn}

\section{A. Mn dilution in germanium}

Almost all the theoretical predictions on the magnetic properties of magnetic semiconductors rely on the ideal dilution of magnetic TM atoms substituting the semiconducting host. $d$ levels of $\mathrm{Mn}$ atoms in a tetrahedral environment are split into two energy levels: $e_{g}$ (twice degenerated) and $t_{2 g}$ (3 times degenerated) levels. In germanium, $t_{2 g}$ orbitals strongly hybridize with $p$ orbitals in the germanium valence band forming bonding and antibonding states. The resulting magnetic moment per $\mathrm{Mn}$ atom is $3 \mu_{B}$ and two holes (when activated) are created in the germanium valence band. $A b$ initio calculations have further shown that exchange coupling between Mn atoms mediated by holes oscillates following the Rudermann-Kittel-Kasuya-Yoshida (RKKY) magnetic interaction (Continenza et al. 2006b. Zhao et al. , 2003). Among all TM atoms, $\mathrm{Mn}$ is the only one to provide both ferromagnetism and high localized spin moments in germanium. The Zener model complemented with mean field approximation further predicts $T_{\mathrm{C}}$ as high as $80 \mathrm{~K}$ in $\mathrm{Ge}_{0.975} \mathrm{Mn}_{0.025}$ with a hole density of $3.5 \times 10^{20} \mathrm{~cm}^{-3}$ (Dietl et al., 2000). However exchange coupling was shown to be highly anisotropic, i.e., to depend on the crystal orientation between two Mn atoms (Continenza et al., 2006b). Hence, for high Mn concentrations (up to a few percent), the relative position of Mn atoms in the germanium crystal lattice should be known in detail to understand magnetic properties. Finally, from band structure calculations, Mn dilution in germanium is more favorable than in silicon to induce half-metallic character $(100 \%$ spin polarization at the 
Fermi level) (Stroppa et al., 2003). To conclude about theoretical works, $(\mathrm{Ge}, \mathrm{Mn})$ might be a very promising candidate as a DMS in future spintronic devices compatible with mainstream silicon technology.

Surprisingly, only few works have been published on ferromagnetism in TM-doped germanium until 2002. The evidence of FM order in an epitaxial layer of Mndoped germanium was first reported in 2002 by Park (Park et al. 2002b). $\mathrm{Ge}_{1-x} \mathrm{Mn}_{x}(0.6<x<3.5 \%)$ films were grown by LT-MBE on Ge(001) and GaAs(001) substrates. They exhibit $p$-type doping with hole densities up to $10^{19}-10^{20} \mathrm{~cm}^{-3}$ and $T_{\mathrm{C}}$ increases linearly with $\mathrm{Mn}$ concentration from 25 up to $116 \mathrm{~K}$. Moreover they could demonstrate the interplay between band carriers and $\mathrm{Mn}$ spins by measuring AHE as shown in Fig.45(a). They could further modulate AHE by the application of a gate voltage as low as $0.5 \mathrm{~V}$. However the magnetic moment per Mn atom (1.4-1.9) $\mu_{B}$ ) was much less than the expected value for Mn substituting Ge $\left(3 \mu_{B}\right)$, and they reported the presence of small unidentified precipitates. Mn dilution in germanium is thus questionable.

\section{B. From Mn dilution to phase separation}

Considering the very low solubility of $\mathrm{Mn}$ in germanium $\left(10^{-6 \%}\right)$ (Woodbury and Tyler, 1955), out-ofequilibrium growth techniques are required to dope germanium films with a few percent of Mn. Indeed, according to the binary phase diagram (Massalski, 1990) and DFT calculations (Arras et al., 2011), the stable (Ge,Mn) alloy with the lowest $\mathrm{Mn}$ content is $\mathrm{Ge}_{8} \mathrm{Mn}_{11}$ and contains 57.9 at.\% of Mn. Since the 1980 s, seven stable $(\mathrm{Ge}, \mathrm{Mn})$ alloys have been synthesized: $\epsilon-\mathrm{GeMn}_{3.4}$ (Ohoyama et al. 1961), $\epsilon_{1}-\mathrm{GeMn}_{3.4}, \varsigma-\mathrm{Ge}_{2} \mathrm{Mn}_{5}$ (Ohba et al. 1987), $\kappa$ - $\mathrm{Ge}_{3} \mathrm{Mn}_{7}, \eta$ - $\mathrm{Ge}_{3} \mathrm{Mn}_{5}$ (Ohoyama, 1961), $\mathrm{GeMn}_{2}$ (Ellner, 1980) and $\mathrm{Ge}_{8} \mathrm{Mn}_{11}$ (Ohba et al. 1984 ). Although some of them are FM, they all exhibit a metallic character that makes them poor candidates for spin injection in nonmagnetic semiconductors due to conductivity mismatch (Fert and Jaffrès, 2001). Other (Ge,Mn) alloys could be prepared by melting Ge and Mn under very high pressure $(\simeq 4-6 \mathrm{GPa})$ by Takizawa. Metastable alloys such as $\mathrm{Ge}_{5} \mathrm{Mn}_{3}$ (Takizawa et al. , 1987), GeMn (Takizawa et al., 1988), $\mathrm{Ge}_{4} \mathrm{Mn}$ (Takizawa et al. 1990) or $\mathrm{GeMn}_{3}$ (Takizawa et al. 2002) could be obtained. All these $(\mathrm{Ge}, \mathrm{Mn})$ alloys are Ge rich and thus can exhibit a semiconducting character required for direct spin injection in semiconductors. We note in this context that pressure associated with the strain imposed by the Ge host can stabilize Ge rich $\mathrm{Ge}_{n} \mathrm{Mn}_{m}$ nanocrystals $(n>m)$ embedded in the Ge matrix. As discussed in Sec.VI.C, LT-MBE can serve to obtain such nanocomposites via spinodal nanodecomposition.

The very low solubility of Mn in Ge was further theoretically demonstrated using $a b$ initio calculations. For instance, in order to increase Mn concentration, mixing substitutional and interstitial $\mathrm{Mn}$ in germanium lowers the free energy of the system Arras et al. 2012 , Continenza et al. 2007). At last, except in the work by Zeng (Zeng et al. 2008), most groups have experimentally observed inhomogeneous Mn-doped germanium films. Inhomogeneities can be either secondary phase precipitates such as $\mathrm{Ge}_{3} \mathrm{Mn}_{5}$ clusters or Mn-rich nanostructures due to spinodal decomposition. Indeed spinodal decomposition leads to the formation of Mn-rich nanometer sized areas [either a metastable $(\mathrm{Ge}, \mathrm{Mn})$ alloy or Ge lattice with high Mn content] surrounded with an almost pure germanium matrix. In the next section, we thoroughly review the results obtained on the $(\mathrm{Ge}, \mathrm{Mn})$ material.

\section{Review of experimental results}

As already mentioned, in order to prevent the formation of stable metallic (Ge,Mn) phases, out-ofequilibrium growth techniques are required. In the following, we summarize Mn implantation in germanium and MBE growth of thin (Ge,Mn) films. Ottaviano et al. 2006 first performed $\mathrm{Mn}$ implantation in germanium (up to $4 \%$ ) at 240 and $270^{\circ} \mathrm{C}$ substrate temperatures. Before and after annealing (to improve the crystalline quality) Mn-rich precipitates are observed by TEM. These precipitates are amorphous before annealing and $\mathrm{Ge}_{3} \mathrm{Mn}_{5}$ clusters after annealing. In addition, x-ray absorption measurements showed that Mn-rich precipitates only form in the deeper part and substitutional Mn are detected close to the film surface (Ottaviano et al. , 2007). Ferromagnetic behavior was further observed by magnetooptical Kerr effect measurements up to $270 \mathrm{~K}$ (before annealing) and $255 \mathrm{~K}$ (after annealing). However the magnetic signal clearly arises from many different magnetic phases including $\mathrm{Ge}_{3} \mathrm{Mn}_{5}$. Passacantando et al. 2006 found similar results using different implantation doses in germanium wafers. At low implantation dose, Mn-rich precipitates are amorphous whereas they form $\mathrm{Ge}_{3} \mathrm{Mn}_{5}$ clusters at higher implantation doses as shown in Fig.45(b). The second technique widely used to grow Mn-doped Ge films is low-temperature MBE. Following the first results published by Park et al. 2002b, many groups have attempted to dilute large amounts of $\mathrm{Mn}$ in Ge in order to raise $T_{\mathrm{C}}$ up to RT. D'Orazio et al., 2004 investigated the magnetic and electrical properties of thin epitaxial $\mathrm{Ge}_{1-x} \mathrm{Mn}_{x}$ films $(0.027<x<0.044)$ exhibiting ferromagnetism up to $250 \mathrm{~K}$. Pinto et al., 2005 and Morresi et al., 2006 interpreted experimental observations in the framework of magnetic polaron percolation. However further structural characterizations showed that $(\mathrm{Ge}, \mathrm{Mn})$ epilayers contained $\mathrm{Ge}_{3} \mathrm{Mn}_{5}$ clusters surrounded with germanium containing almost $1.5 \%$ of substitutional paramagnetic Mn atoms. Similar results were also reported by others (Bihler et al. 2006; Padova et al., 2007, 2008). Finally, using high-resolution TEM and energy dispersive x-ray spectroscopy, Sugahara et al. 2005 showed the phase separation between Mn-rich amorphous clusters and a pure Ge matrix in thin epitaxial (Ge,Mn) films [see Fig.45(c)]. 


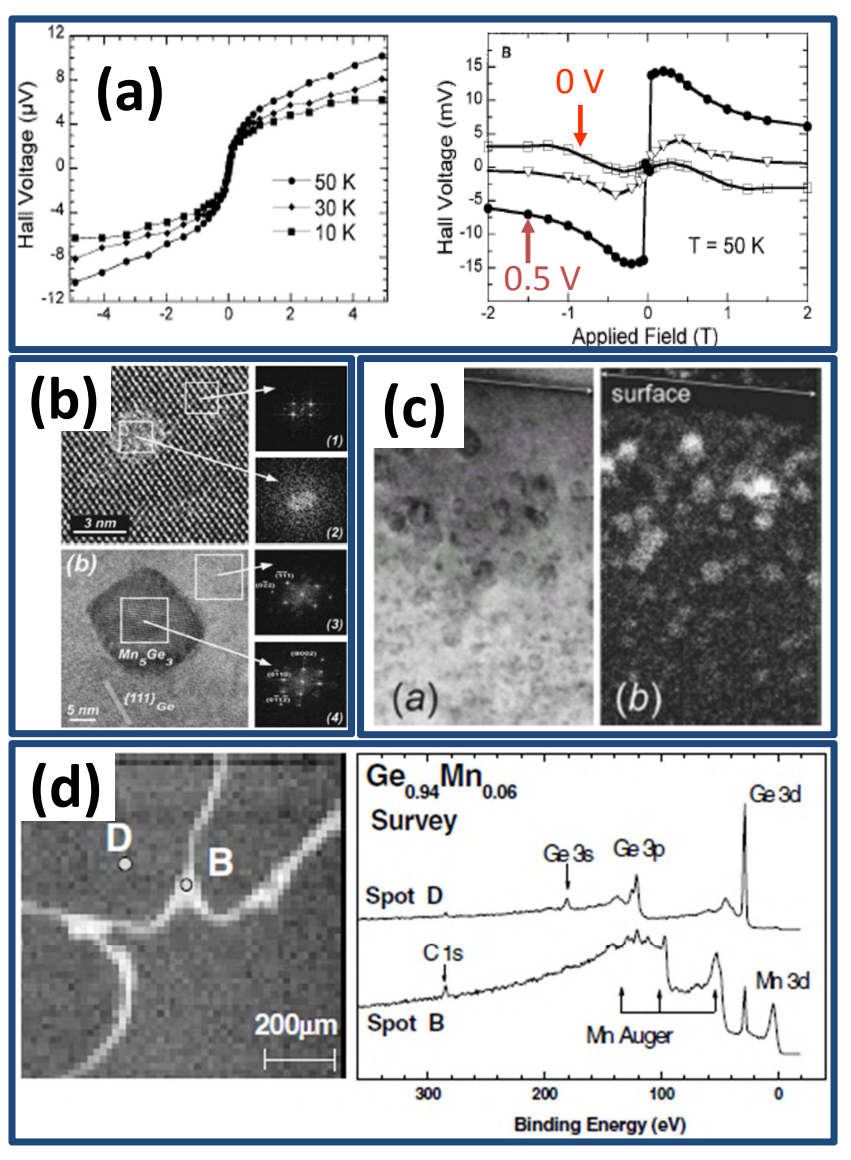

FIG. 45 (color online) Characterization of (Ge,Mn) samples obtained by various methods. (a) Anomalous Hall effect measured at 10,30 and $50 \mathrm{~K}$ in a $\mathrm{Mn}_{0.023} \mathrm{Ge}_{0.937}$ film grown by LT-MBE (left) and voltage control of AHE (right). From Park et al. 2002b. (b) HRTEM images of a Ge(100) wafer implanted at a dose of $1 \times 10^{16}$ (up) and $4 \times 10^{16}$ (down) $\mathrm{Mn}^{+}$ ions $/ \mathrm{cm}^{2}$. At low dose, Mn-rich areas are amorphous whereas at high dose, they form $\mathrm{Ge}_{3} \mathrm{Mn}_{5}$ clusters. From Passacantando et al. 2006 (c) TEM image of a GeMn film grown by LT-MBE showing amorphous $\mathrm{Ge}_{1-x} \mathrm{Mn}_{x}$ clusters (left) and corresponding energy dispersive x-ray spectroscopy image (right). Bright areas are Mn-rich. From Sugahara et al. 2005 (d) Scanning photoelectron microscopy image of a $\mathrm{Ge}_{0.94} \mathrm{Mn}_{0.06}$ single crystal (left) and the survey spectra obtained at a bright spot (B) and a dark spot (D) respectively (right). From Kang et al. 2005.

The second metallic (Ge,Mn) phase that usually forms during MBE growth is $\mathrm{Ge}_{8} \mathrm{Mn}_{11}$. This phase exhibits two magnetic transitions at $150 \mathrm{~K}(\mathrm{AF} / \mathrm{FM})$ and $T_{\mathrm{C}}=$ $285 \mathrm{~K}$ (FM/paramagnetic). $\mathrm{Ge}_{8} \mathrm{Mn}_{11}$ precipitates were observed by Park et al., 2001 in thin $\mathrm{Ge}_{1-x} \mathrm{Mn}_{x}$ films $(0<x<0.12)$ epitaxially grown between 250 and $300^{\circ} \mathrm{C}$. Using different methods to grow bulk single crystals, the same $\mathrm{Ge}_{8} \mathrm{Mn}_{11}$ phase could be detected by Cho et al. 2002, Kang et al., 2005 and Biegger et al., 2007 performing chemical analysis at the micrometer scale, as shown in Fig. 45(d). More recently, by investigating Mn-doped (up to $4 \%$ ) Ge films epitaxially grown on Ge(001) using various TEM techniques, Wang et al., 2008 could find the coexistence of $\mathrm{Ge}_{8} \mathrm{Mn}_{11}$ and $\mathrm{Ge}_{2} \mathrm{Mn}_{5}$ clusters. Moreover, by well adjusting growth parameters, these crystalline precipitates could be replaced by undefined Mn-rich nanostructures.

Since 2005, many groups reported the absence of any secondary phase precipitation in $(\mathrm{Ge}, \mathrm{Mn})$ films epitaxially grown at low temperature $\left(<100^{\circ} \mathrm{C}\right)$. However, due to the very low solubility of $\mathrm{Mn}$ in $\mathrm{Ge}$, the formation of Mn-rich nanostructures seems unavoidable mostly as a result of spinodal nanodecomposition. These nanostructures are so hard to detect that only highly sensitive techniques such as TEM with nanoscale chemical analysis, 3DAP or XRD/XAS using synchrotron radiation can be used. From the low-temperature MBE growth of $\mathrm{Ge}_{0.94} \mathrm{Mn}_{0.06}$ films, Sugahara et al., 2005 found elongated Mn-rich amorphous precipitates surrounded with pure germanium. These precipitates exhibit ferromagnetism up to $100 \mathrm{~K}$ as given by MCD measurements. Their diameter is close to $5 \mathrm{~nm}$ and the $\mathrm{Mn}$ content is between 10 and $20 \%$. Using almost the same growth conditions, Ahlers et al., 2006; Bougeard et al., 2006, 2009 observed the formation of Mn-rich cubic clusters coherently strained on the Ge matrix in addition to the precipitation of $\mathrm{Ge}_{3} \mathrm{Mn}_{5}$ clusters. Assuming zero Mn content in the Ge matrix, the maximum Mn content in the clusters is close to $15 \%$. Their diameter is slightly less than $5 \mathrm{~nm}$. Moreover they exhibit superparamagnetic behavior and a $T_{\mathrm{C}}$ value close to $200 \mathrm{~K}$. Still using low-temperature MBE growth and according to magnetic measurements only, Jaeger et al. 2006 concluded that $\mathrm{Ge}_{1-x} \mathrm{Mn}_{x}$ films $(x=0.04$ and 0.2$)$ contain two cluster populations (undefined Mn-rich precipitates and $\mathrm{Ge}_{3} \mathrm{Mn}_{5}$ clusters) along with diluted $\mathrm{Mn}$ atoms in the germanium matrix. The overall population of Mn-rich precipitates behaves as a spin glass. The freezing temperatures are 12 and $15 \mathrm{~K}$ in $\mathrm{Ge}_{0.96} \mathrm{Mn}_{0.04}$ and $\mathrm{Ge}_{0.8} \mathrm{Mn}_{0.2}$ films, respectively. Finally Li et al. 2007 also found elongated Mn-rich precipitates but coherently strained on the surrounding Ge matrix. In as-grown $\mathrm{Ge}_{0.95} \mathrm{Mn}_{0.05}$ samples, these precipitates show ferromagnetism at low temperature: remanence vanishes above $12 \mathrm{~K}$. Post-growth annealing at $200^{\circ} \mathrm{C}$ leads to a rather substantial improvement in magnetic and electrical properties by converting interstitial Mn into substitutional Mn. They exhibit remanence up to $125 \mathrm{~K}$, the magnetic moment per $\mathrm{Mn}$ atom reaches $1.5 \mu_{B}$ instead of $1.0 \mu_{B}$ in as-grown samples. Moreover, annealing triggers strong positive MR and AHE in (Ge,Mn) films.

To summarize, the key issue of $(\mathrm{Ge}, \mathrm{Mn})$ material is Mn-dilution: the very low solubility of Mn in Ge always results in Mn segregation and in the formation of Mnrich precipitates. Low-temperature growth techniques favor the formation of metastable (Ge,Mn) phases. As a consequence, slight differences in growth parameters can result in much different magnetic and electrical properties. In the following sections, we show how to control 
spinodal nanodecomposition in (Ge,Mn). The structure, magnetic, and electrical properties of (Ge,Mn) films with spinodal nanodecomposition are reviewed.

\section{Growth and structure of thin (Ge,Mn) films with spinodal decomposition}

\section{Sample preparation}

Growth was performed using solid sources MBE by codepositing Ge and Mn evaporated from standard Knudsen effusion cells (Devillers et al., 2007a, 2006, 2007b; Jamet et al. 2006). The deposition rate was kept constant and quite low $\left(\simeq 0.2 \AA_{\mathrm{s}}^{-1}\right)$. Germanium substrates were epiready $\mathrm{Ge}(001)$ wafers with a residual $n$-type doping and resistivity of $10^{15} \mathrm{~cm}^{-3}$ and $5 \Omega \mathrm{cm}$, respectively. After thermal desorption of the surface oxide, a 40 nm-thick Ge buffer layer was grown at $250^{\circ} \mathrm{C}$, resulting in a $2 \times 1$ surface reconstruction as observed by RHEED (see Fig.46). Next, 80 nm-thick $\mathrm{Ge}_{1-x} \mathrm{Mn}_{x}$ films were subsequently grown at low substrate temperature (between $T_{g}=80^{\circ} \mathrm{C}$ and $T_{g}=200^{\circ} \mathrm{C}$ ). Mn content has been determined by x-ray fluorescence measurements performed on thick samples $(\simeq 1 \mu \mathrm{m}$ thick) and complementary Rutherford back scattering (RBS) on thin $\mathrm{Ge}_{1-x} \mathrm{Mn}_{x}$ films grown on silicon. Mn concentrations range from 1 to $11 \% \mathrm{Mn}$.
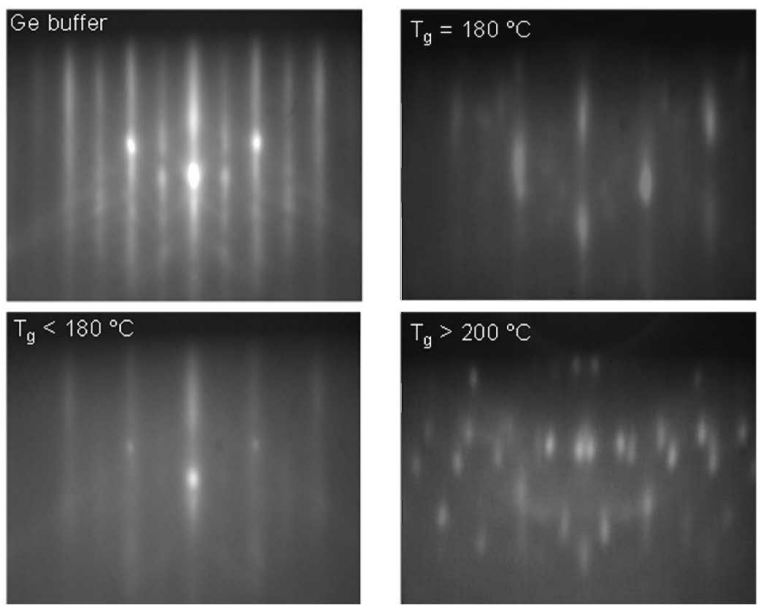

FIG. 46 RHEED patterns recorded for different growth temperatures of $\mathrm{Ge}_{1-x} \mathrm{Mn}_{x}$. Adapted from Devillers et al. 2007a.

For $\mathrm{Ge}_{1-x} \mathrm{Mn}_{x}$ films grown at substrate temperatures below $180^{\circ} \mathrm{C}$, after the first monolayer (ML) deposition, the $2 \times 1$ surface reconstruction almost totally disappears. After depositing a few MLs (corresponding almost to $5 \mathrm{~nm}$ ), a slightly diffuse $1 \times 1$ streaky RHEED pattern and a very weak $2 \times 1$ reconstruction (Fig. 46) indicate the predominantly $2 \mathrm{D}$ growth of a single crystalline film exhibiting the same lattice parameter as the Ge buffer layer. Increasing the layer thickness leads to an amplification of the surface roughness which is expected for the low-temperature epitaxial growth of germanium Chason et al. 1989; Venkatasubramanian and Dorsey, 1993). For growth temperatures above $180^{\circ} \mathrm{C}$ additional spots appear in the RHEED pattern during the $\mathrm{Ge}_{1-x} \mathrm{Mn}_{x}$ growth (Fig.46). These spots correspond to the formation of very small $\mathrm{Ge}_{3} \mathrm{Mn}_{5}$ crystallites.

\section{Morphology of (Ge,Mn) films}

From TEM images, vertical elongated nanostructures, i.e., nanocolumns were observed as shown in Fig.47(a). These observations are in very good agreement with the theoretical predictions of Sec. II] The formation of the konbu phase as a consequence of $2 \mathrm{D}$ spinodal decomposition in $(\mathrm{Ge}, \mathrm{Mn})$ is clearly demonstrated here as well as in $(\mathrm{Zn}, \mathrm{Cr}) \mathrm{Te}$ in Sec. VIII. Similar elongated nanostructures were also obtained in (Ge,Mn) by several groups in comparable growth conditions [Fig. $47(\mathrm{~b}-\mathrm{e})]$. Nanocolumns span the entire $\mathrm{Ge}_{1-x} \mathrm{Mn}_{x}$ film thickness. Whatever the growth temperature and $\mathrm{Mn}$ concentration (except $0.1 \%), \mathrm{Ge}_{1-x} \mathrm{Mn}_{x}$ films always exhibit the presence of nanocolumns with their axis along the growth direction [001]. Depending on growth conditions, the average columns diameter and density range between 2 and $7 \mathrm{~nm}$ and between 10000 and $40000 \mu \mathrm{m}^{-2}$, respectively.

Further evidence of $2 \mathrm{D}$ spinodal nanodecomposition was provided by analyzing the periodic structures of the nanocolumns (Hai et al., 2011; Yada et al., 2011). Figure 48(a) presents a plan view TEM image showing that the nanocolumns are uniform in diameter $(\sim 3 \mathrm{~nm})$, and form either rectangular or triangular lattice structures, as indicted by thin dashed lines in Fig. 48(a). According to the Fourier transform of the TEM image, depicted in Fig. 48(b), the average distance between the nanocolumns is $\sim 9 \mathrm{~nm}$.

The morphology of $\mathrm{Ge}_{1-x} \mathrm{Mn}_{x}$ films was further investigated using grazing incidence small angle x-ray scattering (GISAXS) from synchrotron radiation and atomic force microscopy (AFM), as depicted in Fig. 49

As shown in Fig. 49.(a), in addition to the specular reflection peak in the GISAXS spectrum, two pairs of correlation peaks were observed. The first one at large $q_{x}$ value corresponds to a correlation length of $\simeq 10 \mathrm{~nm}$ which is the average distance between nanocolumns. The oscillations along $q_{z}$ are thickness oscillations and stand for the finite thickness of the $\mathrm{Ge}_{1-x} \mathrm{Mn}_{x}$ film. It demonstrates that nanocolumns span the entire film thickness. Furthermore there is a sizable contrast of electronic density between nanocolumns and the surrounding matrix. At low $q_{x}$ value, a second pair of correlation peaks corresponds to a distance of almost $50 \mathrm{~nm}$. Moreover these peaks are much more elongated along $q_{z}$ which is characteristic of a surface effect. Indeed, correlations are no more related to the presence of nanocolumns but to the surface roughness, as shown in Fig. 49(b). In order to investigate the columns chemical composition, electron 


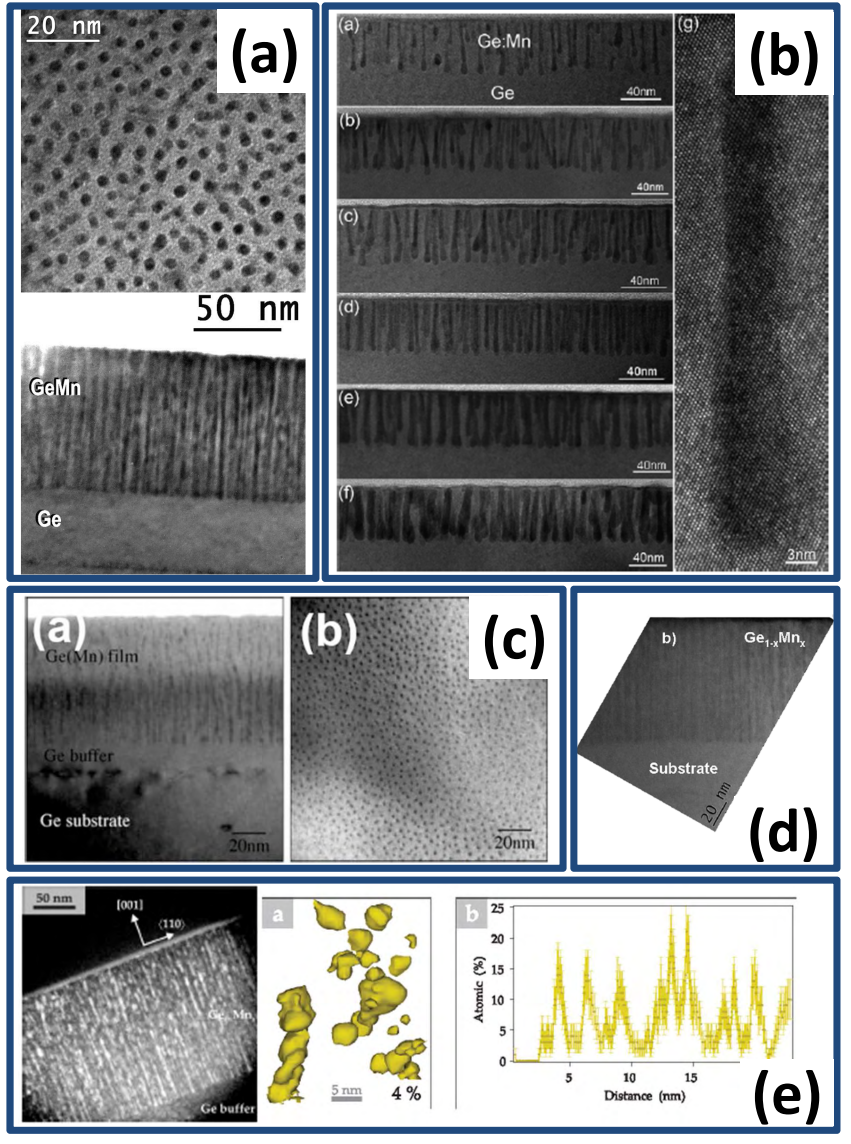

FIG. 47 (Color online) Characterization of $\mathrm{Ge}_{1-x} \mathrm{Mn}_{x}$ thin films grown by LT-MBE. (a) TEM plane view (up) and cross section (down) images of $\mathrm{Ge}_{0.9} \mathrm{Mn}_{0.1}$ containing Mnrich nanocolumns. From Devillers et al., 2007a. (b) TEM cross section images of films with $x=2.5 \%, 4,4.5,5.5,7$, and $12 \%$ from top to bottom. The morphology of Mn-rich nanocolumns evolves from tadpolelike to cylinders when increasing Mn content. From Wang et al. 2010. (c) and (d) TEM images of $\mathrm{Ge}_{0.95} \mathrm{Mn}_{0.05}$ [from Li et al. , 2007] and $\mathrm{Ge}_{0.94} \mathrm{Mn}_{0.06}$ [from Le et al., 2011], respectively, showing the presence of nanocolumns. (e) Vertical self-assembly of roughly spherical Mn-rich clusters: TEM cross section image in dark field with $x=7.3 \%$ (left) and atom probe tomography data for $x=2 \%$ (right). From Bougeard et al. 2009 .

energy loss spectroscopy (EELS) has been performed in cross section and plane view. The corresponding EFTEM images close to the $\mathrm{Mn} L_{2,3}$ edge are displayed in Fig. 50 along with the corresponding Mn chemical profiles.

From EFTEM images, one can conclude that nanocolumns are Mn-rich and surrounded with an almost pure Ge matrix. The Mn signal in the Ge matrix is indeed below the resolution limit of EELS spectroscopy estimated to be around $1 \%$. The most appropriate technique to estimate with more accuracy the Mn content in the Ge matrix is 3DAP. It leads to an average Mn con- (a)
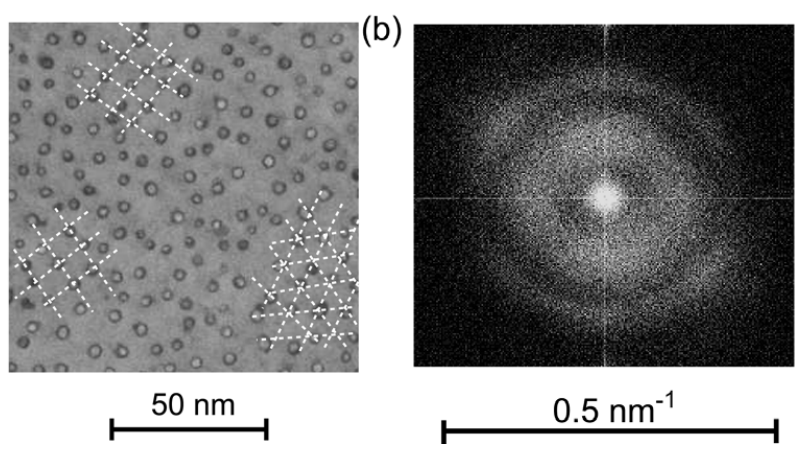

FIG. 48 (a) Plan view TEM image of Mn-rich nanocolumns embedded in a Ge matrix fabricated by MBE of a $30 \mathrm{~nm}-$ thick $\mathrm{Ge}_{0.94} \mathrm{Mn}_{0.06}$ film onto a $\mathrm{Ge}(001)$ substrate at $100^{\circ} \mathrm{C}$ with a growth rate of $150 \mathrm{~nm} / \mathrm{h}$. (b) Fourier transform of the contrast of a $0.36 \times 0.36 \mu \mathrm{m}^{2}$ TEM image. Ring structures up to second harmonic are clearly seen, indicating strong $2 \mathrm{D}$ spinodal decomposition. Adapted from Hai et al., 2011.
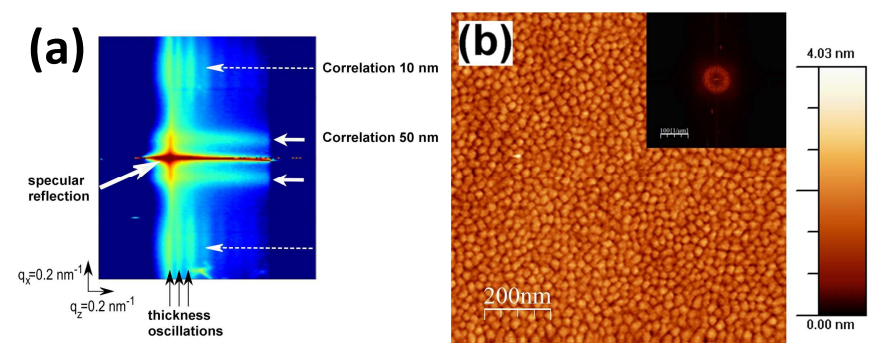

FIG. 49 (Color online) Characterization of a $\mathrm{Ge}_{0.93} \mathrm{Mn}_{0.07}$ film grown at $150^{\circ} \mathrm{C}$. (a) GISAXS spectrum; the nanocolumns diameter is $5 \mathrm{~nm}$ and their density $8100 \mu \mathrm{m}^{-2}$. Adapted from Tardif et al. 2010b. (b) Corresponding AFM image of the film surface. The maximum roughness amplitude is of the order of $1 \mathrm{~nm}$ and corresponds to the formation of small islands at the film surface. The fast Fourier transform (inset) of this picture exhibits the presence of a correlation ring between surface islands of the order of $30 \mathrm{~nm}$. From Devillers, 2008.

centration inside the Ge matrix below $0.05 \%$ ((Mouton et al. 2012)). As a consequence, the Mn concentration in the nanocolumns shown in Fig. 50 is close to $30 \%$. Hence the composition of nanocolumns is close to $\mathrm{Ge}_{2} \mathrm{Mn}$. Depending on the growth conditions, nanocolumns with Mn concentrations between 5 and $50 \%$ could be grown, all of them exhibiting FM properties (Devillers et al., 2007a).

\section{Lateral and vertical control of nanocolumns}

In order to achieve lateral control of nanocolumns (diameter and density), the growth temperature and Mn concentration were varied to study their respective influence. For this purpose, two series of $\mathrm{Ge}_{1-x} \mathrm{Mn}_{x}$ films were grown at two different growth temperatures: $T_{g}=$ 100 and $T_{g}=150^{\circ} \mathrm{C}$. In each case, the Mn concentration 

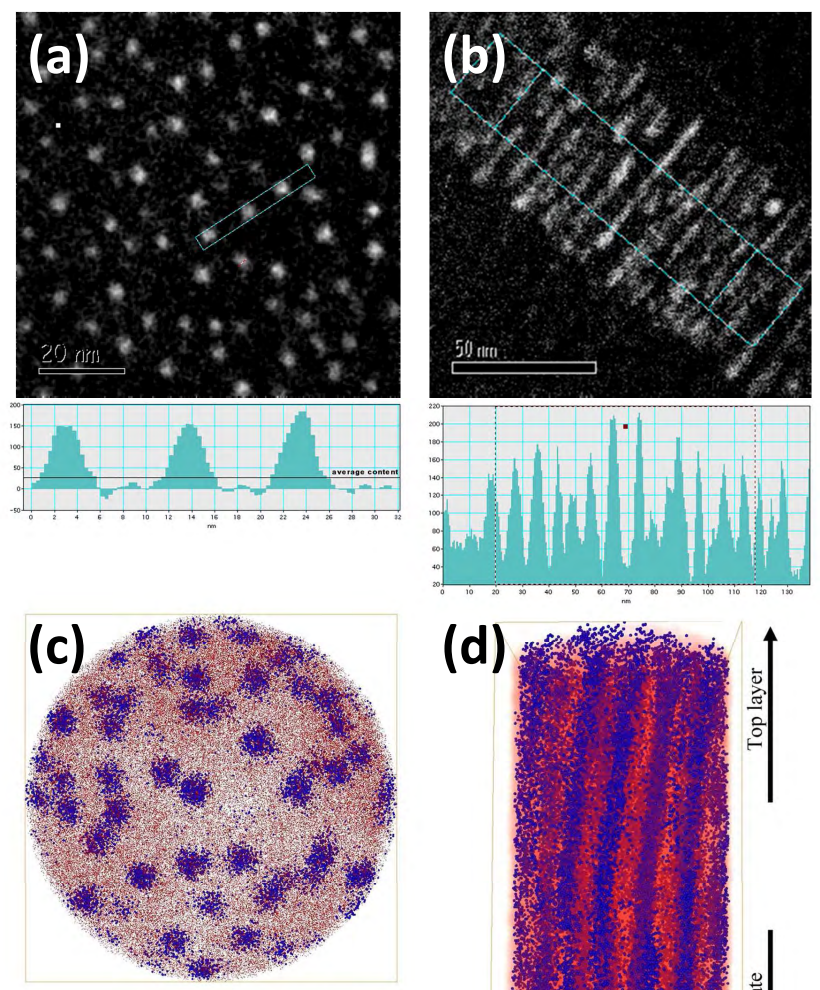

$\left(33 \times 33 \times 10 \mathrm{~nm}^{3}\right)$

- Mn atoms

Ge matrix

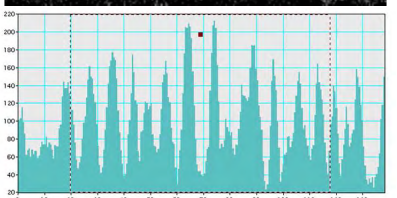

(d)
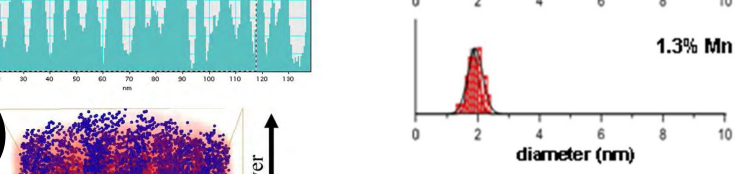

11.3\% $\mathrm{Mn}$

(a)
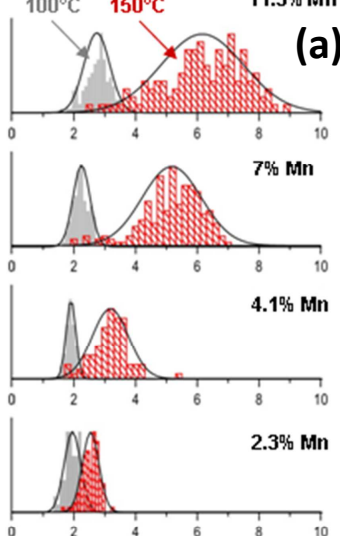
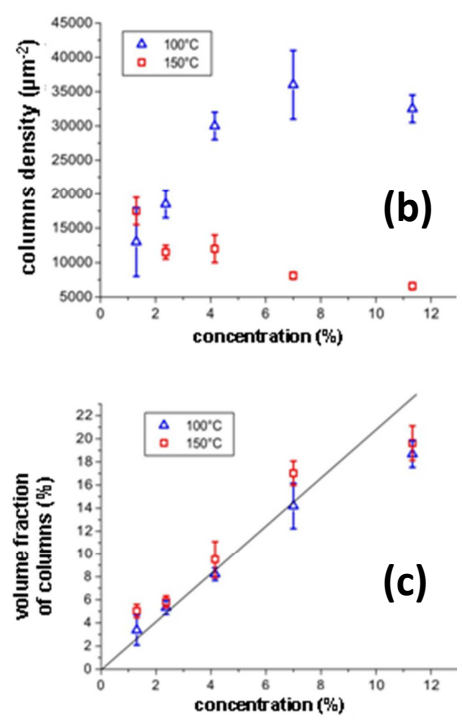

FIG. 51 (Color online) Lateral control of nanocolumns. (a) Numbers of $(\mathrm{Ge}, \mathrm{Mn})$ nanocolums with a given radius (size distribution) extracted from plane view TEM images for samples with various average $\mathrm{Mn}$ concentrations $x(1.3 \%, 2.3 \%$, $4.1 \%, 7 \%$, and $11.3 \%$ ) for two growth temperatures $100^{\circ} \mathrm{C}$ (filled bars) and $150^{\circ} \mathrm{C}$ (dashed bars). (b) Density (in $\mu \mathrm{m}^{-2}$ ) and (c) volume fraction of nanocolumns as a function of $x$ for these two growth temperatures (open triangles and open squares, respectively). From Devillers et al. $2007 \mathrm{a}$.

ever, according to Fig.51(a), their average diameter increases significantly and size distributions become much broader. For the highest Mn concentration (11.3\%) very small columns with a diameter of $2.5 \mathrm{~nm}$ coexist with very large columns with a diameter of $9 \mathrm{~nm}$. Therefore increasing $\mathrm{Mn}$ concentration in samples grown at $150^{\circ} \mathrm{C}$ allows to control the columns' diameter while keeping their density constant around $10000 \mu \mathrm{m}^{-2}$. To summarize, by combining two growth parameters (temperature and $\mathrm{Mn}$ concentration), it is possible to independently control the diameter and density of nanocolumns. This ability is of great importance from a fundamental point of view to characterize nanocolumns but also for potential applications in spintronic devices. In Fig.51(c), the volume fraction occupied by the columns in the film is reported as a function of Mn concentration showing a clear linear dependence for Mn contents up to almost $7 \%$. The nonlinear behavior above $7 \%$ can indicate that the mechanism of $\mathrm{Mn}$ incorporation is different in this concentration range, leading to an increase of the $\mathrm{Mn}$ concentration in the columns and/or in the matrix.

Considering the vertical geometry of nanocolumns and the Mn concentration contrast between the columns and the Ge matrix, vertical transport will mainly take place through nanocolumns. Since nanocolumns are FM (see Sec.VI.D), they could be used as conduction channels for spin injection in nonmagnetic semiconductors such as

$\mathrm{Si}$ and Ge. The vertical control of nanocolumns is thus at $150^{\circ} \mathrm{C}$ from 1.3 to $11.3 \%$ leads to a slight decrease of the columns density as shown in Fig. 51(b). How- 

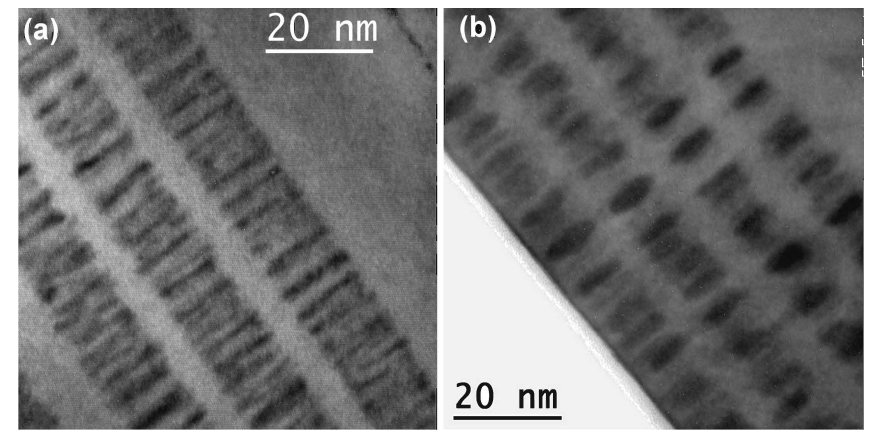

FIG. 52 (Color online) HRTEM images of (GeMn/Ge) 4 superlattices observed along the [110] crystal axis. From Devillers, 2008. In (a), no vertical correlation is observed whereas in (b) the vertical positions are clearly correlated and columns well aligned. In both cases, the germanium spacer is $5 \mathrm{~nm}$ thick.

an important issue from a fundamental point of view as well as for potential applications in spintronics. For this purpose, (GeMn/Ge) superlattices have been grown using different growth conditions. The $(\mathrm{GeMn} / \mathrm{Ge})_{4}$ superlattice in Fig.52 (a) was grown in standard conditions $\left(0.2 \AA / \mathrm{s}, 6 \%\right.$ of $\left.\mathrm{Mn}, T_{g}=100^{\circ} \mathrm{C}\right)$ leading to the formation of small nanocolumns with no vertical selforganization. The superlattice in Fig. 52(b) was grown at higher temperature $\left(140^{\circ} \mathrm{C}\right)$ using a lower growth rate $(0.13 \AA / \mathrm{s})$ in order to favor the surface diffusion of $\mathrm{Mn}$. Moreover the $\mathrm{Mn}$ content was increased up to $10 \%$ to grow larger columns. Assuming that the lattice parameters within the columns and the Ge matrix are different, larger columns lead to an enhanced residual strain field which favors the nucleation of nanocolumns on top of each other as in quantum dot superlattices (Tersoff et al. 1996). By this method, the vertical self-organization of nanocolumns could be achieved (Wang et al., 2011). Since the interface between nanocolumns and Ge spacer are quite abrupt, the diffusion of Mn along the growth direction is limited and then restricted to the top surface layer as discussed next.

\section{Two-dimensional spinodal decomposition}

In order to understand the growth mechanism of nanocolumns, the first growth stage of $(\mathrm{Ge}, \mathrm{Mn})$ films on $\mathrm{Ge}(001)$ were investigated. For this purpose, STM images have been recorded after depositing $0.44 \AA$ of $\mathrm{Mn}$ on $\mathrm{Ge}(001)$ at $80^{\circ} \mathrm{C}$ [Fig.53(a)].

The images show the formation of self-assembled nanoislands. Their size and density are further comparable to the size and density of nanocolumns in as-grown (Ge,Mn) films [Fig.53(b)]. Therefore, nanoislands are most probably Mn-rich nuclei at the origin of the formation of nanocolumns. Accordingly, this observation implies that the columns size and density are fully determined at the first growth stage. This growth mode

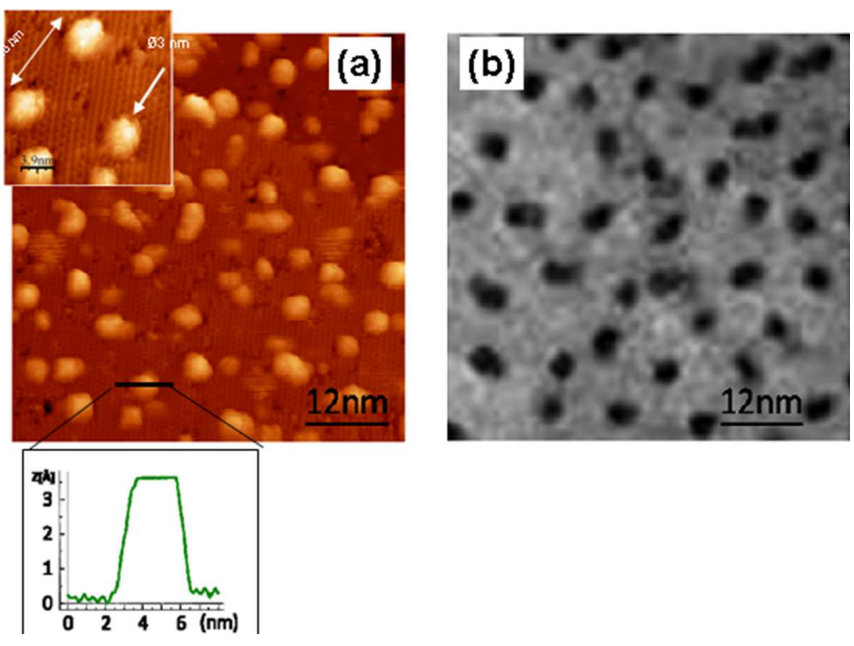

FIG. 53 (Color online) Formation of nanocolums from selfassembled nanoislands. (a) STM image of a germanium surface after depositing $0.44 \AA$ of $\mathrm{Mn}$ at $80^{\circ} \mathrm{C}$ (from Lethanh, 2006). The inset shows the diameter of self-assembled islands $(\simeq 3 \mathrm{~nm})$ and the distance between nearest-neighbors $(\simeq 10 \mathrm{~nm})$. The reported $z$-profile gives an estimation of their height: $3.5 \AA$. (b) Plan view TEM image of a $80 \mathrm{~nm}$ thick $\mathrm{Ge}_{0.94} \mathrm{Mn}_{0.06}$ film grown at $130^{\circ} \mathrm{C}$. Adapted from Jamet et al. 2006

leading to the formation of the konbu phase is summarized in Sec. III. It results from 2D spinodal decomposition due to Mn-Mn chemical pair attraction followed by layerby-layer growth (Fukushima et al., 2006b; Zheng et al. 2010). The expected scenario for the growth of Mn-rich nanocolumns is illustrated in Fig.54.

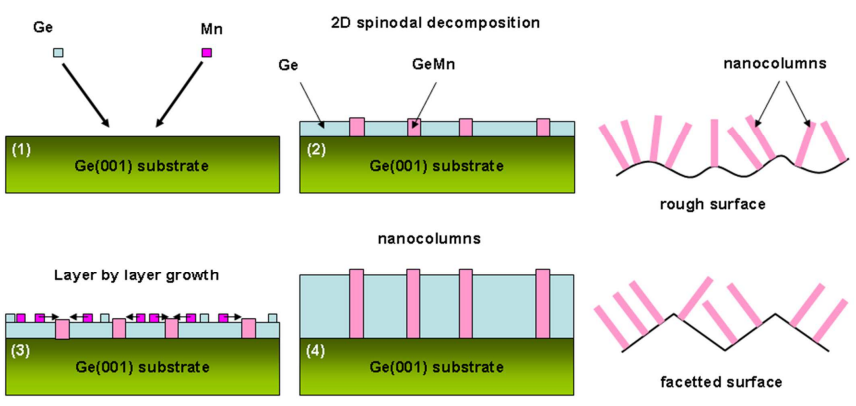

FIG. 54 (Color online) Nanocolumn growth and geometry. Left: four-steps scenario for the growth of Mn-rich nanocolumns in germanium. (1) Ge and Mn atoms are codeposited by LT-MBE on the germanium surface. (2) Because of strong Mn-Mn pair attraction, a two-dimensional spinodal decomposition takes place at the first growth stage setting the size and density of nanocolumns. (3) Layer-by-layer growth with surface diffusion of $\mathrm{Mn}$ atoms. Because of $\mathrm{Mn}-\mathrm{Mn}$ pair attraction, $\mathrm{Mn}$ atoms aggregate on top of $\mathrm{Mn}$-rich areas. (4) Mn-rich nanocolumns perpendicular to the initial surface are obtained. Right: expected columns geometries for a rough and facetted initial surface. From Jamet, 2010. 
As a consequence, nanocolumns will always grow perpendicular to the initial surface. Therefore, they will exactly follow the initial surface roughness or facets (Fig.54). In order to support this conclusion, thin $(\mathrm{Ge}, \mathrm{Mn})$ films were grown in standard growth conditions on a rough $\mathrm{GaAs}(001)$ surface as well as on a facetted $\mathrm{Ge}$ surface (Yu et al. 2010, 2011).
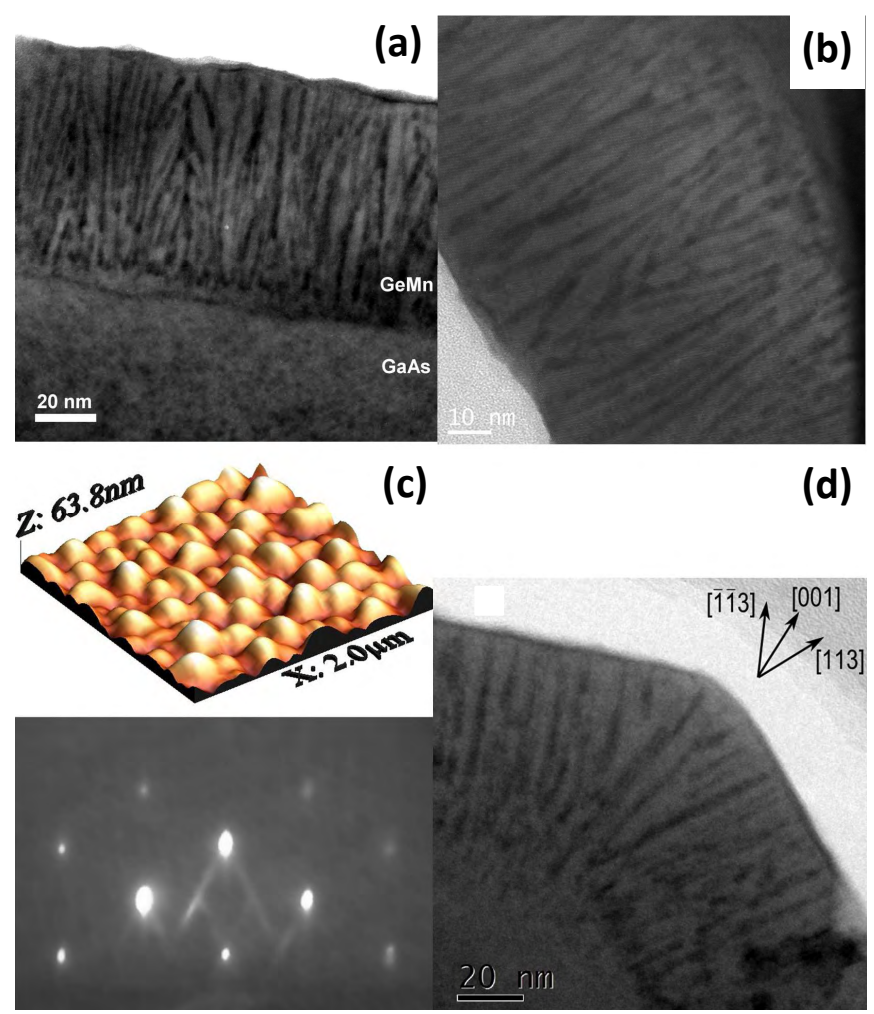

FIG. 55 (Color online) (a) Low and (b) HRTEM cross section images of a $80 \mathrm{~nm}$-thick $\mathrm{Ge}_{0.9} \mathrm{Mn}_{0.1}$ film grown at $100^{\circ} \mathrm{C}$ on $\operatorname{GaAs}(001)$. Before the growth, the native oxide layer was first thermally removed from the $\mathrm{GaAs}(001)$ substrate at $600^{\circ} \mathrm{C}$ leading to a rough Ga-rich surface. (c) AFM image and RHEED pattern of a facetted Ge surface obtained after anisotropic chemical etching revealing $\{113\}$ facets. (d) TEM cross section image of a $80 \mathrm{~nm}$-thick $\mathrm{Ge}_{0.94} \mathrm{Mn}_{0.06}$ film grown at $100^{\circ} \mathrm{C}$ on the facetted Ge surface. From Yu et al. 2010

The resulting (Ge,Mn) films were then observed by TEM in cross section (Fig.55). On $\operatorname{GaAs}(001)$, Mn-rich nanocolumns are bent following the initial surface roughness. On the facetted Ge surface, nanocolumns have their axis perpendicular to the $\{113\}$ facets (Yu et al. 2010$)$. Hence 2D spinodal decomposition and further layer-bylayer growth are most probably the mechanisms responsible for the formation of Mn-rich nanocolumns.

\section{Crystal structure of nanocolumns}

The crystalline structure of (Ge,Mn) films was investigated using high-resolution TEM observations as well as XRD and XAS. High resolution TEM observations in cross section show that $\mathrm{Ge}_{1-x} \mathrm{Mn}_{x}$ films grown below $180^{\circ} \mathrm{C}$ are single crystalline in epitaxial relationship with the substrate [Fig.556(a)]. Furthermore nanocolumns can hardly be distinguished. The interface between the $\mathrm{Ge}$ buffer layer and the $\mathrm{Ge}_{1-x} \mathrm{Mn}_{x}$ film is flat and no defect propagates from the interface into the film. Diffraction scans performed in $\theta / 2 \theta$ mode were acquired on a high-resolution diffractometer using synchrotron radiation. The scans reveal only the (004) Bragg peak of the germanium crystal, confirming the good epitaxial relationship between the layer and the substrate, and the absence of secondary phases to a good accuracy proven by a high ratio of the main peak intensity to noise, of the order of $10^{7}$ [inset in Fig. 56.(a)].

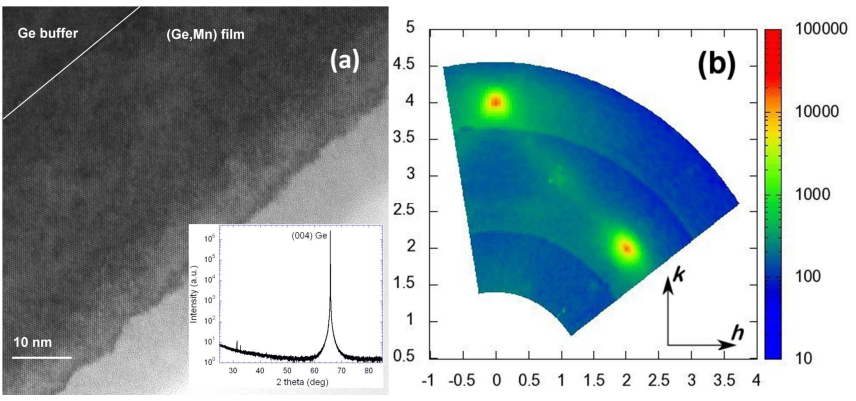

FIG. 56 (Color online) (a) HRTEM image of a $80 \mathrm{~nm}$ thick $\mathrm{Ge}_{0.94} \mathrm{Mn}_{0.06}$ sample grown at $130^{\circ} \mathrm{C}$. Inset: XRD showing the single (004) Bragg peak of pure Ge. (b) $(h, k, 0)$ map of the reciprocal space performed on a $\mathrm{Ge}_{0.94} \mathrm{Mn}_{0.06}$ film grown at $150^{\circ} \mathrm{C}$. Adapted from Tardif et al. 2010b.

In order to get further insight into the crystalline structure of nanocolumns, grazing incidence SXRD was studied. The reciprocal space of a $\mathrm{Ge}_{0.94} \mathrm{Mn}_{0.06}$ film grown at $150^{\circ} \mathrm{C}$ [Fig. 56(b)] was mapped. In addition to the (400) and (220) Bragg peaks of germanium, two additional features were found: a broad (130) peak (which is forbidden in the diamond lattice) and a diffuse line connecting (400) and (220) Bragg peaks. At this stage, none of them could be related to the crystalline structure of nanocolumns (Tardif et al. 2010b). (220) and (400) Bragg peaks further exhibit correlation rings related to anisotropic elastic strain in the germanium matrix between nanocolumns (Tardif et al. 2010b). Since the internal crystalline structure of nanocolumns could not be resolved using XRD, three conclusions can be drawn: (i) nanocolumns exhibit the same diamond lattice as germanium with high Mn content, (ii) they are made of a metastable $(\mathrm{Ge}, \mathrm{Mn})$ phase showing a diffraction pattern similar to that of pure germanium and (iii) they are amorphous. In the following, the decisive role of the growth temperature on the columns crystalline structure is discussed.

High-resolution TEM observations in plan view clearly demonstrate an evolution of the columns structure with growth temperature (Fig.57). For $T_{g}=100^{\circ} \mathrm{C}$, small 

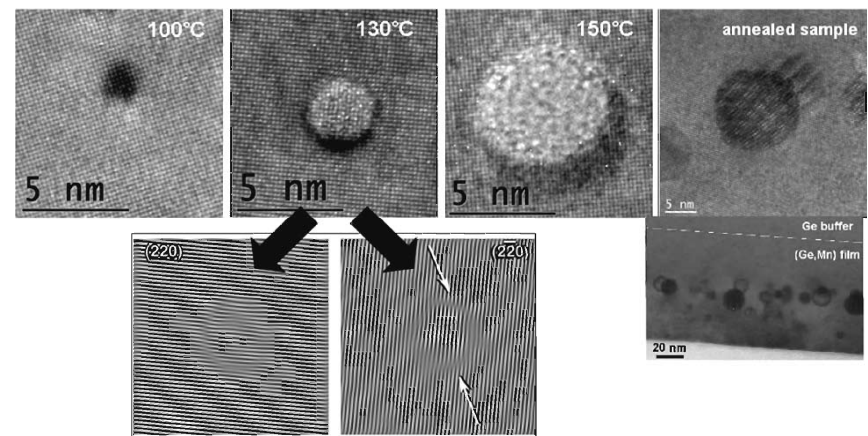

FIG. 57 (Color online) Crystalline structure of nanocolumns as a function of growth temperature. From left to right: $\mathrm{Ge}_{0.99} \mathrm{Mn}_{0.01}$ film grown at $100^{\circ} \mathrm{C} ; \mathrm{Ge}_{0.94} \mathrm{Mn}_{0.06}$ film grown at $130^{\circ} \mathrm{C} ; \mathrm{Ge}_{0.887} \mathrm{Mn}_{0.113}$ film grown at $150^{\circ} \mathrm{C} ; \mathrm{Ge}_{0.94} \mathrm{Mn}_{0.06}$ film grown at $130^{\circ} \mathrm{C}$ and annealed at $650^{\circ} \mathrm{C}$ for $15 \mathrm{~min}$. Adapted from Jamet et al. 2006 and Devillers et al. 2007a.

nanocolumns are fully strained on the surrounding Ge matrix and clearly exhibit a perfect cubic crystal. For $T_{g}=130^{\circ} \mathrm{C}$, crystalline columns were still observed. However, performing (220) and (2产) Bragg filtering of diffraction spots, a pair of dislocations at the interface between the column and the Ge matrix is clearly visible. This leads to a lattice expansion of almost $4 \%$ in the column. Nanocolumns are thus only partially strained. For $T_{g}=150^{\circ} \mathrm{C}$, nanocolumns are amorphous. Finally, for samples grown at $T_{g}>180^{\circ} \mathrm{C}$ or annealed at high temperature, nanocolumns have collapsed into stable $\mathrm{Ge}_{3} \mathrm{Mn}_{5}$ clusters. For annealed samples, these clusters are further well buried inside the germanium film away from the surface.

Since magnetic properties highly depend on the relative position of $\mathrm{Mn}$ atoms in the nanocolumns, the local chemical environment of Mn atoms was probed by EXAFS measurements (Rovezzi et al. 2008). By fitting EXAFS oscillations, the chemical environment of Mn atoms in the nanocolumns was found very similar to that of a $\mathrm{Mn}$ atom in an elementary tetrahedron of the $\mathrm{Ge}_{3} \mathrm{Mn}_{5}$ unit cell. This elementary tetrahedron corresponds to the building block of nanocolumns. However, at this stage, the structure of crystalline columns grown at low temperature still remains unknown. Nevertheless, a possible phase which exhibits a crystalline structure and composition compatible with the experimental findings was proposed by Arras et al., 2012. This phase is called the $\alpha$ phase and corresponds to a new class of materials based on the insertion of $\mathrm{Mn}$ atoms into a simple cubic crystal of Ge. Arras et al., 2012 showed that the incorporation of this phase (with a Mn content ranging from 10 to 50\%) in the form of nanocolumns into the pure Ge matrix is energetically favorable.

\section{Magnetic properties}

In this section, the magnetic properties of four different $\mathrm{Ge}_{1-x} \mathrm{Mn}_{x}$ films grown at different temperatures are investigated: crystalline columns with and without partial relaxation through the formation of a pair of dislocations, amorphous nanocolumns and $\mathrm{Ge}_{3} \mathrm{Mn}_{5}$ clusters. For this purpose, highly sensitive SQUID magnetometry as well as EPR was thoroughly used.

\section{Crystalline $(\mathrm{Ge}, \mathrm{Mn})$ nanocolumns / Ge(001)}

Magnetic measurements on diluted $\mathrm{Ge}_{0.999} \mathrm{Mn}_{0.001}$ films grown at $100^{\circ} \mathrm{C}$ are first reported. Only a paramagnetic signal at low temperature by SQUID and the six isotropic hyperfine absorption lines characteristic of paramagnetic Mn atoms in EPR spectra (not shown) could be measured. This result is in good agreement with TEM observations and EXAFS measurements. Indeed the absence of any contrast in TEM images rules out the presence of nanocolumns and EXAFS oscillations could be fitted with a single substitutional environment.
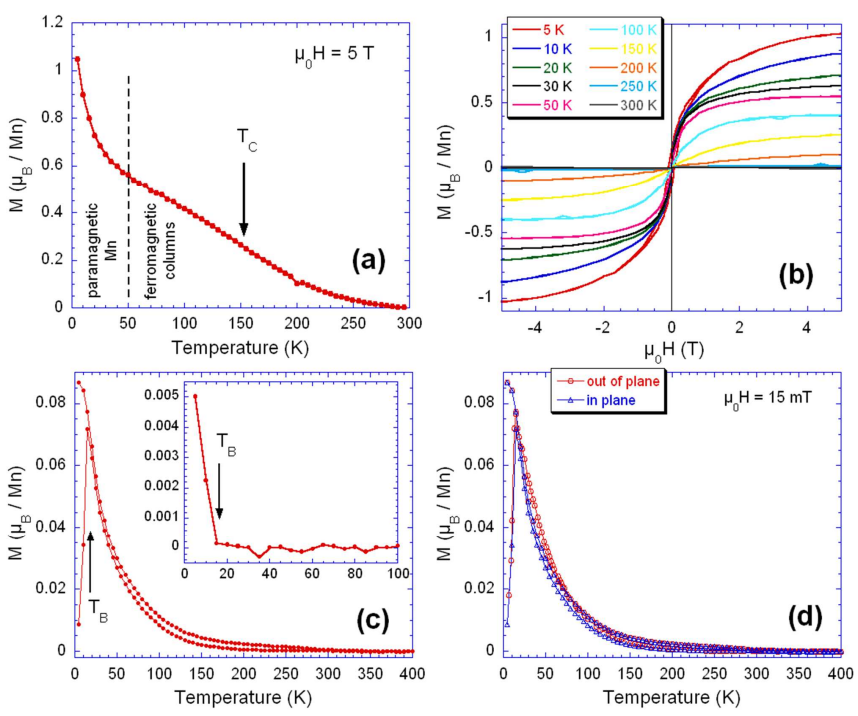

FIG. 58 (Color online) Magnetic characterization of a $\mathrm{Ge}_{0.9} \mathrm{Mn}_{0.1}$ film grown at $100^{\circ} \mathrm{C}$. (a) Temperature dependence of the saturation magnetization (in $\mu_{B} / \mathrm{Mn}$ ). The applied field is $5 \mathrm{~T}$. (b) Magnetization curves for different temperatures and (c) ZFC-FC measurements, pointing to $T_{\mathrm{C}} \approx 150 \mathrm{~K}$ of nanocolumns. The in-plane applied field is $0.015 \mathrm{~T}$. Inset: Magnetic remanence after maximum field cooling under $5 \mathrm{~T}$. (d) ZFC measurements performed with the field parallel and perpendicular to the film plane. From Jain et al. 2010.

For more concentrated films grown at $T_{\mathrm{g}}=100^{\circ} \mathrm{C}$, small nanocolumns spanning the whole film thickness are observed by TEM. Moreover they are well crystalline and in perfect epitaxial relationship with the Ge matrix (see Fig. 57). Assuming that the Mn concentration in the Ge matrix is below $0.05 \%$, the resulting $\mathrm{Mn}$ content in 
the columns is close to $50 \%$. SQUID measurements performed on a $\mathrm{Ge}_{0.9} \mathrm{Mn}_{0.1}$ film are reported in Fig. 58, Two different contributions in Fig. 58 (a) can be identified: a paramagnetic signal from isolated Mn atoms and the FM nanocolumns. It has been shown recently that the paramagnetic Mn atoms are located within a thin shell around nanocolumns (Dalmas de Réotier et al. 2015). The $T_{\mathrm{C}}$ magnitude of the columns is of the order of $150 \mathrm{~K}$, according to Figs. 58(b,c) (Jain et al., 2010). In Fig.58(b), magnetization curves are reversible above $15 \mathrm{~K}$, also confirmed by ZFC-FC curves and the dependence of remanence with temperature [Fig.58(c)]. These nanocolumns are superparamagnetic with a blocking temperature of $15 \pm 5 \mathrm{~K}$. Moreover the narrow shape of the ZFC peak is related to the narrow size distribution of nanocolumns grown in this temperature range (see Fig.51]. To determine the magnetic anisotropy of these columns, ZFC-FC measurements were performed with the field parallel and perpendicular to the film plane. The susceptibility perpendicular to the plane seems slightly higher than that in the plane but a strong diamagnetic signal from the substrate and paramagnetic signal from diluted Mn atoms makes it difficult to quantify precisely this anisotropy.
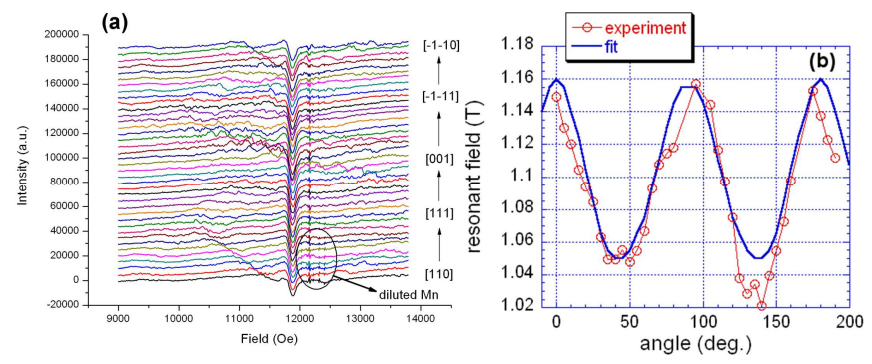

FIG. 59 (Color online) (a) EPR absorption spectra of a $\mathrm{Ge}_{0.9} \mathrm{Mn}_{0.1}$ film grown at $100^{\circ} \mathrm{C}$ as a function of the static field orientation at $5 \mathrm{~K}$. The angle is defined between the [110] direction and the applied magnetic field. The working frequency is $34 \mathrm{GHz}$ ( $Q$-band setup). Two different contributions can be observed: a FM broad peak of weak anisotropy and six hyperfine peaks from diluted paramagnetic Mn atoms. (b) Angular dependence of the resonance field. From Jain et al. 2010

EPR technique is hence used to differentiate the FM signal of nanocolumns from the paramagnetic diluted Mn atoms and estimate magnetic anisotropy Jain et al. 2010). The EPR spectra are observed as a function of the angle of applied magnetic field. The field is applied out-of-plane from the direction [110] to direction [1̄10] passing through the [001] direction. In Fig.59 the EPR spectra of a $\mathrm{Ge}_{0.9} \mathrm{Mn}_{0.1}$ film with crystalline nanocolumns exhibit the hyperfine Mn lines corresponding to isolated paramagnetic $\mathrm{Mn}$ atoms and also a weak FM line attributed to nanocolumns. The angular dependence of the resonance field in Fig. 59(b) is fitted using the Smit-Beljers formalism (Smit and Beljers, 1955). For this purpose, it is assumed that nanocolumns are noninteracting and single domain at $5 \mathrm{~K}$. The magnetic anisotropy energy of a single nanocolumn is written as: $F=K_{2} m_{z}^{2}+K_{4}\left(m_{x}^{2} m_{y}^{2}+m_{x}^{2} m_{z}^{2}+m_{y}^{2} m_{z}^{2}\right)$ where $x, y$, and $z$ axes are the [100], [010], and [001] crystal axes respectively. $m_{x}, m_{y}$, and $m_{z}$ are the coordinates of a unit vector along the column magnetization direction and $K_{2}$ and $K_{4}$ are the second and fourth order magnetic anisotropy constants. Anisotropy fields are thus defined as $\mu_{0} H_{a 2}=2 K_{2} / M_{S}$ and $\mu_{0} H_{a 4}=2 K_{4} / M_{S}$. From EPR data, the anisotropy fields can be deduced from EPR data: $\mu_{0} H_{a 2} \simeq-0.09 \mathrm{~T}$ for the second order and $\mu_{0} H_{a 4} \simeq-0.11 \mathrm{~T}$ for the fourth order with $\gamma / \gamma_{e} \simeq 1.07$ (where $\gamma_{e}$ is the gyromagnetic ratio of a free electron). Hence, the nanocolumns exhibit a perpendicular uniaxial anisotropy and a cubic anisotropy with the easy axis along [111]. The presence of cubic anisotropy supports the crystallinity of nanocolumns. Also, cubic anisotropy and uniaxial anisotropy constants are of the same order of magnitude making the two crystal axes [110] and [001] close in energy. It thus explains the small difference between the ZFC curves for the field parallel and perpendicular to the film plane in the SQUID measurements of Fig.58(d). Considering the saturation magnetization of these small nanocolumns $M_{S}=140 \pm 20$ $\mathrm{kA} / \mathrm{m}$, the uniaxial anisotropy constant can be calculated as $K_{2} \simeq-0.63 \times 10^{4} \mathrm{~J} / \mathrm{m}^{3}$ which corresponds to shape anisotropy $\mu_{0} M_{S}^{2} / 4 \simeq 0.62 \times 10^{4} \mathrm{~J} / \mathrm{m}^{3}$ assuming that nanocolumns are infinite cylinders (Aharoni, 1996).

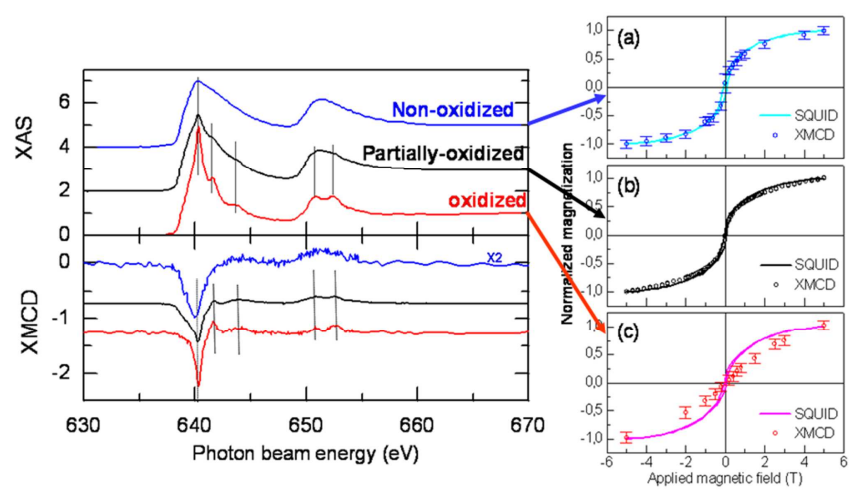

FIG. 60 (Color online) Isotropic XAS spectra at the $\mathrm{Mn} L_{2,3}$ edge and corresponding XMCD signal recorded at $5 \mathrm{~K}$ under $5 \mathrm{~T}$. Data were acquired in the total electron yield mode. Measurements were performed on a $\mathrm{Ge}_{0.9} \mathrm{Mn}_{0.1}$ film grown at $100^{\circ} \mathrm{C}$ exhibiting different surface states: nonoxidized, partially oxidized, and oxidized. The spectra are shifted vertically for clarity. The XMCD signal recorded at the $\mathrm{Mn} L_{3}$ edge is then compared with the SQUID signal at $5 \mathrm{~K}$. Both XMCD and SQUID signals are normalized to their maximum value. Adapted from Tardif et al., 2010a.

Finally in order to verify that the magnetic signal detected by SQUID magnetometry really comes from Mnrich nanocolumns, XMCD measurements were carried out at the $\mathrm{Mn} L_{2,3}$ edge (Ahlers et al. 2009: Gambardella et al. 2005). In Fig. 60, when the $\mathrm{Ge}_{0.9} \mathrm{Mn}_{0.1}$ film is properly capped with a $\mathrm{Si} / \mathrm{Ge}$ layer to prevent surface 
oxidation (Tardif et al., 2010a), the XMCD signal fits the SQUID measurements. Moreover the magnetic moment per Mn atom deduced by sum rules (Thole et al. $1992)$ from XMCD data also corresponds well to the magnetic moment measured by SQUID: $\simeq 1 \mu_{B} / \mathrm{Mn}$. Electrical properties have been intensively investigated in such (Ge,Mn) films grown on semi-insulating GaAs substrates (Yu et al. 2010). A negative giant MR signal has been detected at low temperature due to spin scattering on FM nanocolumns. In addition, two positive MR contributions were observed: the first one arises from ordinary or Lorentz MR and the second one was attributed to geometrically enhanced MR as a consequence of the conductivity contrast between the nanocolumns and the matrix (Solin et al. . 2000). Then AHE was demonstrated at low temperature but the interpretation of magnetotransport in such an inhomogeneous system remains a challenging task (Yu et al., 2011).

\section{Crystalline $(\mathrm{Ge}, \mathrm{Mn})$ nanocolumns with partial relaxation / $\mathrm{Ge}(001)$}

$\mathrm{Ge}_{0.94} \mathrm{Mn}_{0.06}$ films grown in a narrow temperature range around $130^{\circ} \mathrm{C}$ exhibit partial lattice relaxation (see Fig. 57) and ferromagnetism above RT up to $400 \mathrm{~K}$ as shown in Fig. 61 (Jamet et al., 2006). This high- $T_{\mathrm{C}}$ FM behavior was also observed in nanowires and quantum dots (Cho et al., 2008b, Kazakova et al., 2005, van der Meulen et al. 2009, Xiu et al., 2010a).

Figure 61(a) shows the temperature dependence of the magnetization at $2 \mathrm{~T}$, measured by SQUID magnetometry. The magnetic moment per Mn atom is $4.7 \mu_{B}$ at $3 \mathrm{~K}$. This value is close to $5 \mu_{B}$ expected for isolated $\mathrm{Mn}^{2+}$ ions according to Hund's rule. The large magnetization at high temperature is consistent with a FM phase with $T_{\mathrm{C}}>400 \mathrm{~K}$. ZFC-FC curves superimpose from 3 to $300 \mathrm{~K}$ [Fig. 61(b)], thus ruling out the presence of superparamagnetic particles except if their blocking temperature exceeds $300 \mathrm{~K}$. Considering the Mn distribution in the GeMn films, this high $T_{\mathrm{C}} \mathrm{FM}$ phase can be attributed to the nanocolumns. In the low-temperature range, the magnetization increases when decreasing the temperature [inset in Fig.61(a)]. The corresponding saturation magnetization is small $(\simeq 9 \mathrm{kA} / \mathrm{m})$, and the additional susceptibility is described by a Curie-Weiss temperature between 10 and $15 \mathrm{~K}$. Similar behavior was reported for a strongly diluted $\mathrm{Ge}_{1-x} \mathrm{Mn}_{x}$ layer. Assuming that isolated Mn atoms are present in the film, all magnetically active with a magnetic moment of $3 \mu_{B}$ (Schulthess and Butler, 2001), the Mn concentration in the nanocolumns reaches $33.7 \%$ (their composition is close to $\mathrm{Ge}_{2} \mathrm{Mn}$ ). The magnetic moment per $\mathrm{Mn}$ atom is close to $4.9 \mu_{B}$ from the saturation magnetization $\simeq 680 \mathrm{kA} / \mathrm{m}$. Magnetization loops [Fig.61(c)] all exhibit a pronounced S shape, a low remanent magnetization and a low coercive field [Fig.61(d)]: in-plane and out-of-plane directions act as hard magnetic axes. The saturation is easier with the
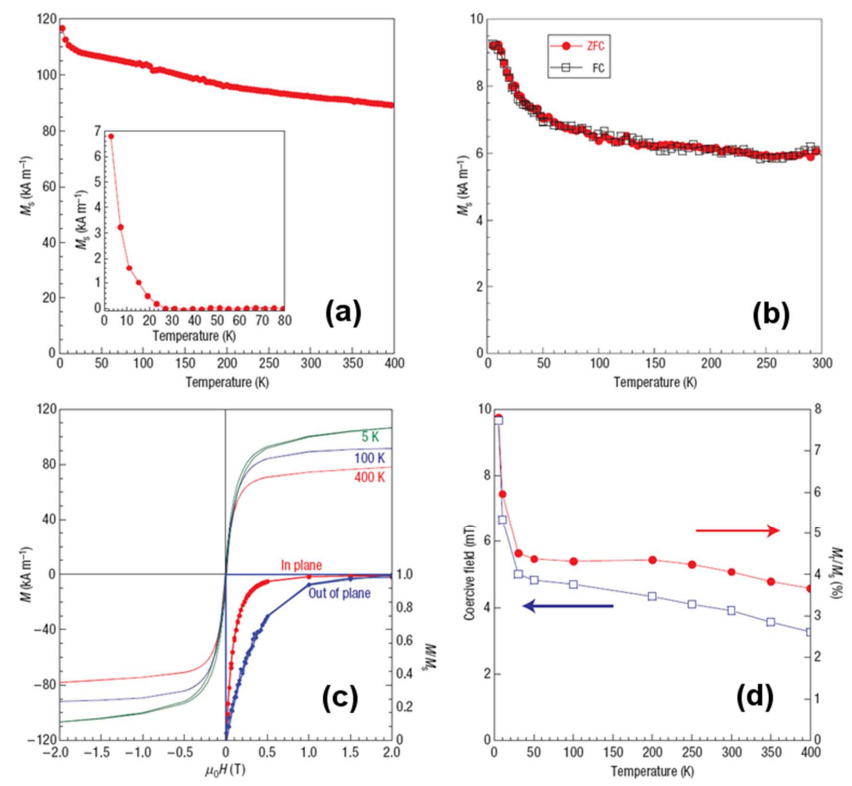

FIG. 61 (Color online) Magnetic characterization of $\mathrm{Ge}_{0.94} \mathrm{Mn}_{0.06}$ films grown around $130^{\circ} \mathrm{C}$. (a) Temperature dependence of the saturation magnetization measured at $2 \mathrm{~T}$. The inset shows the extrapolated matrix signal at low temperature after subtracting the nanocolumns magnetic signal. (b) ZFC-FC measurements performed at $0.01 \mathrm{~T}$. Both curves are superimposed. (c) Magnetization loops at 5, 100, and $400 \mathrm{~K}$, after subtracting the diamagnetic contribution from the substrate. The inset demonstrates the easier saturation in-plane at $250 \mathrm{~K}$. (d) Coercive field $\left(\mu_{0} H_{C}\right)$ and remanent magnetization $\left(M_{r} / M_{S}\right)$ versus temperature. $\mu_{0} H_{C}$ and $M_{r} / M_{S}$ are given with a precision of the order of $10 \%$. From Jamet et al. 2006

field applied in-plane [inset in Fig.61(c)].

Nanocolumns are very close to each other and a strong magnetostatic or carrier mediated coupling is expected. Energy calculations using the Fourier formalism (Beleggia et al. 2004) were performed for an infinite square lattice of nanocolumns defined by the average experimental parameters (spacing $10 \mathrm{~nm}$, diameter $3 \mathrm{~nm}$ and height $80 \mathrm{~nm}$ ), taking into account the long-range dipolar interaction and dipolar self-energy of the nanocolumns. Three configurations have been tested: saturated out-of-plane $(\uparrow \uparrow)$, saturated in-plane $(\rightarrow \rightarrow)$ and antiparallel out-ofplane with $M=0(\uparrow \downarrow)$. Results are given in units of $\mu_{0} M_{S}^{2} V / 2$ where $V$ is the volume of the nanocolumns and $M_{S}$ their magnetization at saturation. The smallest energy, $E_{\uparrow \downarrow} \simeq 0.01$, corresponds to the antiparallel out-of-plane configuration: dipolar interactions favor an antiparallel arrangement of first neighbor nanocolumns. This finding is in good agreement with the low remanent magnetizations, low coercive fields and S shaped inplane and out-of-plane magnetization curves measured by SQUID. In real samples, the remanent magnetization is not zero because nanocolumns are randomly distributed, leading to many frustrated magnetic configu- 
rations. The model however fails to predict the right saturated configuration: we calculate $E_{\rightarrow \rightarrow}=0.46$ and $E_{\uparrow \uparrow}=0.08$, while experimentally the magnetization is easier to saturate in-plane $\left(E_{\rightarrow \rightarrow}<E_{\uparrow \uparrow}\right)$. Thus an additional in-plane magneto-crystalline anisotropy has to be considered, competing with shape anisotropy, which favors the in-plane saturated state. This behavior has previously been observed in arrays of electrodeposited cobalt nanowires (Darques et al. 2004). In (Ge,Mn) nanocolumns, this magnetocrystalline anisotropy originates from the broken in-plane cubic symmetry (columns are fully strained in only one direction) leading to a natural uniaxial anisotropy combined with large magnetoelastic effects (columns are $4 \%$ contracted). This feature also explains the very high (at least higher than $400 \mathrm{~K}$ ) blocking temperature of nanocolumns.

As previously discussed in III-V and II-VI FM semiconductors (Dietl et al., 2001, Kossacki et al., 2004), strain can modify the valence band dispersion and induce a large magnetic anisotropy. Carrier mediated FM coupling between the nanocolumns would also favor an in-plane saturated state. Note that in calculating the self-energy that the column aspect ratio can be overestimated since the columns are not perfect cylinders. Finally, these (Ge,Mn) films grown on Ge exhibit large positive MR attributed to geometrically enhanced MR (Solin et al. 2000) and more importantly AHE that might be attributed to the spin polarization of holes (Jamet et al. 2006). That makes this system a promising candidate for spintronics applications.

\section{Amorphous (Ge,Mn) nanocolumns / Ge(001)}

For $\mathrm{Ge}_{1-x} \mathrm{Mn}_{x}$ films grown at higher temperatures $\left(T>150^{\circ} \mathrm{C}\right)$ and high Mn concentration $(\geq 10 \%)$, amorphous nanocolumns are observed in TEM (see Fig.57). As for small crystalline nanocolumns grown at $100^{\circ} \mathrm{C}$ (see Sec.VI.D.1), the Mn concentration in the columns is close to $50 \%$. These amorphous nanolumns have $T_{\mathrm{C}}$ close to $170 \mathrm{~K}$. The contribution from isolated paramagnetic Mn atoms is still observed at low temperature, as shown in Fig.62.

In ZFC-FC curves [Fig. 62(b)] two peaks are present at around $25 \mathrm{~K}$ and $75 \mathrm{~K}$ in the $\mathrm{ZFC}$ curve as well as a weak contribution from $\mathrm{Ge}_{3} \mathrm{Mn}_{5}$ clusters. Those peaks are in agreement with the broad size distribution observed by TEM (see Fig.51).

The magnetic anisotropy of these columns is studied by EPR (Jain et al. 2010). The data are shown in Fig.63 and the angular dependence of the resonance field is fitted using the Smit-Beljers formalism (Smit and Beljers, 1955) assuming that the nanocolumns are noninteracting and single domain. A single perpendicular uniaxial anisotropy is found; the corresponding anisotropy field is $\mu_{0} H_{a 2} \simeq-0.09 \mathrm{~T}$ and $\gamma / \gamma_{e} \simeq 1.24$. From the saturation magnetization of amorphous nanocolumns $M_{S}=220 \pm$ $20 \mathrm{kA} / \mathrm{m}$, the anisotropy energy density is calculated to
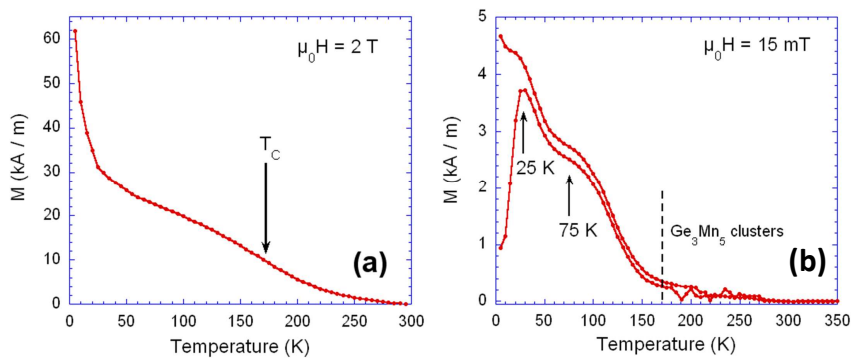

FIG. 62 (Color online) Magnetic characterization of a $\mathrm{Ge}_{0.9} \mathrm{Mn}_{0.1}$ film grown above $150^{\circ} \mathrm{C}$ with amorphous nanocolumns. (a) Temperature dependence of the saturation magnetization in the magnetic field of $2 \mathrm{~T}$ applied in the film plane along the [110] direction. (b) ZFC-FC measurements performed at $0.015 \mathrm{~T}$. The $\mathrm{ZFC}$ curve exhibits two peaks with blocking temperatures around 25 and $75 \mathrm{~K}$, and points to $T_{\mathrm{C}} \simeq 170 \mathrm{~K}$ of nanocolumns. Nonzero magnetization at higher temperatures is due to $\mathrm{Ge}_{3} \mathrm{Mn}_{5}$ NCs. From Jain et al. 2010.
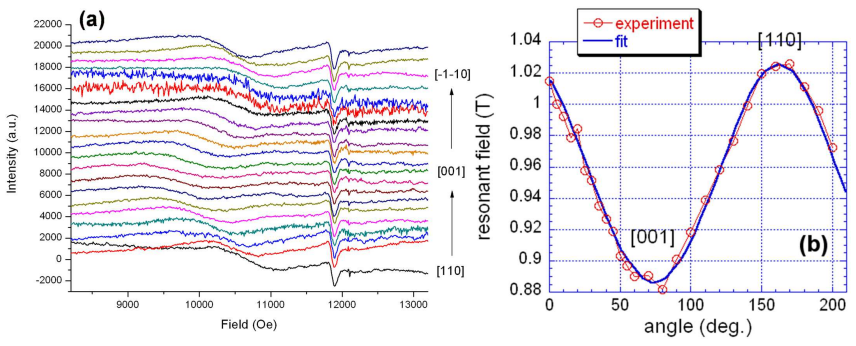

FIG. 63 (a) EPR absorption spectra of a $\mathrm{Ge}_{0.9} \mathrm{Mn}_{0.1}$ film grown at $150^{\circ} \mathrm{C}$ as a function of the static field orientation at $5 \mathrm{~K}$. The angle is defined between the [110] direction and the applied magnetic field. The working frequency is $34 \mathrm{GHz}$ (Q-band set-up). A single FM broad peak of weak anisotropy is observed. (b) Angular dependence of the resonance field. From Jain et al. 2010.

be $K_{2} \simeq-1.1 \times 10^{4} \mathrm{~J} / \mathrm{m}^{3}$. This value corresponds to that of shape anisotropy $\mu_{0} M_{S}^{2} / 4=1.5 \times 10^{4} \mathrm{~J} / \mathrm{m}^{3}$ of nanocolumns assuming their aspect ratio is high Aharoni 1996). As expected for amorphous nanocolumns, no cubic magneto-crystalline contribution is found. In the case of uniaxial magnetic anisotropy, the blocking temperature of nanocolumns can be easily estimated using the classical Néel-Brown formula (Jamet et al., 2001): $K V=25 k_{B} T_{B}$, where $K$ is the anisotropy constant, $V$ the columns volume, $k_{B}$ the Boltzmann constant and $T_{B}$ the blocking temperature. From the average diameter and length of nanocolumns ( $6 \mathrm{~nm}$ and $80 \mathrm{~nm}$ respectively) given by TEM observations, one can estimate their volume and the blocking temperature: $T_{B} \simeq 72 \mathrm{~K}$ which corresponds to the second broad peak in the ZFC curve. The first peak with a maximum close to $25 \mathrm{~K}$ can correspond either to shorter magnetic nanocolumns with an average length of $\simeq(25 / 72) \times 80 \mathrm{~nm}=28 \mathrm{~nm}$ or to the manifestation of a disordered magnetic state within the 
thin shell around nanocolumns and containing substitutional Mn atoms (Dalmas de Réotier et al., 2015). In the first assumption it means that increasing the growth temperature partly activates Mn diffusion along the growth direction, which leads to the formation of elongated clusters rather than continuous nanocolumns spanning the whole film thickness.

\section{4. $\mathrm{Ge}_{3} \mathrm{Mn}_{5}$ clusters / $\mathrm{Ge}(001)$}

The growth of $\mathrm{Ge}_{1-x} \mathrm{Mn}_{x}$ films at high temperature $\left(>180^{\circ} \mathrm{C}\right)$ on $\mathrm{Ge}(001)$ or annealing $\mathrm{Ge}_{1-x} \mathrm{Mn}_{x}$ films grown at low temperature above $600^{\circ} \mathrm{C}$ leads to the formation of randomly distributed spherical $\mathrm{Ge}_{3} \mathrm{Mn}_{5}$ clusters. The RHEED pattern of annealed samples is very similar to that of Ge as in HRTEM the clusters are observed away from the surface (Fig.57).

The structure of $\mathrm{Ge}_{3} \mathrm{Mn}_{5}$ clusters was investigated using grazing incidence SXRD. The mean cluster diameter was estimated to be $10.6 \pm 1 \mathrm{~nm}$ in agreement with TEM observations. Moreover $97 \%$ of the clusters have their $c$-axis perpendicular to the film plane and $3 \%$ in plane. Finally, by searching for all possible $\mathrm{Ge}_{3} \mathrm{Mn}_{5}$ reflections in the plane parallel to the sample surface, a slight in-plane distortion of the hexagonal lattice was found (Jain et al. 2011). Magnetic properties were studied using SQUID magnetometry and EPR. Temperaturedependent magnetization measurements show the presence of two magnetic phases: the $\mathrm{Ge}_{3} \mathrm{Mn}_{5}$ with $T_{\mathrm{C}}$ of $300 \pm 5 \mathrm{~K}$ and the paramagnetic contribution of isolated $\mathrm{Mn}$ atoms. Hysteresis curves at $5 \mathrm{~K}$ show that most of $\mathrm{Ge}_{3} \mathrm{Mn}_{5}$ clusters exhibit perpendicular magnetic anisotropy. Since the clusters are spherical, perpendicular anisotropy arises from magnetocrystalline anisotropy. This result is in good agreement with XRD data. Indeed, bulk $\mathrm{Ge}_{3} \mathrm{Mn}_{5}$ crystal is hexagonal with uniaxial magnetic anisotropy along the $c$-axis. From hysteresis loops recorded with the field perpendicular to the film plane, the fraction of magnetic signal from $\mathrm{Ge}_{3} \mathrm{Mn}_{5}$ clusters is estimated to be $70 \%$. The remaining signal likely comes from amorphous Mn-rich precipitates with very low coercive field. From the anisotropy field $\mu_{0} H_{a} \simeq 0.6 \mathrm{~T}$ and using the bulk $M_{S}$ value $(1100 \mathrm{kA} / \mathrm{m})$ (Forsyth and Brown, 1990), the anisotropy constant can be calculated: $K=\mu_{0} H_{a} M_{S} / 2 \simeq 3.3 \times 10^{5} \mathrm{~J} / \mathrm{m}^{3}$ which is less than the reported bulk value $4.2 \times 10^{5} \mathrm{~J} / \mathrm{m}^{3}$ (Tawara and Sato, 1963). This feature is explained by the in-plane distortion of the $\mathrm{Ge}_{3} \mathrm{Mn}_{5}$ crystal observed by XRD which is due to the epitaxy on the germanium matrix. It introduces an additional in-plane magnetoelastic component to magnetic anisotropy. Finally the same anisotropy field and perpendicular anisotropy were obtained from EPR measurements in good agreement with SQUID data (Jain et al. 2011).

\section{SPINODAL NANODECOMPOSITION IN (Ge,Fe)}

\section{A. Introduction}

Apart from Mn other TM atoms such as Cr, Co or Fe have also been incorporated into Ge films to obtain FM properties (Kim et al., 2005, Kioseoglou et al., 2004, Tsui et al. , 2004). In particular, FM order was predicted in Fe-doped germanium films (Weng and Dong, 2005, Zhou et al., 2004) and experimentally demonstrated in Fe-implanted films (Venugopal et al., 2002), Fe-doped germanium nanowires (Cho et al., 2008a) or quantum dots (Xiu et al. $2010 \mathrm{~b}$ ) as well as in Fe-doped bulk Ge single crystals (Choi et al. 2003 ).

In order to enhance the solubility limit of Fe, LTMBE was used to grow $\mathrm{Ge}_{1-x} \mathrm{Fe}_{x}$ films with $x$ up to $17.5 \%$ (Shuto et al. 2006b). In this section, we describe the growth, characterization, and magnetic properties of such $\mathrm{Ge}_{1-x} \mathrm{Fe}_{x}$ thin films, in particular, the relation between growth or annealing temperatures, defect formation, Fe distribution, and resulting magnetism (Shuto et al. 2006a b, 2007, Wakabayashi et al., 2014a b).

\section{B. MBE growth}

$\mathrm{Ge}_{1-x} \mathrm{Fe}_{x}$ thin films were grown on Ge (001) substrates by LT-MBE. After a LT Ge buffer layer was grown at the substrate temperature $T_{\mathrm{g}}$ of $100^{\circ} \mathrm{C}$, a 16 -nm-thick $\mathrm{Ge}_{1-x} \mathrm{Fe}_{x}$ film was grown at $T_{\mathrm{g}}=100,200,300$, or $400^{\circ} \mathrm{C}$. The Fe content $(x)$ was varied from 2.0 to $24.0 \%$. In situ RHEED was used to monitor the crystallinity and surface morphology of the Ge buffer layer and the $\mathrm{Ge}_{1-x} \mathrm{Fe}_{x}$ film during the epitaxy. Although the diffraction pattern of the LT Ge buffer layer revealed intense and sharp $2 \times 2$ streaks, the pattern was quickly changed at the initial stage of $\mathrm{Ge}_{1-x} \mathrm{Fe}_{x}$ epitaxy. The $\mathrm{Ge}_{1-x} \mathrm{Fe}_{x}$ films grown at $T_{\mathrm{g}}=100$ and $200^{\circ} \mathrm{C}$ showed $2 \mathrm{D}$ growth mode and exhibited diamond-type crystal structure.

When the $\mathrm{Ge}_{1-x} \mathrm{Fe}_{x}$ film was grown at $T_{\mathrm{g}}=300$ (or $400^{\circ} \mathrm{C}$ ), a spotty pattern was clearly observed in the RHEED image, indicating a 3D growth mode or surface roughening. Thus, the growth mode of the $\mathrm{Ge}_{1-x} \mathrm{Fe}_{x}$ film was changed from the $2 \mathrm{D}$ mode to the $3 \mathrm{D}$ mode between $T_{\mathrm{g}}=200$ and $300^{\circ} \mathrm{C}$. This growth mode change at around $T_{\mathrm{g}}=300^{\circ} \mathrm{C}$ is caused by the precipitates of FeGe FM compounds in the film (crystallographic phase separation), as discussed next.

\section{Structural and chemical characterization}

Figure 64(a) shows a HRTEM image of a $\mathrm{Ge}_{1-x} \mathrm{Fe}_{x}$ film $(x=9.5 \%)$ grown at $T_{\mathrm{g}}=200^{\circ} \mathrm{C}$, projected along the exact direction of Ge[110]. The image indicates that the $\mathrm{Ge}_{1-x} \mathrm{Fe}_{x}$ layer was homogeneously and epitaxially grown on the Ge buffer layer without any threading dislocations, and that the surface was atomically flat with a height of roughness less than $1 \mathrm{~nm}$. These features were 
consistent with the RHEED observations, as mentioned earlier. However, when the lattice image was projected along the angle slightly tilted from the Ge[110] direction, clusterlike dark contrast regions appeared in the epitaxially grown $\mathrm{Ge}_{1-x} \mathrm{Fe}_{x}$ layer, as shown in Fig.64(b). It is well recognized in TEM observations that the contrast due to the difference in chemical compositions is enhanced when the projection direction is slightly tilted from the exact zone axis. Using spatially resolved transmission electron diffraction (TED) and EDS, the crystal structure and chemical composition of the dark region [denoted as *1 in Fig. 64(b)] and surrounding bright region [denoted as $*_{2}$ in Fig. 64(b)] were investigated. The TED image of point $* 1$ (the right inset) showed the diffraction pattern of the diamond structure projected from its [110] direction with weak extra spots caused by stacking fault defects. On the contrary, point $*_{2}$ (the left inset) in the bright region exhibited the diffraction pattern of the diamond structure without any extra spots. This means that no other crystal structures except the diamond crystal structure were formed both in the dark and in the bright regions, although the dark region included small stacking fault defects.

The local Fe composition at point $* 1$ measured by EDS was $\sim 12 \%$, which is higher than that $(\sim 4 \%)$ at point ${ }^{*} 2$. Therefore, the contrast in the HRTEM image shown in Fig. 64(b) was due to the nonuniform distribution of the Fe composition. Similar structural features were also observed for a $\mathrm{Ge}_{1-x} \mathrm{Fe}_{x}$ film with $x=6.0 \%$, in which dark and bright contrast regions appeared in the TEM image caused by nonuniform Fe distribution. Small stacking fault defects were also detected in the dark contrast regions. The local Fe composition in the dark and bright contrast regions of this film was about $8.0 \%$ and $1.5 \%$, respectively. Thus, both the Fe compositions in dark and bright contrast regions increased with increasing $x$.

Thus, RHEED, HRTEM, TED, and EDS studies lead to the conclusion that $\mathrm{Ge}_{1-x} \mathrm{Fe}_{x}$ films grown at $200^{\circ} \mathrm{C}$ on $\mathrm{Ge}(001)$ maintain the diamond crystal structure without any intermetallic Fe-Ge compounds or Fe inclusions. Similarly, the diamond structure is preserved under annealing (Ge,Fe) below $600^{\circ} \mathrm{C}$ (Wakabayashi et al. $\left.2014 \mathrm{a}\right)$. However, the films grown at $200^{\circ} \mathrm{C}$ or annealed exhibit a nonuniform distribution of Fe composition $x$, i.e., they undergo spinodal nanodecomposition in the form of the chemical phase separation.

It is worth noting that since EDS provides chemical information averaged over the specimen width, the amplitude of actual $x$ fluctuations can be even larger than implied by the values of $x$ determined for points $* 1$ and $* 2$.

\section{Magneto-optical characterization}

Figure 65(a) shows MCD spectra taken at $10 \mathrm{~K}$ in reflection configuration for $\mathrm{Ge}_{1-x} \mathrm{Fe}_{x}(x=9.5 \%)$ films grown at $T_{\mathrm{g}}=100,200,300$, and $400^{\circ} \mathrm{C}$, with magnetic

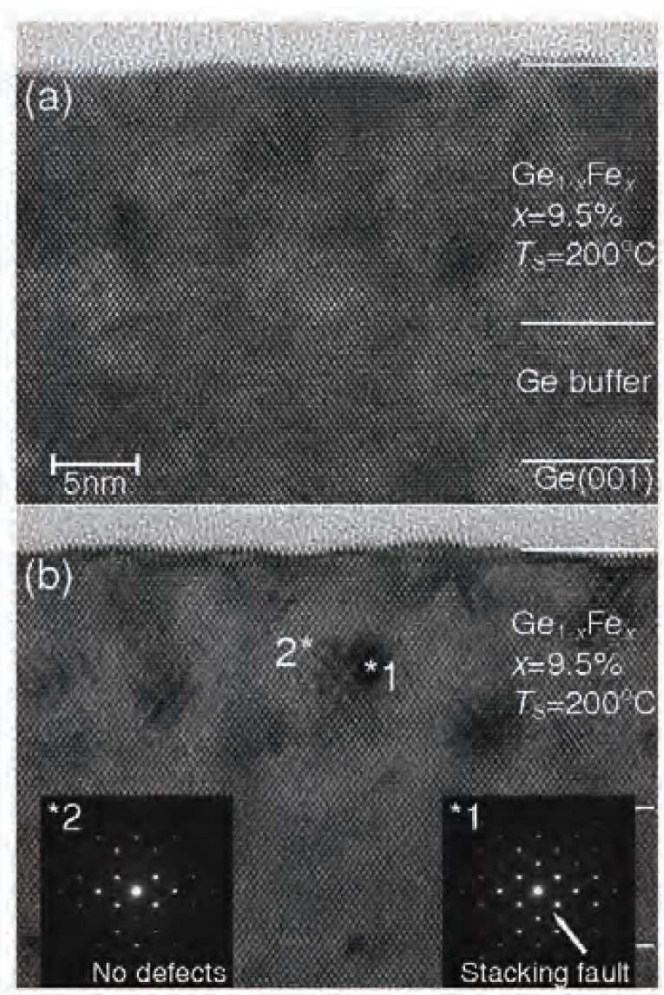

FIG. 64 HRTEM lattice images projected along (a) the exact direction of the Ge[110] axis and (b) the direction slightly tilted from the Ge[110] axis for $\mathrm{Ge}_{1-x} \mathrm{Fe}_{x}$ thin films with $x=$ $9.5 \%$ grown at $T_{\mathrm{g}}=200^{\circ} \mathrm{C}$. Insets: Diffraction images at the dark region $\left(*^{*} 1\right)$ and at the surrounding bright region $\left(*^{*}\right)$. From Shuto et al. 2007.

field perpendicular to the sample plane. As see, when the $\mathrm{Ge}_{1-x} \mathrm{Fe}_{x}$ film was grown at $T_{\mathrm{g}}=100$ ( or $200^{\circ} \mathrm{C}$ ), its MCD spectrum showed an $E_{1}$ peak whose intensity was significantly enhanced compared with that of bulk Ge [uppermost curve in Fig.65(a)], implying the presence of $s, p-d$ exchange splitting of bands. An offsetlike broad signal was also observed in the whole energy range examined here. The magnetic-field dependence of the MCD intensity at the critical point $E_{1}$ exhibited a FM hysteresis loop, and the hysteresis shape at any other photon energies in the offsetlike signal was identical with that at E1, indicating the $\mathrm{Ge}_{1-x} \mathrm{Fe}_{x}$ film grown at $T_{\mathrm{g}}=100-200^{\circ} \mathrm{C}$ was magnetically homogeneous without Fe-Ge FM precipitates. Note that the $E_{0}$ of the sample was not resolved due to light interference caused by adsorbed moisture on the sample surface (Shuto et al. 2006b).

When the $\mathrm{Ge}_{1-x} \mathrm{Fe}_{x}$ film was grown at $T_{\mathrm{g}}=300$ (or $400^{\circ} \mathrm{C}$ ), the $\mathrm{MCD}$ intensity at the critical point $\mathrm{E}_{1}$ was not enhanced at all, but the MCD spectrum of bulk Ge was overlapped with an offsetlike MCD signal: The magnitude of the $\mathrm{E}_{1}$ peak [denoted as $\delta$ shown in Fig.65(a)] that was measured from the bottom of the offsetlike signal level was equal to that of bulk Ge. The MCD hysteresis loop measured at $\mathrm{E}_{1}(2.30 \mathrm{eV})$ was not identical with that measured at other energy points (for exam- 

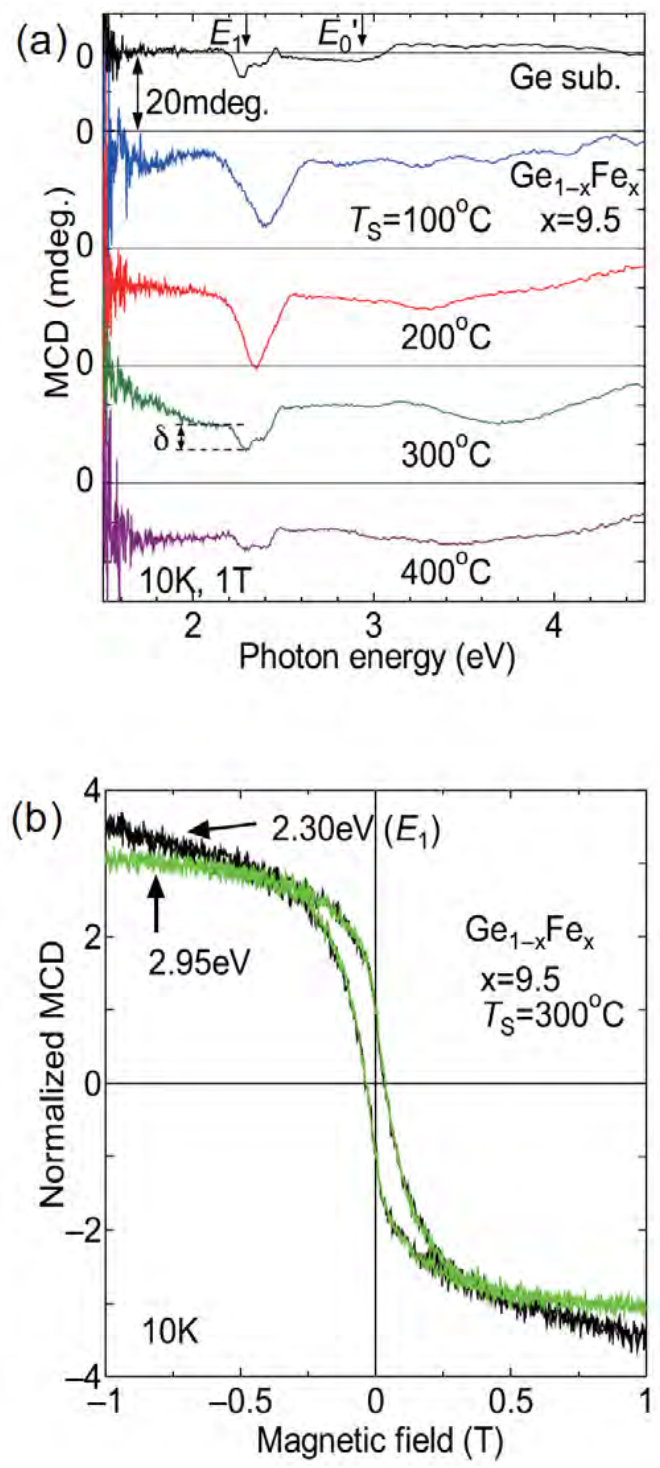

FIG. 65 (a) Growth temperature dependence of the MCD spectra of $\mathrm{Ge}_{1-x} \mathrm{Fe}_{x}(x=9.5 \%)$ films grown at $T_{\mathrm{g}}=100-$ $400^{\circ} \mathrm{C}$, measured at $10 \mathrm{~K}$ with magnetic field of $1 \mathrm{~T}$ perpendicular to the sample plane with reflection configuration. (b) Magnetic-field dependence of MCD intensities at $2.30 \mathrm{eV}$ $\left(E_{1}\right)$ and at other energy point $(2.95 \mathrm{eV})$ for the $\mathrm{Ge}_{1-x} \mathrm{Fe}_{x}$ $(x=9.5 \%)$ films grown at $T_{\mathrm{g}}=300^{\circ} \mathrm{C}$, where the data were normalized by their MCD intensities at the magnetic field of 0 T. Adapted from Shuto et al. 2006a.

ple, $2.95 \mathrm{eV}$ ) as shown in Fig. 65(b), indicating crystallographic phase separation. The MCD hysteresis loop at $\mathrm{E}_{1}$ can be divided into two components, i.e., the paramagnetic component of the host Ge matrix and the FM component of FM precipitates.

Figure 66 shows $T_{\mathrm{C}}$ values of $\mathrm{Ge}_{1-x} \mathrm{Fe}_{x}$ films as a function of Fe content $x$, where $T_{\mathrm{C}}$ was evaluated from the Arrott plots using temperature-dependent MCD hysteresis loops at the $E_{1}$ critical point.

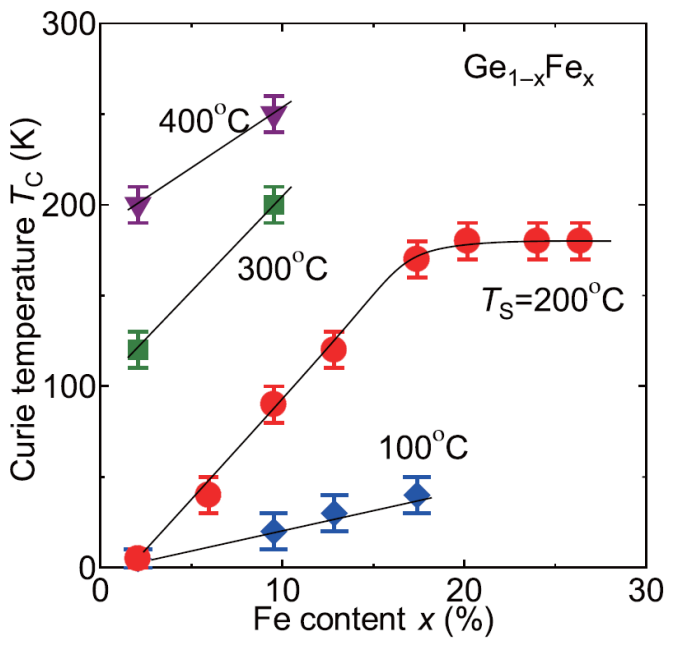

FIG. 66 Curie temperature $T_{\mathrm{C}}$ of $\mathrm{Ge}_{1-x} \mathrm{Fe}_{x}$ films as a function of $\mathrm{Fe}$ content $x$. $\mathrm{Ge}_{1-x} \mathrm{Fe}_{x}$ films were grown at $T_{\mathrm{g}}=100,200,300$, and $400^{\circ} \mathrm{C}$. From Shuto et al. 2006a

For the $\mathrm{Ge}_{1-x} \mathrm{Fe}_{x}$ films grown at $T_{\mathrm{g}}=200^{\circ} \mathrm{C}, T_{\mathrm{C}}$ linearly increased with increasing $x$ in the range between $x=0$ and $17.5 \%$. Above $x=17.5 \%, T_{\mathrm{C}}$ was saturated at around $170-180 \mathrm{~K}$ (Shuto et al. 2007 ). $T_{\mathrm{C}}$ of $\mathrm{Ge}_{1-x} \mathrm{Fe}_{x}$ films grown at $T_{\mathrm{g}}=100^{\circ} \mathrm{C}$ also linearly increased with increasing $x$, but $T_{\mathrm{C}}$ was significantly reduced in comparison with that of the samples grown at $T_{\mathrm{g}}=200^{\circ} \mathrm{C}$.

When $\mathrm{Ge}_{1-x} \mathrm{Fe}_{x}$ films were grown at $T_{\mathrm{g}}=300$ and $400^{\circ} \mathrm{C}$, their $T_{\mathrm{C}}$ values were higher than those of the samples grown at $T_{\mathrm{g}}=100$ and $200^{\circ} \mathrm{C}$. For example, $T_{\mathrm{C}}$ values were 120 and $200 \mathrm{~K}$ in the cases of $T_{\mathrm{g}}=300$ and $400^{\circ} \mathrm{C}$, respectively, even when $x$ was only $2.0 \%$. On the contrary, $T_{\mathrm{C}}$ values were below $10 \mathrm{~K}$ in the cases of $T_{\mathrm{g}}=100$ and $200^{\circ} \mathrm{C}$ with $x=2.0 \%$. This behavior of $T_{\mathrm{C}}$ at $T_{\mathrm{g}}=300$ and $400^{\circ} \mathrm{C}$ can be attributed to the precipitates of FM compounds described previously.

The apparent values of $T_{\mathrm{C}}^{(a p p)}$ not only increase with the growth temperature but can be enhanced by annealing (Wakabayashi et al. 2014a). In either case, the magnitude of $T_{\mathrm{C}}^{(a p p)}$ correlates with the nonuniformity of the Fe distribution and the stacking fault density (Wakabayashi et al. 2014a b). Meanwhile, channeling Rutherford backscattering and particle-induced x-ray emission measurements revealed that about $15 \%$ of the Fe atoms reside in the tetrahedral interstitial sites and that the substitutional $\mathrm{Fe}$ concentration, in agreement with the decomposition scenario, is barely correlated with the magnitude of $T_{\mathrm{C}}^{(a p p)}$ (Wakabayashi et al., 2014b). At the same time, all the $(\mathrm{Ge}, \mathrm{Fe})$ films show a weak spin-glasslike behavior in a low-temperature region (below $\sim 26 \mathrm{~K}$ ), which is insensitive to the annealing temperature. However, the ferromagnetism associated with the nonuniform Fe distribution dominates magnetic properties of the system.

In summary, studies of $\mathrm{Ge}_{1-x} \mathrm{Fe}_{x}$ as a function of epitaxy temperature and TM content clearly show an evo- 
lution of the alloy character from a dilute magnetic semiconductor to a decomposed system, first involving chemical and then crystallographic phase separation. This evolution is accompanied by changes in spectral dependences of MCD that is initially enhanced in the region of optical transitions specific to the host semiconductor but as nanodecomposition progresses this magnetization-dependent enhancement starts to extend over a wide spectral region indirectly pointing to a metallic character of Fe-rich regions. The apparent $T_{\mathrm{C}}$ values determined from the MCD hysteresis loops show a clear correlation with the degree of nanocomposition, i.e., with the upper limit of local TM concentrations as well as with the appearance of structural defects.

\section{SPINODAL NANODECOMPOSITION IN $(\mathrm{Zn}, \mathrm{Cr}) \mathrm{Te}$}

\section{A. Introduction}

Among various DMSs, Cr-doped II-VI compounds have been studied for a long time, attracting attention due to their peculiar magnetic and magnetooptical properties. Excitonic magnetospectroscopy indicated that the $p-d$ exchange interaction is FM (Mac et al., 1996), in contrast to the AF interaction in Mn-doped II-VI compounds. According to the Schrieffer-Wolff transformation, the FM $p-d$ interaction results from the position of the $\mathrm{Cr} 3 d$ donor level above the top of the valence band (Kacman, 2001; Mizokawa and Fujimori, 1997).

Concerning the spin-dependent coupling between $\mathrm{Cr}$ spins in (II,Cr) VI, it was theoretically predicted that the superexchange interaction becomes FM for $d^{4}$ ions in the tetrahedral crystal field (Blinowski et al. 1996). Later, a FM ordering of $\mathrm{Cr}$ spins in II-VI compounds was found by DFT first-principle calculations (Sato and KatayamaYoshida, 2002). Hence, the theoretically expected ferromagnetism can be understood in the picture of doubleexchange interaction between the $\mathrm{Cr} 3 d$ electrons in the localized level within the band gap (Fukushima et al. 2004 Sato and Katayama-Yoshida, 2002) or as FM superexchange interaction of highly localized $\mathrm{Cr}$ spins (Blinowski et al. 1996). In either case, the FM interaction between Cr spins is short-range, extending over few nearest-neighbors.

Experimentally, the FM behavior was observed in $(\mathrm{Zn}, \mathrm{Cr}) \mathrm{Te}$ (Saito et al., 2002$)$ and (Zn,Cr)Se (Karczewski et al. 2003) with $\mathrm{Cr}$ contents reaching a few percent, and later ferromagnetism at RT was achieved in $\mathrm{Zn}_{1-x} \mathrm{Cr}_{x} \mathrm{Te}$ with a high $\mathrm{Cr}$ content $x=0.2$ (Saito et al., 2003). The FM transition temperature $T_{\mathrm{C}}$ was reported to increase almost linearly with $\mathrm{Cr}$ content $x$, reaching $300 \mathrm{~K}$ at $x=0.2$, in agreement with the $a b$ initio prediction (Fukushima et al., 2004). In addition, the intrinsic nature of ferromagnetism appeared to be confirmed by the similar magnetic-field dependences of magnetization and MCD (Saito et al., 2003). However, according to Karczewski et al., 2003 , the ferromagnetism in $\mathrm{Zn}_{1-x} \mathrm{Cr}_{x} \mathrm{Se}$ resulted from the precipitation of a FM compound, as $T_{\mathrm{C}}$ was independent of $x$.

It was also found that the FM properties of $(\mathrm{Zn}, \mathrm{Cr}) \mathrm{Te}$ were significantly affected by the codoping of donor or acceptor impurities; the ferromagnetism was suppressed by codoping with acceptor impurity nitrogen $(\mathrm{N})$ (Ozaki et al. 2005) while it was enhanced due to codoping with iodine (I) donor impurities (Ozaki et al., 2006). In particular, for a fixed $\mathrm{Cr}$ content $x=0.05$, the FM transition temperature $T_{\mathrm{C}}$ increased up to $300 \mathrm{~K}$ with the codoping of iodine at a concentration of the order of $10^{18} \mathrm{~cm}^{-3}$, from $T_{\mathrm{C}} \sim 30 \mathrm{~K}$ in a layer without codoping (referred to as undoped sample). However, it was difficult to understand the realization of high-temperature ferromagnetism at a low $\mathrm{Cr}$ content, $x \simeq 0.05$, which is below the percolation limit of the zb structure, if we assume a shortrange interaction between $\mathrm{Cr}$ spins. Moreover, the combined analysis of the nanoscale chemical probing using TEM and the magnetization measurements with SQUID revealed that there is a systematic correlation between FM properties and the heterogeneity of the Cr distribution (Kuroda et al. 2007). In I-doped (Zn,Cr)Te crystals exhibiting ferromagnetism at RT, it was found that the $\mathrm{Cr}$ distribution is strongly inhomogeneous, i.e., nanoscale regions are formed, in which the concentration of $\mathrm{Cr}$ cations is high. According to the current understanding, in these Cr-rich volumes, $\mathrm{Cr}$ spins are ordered ferromagnetically due to the short distance between them. These FM clusters give rise to superparamagnetism controlled by the magnetic anisotropy of individual Cr-rich NCs and interactions among them.

The electronic structure of the $\mathrm{Cr}$ ions and host bands was investigated in $\mathrm{Zn}_{1-x} \mathrm{Cr}_{x} \mathrm{Te}(x=0.03$ and 0.15$)$ using XMCD and photoemission spectroscopy (Kobayashi et al. 2008). It was concluded that neither doubleexchange mechanism nor carrier-induced ferromagnetism are important, and the inhomogeneous distribution of $\mathrm{Cr}$ atoms dominantly influences the FM properties of $\mathrm{Zn}_{1-x} \mathrm{Cr}_{x} \mathrm{xTe}$.

In the case of $(\mathrm{Zn}, \mathrm{Cr}) \mathrm{Te}$, similar to other DMSs discussed, both chemical and crystallographic phase separations can be induced depending on $\mathrm{Cr}$ content and growth conditions (Kobayashi et al. 2012, Kuroda et al., 2007). The pairing energy of $\mathrm{Cr}$ ions, which is the driving force of the phase separation (see, Secs.II.B), is expected to vary with the $\mathrm{Cr}$ charge state (Dietl, 2006). Since the $\mathrm{Cr}$ $3 d$ electrons form deep donor and acceptor levels in the band gap of ZnTe (Godlewski and Kamińska, 1980), the studies of $(\mathrm{Zn}, \mathrm{Cr}) \mathrm{Te}$ demonstrated that the shift of the Fermi level produced by a change in the concentration of electrically active defects or shallow impurities alters the $\mathrm{Cr}$ charge state, which affects the Coulomb interaction between $\mathrm{Cr}$ ions and hence their aggregation (Kuroda et al. 2007).

In this section, we review recent experimental studies on spinodal nanodecomposition in $(\mathrm{Zn}, \mathrm{Cr}) \mathrm{Te}$ grown by MBE (Ishikawa et al., 2009, Kobayashi et al., 2012, Kuroda et al. 2007, Nishio et al., 2009) as well as asso- 
ciated magnetooptical (Kuroda et al., 2007, Ozaki et al. 2006) and magnetotransport properties (Kuroda et al., 2007). In the first part (Sec.VIII.B) we describe the properties of $(\mathrm{Zn}, \mathrm{Cr}) \mathrm{Te}$ films grown in the [001] crystallographic direction under the standard condition of MBE growth for the host binary compound $\mathrm{ZnTe}$ - at a substrate temperature of $T_{\mathrm{g}}=300^{\circ} \mathrm{C}$ and at a fixed growth rate of $\sim 1 \AA /$ sec. We also discuss the effect of codoping by I donors and $\mathrm{N}$ acceptors as well as varying the stoichiometry by changing the ratio of $\mathrm{Zn}$ and Te fluxes. The influence of the growth rate and temperature on the structural and magnetic properties of $(\mathrm{Zn}, \mathrm{Cr}) \mathrm{Te}: \mathrm{I}$ is presented in Sec.VIII.C. The accumulated data demonstrate the possibility of controlling the $\mathrm{Cr}$ aggregation by growth conditions and codoping with shallow impurities, corroborating the theoretical considerations on the pairing energy presented in Sec.II.B, as well as provide experimental illustration of the dairiseki and konbu phases discussed theoretically in Secs.II.C.1 and II.C.2. This control over the size and shape of Cr-rich regions substantiates the prospects of spinodal nanotechnology discussed in Sec.IX.

\section{B. Chemical phase separation and its control by the Fermi level position}

\section{Visualization of $\mathrm{Cr}$ distribution}

Figure 67 shows the EDS mapping images of the local $\mathrm{Cr}$ composition in I-doped, undoped, and N-doped $\mathrm{Zn}_{1-x} \mathrm{Cr}_{x}$ Te films with relatively low and high values of the average $\mathrm{Cr}$ content $x \simeq 0.05$ and 0.2 . In the case of the low $\mathrm{Cr}$ composition $x \simeq 0.05$, the $\mathrm{Cr}$ mapping images exhibit clear differences in the uniformity of $\mathrm{Cr}$ distribution between particular samples (Kuroda et al. 2007): uniform distributions within the spatial resolution of $2-3 \mathrm{~nm}$ in the undoped films grown under Te-rich flux [Fig.67(a)] and in the N-doped film [Fig.67(d)] versus nonuniform distributions in the undoped film grown under Zn-rich flux [Fig.67(b)] and in the I-doped film [Fig. 67(c)]. In the case of nonuniform distributions, the typical length scale of the compositional fluctuation is estimated to be $20-50 \mathrm{~nm}$. According to the spot analysis of the EDS spectra, a typical value of the local $\mathrm{Cr}$ composition inside the Cr-rich regions is $x \sim 0.1$.

The TEM observation of the series of the films with the $\mathrm{Cr}$ composition $x \simeq 0.05$ revealed that the structural properties are not much affected by the codoping of iodine or nitrogen, or the growth under different $\mathrm{Zn} / \mathrm{Te}$ flux ratios. As a typical example of the series of the films, the result of the I-doped film is shown in Fig.68. The TEM image in Fig.68(a) exhibits mostly zb crystalline structure, but there appear many stacking faults along the $\{111\}$ planes. It was suggested that strain associated with a preferential formation of TM cation dimers along the [110] direction accounts for the appearance of such faults during the growth (Birowska et al., 2012).
In addition, the electron diffraction from regions close to the bottom [Fig. 68(b)] and the top [Fig.68(c)] of the $\mathrm{Zn}_{1-x} \mathrm{Cr}_{x}$ Te layer exhibits different images, indicating the deterioration of crystallinity as the growth proceeds. In the diffraction image close to the bottom [Fig. 68(b)], there appear additional weak spots at one-third positions between the fundamental spots of a hexagonal arrangement of the zb structure, corresponding to a triplet periodicity in the stacking sequence along the $\{111\}$ planes in the observed stacking faults. On the other hand, the diffraction image close to the film top [Fig.68(c)] shows hexagonal patterns revealing the presence of zb nanocrystals with different crystallographic orientations at the end of the growth process. However, any apparent precipitates of other crystal structures were detected neither in the lattice image nor in the diffraction pattern.
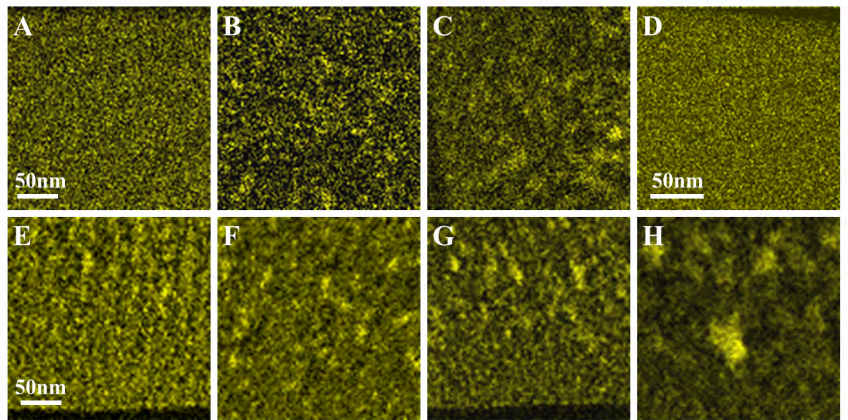

FIG. 67 (Color online) Mapping images of the emission intensity of the EDS $\mathrm{Cr} \mathrm{K}_{\alpha}$ line for cross-sectional pieces of $\mathrm{Zn}_{1-x} \mathrm{Cr}_{x}$ Te films with average $\mathrm{Cr}$ contents (a)-(d) $x \simeq 0.05$ and (e)-(h) $x \simeq 0.2$. (a), (e): undoped films grown under Te-rich flux; (b), (f): undoped films grown under Zn-rich flux; (c), (g), (h): I-doped films with iodine concentrations of $(\mathrm{c}),(\mathrm{g}) \sim 2 \times 10^{18} \mathrm{~cm}^{-3}$ and $(\mathrm{h}) \sim 1 \times 10^{19} \mathrm{~cm}^{-3} ;$ (d): Ndoped film with a nitrogen concentration of $\sim 2 \times 10^{20} \mathrm{~cm}^{-3}$. All these films were grown in the [001] crystallographic direction using $\mathrm{GaAs}(001)$ substrates under the standard condition of the MBE growth for $\mathrm{ZnTe}$ (the substrate temperature $T_{g}=300^{\circ} \mathrm{C}$ and the growth rate around $\left.1 \AA / \mathrm{sec}\right)$. Adapted from Kuroda et al. 2007 and Ishikawa et al. 2009.

For the high Cr composition of $x \sim 0.2$, the Cr distribution is always non-uniform, irrelevant of the codoping or the $\mathrm{Zn} / \mathrm{Te}$ flux ratio, but the degree of inhomogeneity changes depending on the codoping or the $\mathrm{Zn} / \mathrm{Te}$ flux ratio (Ishikawa et al. 2009). As shown in Fig.67(e)-(h), the inhomogeneity of Cr distribution in the undoped film grown under Te-rich flux is not significant, whereas the Cr distribution in the I-doped films and the undoped film grown under Zn-rich flux is strongly inhomogeneous. In particular, the size of $\mathrm{Cr}$-rich regions becomes larger in the I-doped film with a higher iodine concentration of $\sim 1 \times 10^{19} \mathrm{~cm}^{-3}$ [Fig. 67(h)]. A typical length scale of the Cr-rich regions is estimated to be $30-70 \mathrm{~nm}$. According to the EDS spot analysis, a maximum value of the local Cr composition in the Cr-rich regions is $x=0.4-0.5$. It is concluded from these results that the inhomogeneity 

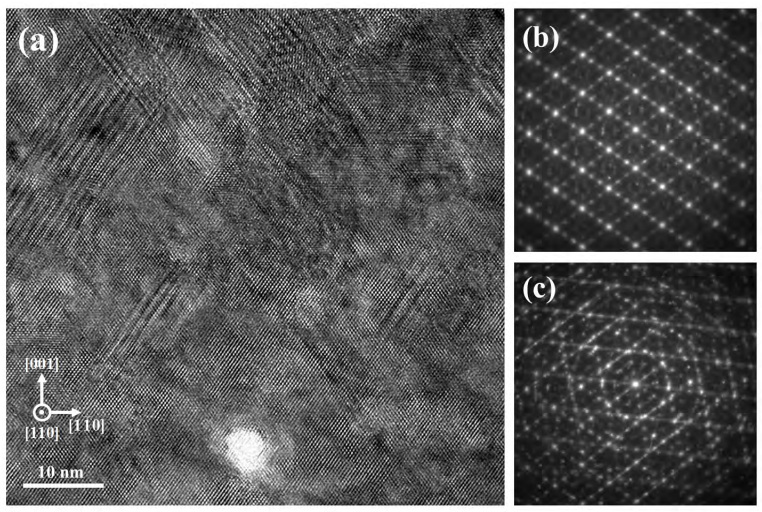

FIG. 68 TEM and electron diffraction images of the I-doped $\mathrm{Zn}_{0.95} \mathrm{Cr}_{0.05}$ Te film [sample (a) in Fig.67). (a) Cross-sectional lattice image. (b) Electron diffraction image from a region close to the interface with the ZnTe buffer layer and (c) from a region close to the surface. From Kuroda et al. 2007

of $\mathrm{Cr}$ distribution is enhanced either by codoping with iodine or by the growth under the Zn-rich condition for both low and high $\mathrm{Cr}$ contents.

\section{Superparamagnetic behavior due to the $\mathrm{Cr}$ aggregation}

The measurements of the temperature and magneticfield dependences of magnetization $M(T, H)$ using SQUID revealed that the magnetic properties also depend significantly on the $\mathrm{Zn} / \mathrm{Te}$ flux ratio or codoping with donor or acceptor impurities (Ishikawa et al. 2009, Kuroda et al., 2007). In Fig. 69, the temperature dependence of magnetization is compared for undoped films grown under Te-rich flux and I-doped films. As seen, superparamagnetic features, such as the irreversibility between magnetizations determined under FC and ZFC conditions, and a cusp in the $M(T)$ dependence taken in the ZFC process, are more pronounced in the case of the I-doped films than for the undoped films grown under Te-rich flux. The data in Fig. 69(a) indicate that superparamagnetic features appear at low temperatures also for the $\mathrm{Zn}_{0.95} \mathrm{Cr}_{0.05} \mathrm{Te}$ film for which the Cr distribution is uniform according to Fig.67(a). This means that $\mathrm{Cr}$ aggregates with diameters below spatial resolution of EDS $(2-3 \mathrm{~nm})$ are present in such samples. The magnitude of blocking temperature $T_{\mathrm{b}}$, i.e., a temperature value corresponding to the maximum in the $M(T)$ dependence taken in the ZFC process, is much higher for the I-doped films than in the case of undoped films grown in the Te-rich flux. The paramagnetic Curie-Weiss temperature $\theta_{P}$ obtained from the linear fitting of the temperature dependence of the inverse magnetic susceptibility $\chi(T)$ is also higher in the case of the I-doped films compared to the undoped films grown under Te-rich flux.

The magnitudes of three characteristic temperatures, $T_{\mathrm{b}}, \theta_{P}$, and the apparent Curie temperature $T_{\mathrm{C}}^{(\mathrm{app})}$ de- duced from the Arrott plot analysis of the $M(H)$ dependence, are plotted in Fig.70 for samples with the average Cr contents $x \simeq 0.05$ and 0.2 as a function of the iodine concentration and the $\mathrm{Te} / \mathrm{Zn}$ flux ratio. In the case of $x \simeq 0.05$, these temperatures are closely correlated with the uniformity of the $\mathrm{Cr}$ distribution: $T_{\mathrm{C}}^{(\mathrm{app})}, T_{\mathrm{b}}$, and $\theta_{P}$ assume maximum values at the iodine concentration $\sim 2 \times 10^{18} \mathrm{~cm}^{-3}$ for the I-doped films and $\mathrm{Te} / \mathrm{Zn}$ flux ratio 0.7 (Zn-rich flux) for the undoped films, which correspond to the most pronounced inhomogeneity of the $\mathrm{Cr}$ distribution. On the other hand, in the case of $x \sim 0.2$, $T_{\mathrm{C}}^{(\mathrm{app})}$ and $\theta_{P}$ are already high even in the undoped film grown under Te-rich flux and do not increase much for the growth under Zn-rich flux or the codoping of iodine. In contrast, $T_{\mathrm{b}}$ shows a sizable change depending on the flux ratio or the I doping; in the undoped films, $T_{\mathrm{b}}$ increases with the decrease of the $\mathrm{Te} / \mathrm{Zn}$ flux ratio, and in the I-doped films, $T_{\mathrm{b}}$ increases with the increase of iodine concentration at first and reaches a maximum at $\sim 5 \times 10^{18} \mathrm{~cm}^{-3}$, and then decreases gradually with a further increase of iodine content. These findings indicate that the magnitude of $T_{\mathrm{b}}$ shows a close correlation with the degree of inhomogeneity in the $\mathrm{Cr}$ distribution at high $\mathrm{Cr}$ compositions.

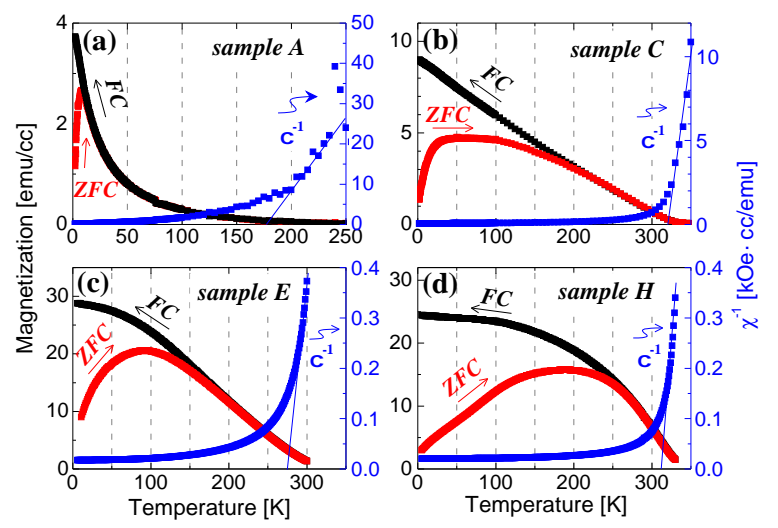

FIG. 69 (Color online) Temperature dependence of magnetization and the inverse magnetic susceptibility of $\mathrm{Zn}_{1-x} \mathrm{Cr}_{x} \mathrm{Te}$ films: (a) undoped $\mathrm{Zn}_{0.95} \mathrm{Cr}_{0.05} \mathrm{Te}$ film grown under Te-rich flux (sample A in Fig 67); (b) I-doped $\mathrm{Zn}_{0.95} \mathrm{Cr}_{0.05} \mathrm{Te}$ film (sample C); (c) undoped $\mathrm{Zn}_{0.81} \mathrm{Cr}_{0.19}$ Te film grown under Terich flux (sample E); (d) I-doped $\mathrm{Zn}_{0.81} \mathrm{Cr}_{0.19}$ Te film (sample H). Adapted from Kuroda et al. 2007.

These experimental observations demonstrate that the magnetic properties of $(\mathrm{Zn}, \mathrm{Cr}) \mathrm{Te}$ films are closely correlated with the uniformity of $\mathrm{Cr}$ distribution. In particular, non-uniform $\mathrm{Cr}$ distributions give rise to higher values of characteristic temperatures introduced above. Since the FM exchange interaction between $\mathrm{Cr}$ spins is considered to be of short-range (Bergqvist et al., 2004), the long-range FM order cannot be expected for average $\mathrm{Cr}$ compositions below the percolation limit for the nearest-neighbor coupling in the fcc lattice, $x<0.20$, if we assume a random distribution of $\mathrm{Cr}$ ions. On the other 


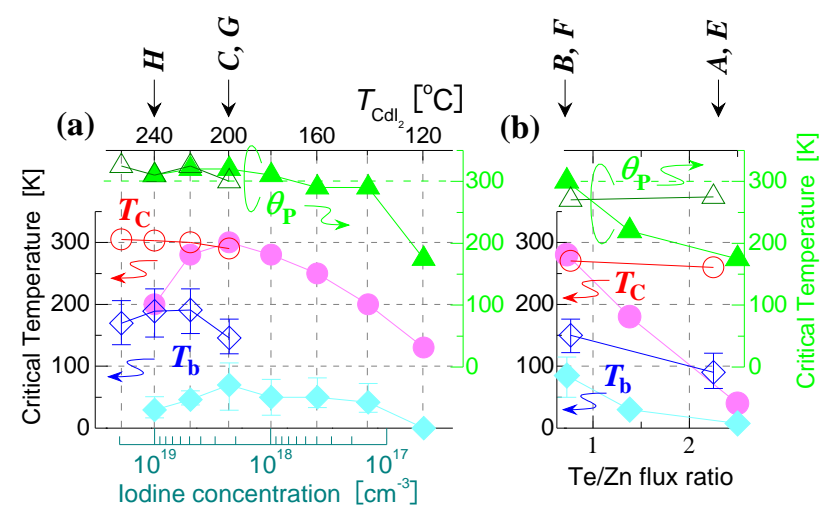

FIG. 70 (Color online) Three characteristic temperatures for I-doped and undoped $\mathrm{Zn}_{1-x} \mathrm{Cr}_{x}$ Te films with $x \simeq 0.05$ and $x \sim 0.2$ : the apparent Curie temperature $T_{\mathrm{C}}^{(\text {app })}$ (circles), the paramagnetic Curie-Weiss temperature $\theta_{P}$ (triangles) and the blocking temperature $T_{\mathrm{b}}$ (diamonds) are plotted as a function of (a) the iodine concentration for I-doped films and (b) the $\mathrm{Te} / \mathrm{Zn}$ flux ratio for undoped films. For clarity, the vertical scale for $\theta_{P}$ (right) is shifted from that for $T_{\mathrm{C}}^{(\mathrm{app})}$ and $T_{\mathrm{b}}$ (left). The data for $x \simeq 0.05$ and 0.2 are represented by full and open symbols, respectively. The labels $\mathrm{A}-\mathrm{H}$ above the figure serve to identify the films shown in Fig.67. From Ishikawa et al. 2009

hand, if the alloy is phase-separated into regions with low and high $\mathrm{Cr}$ contents, Cr spins inside the Cr-rich regions can order ferromagnetically, so that the crystal containing these FM nanoclusters is expected to exhibit superparamagnetic features. Below the blocking temperature $T_{\mathrm{b}}$, whose magnitude is given by the product of the mean cluster volume $V$ and the density of the magnetic anisotropy energy $K$, FM-like properties are observed in superparamagnetic systems, including magnetization hysteresis and remanence. A correlation between the values of $T_{\mathrm{b}}$ and the inhomogeneity of Cr distribution confirms this interpretation. In addition, rather broad peaks in the $M(T)$ curves obtained under the ZFC condition reflect a dispersion in values of blocking temperatures. The apparent Curie temperature $T_{\mathrm{C}}^{(\mathrm{app})}$, corresponding to temperature at which hysteretic behaviors due to the magnetic anisotropy of the clusters disappear entirely, is determined by an upper bound of the $T_{\mathrm{b}}$ distribution. On the other hand, the paramagnetic Curie-Weiss temperature $\theta_{P}$, deduced from the fitting of the linear dependence in the $\chi^{-1}(T)$ curves in the high-temperature range, is determined by interactions between $\mathrm{Cr}$ spins inside the Cr-rich NCs and, therefore, is virtually independent of their volume $V$. This is consistent with the observation that $\theta_{P}$ is less dependent on the uniformity of the $\mathrm{Cr}$ distribution than $T_{\mathrm{C}}^{(\mathrm{app})}$ and $T_{\mathrm{b}}$.

Altogether, the data imply that zb Cr-rich $(\mathrm{Cr}, \mathrm{Zn}) \mathrm{Te}$ with the $\mathrm{ZnTe}$ lattice parameter is characterized by $T_{\mathrm{C}} \simeq$ $300 \mathrm{~K}$. A ferromagnetic ground state was theoretically predicted for such zb-CrTe (Zhao and Zunger, 2005).

\section{Mechanism of $\mathrm{Cr}$ aggregation}

Similarly to the most combinations of magnetic elements and host semiconductors, the solubility of $\mathrm{Cr}$ in $\mathrm{ZnTe}$ is low and the incorporation of $\mathrm{Cr}$ beyond a certain limit results in the crystallographic or chemical phase separation. The driving force of the phase separation is an attractive interaction between magnetic cations. The key to understanding the observation that the uniformity of $\mathrm{Cr}$ distribution varies with the $\mathrm{Zn} / \mathrm{Te}$ flux ratio or the codoping of donor or acceptor impurities is the dependence of the attractive interaction on the Fermi level position within the band gap (Dietl, 2006, Ye and Freeman, 2006). In the case of intrinsic ZnTe, Cr assumes the 2+ charge state. However, this charge state can be changed by trapping a hole or an electron since the substitutional $\mathrm{Cr}$ forms the deep donor $\mathrm{Cr}^{2+/ 3+}$ and acceptor $\mathrm{Cr}^{1+/ 2+}$ levels within the band gap of the host ZnTe (Godlewski and Kamińska, 1980). Therefore, the codoping with shallow impurities changes the $\mathrm{Cr}$ valence and, thus, the $\mathrm{Cr}$ $\mathrm{Cr}$ interaction. In particular, an additional Coulomb repulsion between those Cr ions that have trapped a carrier hinders the $\mathrm{Cr}$ aggregation, leading to a uniform distribution of $\mathrm{Cr}$ cations. This is in contrast to a non-uniform distribution driven by the attractive interaction between isoelectronic $\mathrm{Cr}^{2+}$ ions, associated with the contribution of TM $3 d$ states to bonding, as discussed in Sec.II.B.

In order to understand the observed phenomena based on this model, one should teke into account that $\mathrm{ZnTe}$ crystals, due to the native formation of $\mathrm{Zn}$ vacancies, have a tendency to become $p$-type even without intentional doping (Baron et al., 1998). In (Zn,Cr)Te films grown under the Te-rich condition, the $\mathrm{Zn}$ vacancies are formed, so that a part of the $\mathrm{Cr}$ ions assumes the $\mathrm{Cr}^{3+}$ charge state. The growth under the Zn-rich condition or codoping with iodine donors restore the isoelectronic $\mathrm{Cr}^{2+}$ configuration, as the $\mathrm{Zn}$ vacancies are suppressed by a surplus supply of $\mathrm{Zn}$ or compensated by the I donors. In the I-doped films, the degree of inhomogeneity and the value of the blocking temperature $T_{\mathrm{b}}$ (and also $T_{\mathrm{C}}^{(\mathrm{app})}$ for $x \simeq 0.05$ ) attain a maximum when the iodine and $\mathrm{Zn}$ vacancy concentrations become equal, so that the charge state of all $\mathrm{Cr}$ ions is $2+$. In contrast, codoping with $\mathrm{N}$ increases the concentration of $\mathrm{Cr}^{3+}$ ions (as observed by XMCD (Yamazaki et al. 2011)), which diminishes attractive forces between $\mathrm{Cr}$ cations and results in their uncorrelated distribution.

\section{Magnetooptical and magnetotransport properties}

Magnetooptical properties were investigated for crystals exhibiting a high-temperature ferromagnetism assigned, as explained previously, to a nonuniform $\mathrm{Cr}$ distribution. Figure 71 shows the result of the MCD mea- 
surement on an I-doped $\mathrm{Zn}_{1-x} \mathrm{Cr}_{x}$ Te film with a relatively low $\mathrm{Cr}$ content of $x=0.07$ and $T_{\mathrm{C}}^{(\mathrm{app})} \simeq 300 \mathrm{~K}$ (Ozaki et al., 2006). As shown in Fig.71(a), the MCD spectrum exhibits a broad band below the band-gap energy $2.38 \mathrm{eV}$ of ZnTe, in contrast to a sharp peak in the spectrum of ZnTe. According to Fig. 71(b), the MCD intensity at a fixed photon energy shows virtually identical magnetic-field dependence as magnetization measured by SQUID. These features of MCD were also observed in undoped films $x=0.07$ grown under Te-rich flux (Kuroda et al. , 2005), in which smaller scale inhomogeneities in the $\mathrm{Cr}$ distribution lead to lower value of $T_{\mathrm{C}}^{(\mathrm{app})}$, of the order of $100 \mathrm{~K}$.
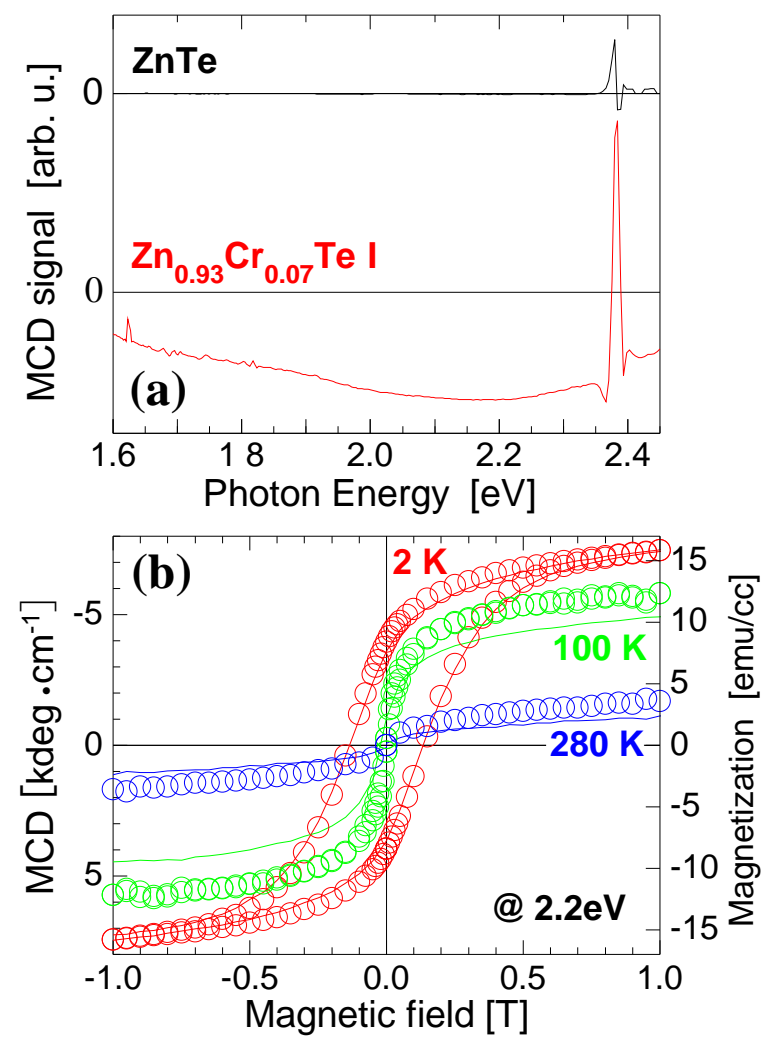

FIG. 71 (Color online) Results of MCD studies on an I-doped $\mathrm{Zn}_{0.93} \mathrm{Cr}_{0.07} \mathrm{Te}$ film. The measurement was performed in the transmission mode in the magnetic field perpendicular to the film plane (Faraday configuration). (a) The MCD spectra at $2 \mathrm{~K}$ and $1 \mathrm{~T}$, with the reference data for a $\mathrm{ZnTe}$ film. (b) Magnetic-field dependence of the MCD intensities at a photon energy of $2.2 \mathrm{eV}$ (circles), together with magnetization values measured by SQUID (lines). From Ozaki et al., 2006

The broadband in the MCD spectrum could be attributed to optical transitions from the valence band to the $\mathrm{Cr} 3 d$ level in the middle of the band gap, with a significant broadening of the absorption energy due to the lattice relaxation (Godlewski and Kamińska, 1980) or may originate from the presence of Cr-rich regions that affect MCD in a wide spectral region via magnetization- dependent boundary conditions for light propagation (the Kerr effect). The consistency between magnetooptical and magnetization data, observed also in the case of decomposed (Ga,Mn)As, (Ge,Mn), and (Ge,Fe), as discussed in Secs.III.C, VI.B.1, and VII.D, respectively, suggests that in these systems magnetooptical response is dominated by regions with high TM concentrations, which account for the FM properties.

In addition, the anomalous Hall effect was observed in an I-doped $\mathrm{Zn}_{1-x} \mathrm{Cr}_{x}$ Te film (Kuroda et al., 2007, Supplementary Information). The observation of the magnetooptical and magnetotransport properties peculiar to DMSs even in phase-separated (Zn,Cr)Te layers suggests a possibility of new functionalities in hybrid structure consisting of TM-rich nanocrystals embedded in a semiconductor matrix, as discussed in Sec. IX.

\section{Crystallographic phase separation and konbu phase}

\section{Structural nanocharacterization}

Structural, compositional, and magnetic properties were investigated for a series of I-doped $\mathrm{Zn}_{1-x} \mathrm{Cr}_{x}$ Te films varying substrate temperature during the growth, growth rate, and crystallographic orientation of the substrate (Nishio et al. , 2009). It was found that the substrate temperature is a critical factor controlling the phase separation. For the lower average $\mathrm{Cr}$ content $x \simeq 0.05$, the structural properties of I-doped $\mathrm{Zn}_{1-x} \mathrm{Cr}_{x} \mathrm{Te}$ films change with the substrate temperature $T_{\mathrm{g}}$, while the uniformity of $\mathrm{Cr}$ distribution is not much different. Figure 72 shows the results of TEM and EDS studies of I-doped $\mathrm{Zn}_{1-x} \mathrm{Cr}_{x}$ Te $(x \simeq 0.05)$ films grown in the [001] direction at various values of $T_{\mathrm{g}}$. As shown in the TEM and diffraction images (left column), the crystallinity exhibits a marked variation with $T_{\mathrm{g}}$; at an intermediate temperature of $T_{\mathrm{g}}=270^{\circ} \mathrm{C}$, the crystal consists dominantly of the $\mathrm{zb}$ structure, but the stacking faults along the $\{111\}$ planes are observed in the lattice image and additional spots corresponding to the triplet periodicity of the stacking faults appear in the diffraction image. The crystallinity is deteriorated by decreasing $T_{\mathrm{g}}$ down to $240^{\circ} \mathrm{C}$, exhibiting polycrystalline features in addition to the stacking faults, while it looks much improved by increasing $T_{\mathrm{g}}$ up to $360^{\circ} \mathrm{C}$, the TEM and diffraction images exhibiting almost perfect zb structure without the stacking faults. On the other hand, as shown in the $\mathrm{Cr}$ mapping images (right column), the $\mathrm{Cr}$ distribution is inhomogeneous in all the films, but the degree of inhomogeneity seems to be slightly reduced with the increase of $T_{\mathrm{g}}$. The results of XAS measurements at $\mathrm{Cr} K$-edge for the same series of I-doped $\mathrm{Zn}_{1-x} \mathrm{Cr}_{x} \mathrm{Te}(x \simeq 0.05)$ films suggested a change in the local crystallographic structure depending on the substrate temperature (Ofuchi et al. 2009), which is correlated with the results of the TEM observation.

A natural explanation of these results would be that 

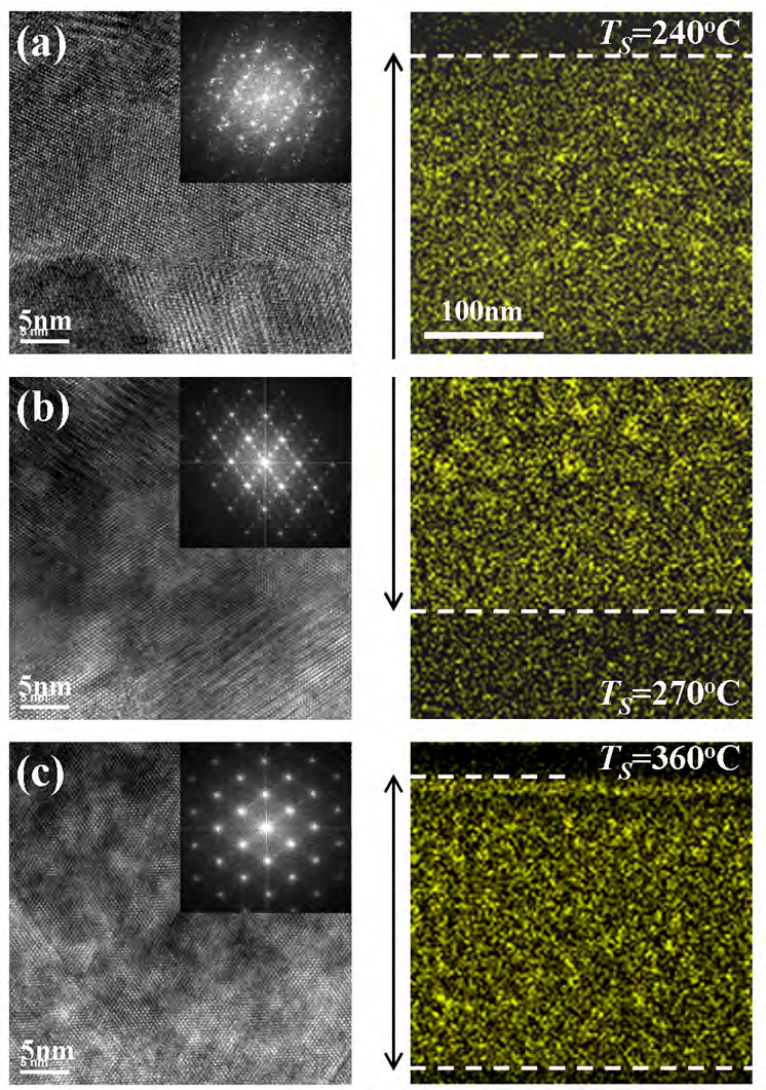

FIG. 72 (Color online) TEM and diffraction images (left) and EDS mapping images of Cr (right) for cross-sectional pieces of I-doped $\mathrm{Zn}_{1-x} \mathrm{Cr}_{x}$ Te films $(x \simeq 0.05)$ grown in the [001] direction at various substrate temperatures. (a) $T_{\mathrm{g}}=240^{\circ} \mathrm{C}$; (b) $T_{\mathrm{g}}=270^{\circ} \mathrm{C}$; (c) $T_{\mathrm{g}}=360^{\circ} \mathrm{C}$. In the EDS mapping images, the range of the $\mathrm{Zn}_{1-x} \mathrm{Cr}_{x}$ Te:I layer and the boundary with the ZnTe buffer layer are indicated by arrows and dashed lines, respectively. From Nishio et al. 2009.

lattice defects appearing at low $T_{\mathrm{g}}$ lower kinetic barriers for $\mathrm{Cr}$ diffusion, so that the $\mathrm{Cr}$ aggregation is efficient even at low $T_{\mathrm{g}}$.

In contrast, for the higher average $\mathrm{Cr}$ content $x \sim 0.2$, the substrate temperature significantly affects the form of the phase separation (Nishio et al. 2009). Figure 73 presents $\mathrm{Cr}$ mapping images of the films grown onto either (001) or (111) substrates at intermediate and high substrate temperatures, $T_{\mathrm{g}}=300$ and $360^{\circ} \mathrm{C}$. As shown in Fig. 73(a), the Cr-rich regions form isolated NCs at $T_{\mathrm{g}}=300^{\circ} \mathrm{C}$, whereas according to Figs. $73(\mathrm{~b}, \mathrm{c})$, in the case of films grown at $T_{\mathrm{g}}=360^{\circ} \mathrm{C}$, Cr-rich regions look like continuous nanocolumns aligned approximately along the $\langle 111\rangle$ direction of the host crystal. As estimated by the EDS spot analysis, a maximum value of the local Cr composition is $x=0.4-0.5$, in the case of Cr-rich both NCs and nanocolumns.

Figure 74 shows TEM and EELS images allowing to shed some light on the crystal structure of Cr-rich regions in an I-doped $\mathrm{Zn}_{1-x} \mathrm{Cr}_{x}$ Te films $(x=0.25)$ grown
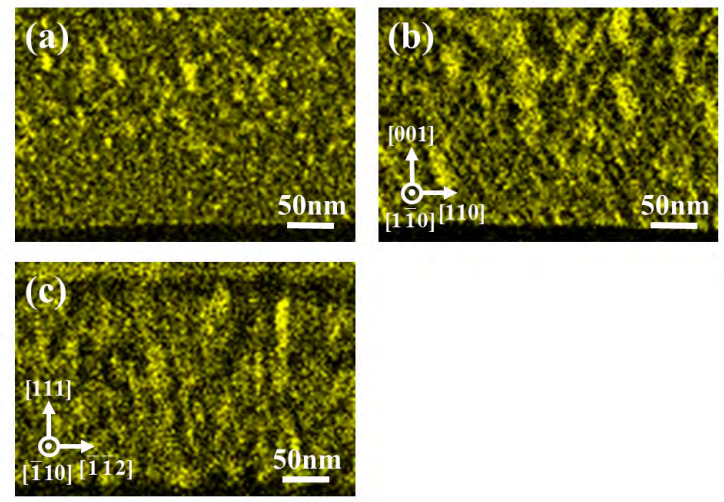

FIG. 73 (Color online) Cross-sectional EDS mapping images of Cr for $\mathrm{Zn}_{1-x} \mathrm{Cr}_{x}$ Te:I films $(x \simeq 0.25)$ grown (a) at a substrate temperature $T_{\mathrm{g}}=300^{\circ} \mathrm{C}$ in the [001] direction, (b) at $T_{\mathrm{g}}=360^{\circ} \mathrm{C}$ in the $[001]$ direction, and (c) at $T_{\mathrm{g}}=360^{\circ} \mathrm{C}$ in the [111] direction, imposed by crystallographic orientations of GaAs substrates. Cr-rich regions are formed as isolated NCs in (a), while they are elongated and form continuous nanocolumns in (b), (c). From Nishio et al. 2009.

at $T_{\mathrm{g}}=360^{\circ} \mathrm{C}($ Kobayashi et al., 2012$)$. In the TEM image in Fig.74(a), moiré fringes appear in many regions, which suggests that these regions are composed of a mixed phase with different crystal structures, pointing to the presence of crystallographic phase separation. By comparing the TEM image [Fig. 74(a)] with the EELS image [Fig. $74(\mathrm{~b})]$ of the same area, it is confirmed that the moiré regions in the TEM image correspond to the regions with a high value of $\mathrm{Cr}$ content. In a magnified lattice image in Fig. 74(c), the moiré region is boarded by the two $\{111\}_{z b}$ planes, and there appears another region exhibiting a different structure, identified as a hexagonal structure from the arrangement of diffraction spots in the fast Fourier transform image shown in Fig.74(d). The lattice parameters deduced from the spacing between the spots are $c=6.32 \AA$ and $a=4.18 \AA$, close to the values reported for bulk CrTe in the NiAs structure (Dijkstra et al., 1989, Ohta et al., 1993). This suggests that the precipitates consist of NiAs-type CrTe or of nonstoichiometric $\mathrm{Cr}_{1-\delta} \mathrm{Te}$ in a hexagonal structure. According to the TEM and diffraction images in Fig.74, the $c$-axis of elongated hexagonal NCs is nearly parallel to the $\{111\}_{\mathrm{zb}}$ planes of the host. In the case of $T_{\mathrm{g}}=360^{\circ} \mathrm{C}$, a $3 \mathrm{D}$ analysis using atom probe tomography revealed that the Cr-aggregated regions were formed as thin plates with the base plane nearly parallel to the $\{111\}_{\text {zb }}$ planes (Kodzuka, 2012).

These observations of the crystallographic phase separation support theoretical computations indicating that zb-CrTe with the lattice parameter of $\mathrm{ZnTe}$ is unstable against the formation of CrTe in the NiAs crystal structure (Zhao and Zunger, 2005).

In the conventional $\omega-2 \theta$ XRD scan, which probes XRD from the film plane, only maxima correspond- 

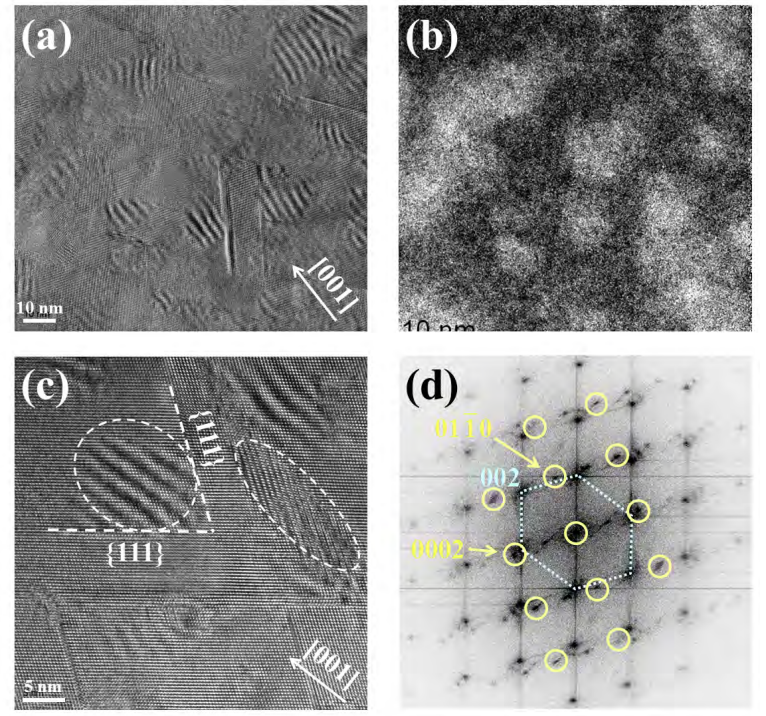

FIG. 74 (Color online) TEM and EELS images of the Idoped $\mathrm{Zn}_{0.75} \mathrm{Cr}_{0.25} \mathrm{Te}$ film grown in the [001] direction at $T_{\mathrm{g}}=360^{\circ} \mathrm{C}$ [the same film as shown in Fig.73(b)] giving evidence for crystallographic phase separations. (a) TEM image. (b) Cr mapping image of the same area obtained by EELS. (c) Lattice image in a magnified scale. A moiré region boarded by the two $\{111\}_{\mathrm{zb}}$ planes and a region of a different crystallographic structure are observed (encircled by broken lines). (d) FFT image determined from the data in (c). The set of diffraction spots of a hexagonal arrangement (connected by dotted line) originates from the zb structure and that of a rectangular arrangement (denoted by circles) originates from the hexagonal structure whose $c$-plane is almost parallel to the $\{111\}_{\mathrm{zb}}$ planes. From Kobayashi et al. 2012.

ing to the zb host crystal were detected for the (001) $\mathrm{Zn}_{1-x} \mathrm{Cr}_{x}$ Te:I films with $x \sim 0.2$. However, hexagonal $\mathrm{Cr}_{1-\delta} \mathrm{Te}$ precipitates that have principal diffraction planes inclined against the film plane, can be detected in the $\omega$ scan, as shown in Fig.75. This measurement was performed with the incident and reflected $\mathrm{x}$ rays in the (110) plane of the host zb structure. By locking $2 \theta$ to the values corresponding to the (1101) and (1102) planes of the hexagonal $\mathrm{Cr}_{1-\delta} \mathrm{Te}$, the diffractions from these planes were detected in an almost symmetric way with respect to $\omega$. The values of $\omega$ of the diffraction peaks are close to the ones expected from the crystallographic relation of these NCs to the host crystal revealed by the TEM observation. On the other hand, in the same $\omega$-scan measurement in another configuration with the incident and reflected $x$ rays in the $(110)_{\mathrm{zb}}$ plane, the intensity of the diffraction from the hexagonal nanocrystals was much reduced (Kobayashi et al. 2013). This result suggests that the hexagonal $\mathrm{Cr}_{1-\delta} \mathrm{Te}$ tends to stack preferentially on the (111) zb plane [or Zn-terminated (111) $A$ plane] rather than $(\overline{1} \overline{1} \overline{1})_{z b}$ plane [or Te-terminated (111) $B$ plane]. This preference could be understood from the arrangement of atoms on the interface between the hexagonal $\mathrm{Cr}_{1-\delta} \mathrm{Te}$ and the host zb crystals.

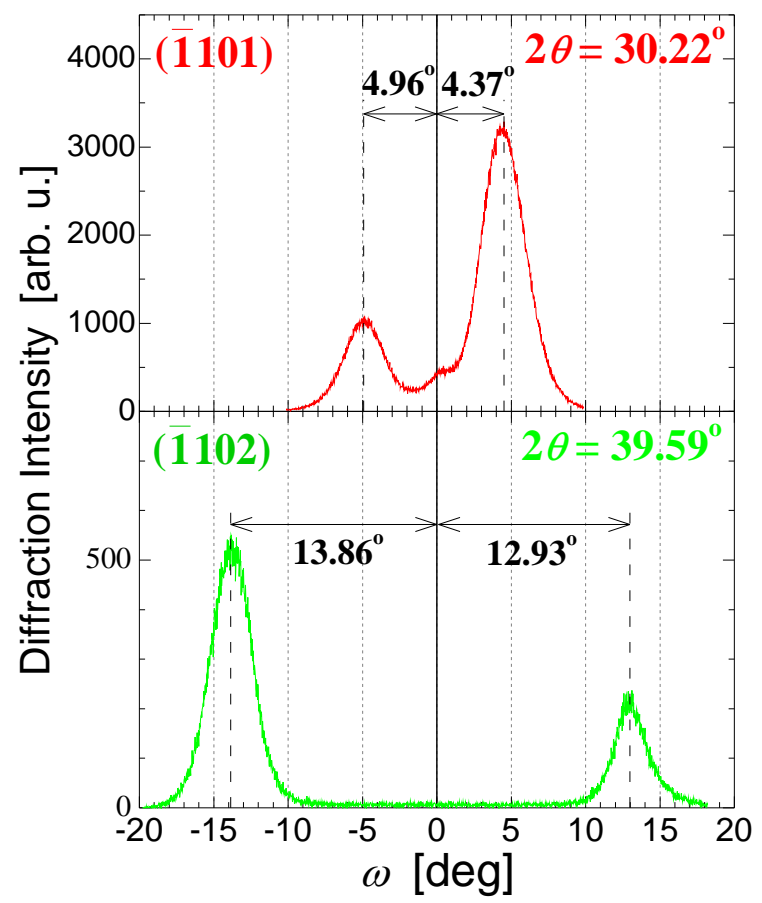

FIG. 75 (Color online)XRD $\omega$-scan profiles of an I-doped $\mathrm{Zn}_{0.73} \mathrm{Cr}_{0.27} \mathrm{Te}$ film grown in the [001] orientation at $T_{\mathrm{g}}=$ $390^{\circ} \mathrm{C}$. The diffraction profiles from the plane $\left(2 \theta=30.22^{\circ}\right)$ (upper panel) and the plane $\left(2 \theta=39.59^{\circ}\right.$ ) (lower panel) are plotted, respectively. This measurement was performed in the configuration with the incident and reflected x-ray in the (110) plane of the host zb structure. From Kobayashi et al. 2012

The intensity of the diffraction from the hexagonal $\mathrm{Cr}_{1-\delta} \mathrm{Te}$ gives us an estimate of the amount of the precipitates formed in the crystal. The $\omega$-scan measurement on the series of $\mathrm{Zn}_{1-x} \mathrm{Cr}_{x}$ Te:I films grown at different substrate temperatures revealed how the amount of the precipitates depends on the growth temperature. As seen in Fig. 76 , in which the diffraction intensity from the (1101) plane of the hexagonal $\mathrm{Cr}_{1-\delta} \mathrm{Te}$ is plotted as a function of the substrate temperature $T_{\mathrm{g}}$, the hexagonal precipitates are formed in a larger quantity at a higher $T_{\mathrm{g}}$.

As described previously, it was revealed that the substrate temperature during the growth significantly affects the $\mathrm{Cr}$ aggregation; the $\mathrm{Cr}$-aggregated regions are formed in almost spherical NCs at a low $T_{\mathrm{g}}$ while they are formed in thin plates at higher $T_{\mathrm{g}}$. In addition, the amount of the hexagonal precipitates increases with the increase of $T_{\mathrm{g}}$. The observed variation in the shape of $\mathrm{Cr}$-aggregated regions with the substrate temperature reflects a difference in the dimensionality of the spinodal decomposition, which results in the formation of the dairiseki or konbu phase, as discussed in Secs.II.C.1 and II.C.2 According to a theoretical consideration (Fukushima et al. 2006b), the spinodal decomposition in the layer-by-layer growth mode results in the formation of one-dimensional colum- 


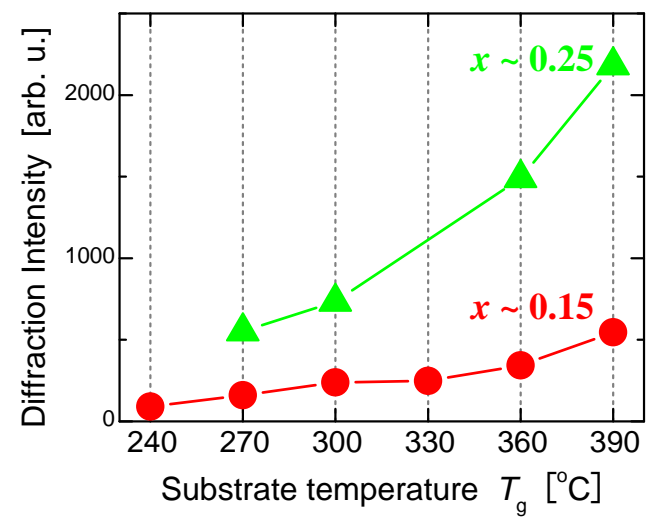

FIG. 76 (Color online) The diffraction intensity from the (1101) plane of the hexagonal $\mathrm{Cr}_{1-\delta}$ Te nanocrystals plotted as a function of the substrate temperature $T_{\mathrm{g}}$ for the two series of $\mathrm{Zn}_{1-x} \mathrm{Cr}_{x}$ Te:I films with $x \simeq 0.15$ and 0.25 . From Kuroda et al., unpublished.

nar regions with high content of the magnetic element. With an enhanced migration of impinging atoms on the growing surface at a higher substrate temperature, $\mathrm{Cr}$ atoms tend to aggregate in such places on the top surface where Cr-aggregated areas are already formed in the layer just below the growing surface. As a result, the Cr-rich regions form continuous nanocolumns, instead of isolated clusters appearing in the case of slow surface migration. Once Cr-aggregated regions are formed inside the crystal, they can transform at sufficiently high $T_{\mathrm{g}}$ into a stable $\mathrm{Cr}_{1-\delta} \mathrm{Te}$ hexagonal compound that assumes crystal orientation insuring the best match of the atom positions with the host, i.e., minimization of the interfacial energy.

\section{Magnetic properties}

Three characteristic temperatures $T_{\mathrm{C}}^{(\mathrm{app})}, \theta_{P}$, and $T_{\mathrm{b}}$ are presented in Fig. 77 as a function of $T_{\mathrm{g}}$ for the two series of I-doped $\mathrm{Zn}_{1-x} \mathrm{Cr}_{x}$ Te films with the low and high average $\mathrm{Cr}$ contents of $x \simeq 0.05$ (full symbols) and 0.25 (empty symbols). In the case of $x \simeq 0.05$, the film grown at the lowest $T_{\mathrm{g}}$ of $240^{\circ} \mathrm{C}$ did not exhibit ferromagnetism even at $2 \mathrm{~K}$, presumably due to the deterioration of crystallinity. In contrast, the films obtained at $T_{\mathrm{g}}$ from 270 to $390^{\circ} \mathrm{C}$ show high-temperature ferromagnetism, consistent with the presence of $\mathrm{Cr}$-rich regions. The observed values $T_{\mathrm{C}}^{(\mathrm{app})} \simeq 300 \mathrm{~K}$ and $\theta_{P} \simeq 330 \mathrm{~K}$ for films with $x \simeq 0.25$ are consistent with the fact that $\mathrm{Cr}_{1-\delta} \mathrm{Te}$ in bulk form exhibits ferromagnetism with $T_{\mathrm{C}}=325-360 \mathrm{~K}$ for a relatively a small amount of Cr deficiency (Shimada et al., 1996$)$, though $T_{\mathrm{C}}$ decreases when the amount of Cr deficiency increases further on (Ohta et al. 1993).
Furthermore, in agreement with TEM data pointing to smaller volumes of Cr-rich regions in the case of samples with lower $x$, the magnitudes of $T_{\mathrm{b}}$ are significantly lower for $x \simeq 0.05$ compared to the values found for $x \simeq 0.25$. However, rather surprisingly, $T_{\mathrm{C}}^{(\mathrm{app})}$ and $\theta_{P}$ tend to decrease from 300 down to $250 \mathrm{~K}$ with $T_{\mathrm{g}}$ for samples with $x \simeq 0.05$. This result points to a complex interplay between the degree of host crystallinity, TM aggregation, and resulting $T_{\mathrm{C}}$ of embedded NCs.

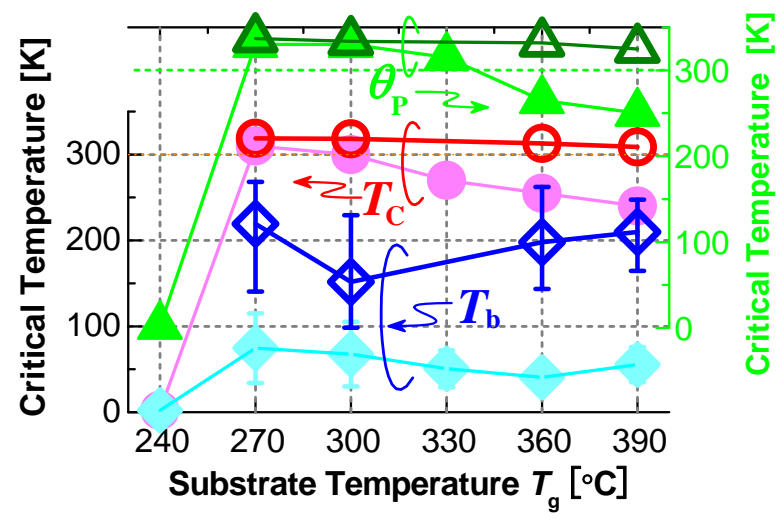

FIG. 77 (Color online) Plot of three characteristic temperatures $T_{\mathrm{C}}^{(\text {app })}$ (circles), $\theta_{P}$ (triangles), and $T_{\mathrm{b}}$ (diamonds) as a function of the substrate temperature $T_{\mathrm{g}}$ for I-doped $\mathrm{Zn}_{1-x} \mathrm{Cr}_{x}$ Te films with the low $(x \simeq 0.05)$ and high $(x \simeq 0.25)$ average Cr contents (full and empty symbols, respectively). From Kuroda et al., unpublished.

It can be expected that the strain and shape of NCs affect their magnetic anisotropy. Figure 78 presents magnetization loops $M(H)$ for magnetic fields perpendicular and parallel to the film plane for the I-doped (111) $\mathrm{Zn}_{1-x} \mathrm{Cr}_{x}$ Te film $(x=0.22)$, in which Cr-rich NCs show the elongated shape with long axes almost perpendicular to the film plane (Nishio et al. 2009). As seen, hysteretic behaviors are more pronounced (i.e., the coercive force is larger) for the perpendicular magnetic field. This suggests that the easy direction is parallel to the long axis of the NCs, the expected result for the shape-dependent direction of the demagnetization field and weak in-plane crystalline magnetic anisotropy.

\section{Summary}

The comprehensive studies of $(\mathrm{Zn}, \mathrm{Cr}) \mathrm{Te}$ described in this section show that the $\mathrm{Cr}$ distribution is affected significantly by codoping with donor or acceptor impurities or by the growth under different $\mathrm{Zn} / \mathrm{Te}$ flux ratios. This behavior originates from the influence of codoping and growth conditions upon the position of the Fermi level in the band gap, which controls the $\mathrm{Cr}$ charge state: from $\mathrm{Cr}^{+1}$ for high I donor concentrations to $\mathrm{Cr}^{3+}$ at high density of $\mathrm{N}$ acceptor impurities or $\mathrm{Zn}$ vacancies. These findings corroborate the influence of 

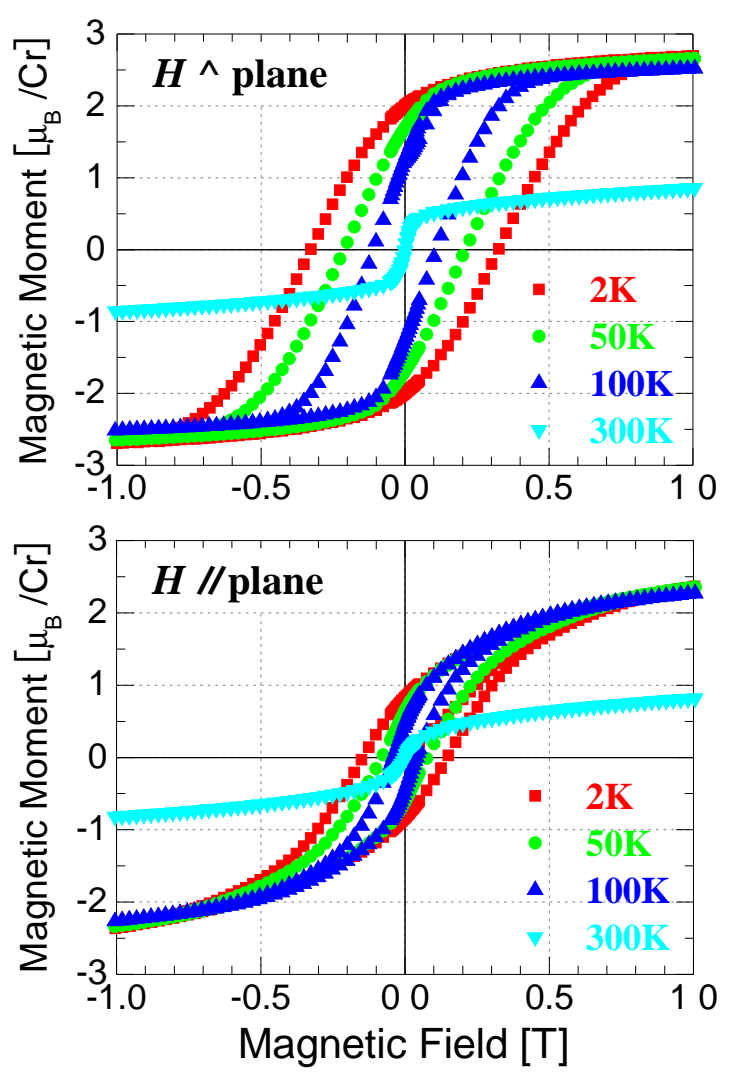

FIG. 78 (Color online) Magnetization loops in $\mathrm{Zn}_{0.78} \mathrm{Cr}_{0.22}$ Te:I film grown in the [111] direction at $T_{\mathrm{g}}=360^{\circ} \mathrm{C}$ [the same film as shown in Fig. 73 (c)]. The upper (lower) panel shows the results obtained for magnetic fields perpendicular (parallel) to the film plane. The coercive field $H_{c}$ for the perpendicular configuration $\left(\mu_{0} H_{c}=0.33 \mathrm{~T}\right.$ at $2 \mathrm{~K}$ ) is much larger than for the parallel configuration $\left(\mu_{0} H_{c}=0.14 \mathrm{~T}\right.$ at $\left.2 \mathrm{~K}\right)$. From Nishio et al. 2009 .

Coulomb interactions upon the chemical forces between TM ions. The magnetic properties are closely correlated with the heterogeneity of the $\mathrm{Cr}$ distribution. In particular, the Cr-rich regions give rise to the appearance of high-temperature ferromagnetism. The systematic investigations on $(\mathrm{Zn}, \mathrm{Cr}) \mathrm{Te}$ films grown under various $\mathrm{MBE}$ conditions revealed that the growth temperature plays a crucial role in the phase separation: the crystallographic structure and the shape of the Cr-rich regions change with the substrate temperature during the growth. The ability to fabricate in a self-organized fashion hybrid systems consisting of FM nanoclusters embedded in the semiconductor matrix, as realized in (Zn,Cr)Te and other DMSs, opens doors for studies of various functionalities of these novel nanocomposites.

\section{PROSPECTS OF SPINODAL NANOTECHNOLOGY}

It is believed that the application of embedded metallic NCs will revolutionize the performance of various commercial devices, such as flash memories. Similarly colloidal or embedded semiconducting NCs, i.e., semiconductor quantum dots and nanowires, are extensively studied as perspective media for lightning, low current semiconductor lasers, solar cells, single-photon emitters and detectors, quantum processors and memories.

In view of the results presented, the incorporation of TM impurities into semiconductors opens the door to fabrication of dense arrays of TM-rich NCs coherently embedded into a semiconductor matrix. Depending on growth and processing conditions as well as on the combination of host semiconductor, TM impurity, and codoping the self-assembled NCs can be metallic or semiconducting, form nanodots or nanocolumns, exhibit FM, ferrimagnetic, or AF spin ordering persisting usually to above RT. Thus, spinodal nanotechnology not only allows one to explore the feasibility of device fabrication with a new and versatile bottom-up method but also considerably enlarges the spectrum of possible functionalities offered by nanoscale heterogeneous systems (Dietl, 2008c; Katayama-Yoshida et al., 2007a).

As an example, Fig.79 presents results of Monte Carlo simulations in which the Cr flux was altered during the 2D growth of CrTe nanocolumns (the konbu phase) embedded in ZnTe (Fukushima et al., 2007 ). This example suggests that dense arrays of various nanodevices can be fabricated by selecting appropriate growth protocols. For instance, by reducing the flux for time corresponding to the growth of a few monolayers, the formation of magnetic tunnel junctions is predicted for nanocolumns of a FM metal (Fukushima et al., 2007). Such junctions can serve for low-power high-density magnetic storage, including spin-torque magnetic random access memories and, if sufficiently high TMR is found, for the field programmable logic, i.e., TMR-based connecting/disconnecting switches (Reiss and Meyners, 2006), and even all-magnetic logic, characterized by lowpower consumption and radiation hardness. Furthermore, nanocolumns might form racetracks for domainwall based 3D memories (Thomas et al. 2007).

Another kind of possible spintronic applications makes use of the coupling between FM leads and carriers in a semiconductor matrix. In particular, in the proposal of a scalable processor (Dery et al., 2007), the magnetizationdependent interaction between neighbor FM contacts is mediated by spin currents injected from the FM contact to the semiconductor. Furthermore, in the pioneering design of quantum processers involving the couplings between single spins on quantum dots, nanomagnets serve to introduce differences in spin resonance frequencies of particular dots, which makes it possible to address individual spins (Loss and DiVincenzo, 1998). Actually, top-down methods were already employed to pattern nanostructures with small magnets whose stray 

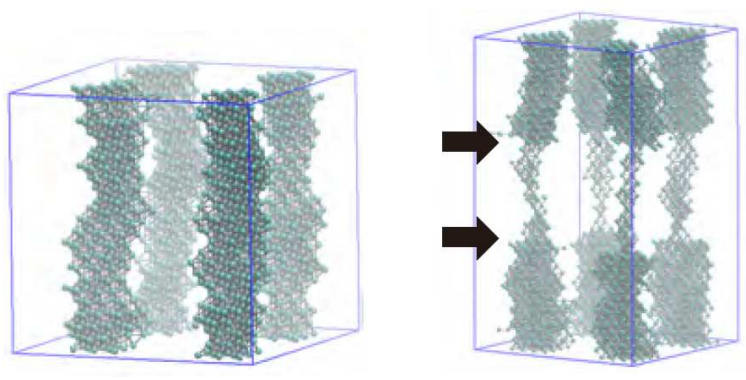

FIG. 79 (Color online) Demonstration of the nanocolumn shape control by Monte Carlo simulations of (Zn,Cr)Te epitaxy (the konbu phase). Positions of $\mathrm{Cr}$ cations are shown. (a) Nanoscale seeding by zinc-blende CrTe. (b) During the deposition (between two arrows), the $\mathrm{Cr}$ concentration is reduced to control the shape of the metallic and FM CrTe nanocolumns embedded in ZnTe. Adapted from Fukushima et al. 2007 .

fields controlled spin currents (Wróbel et al., 2004) and spin resonance frequencies (Pioro-Ladrière et al. 2008) at the nanoscale.

As discussed in Secs. III, VI, VII, and VIII, a number of decomposed alloys exhibits spin-related magnetotransport and magnetooptical phenomena typically persisting up to $T_{\mathrm{C}}$ of the relevant NCs. In particular, AHE was observed in (Ge,Mn) (Jamet et al. 2006) and (Zn,Cr) Te (Kuroda et al., 2007). A sizable magnitude of AHE together with heterogeneity-induced mixing between diagonal and off-diagonal conductivity tensor components resulted in a large positive MR of (Ge,Mn) (Jamet et al. 2006). A related effect was also observed in granular films with $\mathrm{MnSb}$ (Akinaga et al. $2000 \mathrm{~b})$ and $\mathrm{MnAs}$ (Kudrin et al. 2014, Yokoyama et al. 2006) NCs. Furthermore, TMR was found in a device containing MnAs and GaAs:MnAs electrodes (Hai et al. 2008,2006$)$. All these findings could possibly be exploited in magnetic-field sensors with characteristics (e.g., impedance) that could be tailored over a wide range.

At the same time, as discussed in Secs.III, VI, VII and VIII. MCD was reported for decomposed (Ga,Mn)As, $(\mathrm{Ge}, \mathrm{Mn}),(\mathrm{Ge}, \mathrm{Fe})$, and $(\mathrm{Zn}, \mathrm{Cr}) \mathrm{Te}$ as well as for other systems such as (Ga,Mn)P (Monette et al., 2010$)$. A combination of strong and spectrally broad MCD specific to FM metals and weak losses characterizing the semiconductor hosts suggests possible applications of decomposed semiconductor alloys as optical isolators Amemiya et al. 2006) as well as 3D tunable photonic crystals and spatial light modulators for advanced photonic applications (Park et al. 2002a).

Metallic nanocolumns and nanodots could also serve as high quality nanocontacts in nanoelectronics and optoelectronics as well as metallic elements in nanoplasmonics. Dense arrays of nanocolumns are also attractive for thermoelectric applications (power generators and coolers) as a specific form of density of states in 1D systems is expected to result in a significant enhancement of the
Seebeck and Peltier effects (Shinya et al., 2014: Vu et al., 2014a, 2015, 2011, 2014b). Furthermore, the growth process presented in Fig.779 can be adapted to fabricate devices for all-metal nanoelectronics based, for instance, on single-electron transistors. Actually, the Coulomb blockade was demonstrated for a single MnAs NC, tunnelcoupled to MnAs electrodes patterned lithographically (Hai et al. 2010). A long spin relaxation time, of the order of $10 \mu \mathrm{s}$, was evaluated from these data.

If, in contrast, nanocolumns were semiconducting, appropriate band-gap engineering could improve efficiency of photovoltaic solar cells by leading to spatial separation of photoelectrons and photoholes in such allsemiconductor superstructures (Oshitani et al., 2011; Tani et al., 2010, 2011, 2012a b c). As one more example of possible functionalities worth mentioning is the case of catalysts for automotive-emissions control. Here, by using decomposed alloy with spatially separated NCs containing the relevant metal, e.g., Pt, the destructive process of metal agglomeration could be much reduced (Hamada et al., 2011, Katz et al., 2011, Kizaki and Katayama-Yoshida, 2013, Kizaki et al., 2008).

It worth adding that crystallographic phase separation has been extensively studied in III-V semiconductors doped with rare earths (Buehl et al. 2010 , Clinger et al., 2012, Kawasaki et al. 2013). In these systems, self-assembled rock-salt ErAs, TbAs, or ErSb nanocrystals, in the form of either nanodots or nanocolumns embedded in zinc-blende GaAs, (In,Ga)As, or GaSb hosts, respectively were found depending on epitaxy conditions. The suitability of these nanocomposites for thermoelectric, plasmonic, and $\mathrm{THz}$ applications has already been addressed experimentally (Clinger et al., 2012, Lu et al. 2014 Salas et al. 2015).

\section{SUMMARY AND OUTLOOK}

We reviewed the recent progress in the understanding of high-temperature ferromagnetism in a range of magnetically doped semiconductors, including primarily $(\mathrm{Ga}, \mathrm{Mn}) \mathrm{As},(\mathrm{Ga}, \mathrm{Mn}) \mathrm{N},(\mathrm{Ga}, \mathrm{Fe}) \mathrm{N},(\mathrm{Ge}, \mathrm{Mn}),(\mathrm{Ge}, \mathrm{Fe})$, and $(\mathrm{Zn}, \mathrm{Cr}) \mathrm{Te}$. As we have emphasized, the abundance of contradicting views on the mechanisms accounting for surprisingly large magnitudes of Curie temperature $T_{\mathrm{C}}$ resulted from intertwined theoretical and experimental challenges requiring the application of cutting-edge computational and materials nanocharacterization methods that have mostly become available only recently. In this way, semiconductors doped by transition metals (TMs) have emerged as outstanding systems to test our understanding of unanticipated relationships among (i) growth conditions, codoping, and processing; (ii) alloy nanostructure; and (iii) pertinent macroscopic properties.

The key ingredient of the materials families described in this review is spinodal nanodecomposition leading to the formation of TM-rich nanocrystals (NCs) either commensurate with the TM-depleted host (chemical phase 
separation) or precipitating in another crystallographic and/or chemical form (crystallographic phase separation). Whether magnetic ions are distributed randomly or form aggregates is determined by the competition between attractive forces among TM cations (revealed by $a b$ initio computations) and kinetic barriers for TM diffusion at the growth surface or in the bulk. Accordingly, the alloy decomposition is more efficient at high growth temperatures and slow growth rates, and depends also on the Fermi level position (codoping) that changes TMs valence and their diffusion coefficients. Remarkably, according to both theoretical simulations and experimental results the TM-rich regions (condensed magnetic semiconductors - CMSs) assume the form of either nanodots (the dairiseki phase) or nanocolumns (the konbu phase). Furthermore, the nanodots can be distributed randomly or accumulate in a plane adjacent to the interface or surface. Similarly, nanocolumns can extend along the growth direction or assume another spatial orientation.

A rich spectrum of forms assumed by spinodal nanodecomposition, such as chemical phase separation or aggregation of precipitates in one plane, have elucidated the reason why uncovering the presence of a nonrandom distribution of magnetic ions was so challenging in DMS research and, in particular, why the application of standard in-house structure characterization techniques (e.g., $\mathrm{x}$-ray diffraction) was often misleading. The comprehensive element-specific nanocharacterization investigations described have demonstrated the existence of a tight correlation between the presence of TM-rich NCs and high$T_{\mathrm{C}}$ ferromagnetism. Within this scenario the puzzling $T_{\mathrm{C}}$ independence of the average TM concentration $x$ has been explained. However, it has been found that the reverse is not true, i.e., variations of $T_{\mathrm{C}}$ with $x$ do not prove a uniform distribution of TM ions. Furthermore, according to the evidence, spontaneous magnetization of decomposed systems is usually smaller than expected from the TM concentration, as typically a part of TM ions is distributed randomly giving rise to a paramagnetic, and not FM or superparamagnetic response. It also happens that NCs formed by spinodal decomposition are weak ferromagnets or even antiferromagnets [see, e.g., the data for $(\mathrm{Ga}, \mathrm{Fe}) \mathrm{N}$ in Sec.V] or that coupling between them results rather in a superferromagnetic behavior than in a superparamagnetic phase (Sawicki et al. 2013).

It was often argued that the presence of the magnetic circular dichroism (MCD) points to a random distribution of TM ions over cation sites (Ando, 2006). Actually, because of boundary conditions for electromagnetic waves, the dielectric function of heterogeneous media contains contributions from all constituents. Hence, TM-rich NCs not only give a specific contribution to the magnetic response but, in general, can also affect, in a magnetization-dependent fashion, magnetooptical properties, as observed for (Ga,Mn)As, (Ge,Mn), (Ge,Fe), and $(\mathrm{Zn}, \mathrm{Cr}) \mathrm{Te}$ (Secs. III, [VI, $\mathrm{VII}$, and VIII, respectively). In particular, metallic NCs rather than enhancing MCD only at host critical points of the Brillouin zone produce a large MCD signal over a wide spectral range. However, no enhancement of MCD has been reported for $(\mathrm{Ga}, \mathrm{Mn}) \mathrm{N}$ and $(\mathrm{Ga}, \mathrm{Fe}) \mathrm{N}$ (Secs. IV and $\mathrm{V}$, respectively). Presumably, in the case of $(\mathrm{Ga}, \overline{\mathrm{Mn}}) \mathrm{N}$, Mn-rich NCs are wide band-gap insulators, whereas in $(\mathrm{Ga}, \mathrm{Fe}) \mathrm{N}$ the NCs aggregate in a narrow plane parallel to the film surface, which reduces their coupling to light.

A similar question arises to what extent magnetotransport studies could tell whether the TM distribution is uniform or one deals rather with spinodal nanodecomposition. A weak negative contribution to the Hall signal, resembling the anomalous Hall effect (AHE), can originate from stray fields generated by magnetic NCs. More often, spin-dependent coupling between host charge current and magnetic NCs results in a sizable AHE, as observed in (Ge,Mn) and ( $\mathrm{Zn}, \mathrm{Cr}) \mathrm{Te}$, and mentioned in Secs.VI andVIII Particularly challenging is the question of magnetoresistance (MR). Here positive MR, specific to semiconductor-metallic nanocomposites (Solin et al. 2000), may appear, particularly if the magnitude of AHE is significant, the effect being discussed for $(\mathrm{Ge}, \mathrm{Mn})$ in Sec.VI. Moreover, as in other nonmagnetic and magnetic semiconductors, one expects a range of MRs associated with quantum localization phenomena (Dietl, 2008b). In particular, strong spin disorder scattering near $T_{\mathrm{C}}$ accounts for colossal negative MR. However, a weak negative MR away from $T_{\mathrm{C}}$, according to straightforward and parameter-free theoretical modeling, originates rather from the influence of magnetic flux upon interference of scattered carrier de Broglie waves (weak localization MR) than from spin-disorder scattering. This is in contrast with magnetically doped metals in which effects of spin-disorder scattering dominate, as competing scattering mechanisms and associated localization effects are typically weak.

Despite the recent progress, there is a number of challenging and open questions ahead. As an example we note that an important theoretical issue is the determination of kinetic barriers for TM diffusion at the growth surface and in the bulk, crucial parameters to simulate quantitatively the NC assembly during epitaxy, codoping, or postgrowth annealing. Furthermore, since CMSs assume a form imposed by the matrix, their chemical composition and associated properties are by no means obvious. In particular, it is hardly known whether open $d$ shells remain localized in CMSs (as in the parent DMS) or rather a Mott-Hubbard transition occurs, leading to itinerant magnetism specific to certain end compounds such as MnAs and CrTe. It was actually suggested that Mn-rich zinc-blende (zb) NCs in decomposed (Ga,Mn)As contain only about $20 \%$ of $\mathrm{Mn}$ and retain the properties of $(\mathrm{Ga}, \mathrm{Mn}) \mathrm{As}$ in which spins are localized (LawniczakJablonska et al., 2011). A related question concerns the crystalline magnetic anisotropy of particular combinations of CMSs and hosts. This anisotropy, together with shape anisotropy and dipolar or exchange coupling between NCs, accounts for macroscopic magnetic properties, including the apparent magnitude of $T_{\mathrm{C}}$ and the 
character of magnetic hystereses. A theoretical and experimental evaluation of this anisotropy awaits for future studies.

Furthermore, FM-like features brought about by uncompensated spins at the surface of antiferromagnetic (AF) NCs, as discussed theoretically (Dietl et al. 2007), have not yet been put into evidence experimentally in the materials considered here. We also note that the FM proximity effect or the exchange bias in the case of $\mathrm{AF}$ CMSs can lead to spin polarization of a semiconductor surrounding a given NC. Such induced polarization will persist up to the spin ordering temperature of the CMS and, according to the RKKY theory, will extend over a distance of the order of the inverse Fermi vector in the presence of band carriers and perhaps over two or three bond lengths in their absence. This effect was examined in $\mathrm{Fe} /(\mathrm{Ga}, \mathrm{Mn})$ As heterostructures (Maccherozzi et al. 2008 ) but not yet in decomposed alloys, in which it can lead to an erroneous conclusion that the host is intrinsically FM up to high temperatures.

While theoretical and experimental results described in this review are concerned with a limited set of compounds, there is a common ground to expect that the developed methodology, the observed phenomena, and the conclusions drawn from theoretical and experimental results apply to a much broader class of magnetically doped materials and also to many other alloys. We are quite certain that with further development and with wide-spread use of powerful nanocharacterization tools, the family of decomposed alloys will steadily grow. Future work will also show the role of structural defects, residual impurities (e.g. H) or contaminates (e.g. Fe-rich nanoparticles) in the appearance of high- $T_{\mathrm{C}}$ ferromagnetism in semiconductors and oxides.

It is clear that the understanding of the origin of high$T_{\mathrm{C}}$ ferromagnetism and detailed knowledge on the TM distribution are preconditions for a meaningful design of devices that could exploit the properties of magnetically doped semiconductors and insulators. As discussed in Sec.IX, the demonstrated and predicted functionalities of decomposed magnetic alloys might be of interest not only for spintronics but also for electronics, photonics, plasmonics, photovoltaics, thermoelectrics, and catalysis. In general terms, the future studies of these systems will contribute to address the timely question of to what extent and when bottom-up technologies will start to be competitive with the top-down approaches dominating today.

\section{List of abbreviations}

$\begin{array}{ll}1 \mathrm{D}, 2 \mathrm{D}, 3 \mathrm{D} \text { One-, two-, three-dimensional } \\ \text { AF } & \text { Antiferromagnetic } \\ \text { AP } & \text { Atom probe } \\ \text { AFM } & \text { Atomic force microscopy } \\ \text { AHE } & \text { Anomalous Hall effect } \\ \text { CEMS } & \text { Conversion electron Mössbauer spectroscopy }\end{array}$

\begin{tabular}{|c|c|}
\hline CMS & Condensed magnetic semiconductor \\
\hline CPA & Coherent potential approximation \\
\hline DFT & Density functional theory \\
\hline DMS & Dilute magnetic semiconductors \\
\hline DFS & Dilute ferromagnetic semiconductor \\
\hline EDS & $\begin{array}{l}\text { Energy dispersive x-ray spectroscopy } \\
\text { (the same as EDX) }\end{array}$ \\
\hline EELS & Electron energy loss spectroscopy \\
\hline EPR & Electron paramagnetic resonance \\
\hline $\mathrm{EF}$ & Energy-filtered \\
\hline EXAFS & Extended x-ray Absorption Fine Structure \\
\hline $\mathrm{FC}$ & Field cooled \\
\hline fcc & face-centered cubic \\
\hline FM & Ferromagnetic \\
\hline FMR & Ferromagnetic resonance \\
\hline FR & Ferrimagnetic \\
\hline GGA & Generalized gradient approximations \\
\hline GISAXS & Grazing incidence small angle $\mathrm{x}$-ray scattering \\
\hline GMR & Giant magnetoresistance \\
\hline $\mathrm{HR}$ & High resolution \\
\hline LDA & Local density approximation \\
\hline LSDA & Local spin density approximation \\
\hline LT & Low temperature \\
\hline MBE & Molecular beam epitaxy \\
\hline MOVPE & $\begin{array}{l}\text { Metal organic vapor phase deposition } \\
\text { (the same as MOCVD) }\end{array}$ \\
\hline MCD & Magnetic circular dichroism \\
\hline MR & Magnetoresistance \\
\hline $\mathrm{NC}$ & Nanocrystal \\
\hline RHEED & Reflection high-energy electron diffraction \\
\hline RPA & Random phase approximation \\
\hline RKKY & Ruderman-Kittel-Kasuya-Yosida \\
\hline SEM & Scanning electron microscopy \\
\hline SIMS & Secondary ions mass spectroscopy \\
\hline SQUID & Superconducting quantum interference device \\
\hline STM & Scanning tunneling microscopy \\
\hline SXRD & Synchrotron x-ray diffraction \\
\hline TEM & Transmission electron microscopy \\
\hline $\mathrm{TM}$ & Transition metal \\
\hline TMR & Tunneling magnetoresistance \\
\hline wZ & wurtzite \\
\hline XANES & X-ray absorption near edge structure \\
\hline XAS & X-ray absorption spectroscopy \\
\hline XES & X-ray emission spectroscopy \\
\hline XMCD & X-ray magnetic circular dichroism \\
\hline XPEEM & X-ray photoemission electron microscopy \\
\hline $\mathrm{XRD}$ & $\mathrm{X}$-ray diffraction \\
\hline $\mathrm{ZFC}$ & Zero-field cooled \\
\hline $\mathrm{zb}$ & zinc-blende \\
\hline
\end{tabular}

\section{Acknowledgments}

The work of T.D. and A.B. was supported by the European Research Council through the FunDMS Advanced Grant (No.227690) within the "Ideas" 7th Framework Programme of the EC, by Wroclaw Research Centre EIT+ within the project "The Application of Nanotechnology in Advanced Materials" - NanoMat (P2IG.01.01.02-02-002/08) co-financed by the European Regional Development Fund (operational Programme Innovative Economy, 1.1.2), and also (T.D.) by National Center of Science in Poland 
(Decision No. 2011/02/A/ST3/00125). A. B. was supported by the Austrian Fonds zur Förderung der wissenschaftlichen Forschung - FWF (P18942, P20065, P22471, P22477, and P26830) and by the NATO Science for Peace Programme (Project 984735). K. S. acknowledges Ministry of Education, Culture, Sports, Science and Technology (MEXT) KAKENHI (Grant numbers, 22740256, 22104012 and 26286074), the GCOE program "Core Research and Engineering of Advanced Materials-Interdisciplinary Education Center for Materials Science" by MEXT. K.S. also acknowledges the financial support from Strategic Japanese-German Cooperative Program "Computational design and evaluation of spintronics materials" and PREST from Japan Science and Technology Agency (JST). M. J. and A. Ba. was supported by the French Agence Nationale pour la Recherche (ANR project GeMO) and the Nanoscience Foundation of Grenoble (RTRA project IMAGE). M. T. and P. N. H. acknowledge the financial support from the Grant-in-Aid for Specially Promoted Research (No. 23000010) and Grant-in-Aid for Young Scientists (A) (No. 24686040) by JSPS, the Project for Developing Innovation Systems of MEXT, the FIRST program, the Global COE program (C04), and the Advanced Technology Institute (ATI) foundation. The study of S. K. was partially supported by Grant-in-Aids for Scientific Research from Ministry of Education, Science, Sports and Culture of the Japanese Government. H. K.-Y. acknowledges the financial support provided by the Japan Society for the Promotion of Science (JSPS) Core-to-Core Program "Computational Nano-Materials Design on Green Energy", and Advanced Low Carbon Technology Research and Development Program (ALCA) of Japan Science and Technology Agency (JST) "Spinodal Nanotechnology for Super-High Efficiency Energy Conversion". H. K.-Y. and M. T. also acknowledge the stimulated discussion in the meeting of the Cooperative Research Project of the Research Institute of Electrical Communication, Tohoku University. H. K.Y. thanks the Future Research Initiative Group Support Project on Computational Nano-Materials Design: New Strategic Materials, Osaka University.

\section{References}

Abe, E., F. Matsukura, H. Yasuda, Y. Ohno, and H. Ohno, 2000, "Molecular beam epitaxy of III-V diluted magnetic semiconductor (Ga,Mn)Sb," Physica E 7, 981

Abraham, D. W., M. M. Frank, and S. Guha, 2005, "Absence of magnetism in hafnium oxide films," Appl. Phys. Lett. 87, 252502

Aharoni, A., 1996, "Introduction to the theory of ferromagnetism," in The International Series of Monographs on Physics, International Series of Monographs on Physics, Vol. 109, edited by J. Birman, S. F. Edwards, R. H. Friend, C. H. Llewellyn Smith, M. Rees, D. Sherrington, and G. Veneziano (Oxford Science Publications, Oxford)

Ahlers, S., D. Bougeard, N. Sircar, G. Abstreiter, A. Trampert, M. Opel, and R. Gross, 2006, "Magnetic and struc- tural properties of $\mathrm{Ge}_{x} \mathrm{Mn}_{1-x}$ films: Precipitation of intermetallic nanomagnets," Phys. Rev. B 74, 214411

Ahlers, S., P. R. Stone, N. Sircar, E. Arenholz, O. D. Dubon, and D. Bougeard, 2009, "Comparison of the magnetic properties of gemn thin films through mn l-edge x-ray absorption," Appl. Phys. Lett. 95, 151911

Akai, H., 1989, "Fast Korringa-Kohn-Rostoker coherent potential approximation and its application to FCC Ni-Fe systems," J. Phys.: Condens. Matt. 1, 8045

Akai, H., 2002, http://kkr.phys.sci.osaka-u.ac.jp/

Akai, H., and P. H. Dederichs, 1993, "Local moment disorder in ferromagnetic alloys," Phys. Rev. B 47, 8739

Akinaga, H., S. Miyanishi, K. Tanaka, W. Van Roy, and K. Onodera, 2000a, "Magneto-optical properties and the potential application of GaAs with magnetic MnAs nanoclusters," Appl. Phys. Lett. 76, 97

Akinaga, H., M. Mizuguchi, K. Ono, and M. Oshima, 2000b, "Room-temperature thousandfold magnetoresistance change in MnSb granular films: Magnetoresistive switch effect," Appl. Phys. Lett. 76, 357

Akinaga, H., S. Nemeth, J. De Boeck, L. Nistor, H. Bender, G. Borghs, H. Ofuchi, and M. Oshima, 2000c, "Growth and characterization of low-temperature grown GaN with high Fe doping," Appl. Phys. Lett. 77, 4377

Amemiya, T., H. Shimizu, Y. Nakano, Pham Nam Hai, M. Yokoyama, and M. Tanaka, 2006, "Semiconductor waveguide optical isolator based on nonreciprocal loss induced by ferromagnetic MnAs," Appl. Phys. Lett. 89, 021104

Ando, K., 2006, "Seeking room-temperature ferromagnetic semiconductors," Science 312, 1883

Ando, K., A. Chiba, and H. Tanoue, 1998, "Uniaxial magnetic anisotropy of submicron MnAs ferromagnets in GaAs semiconductors," Appl. Phys. Lett. 73, 387

de Andrés, A., A. Espinosa, C. Prieto, M. García-Hernández, R. Ramírez-Jiménez, S. Lambert-Milot, and R. A. Masut, 2011, "MnP films and MnP nanocrystals embedded in GaP epilayers grown on $\mathrm{GaP}(001)$ : Magnetic properties and local bonding structure," J. Appl. Phys. 109, 113910

Arras, E., D. Caliste, T. Deutsch, F. Lancon, and P. Pochet, 2011, "Phase diagram, structure, and magnetic properties of the ge-mn system: A first-principles study," Phys. Rev. B 83, 174103

Arras, E., F. Lancon, and I. Slipukhina, 2012, "Interfacedriven phase separation in multifunctional materials: The case of the ferromagnetic semiconductor gemn," Phys. Rev. B 85, 115204

Barnaś, J., and A. Fert, 1998, "Effects of spin accumulation on single-electron tunneling in a double ferromagnetic microjunction," Europhys. Lett. 44, 85

Baron, T., K. Saminadayar, and N. Magnea, 1998, "Nitrogen doping of Te-based II-VI compounds during growth by molecular beam epitaxy," J. Appl. Phys. 83, 1354

Baur, J., K. Maier, M. Kunzer, U. Kaufmann, and J. Schneider, 1994, "Determination of the GaN/AlN band offset via the (-/0) acceptor level of iron," Appl. Phys. Lett. 65, 2211

Beleggia, M., S. Tandon, Y. Zhu, and M. De Graef, 2004, "On the magnetostatic interactions between nanoparticles of arbitrary shape," J. Magn. Magn. Mater. 278, 270

Beloborodov, I. S., A. V. Lopatin, V. M. Vinokur, and K. B. Efetov, 2007, "Granular electronic systems," Rev. Mod. Phys. 79, 469

Bergqvist, L., O. Eriksson, J. Kudrnovský, V. Drchal, P. Korzhavyi, and I. Turek, 2004, "Magnetic percolation in 
diluted magnetic semiconductors," Phys. Rev. Lett. 93, 137202

Bergqvist, L., K. Sato, H. Katayama-Yoshida, and P. H. Dederichs, 2011, "Computational materials design for high- $T_{c}$ (Ga,Mn)As with Li codoping," Phys. Rev. B 83, 165201

Biegger, E., L. Staheli, M. Fonin, U. Rudiger, and Y. S. Dedkov, 2007, "Intrinsic ferromagnetism versus phase segregation in Mn-doped Ge," J. Appl. Phys. 101, 103912

Bihler, C., H. Huebl, M. S. Brandt, S. T. B. Goennenwein, M. Reinwald, U. Wurstbauer, M. Döppe, D. Weiss, and W. Wegscheider, 2006, "Magnetic anisotropy of $\mathrm{Ga}_{1-x} \mathrm{Mn}_{x}$ As thin films on GaAs(311)A probed by ferromagnetic resonance," Appl. Phys. Lett. 89, 012507

Binns, C., K. N. Trohidou, J. Bansmann, S. H. Baker, J. A. Blackman, J.-P. Bucher, D. Kechrakos, A. Kleibert, S. Louch, K.-H. Meiwes-Broer, G. M. Pastor, A. Perez, and Y. Xie, 2005, "The behaviour of nanostructured magnetic materials produced by depositing gas-phase nanoparticles," J. Phys. D: Appl. Phys. 38, R357

Biquard, X., O. Proux, J. Cibert, D. Ferrand, H. Mariette, R. Giraud, and B. Barbara, 2003, "Local structure and valence state of $\mathrm{Mn}$ in $\mathrm{Ga}_{1-x} \mathrm{Mn}_{x} \mathrm{~N}$ epilayers," J. Supercond. 16, 127

Birowska, M., C. Śliwa, J. A. Majewski, and T. Dietl, 2012, "Origin of bulk uniaxial anisotropy in zinc-blende dilute magnetic semiconductors," Phys. Rev. Lett. 108, 237203

Blinowski, J., P. Kacman, and J. A. Majewski, 1996, "Ferromagnetic superexchange in Cr-based diluted magnetic semiconductors," Phys. Rev. B 53, 9524

Bogusławski, P., and J. Bernholc, 2006, "Properties of wurtzite $w$-MnN and of $w$-MnN inclusions in (Ga,Mn)N," Appl. Phys. Lett. 88, 092502

Bolcal, E., V. Dimitrov, B. Actaş, H. Aslan, and A. Bozkurt, 2012, Acta Phys. Pol. A 121, 257

Bonanni, A., 2007, "Ferromagnetic nitride-based semiconductors doped with transition metals and rare earths," Semicond. Sci. Technol. 22, R41

Bonanni, A., 2011, "(Nano)characterization of semiconductor materials and structures," Semicon. Sci. Technol. 26, 060301

Bonanni, A., M. Kiecana, C. Simbrunner, T. Li, M. Sawicki, M. Wegscheider, M. Quast, H. Przybylińska, A. NavarroQuezada, R. Jakieła, A. Wolos, W. Jantsch, and T. Dietl, 2007, "Paramagnetic GaN:Fe and ferromagnetic (Ga,Fe)N: The relationship between structural, electronic, and magnetic properties," Phys. Rev. B 75, 125210

Bonanni, A., A. Navarro-Quezada, T. Li, M. Wegscheider, Z. Matěj, V. Holý, R. T. Lechner, G. Bauer, M. Rovezzi, F. D'Acapito, M. Kiecana, M. Sawicki, and T. Dietl, 2008, "Controlled aggregation of magnetic ions in a semiconductor: An experimental demonstration," Phys. Rev. Lett. 101, 135502

Bonanni, A., M. Sawicki, T. Devillers, W. Stefanowicz, B. Faina, Tian Li, T. E. Winkler, D. Sztenkiel, A. NavarroQuezada, M. Rovezzi, R. Jakieła, A. Grois, M. Wegscheider, W. Jantsch, J. Suffczyński, F. D'Acapito, A. Meingast, G. Kothleitner, and T. Dietl, 2011, "Experimental probing of exchange interactions between localized spins in the dilute magnetic insulator (Ga,Mn)N," Phys. Rev. B 84, 035206

Bonanni, A., C. Simbrunner, M. Wegscheider, H. Przybylinska, A. Wolos, H. Sitter, and W. Jantsch, 2006, "Doping of GaN with $\mathrm{Fe}$ and $\mathrm{Mg}$ for spintronics applications," phys. stat. sol. (b) 243, 1701
Bougeard, D., S. Ahlers, A. Trampert, N. Sircar, and G. Abstreiter, 2006, "Clustering in a precipitate free GeMn magnetic semiconductor," Phys. Rev. Lett. 97, 237202

Bougeard, D., N. Sircar, S. Ahlers, V. Lang, G. Abstreiter, A. Trampert, J.-M. LeBeau, S. Stemmer, D. W. Saxey, and A. Cerezo, 2009, "Ge $\mathrm{Ge}_{1-x} \mathrm{Mn}_{x}$ clusters: Central structural and magnetic building blocks of nanoscale wire-like selfassembly in a magnetic semiconductor," Nano Lett. 9, 3743

Bouzerar, G., and T. Ziman, 2006, "Model for vacancyinduced $d^{0}$ ferromagnetism in oxide compounds," Phys. Rev. Lett. 96, 207602

Bouzerar, G., T. Ziman, and J. Kudrnovský, 2005, "Calculating the Curie temperature reliably in diluted III-V ferromagnetic semiconductors," Europhys. Lett. 69, 812

Buehl, T.E., J.M. LeBeau, S. Stemmer, M.A. Scarpulla, C.J. Palmstrøm, and A. C. Gossard, 2010, "Growth of embedded ErAs nanorods on (411)A and (411)B GaAs by molecular beam epitaxy," J. Cryst. Growth 312, 2089

Cahn, J. W., 1962, "On spinodal decomposition in cubic crystals," Acta Metall. 10 (3), 179, ISSN 0001-6160

Cahn, J. W., and J. E. Hilliard, 1958, "Free energy of a nonuniform system. i. interfacial free energy," J. Chem. Phys. 28, 258

Ceperly, D. M., and B. J. Alder, 1980, "Ground state of the electron gas by a stochastic method," Phys. Rev. Lett. 45, 566

Chan, J. A., Jefferson Z. Liu, Hannes Raebiger, Stephan Lany, and Alex Zunger, 2008, "Relative stability, electronic structure, and magnetism of $\mathrm{MnN}$ and (Ga,Mn)N alloys," Phys. Rev. B 78, 184109

Chason, E., J. Y. Tsao, K. M. Horn, and S. T. Picraux, 1989, "Dynamics of growth roughening and smoothening on Ge (001)," J. Vac. Sci. Technol. B 7, 332

Chen, Chenjia, Ming Cai, Xuezhong Wang, Shifa Xu, Ming Zhang, Xiaomin Ding, and Yunxi Sun, 2000, "Ferromagnetic properties and structures of the Mn-implanted GaAs semiconductor," J. Appl. Phys. 87, 5636

Chiba, D., Y. Nishitani, F. Matsukura, and H. Ohno, 2007, "Properties of $\mathrm{Ga}_{1-x} \mathrm{Mn}_{x}$ As with high $\mathrm{Mn}$ composition $(x>0.1), "$ Appl. Phys. Lett. 90, 122503

Chinchore, A., K. Wang, W. Lin, J. Pak, and A. R. Smith, 2008, "Atomic layer structure of manganese atoms on wurtzite gallium nitride (0001)," Appl. Phys. Lett. 93, 181908

Cho, S., S. Choi, S. C. Hong, Y. Kim, J. B. Ketterson, B. J. Kim, Y. C. Kim, and J. H. Jung, 2002, "Ferromagnetism in Mn-doped Ge," Phys. Rev. B 66, 033303

Cho, Y. J., C. H. Kim, H. S. Kim, W. S. Lee, S.-H. Park, J. Park, S. Y. Bae, B. Kim, H. Lee, and J.-Y. Kim, 2008a, "Ferromagnetic $\mathrm{Ge}_{1-x} \mathrm{M}_{x}(\mathrm{M}=\mathrm{Mn}, \mathrm{Fe}$, and Co) nanowires," Chem. Mater. 20, 4694

Cho, Y. J., C. H. Kim, H. S. Kim, W. S. Lee, S. H. Park, J. Park, S. Y. Bae, B. Kim, H. Lee, and J. Y. Kim, 2008b, "Ferromagnetic $\mathrm{Ge}_{1-x} \mathrm{M}_{x}(\mathrm{M}=\mathrm{Mn}, \mathrm{Fe}$, and Co) nanowires," Chem. Mater. 20, 4694

Choi, E. A., J. Kang, and K. J. Chang, 2006, "Energetics of cubic and hexagonal phases in Mn-doped GaN: Firstprinciples pseudopotential calculations," Phys. Rev. B 74, 245218

Choi, S., S. C. Hong, S. Cho, Y. Kim, J. B. Ketterson, C.-U. Jung, K. Rhie, B.-J. Kim, and Y. C. Kim, 2003, "Ferromagnetic properties in $\mathrm{Cr}, \mathrm{Fe}-$ doped Ge single crystals," J. Appl. Phys. 93, 7670

Clinger, L.E., G. Pernot, T.E. Buehl, P.G. Burke, A.C. 
Gossard, C.J. Palmstrøm, A. Shakouri, and J.M.O. Zide, 2012, "Thermoelectric properties of epitaxial TbAs:InGaAs nanocomposites," J. Appl. Phys. 111, 094312

Coey, J. M. D., P Stamenov, R. D. Gunning, M. Venkatesan, and K. Paul, 2010, "Ferromagnetism in defect-ridden oxides and related materials," New J. Phys. 12, 053025

Continenza, A., G. Profeta, and S. Picozzi, 2006a, "Transition metal doping and clustering in Ge," Appl. Phys. Lett. 89, 202510

Continenza, A., G. Profeta, and S. Picozzi, 2006b, "Transition metal impurities in Ge: Chemical trends and codoping studied by electronic structure calculations," Phys. Rev. B 73, 035212

Continenza, A., G. Profeta, and S. Picozzi, 2007, "Transition metal doping in Ge," J. Magn. Magn. Mat. 310, 2147

Cui, X. Y., B. Delley, A. J. Freeman, and C. Stampfl, 2006, "Magnetic metastability in tetrahedrally bonded magnetic III-nitride semiconductors," Phys. Rev. Lett. 97, 016402

Cui, X. Y., B. Delley, A. J. Freeman, and C. Stampfl, 2007, "Neutral and charged embedded clusters of $\mathrm{Mn}$ in doped GaN from first principles," Phys. Rev. B 76, 045201

Cui, X. Y., J. E. Medvedeva, B. Delley, A. J. Freeman, N. Newman, and C. Stampfl, 2005, "Role of embedded clustering in dilute magnetic semiconductors: Cr doped GaN," Phys. Rev. Lett. 95, 256404

Cui, Y., and L. Li, 2002, "Suppression of phase segregation during molecular-beam epitaxial growth of GaMnN using nitrogen-hydrogen plasma," Appl. Phys. Lett. 80, 4139

Da Silva, Juarez L. F., Gustavo M. Dalpian, and Su-Huai Wei, 2008, "Carrier-induced enhancement and suppression of ferromagnetism in $\mathrm{Zn}_{1-x} \mathrm{Cr}_{x}$ Te and $\mathrm{Ga}_{1-x} \mathrm{Cr}_{x}$ As: origin of the spinodal decomposition," New J. Phys. 10, 113007

Dalmas de Réotier, P., E. Prestat, P. Bayle-Guillemaud, M. Boukhari, A. Barski, A. Marty, M. Jamet, A. Suter, T. Prokscha, Z. Salman, E. Morenzoni, and A. Yaouanc, 2015, "Evidence for a core-shell nanostructure in Ge:Mn," Phys. Rev. B 91, 245408

Dalpian, G. M., Juarez L. F. Da Silva, and Su-Huai Wei, 2009, "Ferrimagnetic Fe-doped GaN: An unusual magnetic phase in dilute magnetic semiconductors," Phys. Rev. B 79, 241201(R)

Darques, M., A. Encinas, L. Vila, and L. Piraux, 2004, "Tailoring of the c-axis orientation and magnetic anisotropy in electrodeposited Co nanowires," J. Phys.: Condens. Matter 16, S2279

Das, G. P., B. K. Rao, and P. Jena, 2003, "Ferromagnetism in Mn-doped GaN: From clusters to crystals," Phys. Rev. B 68, 035207

De Boeck, J., R. Oesterholt, A. Van Esch, H. Bender, C. Bruynseraede, C. Van Hoof, and G. Borghs, 1996, "Nanometer-scale magnetic MnAs particles in GaAs grown by molecular beam epitaxy," App. Phys. Lett. 68, 2744

Demchenko, I. N., K. Lawniczak-Jablonska, T. Story, V. Osinniy, R. Jakiela, J. Z. Domagala, J. Sadowski, M. Klepka, A. Wolska, and M. Chernyshova, 2007, "Modification of the local atomic structure around $\mathrm{Mn}$ atoms in $(\mathrm{Ga}, \mathrm{Mn})$ As layers by high temperature annealing," J. Phys.: Condens. Matter 19, 496205

Dery, H., P. Dalal, Ł. Cywiński, and L. J. Sham, 2007, "Spinbased logic in semiconductors for reconfigurable large-scale circuits," Nature 447, 573

Devillers, T., 2008, Study of the physical properties of ferromagnetic $G e_{1-x} M n_{x}$ phases for spintronics (in French) (Ph.D. thesis, University of Grenoble-France)
Devillers, T., M. Jamet, A. Barski, V. Poydenot, P. BayleGuillemaud, E. Bellet-Amalric, S. Cherifi, and J. Cibert, 2007a, "Structure and magnetism of self-organized $\mathrm{Ge}_{1-x} \mathrm{Mn}_{x}$ nanocolumns on $\mathrm{Ge}(001)$," Phys. Rev. B 76, 205306

Devillers, T., M. Jamet, A. Barski, V. Poydenot, R. Dujardin, P. Bayle Guillemaud, J. Rothman, E. Bellet Amalric, J. Cibert, R. Mattana, and S. Tatarenko, 2006, "Ferromagnetism of self-organized $\mathrm{Ge}_{1-x} \mathrm{Mn}_{x}$ nano-pillars," phys. stat. sol. (c) 3, 4123

Devillers, T., M. Jamet, A. Barski, V. Poydenot, R. Dujardin, P. Bayle Guillemaud, J. Rothman, E. Bellet Amalric, J. Cibert, R. Mattana, and S. Tatarenko, 2007b, "Structural and magnetic properties of GeMn layers; high Curie temperature ferromagnetism induced by self organized GeMn nano-columns," phys. stat. sol. (a) 204, 130

Devillers, T., M. Rovezzi, N. Gonzalez Szwacki, S. Dobkowska, W. Stefanowicz, D. Sztenkiel, A. Grois, J. Suffczyński, A. Navarro-Quezada, B. Faina, T. Li, P. Glatzel, F. d'Acapito, R. Jakieła, M. Sawicki, J. A. Majewski, T. Dietl, and A. Bonanni, 2012, "Manipulating $\mathrm{Mn}-\mathrm{Mg}_{k}$ cation complexes to control the charge- and spin-state of Mn in GaN," Sci. Rep. 2, 722

Dhar, S., O. Brandt, A. Trampert, L. Däweritz, K. J. Friedland, K. H. Ploog, J. Keller, B. Beschoten, and G. Güntherodt, 2003a, "Origin of high-temperature ferromagnetism in $(\mathrm{Ga}, \mathrm{Mn}) \mathrm{N}$ layers grown on $4 \mathrm{H}-\mathrm{SiC}(0001)$ by reactive molecular-beam epitaxy," Appl. Phys. Lett. 82, 2077

Dhar, S., O. Brandt, A. Trampert, K. J. Friedland, Y. J. Sun, and K. H. Ploog, 2003b, "Observation of spin-glass behavior in homogeneous ( $\mathrm{Ga}, \mathrm{Mn}) \mathrm{N}$ layers grown by reactive molecular-beam epitaxy," Phys. Rev. B 67, 165205

Dietl, T., 1981, "Semimagnetic semiconductors in high magnetic fields," in Physics in High Magnetic Fields, edited by S. Chikazumi and N. Miura (Springer, Berlin) p. 344

Dietl, T., 2002, "Ferromagnetic semiconductors," Semicond. Sci. Technol. 17, 377

Dietl, T., 2003, "Dilute magnetic semiconductors: Functional ferromagnets," Nat. Mater. 2, 646

Dietl, T., 2004, "Spintronics in nitrides," in Materials Research Society Fall Meeting, Boston, USA, Nov. 2004, MRS Proceedings , Vol. 831, edited by C. Wetzel, B. Gil, M. Kuzuhara, and M. Manfra (MRS, Warrendale PA) p. E91

Dietl, T., 2006, "Self-organised growth controlled by charge sates of magnetic impurities," Nat. Mater. 5, 673

Dietl, T., 2008a, "Hole states in wide band-gap diluted magnetic semiconductors and oxides," Phys. Rev. B 77, 085208

Dietl, T., 2008b, "Interplay between carrier localization and magnetism in diluted magnetic and ferromagnetic semiconductors," J. Phys. Soc. Jpn. 77, 031005

Dietl, T., 2008c, "Origin and control of ferromagnetism in dilute magnetic semiconductors and oxides (invited)," J. Appl. Phys. 103, 07D111

Dietl, T., T. Andrearczyk, A. Lipińska, M. Kiecana, Maureen Tay, and Yihong $\mathrm{Wu}, 2007$, "Origin of ferromagnetism in $\mathrm{Zn}_{1-x} \mathrm{Co}_{x} \mathrm{O}$ from magnetization and spin-dependent magnetoresistance measurements," Phys. Rev. B 76, 155312

Dietl, T., and H. Ohno, 2003, "Ferromagnetic III-V and II-VI semiconductors," MRS Bull. 28, 714

Dietl, T., and H. Ohno, 2014, "Dilute ferromagnetic semiconductors: Physics and spintronic structures," Rev. Mod. Phys. 86, 187-251 
Dietl, T., H. Ohno, and F. Matsukura, 2001, "Hole-mediated ferromagnetism in tetrahedrally coordinated semiconductors," Phys. Rev. B 63, 195205

Dietl, T., H. Ohno, F. Matsukura, J. Cibert, and D. Ferrand, 2000, "Zener model description of ferromagnetism in zincblende magnetic semiconductors," Science 287, 1019

Dijkstra, J., H. H. Weitering, C. F. van Bruggen, C. Haas, and R. A. de Groot, 1989, "Band-structure calculations, and magnetic and transport properties of ferromagnetic chromium tellurides (CrTe, $\mathrm{Cr}_{3} \mathrm{Te}_{4}, \mathrm{Cr}_{2} \mathrm{Te}_{3}$ )," J. Phys.: Condens. Matter 1, 9141

Dimitrov, D. A., and G. M. Wysin, 1996, "Magnetic properties of superparamagnetic particles by a Monte Carlo method," Phys. Rev. B 54, 9237

DiPietro, R. S., H. G. Johnson, S. P. Bennett, T. J. Nummy, L. H. Lewis, and D. Heiman, 2010, "Determining magnetic nanoparticle size distributions from thermomagnetic measurements," Appl. Phys. Lett. 96, 222506

D'Orazio, F., F. Lucari, N. Pinto, L. Morresi, and R. Murri, 2004, "Toward room temperature ferromagnetism of Ge:Mn systems," J. Magn. Magn. Mat. 272, 2006

Dreizler, R., and E. Gross, 1995, in Density Functional Theory (Plenum Press, New York)

Droghetti, A., N. Baadji, and S. Sanvito, 2009, "MgN: A possible material for spintronic applications," Phys. Rev. B 80, 235310

Du, Aijun, Stefano Sanvito, and Sean C. Smith, 2012, "Firstprinciples prediction of metal-free magnetism and intrinsic half-metallicity in graphitic carbon nitride," Phys. Rev. Lett. 108, 197207

Ducastelle, F., and F. Gautier, 1976, "Generalized perturbation theory in disordered transitional alloys: Applications to the calculation of ordering energies," J. Phys. F: Met. Phys. 6, 2039

Dwiliński, R., R. Doradziński, J. Garczyński, L. Sierzputowski, J. M. Baranowski, and M. Kamińska, 1998, "AMMONO method of GaN and AlN production," Diamond Relat. Mater. 7, 1348)

Edmonds, K. W., P. Bogusławski, K. Y. Wang, R. P. Campion, N. R. S. Farley, B. L. Gallagher, C. T. Foxon, M. Sawicki, T. Dietl, M. B. Nardelli, and J. Bernholc, 2004, "Mn interstitial diffusion in (Ga,Mn)As," Phys. Rev. Lett. 92, 037201

Elfimov, I. S., S. Yunoki, and G. A. Sawatzky, 2002, "Possible path to a new class of ferromagnetic and half-metallic ferromagnetic materials," Phys. Rev. Lett. 89, 216403

Ellner, M., 1980, "Kristallstrukturdaten von $\mathrm{Mn}_{2} \mathrm{Ge}$," J. Appl. Cryst. 13, 99

Fert, A., and H. Jaffrès, 2001, "Conditions for efficient spin injection from a ferromagnetic metal into a semiconductor," Phys. Rev. B 64, 184420

Flory, J. P., 1942, "Thermodynamics of high polymer solutions," J. Chem. Phys. 10, 51

Forsyth, J. B., and P. J. Brown, 1990, "The spatial distribution of magnetisation density in $\mathrm{Mn}_{5} \mathrm{Ge}_{3}$," J. Phys.: Condens. Matter 2, 2713

Fukumura, T., and M. Kawasaki, 2013, "Magnetic oxide semiconductors: On the high-temperature ferromagnetism in $\mathrm{TiO}_{2-}$ and ZnO-based compounds," in Functional Metal Oxides (Wiley-VCH Verlag GmbH \& Co. KGaA, Weinheim) pp. 89-131

Fukushima, T., K. Sato, H. Katayama-Yoshida, and P. H. Dederichs, 2004, "Theoretical prediction of Curie temperature in $(\mathrm{Zn}, \mathrm{Cr}) \mathrm{S},(\mathrm{Zn}, \mathrm{Cr}) \mathrm{Se}$ and $(\mathrm{Zn}, \mathrm{Cr}) \mathrm{Te}$ by first prin- ciples calculations," Jpn. J. Appl. Phys. 43, L1416

Fukushima, T., K. Sato, H. Katayama-Yoshida, and P. H. Dederichs, 2006a, "Ab initio study of spinodal decomposition in (Zn,Cr)Te," Phys. Stat. Sol. A 203, 2751

Fukushima, T., K. Sato, H. Katayama-Yoshida, and P. H. Dederichs, 2006b, "Spinodal decomposition under layer by layer growth condition and high Curie temperature quasione-dimensional nano-structure in dilute magnetic semiconductors," Jpn. J. Appl. Phys. 45, L416

Fukushima, T., K. Sato, H. Katayama-Yoshida, and P. H. Dederichs, 2007, "Ab initio design of fabrication process and shape control of self-organized Tera-bit-density nano-magnets in dilute magnetic semiconductors by twodimensional spinodal decomposition," phys. stat. sol. (c) 3, 4139

Furdyna, J. K., and J. Kossut, 1988, Diluted Magnetic Semiconductors, Semiconductors and Semimetals, Vol. 25 (Academic Press, New York)

Gambardella, P., H. Brune, S. S. Dhesi, P. Bencok, S. R. Krishnakumar, S. Gardonio, M. Veronese, C. Grazioli, and C. Carbone, 2005, "Paramagnetic Mn impurities on Ge and GaAs surfaces," Phys. Rev. B 72, 045337

Garcia, J., 1985, "Magnetic susceptibility, magnetization and specific heat of $\mathrm{Mn}_{3.2} \mathrm{Ga}_{0.8} \mathrm{~N}$," J. Magn. Magn. Mater. 51, 365

Giebultowicz, T. M., P. Kłosowski, N. Samarth, H. Luo, J. K. Furdyna, and J. J. Rhyne, 1993, "Neutron-diffraction studies of zinc-blende MnTe epitaxial films and MnTe/ZnTe superlattices: The effect of strain and dilution on a strongly frustrated fcc antiferromagnet," Phys. Rev. B 48, 12817

Giraud, R., S. Kuroda, S. Marcet, E. Bellet-Amalric, X. Biquard, B. Barbara, D. Fruchart, D. Ferrand J. Cibert, and H. Mariette, 2004, "Ferromagnetic (Ga,Mn)N epilayers versus antiferromagnetic $\mathrm{GaMn}_{3} \mathrm{~N}$ clusters," Europhys. Lett. 65, 553

Godlewski, M., and M. Kamińska, 1980, "The chromium impurity photogeneration transitions in $\mathrm{ZnS}, \mathrm{ZnSe}$ and ZnTe," J. Phys. C: Solid State Phys. 13, 6537

Gonis, A., 2000, in Theoretical Materials Science (Materials Research Society, Warrendale PA)

Gonzalez Szwacki, N., J. A. Majewski, and T. Dietl, 2011, "Aggregation and magnetism of $\mathrm{Cr}, \mathrm{Mn}$, and Fe cations in GaN," Phys. Rev. B 83, 184417

Gosk, J., M. Zajac, M. Byszewski, M. Kamińska, J. Szczytko, A. Twardowski B. Strojek, and S. Podsiało, 2003, "Magnetic properties of $(\mathrm{Ga}, \mathrm{Fe}) \mathrm{N}$," J. Supercond. Nov. Magn. 16, 79

Grace, P. J., M. Venkatesan, J. Alaria, J. M. D. Coey, G. Kopnov, and R. Naaman, 2009, "The origin of the magnetism of etched Silicon," Adv. Mater. 21, 71

Graf, T., S. T. B. Goennenwein, and M. S. Brandt, 2003, "Prospects for carrier-mediated ferromagnetism in GaN," phys. stat. sol. (b) 239, 277

Granville, S., B. J. Ruck, F. Budde, H. J. Trodahl, and G. V. M. Williams, 2010, "Nearest-neighbor mn antiferromagnetic exchange in $\mathrm{Ga}_{1-x} \mathrm{Mn}_{x} \mathrm{~N}$," Phys. Rev. B 81, 184425

Grois, A., T. Devillers, Tian Li, and A. Bonanni, 2014, "Planar array of self-assembled $\mathrm{Ga}_{x} \mathrm{Fe}_{4-x} \mathrm{~N}$ nanocrystals in GaN: magnetic anisotropy determined via ferromagnetic resonance," Nanotechnology 25 (39), 395704

Gu, L., S. Wu, H. Liu, R. Singh, N. Newman, and D. Smith, 2005, "Characterization of $\mathrm{Al}(\mathrm{Cr}) \mathrm{N}$ and $\mathrm{Ga}(\mathrm{Cr}) \mathrm{N}$ dilute magnetic semiconductors," J. Magn. Magn. Mater. 290, 
1395

Gupta, S., W. E. Fenwick, A. Melton, T. Zaidi, H. Yua, V. Rengarajan, J. Nause, A. Ougazzaden, and I. T. Ferguson, 2008, "MOVPE growth of transition-metal-doped GaN and $\mathrm{ZnO}$ for spintronic applications," J. Cryst. Growth 310, 5032

Gupta, S., H. Kang, M. Strassburg, A. Asghar, M. Kane, W. E. Fenwick, N. Dietz, and I. T. Ferguson, 2006, "A nucleation study of group III-nitride multifunctional nanostructures," J. Cryst. Growth 287, 596

Hai, Pham Nam, W. Nomura, T. Yatsui, M. Ohtsu, and M. Tanaka, 2012, "Effects of laser irradiation on the selfassembly of MnAs nanoparticles in a GaAs matrix," Appl. Phys. Lett. 101, 193102

Hai, Pham Nam, S. Ohiya, M. Tanaka, S. E. Barnes, and S. Maekawa, 2009, "Electromotive force and huge magnetoresistance in magnetic tunnel junctions," Nature 458, 489

Hai, Pham Nam, S. Ohya, and M. Tanaka, 2010, "Long spinrelaxation time in a single metal nanoparticle," Nature Nanotech. 5, 593

Hai, Pham Nam, Y. Sakata, M. Yokoyama, S. Ohya, and M. Tanaka, 2008, "Spin-valve effect by ballistic transport in ferromagnetic metal (MnAs)/semiconductor (GaAs) hybrid heterostructures," Phys. Rev. B 77, 214435

Hai, Pham Nam, K. Takahashi, M. Yokoyama, S. Ohya, and M. Tanaka, 2007, "Magnetic properties of MnAs nanoclusters embedded in a GaAs semiconductor matrix," J. Magn. Magn. Mater. 310, 1932

Hai, Pham Nam, S. Yada, and M. Tanaka, 2011, "Phase decomposition diagram of magnetic alloy semiconductor," J. Appl. Phys. 109, 073919

Hai, Pham Nam, M. Yokoyama, S. Ohya, and M. Tanaka, 2006, "Tunneling magnetoresistance of MnAs thin film/GaAs/AlAs/GaAs:MnAs nanoclusters and its AlAs barrier thickness dependence," Appl. Phys. Lett. 89, 242106

Hamada, I., A. Uozumi, Y. Morikawa, A. Yanase, and H. Katayama-Yoshida, 2011, "A density functional theory study of self-regenerating catalysts $\mathrm{LaFe}_{1-x} \mathrm{M}_{x} \mathrm{O}_{3-y}(\mathrm{M}=$ Pd, Rh, Pt)," J. Am. Chem. Soc. 133, 18506

Han, Y., M. W. Fay, P. D. Brown, S. V. Novikov, K. W. Edmonds, B. L. Gallagher, R. P. Campion, and C. T. Foxon, 2007, "Microstructural characterization of low-temperature grown GaMnN on $\operatorname{GaAs}\left(\begin{array}{lll}0 & 0 & 1\end{array}\right)$ substrates by plasmaassisted MBE," Semicond. Sci. Technol. 22, 1131

Hara, S., and T. Fukui, 2006, "Hexagonal ferromagnetic MnAs nanocluster formation on GaInAs/InP (111)B layers by metal-organic vapor phase epitaxy," Appl. Phys. Lett. 89, 113111

Hara, S., and A. Kuramata, 2005, "Ferromagnetic nanoclusters hybridized in Mn-incorporated GaInAs layers during metal-organic vapour phase epitaxial growth on InP layers under low growth temperature conditions," Nanotechnology 16, 957

Hayashi, T., M. Tanaka, T. Nishinaga, H. Shimada, H. Tsuchiya, and Y. Otuka, 1997, "(GaMn)As: GaAsbased III-V diluted magnetic semiconductors grown by molecular beam epitaxy," J. Crys. Growth 175-176, Part 2, 1063

Heikman, S., S. Keller, T. Mates, S. P. DenBaars, and U. K. Mishra, 2003, "Growth and characteristics of Fe-doped GaN," J. Crys. Growth 248, 513

Hilbert, S., and W. Nolting, 2004, "Disorder in diluted spin systems," Phys. Rev. B 70, 165203

Hinomura, T., and S. Nasu, 1996, " ${ }^{57}$ Fe Mössbauer study of Fe nitrides," Nouvo Cimento 18, 253

Hohenberg, P., and W. Kohn, 1964, "Inhomogeneous electron gas," Phys. Rev 136, B864

Houben, A., J. Burghaus, and R Dronskowski, 2009, "The ternary nitrides $\mathrm{GaFe}_{3} \mathrm{~N}$ and $\mathrm{AlFe}_{3} \mathrm{~N}$ : Improved synthesis and magnetic properties," Chem. Mater. 21, 4332

Huggins, M. L., 1941, "Solutions of long chain compounds," J. Chem. Phys. 9, 440

Hwang, J. I., Y. Ishida, M. Kobayashi, H. Hirata, K. Takubo, T. Mizokawa, A. Fujimori, J. Okamoto, K. Mamiya, Y. Saito, Y. Muramatsu, H. Ott, A. Tanaka, T. Kondo, and H. Munekata, 2005, "High-energy spectroscopic study of the III-V nitride-based diluted magnetic semiconductor $\mathrm{Ga}_{1-x} \mathrm{Mn}_{x} \mathrm{~N}$," Phys. Rev. B 72, 085216

Hwang, J. I., Y. Osafune, M. Kobayashi, K. Ebata, Y. Ooki, Y. Ishida, A. Fujimori, Y. Takeda, T. Okane, Y. Saitoh, K. Kobayashi, and A. Tanaka, 2007, "Depth profile highenergy spectroscopic study of Mn-doped GaN prepared by thermal diffusion," J. Appl. Phys. 101, 103709

Hynninen, T., H. Raebiger, and J. von Boehm, 2007, "Structural and magnetic properties of $(\mathrm{Ga}, \mathrm{Mn}) \mathrm{N}$ from first principles," Phys. Rev. B 75, 125208

Hynninen, T., H. Raebiger, J. von Boehm, and A. Ayuela, 2006a, "High Curie temperatures in (Ga,Mn)N from Mn clustering," Appl. Phys. Lett. 88, 122501

Hynninen, T., H. Raebiger, and J. von Boehm, 2006b, "A multiscale study of ferromagnetism in clustered (Ga,Mn)N," J. Phys.: Conden. Matter 18 (5), 1561

Ishikawa, K., N. Nishizawa, S. Kuroda, H. Ikeda, K. Takita, M. Mitome, Y. Bando, and T. Dietl, 2009, "Inhomogeneous $\mathrm{Cr}$ distribution and superparamagnetic behavior in magnetic semiconductor (Zn,Cr)Te," Physics of Semiconductors, AIP Conf. Proc. 1199, 419

Ivanovskii, A. L., 2007, "Magnetic effects induced by sp impurities and defects in nonmagnetic sp materials," PhysicsUspekhi 50 (10), 1031

Jack, K. H., 1952, "The iron-nitrogen system: the crystal structures of $\epsilon$-phase iron nitrides," Acta Cryst. 5, 404

Jacobs, H., D. Rechenbach, and U. Zachwieja, 1995, "Structure determination of $\gamma^{\prime}-\mathrm{Fe}_{4} \mathrm{~N}$ and $\epsilon-\mathrm{Fe}_{3} \mathrm{~N}$," J. Alloys Compd. 227, 10

Jaeger, C., C. Bihler, T. Vallaitis, S. T. B. Goennenwein, M. Opel, R. Gross, and M. S. Brandt, 2006, "Spin-glasslike behavior of Ge:Mn," Phys. Rev. B 74, 045330

Jain, A., M. Jamet, A. Barski, T. Devillers, C. Porret, P. Bayle-Guillemaud, S. Gambarelli, V. Maurel, and G. Desfonds, 2010, "Investigation of magnetic anisotropy of (ge,mn) nanocolumns," Appl. Phys. Lett. 97, 202502

Jain, A., M. Jamet, A. Barski, T. Devillers, I.-S. Yu, C. Porret, P. Bayle-Guillemaud, V. Favre-Nicolin, S. Gambarelli, V. Maurel, G. Desfonds, J.-F. Jacquot, and S. Tardif, 2011, "Structure and magnetism of ge $\mathrm{mn}_{5}$ clusters," J. Appl. Phys. 109, 013911

Jamet, M., 2010 unpublished

Jamet, M., A. Barski, T. Devillers, V. Poydenot, R. Dujardin, P. Bayle-Guillemaud, J. Rothman, E. Bellet-Amalric, A. Marty, J. Cibert, R. Mattana, and S. Tatarenko, 2006, "High-Curie-temperature ferromagnetism in self-organized $\mathrm{Ge}_{1-x} \mathrm{Mn}_{x}$ nanocolumns," Nat. Mater. 5, 653

Jamet, M., W. Wernsdorfer, C. Thirion, D. Mailly, V. Dupuis, P. Mélinon, and A. Pérez, 2001, "Magnetic anisotropy of a single cobalt nanocluster," Phys. Rev. Lett. 86, 4676 
Jones, R. A. L., 2002, in Soft Condensed Matter (Oxford University Press, Oxford)

Jungwirth, T., J. Wunderlich, V. Novák, K. Olejník, B. L. Gallagher, R. P. Campion, K. W. Edmonds, A. W. Rushforth, A. J. Ferguson, and P. Němec, 2014, "Spin-dependent phenomena and device concepts explored in (Ga,Mn)As," Rev. Mod. Phys. 86, 855-896

Kacman, P., 2001, "Spin interactions in diluted magnetic semiconductors and magnetic semiconductor structures," Semicond. Sci. Technol. 16, R25

Kane, M. H., S. Gupta, W. E. Fenwick, N. Li, E.-H.Park, M. Strassburg, and I. T. Ferguson, 2007, "Comparative study of $\mathrm{Mn}$ and Fe incorporation into GaN by metalorganic chemical vapor deposition," phys. ptat. sol. (a) 204, 61

Kang, J. S., G. Kim, S. C. Wi, S. S. Lee, S. Choi, Sunglae Cho, S. W. Han, K. H. Kim, H. J. Song, H. J. Shin, A. Sekiyama, S. Kasai, S. Suga, and B. I. Min, 2005, "Spatial chemical inhomogeneity and local electronic structure of Mn-doped Ge ferromagnetic semiconductors," Phys. Rev. Lett. 94, 147202

Karczewski, G., M. Sawicki, V. Ivanov, C. Ruester, G. Grabecki, F. Matsukura, L.W. Molenkamp, and T. Dietl, 2003, "Ferromagnetism in (Zn,Cr)Se layers grown by molecular beam epitaxy," J. Supercond. Nov. Magn. 16, 55

Kashiwagi, T., S. Sonoda, H. Yashiro, Y. Akasaka, and M. Hagiwara, 2007, "ESR in a GaN thin film doped with Fe," J. Mag. Mag. Mat. 310, 2152

Katayama-Yoshida, H., K. Sato, T. Fukushima, M. Toyoda, H. Kizaki, V. A.Dinh, and P. H. Dederichs, 2007a, "Theory of ferromagnetic semiconductors," phys.stat. sol. (a) 204, 15

Katayama-Yoshida, H., K. Sato, T. Fukushima, M. Toyoda, H. Kizaki, V. A. Dinh, and P. H. Dederichs, 2007b, "Computational nano-materials design for high-Tc ferromagnetism in wide-gap magnetic semiconductors," J. Magn. Magn. Mater. 310, 2070

Katz, M. B., G. W. Graham, Yingwen Duan, Hong Liu, C. Adamo, D. G. Schlom, and Xiaoqing Pan, 2011, "Selfregeneration of $\mathrm{PdLaFeO}_{3}$ catalysts: New insight from atomic-resolution electron microscopy," J. Am. Chem. Soc. 133, 18090

Kawasaki, J.K., B.D. Schultz, Hong Lu, A.C. Gossard, and C.J. Palmstrøm, 2013, "Surface-mediated tunable self-assembly of single crystal semimetallic ErSb/GaSb nanocomposite structures," Nano Lett. 13, 2895

Kazakova, O., J. S. Kulkarni, J. D. Holmes, and S. O. Demokritov, 2005, "Room-temperature ferromagnetism in $\mathrm{Ge}_{1-x} \mathrm{Mn}_{x}$ nanowires," Phys. Rev. B 72, 094415

Keavney, D. J., S. H. Cheung, S. T. King, M. Weinert, and L. Li, 2005, "Role of defect sites and Ga polarization in the magnetism of Mn-Doped GaN," Phys. Rev. Lett. 95, 257201

Kenmochi, K., V. A. Dinh, K. Sato, A. Yanase, and H. Katayama-Yoshida, 2004a, "Materials design of transparent and half-metallic ferromagnets of $\mathrm{MgO}, \mathrm{SrO}$ and $\mathrm{BaO}$ without magnetic elements," J. Phys. Soc. Jap. 73, 2952

Kenmochi, K., K. Sato, A. Yanase, and H. KatayamaYoshida, 2005, "Materials design of ferromagnetic diamond," Jpn. J. Appl. Phys. 44, L51

Kenmochi, K., M. Seike, K. Sato, A. Yanase, and H. Katayama-Yoshida, 2004b, "New class of diluted ferro- magnetic semiconductors based on $\mathrm{CaO}$ without transition metal elements," Jpn. J. Appl. Phys. 43, L934

Khodaparast, G. A., Y. H. Matsuda, D. Saha, G. D. Sanders, C. J. Stanton, H. Saito, S. Takeyama, T. R. Merritt, C. Feeser, B. W. Wessels, X. Liu, and J. Furdyna, 2013, "Cyclotron resonance in ferromagnetic InMnAs and InMnSb," Phys. Rev. B 88, 235204

Kilanski, L., M. Górska, W. Dobrowolski, E. Dynowska, M. Wójcik, B. J. Kowalski, J. R. Anderson, C. R. Rotundu, D. K. Maude, S. A. Varnavskiy, I. V. Fedorchenko, and S. F. Marenkin, 2010, "Magnetism and magnetotransport of strongly disordered $\mathrm{Zn}_{1-x} \mathrm{Mn}_{x} \mathrm{GeAs}_{2}$ semiconductor: The role of nanoscale magnetic clusters," J. Appl. Phys. 108 (7), 073925

Kim, G., S. C. Wi, S. S. Lee, J.-S. Kang, S. Y. Choi, S. Cho, S. W. Han, K. H. Kim, H. J. Song, and H. J. Shin, 2005, "Scanning photoelectron microscopy study of $\mathrm{Ge}_{1-x} \mathrm{~T}_{x}(\mathrm{~T}$ $=\mathrm{Cr}, \mathrm{Fe}$ ) diluted ferromagnetic semiconductor single crystals," J. Appl. Phys. 97, 10A307

Kim, K. H., K. J. Lee, D. J. Kim, H. J. Kim, Y. E. Ihm, D. Djayaprawira, M. Takahashi, C. S. Kim, C. G. Kim, and S. H. Yoo, 2003, "Magnetotransport of $p$-type GaMnN assisted by highly conductive precipitates," Appl. Phys. Lett. 82, 1775

Kioseoglou, G., A. T. Hanbicki, C. H. Li, S. C. Erwin, R. Goswami, and B. T. Jonker, 2004, "Epitaxial growth of the diluted magnetic semiconductors $\mathrm{Cr}_{y} \mathrm{Ge}_{1-y}$ and $\mathrm{Cr}_{y} \mathrm{Mn}_{x} \mathrm{Ge}_{1-x-y}$," Appl. Phys. Lett. 84, 1725

Kizaki, H., and H. Katayama-Yoshida, 2013, "Spinodal nano decomposition in perovskite three-way catalysts: Firstprinciples calculations and Monte Carlo simulations," Chem. Phys. Lett. 579, 85

Kizaki, H., K. Kusakabe, S. Nogami, and H. KatayamaYoshida, 2008, "Generation of nano-catalyst particles by spinodal nano-decomposition in perovskite," Appl. Phys. Exp. 1, 104001

Kobayashi, H., Y. Nishio, K. Kanazawa, S. Kuroda, M. Mitome, and Y. Bando, 2012, "Structural analysis of the phase separation in magnetic semiconductor (Zn,Cr)Te," Physica B 407, 2947

Kobayashi, H., K. Yamawaki, Y. Nisho, K. Kanazawa, S. Kuroda, M. Mitome, and Y. Bando, 2013, "Structural analysis of $\mathrm{Cr}$ aggregation in ferromagnetic semiconductor (Zn,Cr)Te," Physics of Semiconductors, AIP Conf. Proc. 1566, 341

Kobayashi, M., Y. Ishida, J. I. Hwang, G. S. Song, C. S. Fujimori, A. Yang, Lee. L., H.-J. Lin, D. J. Huang, C. T. Chen, Y. Takeda, K. Terai, S.-I. Fujimori, T. Okane, Y. Saitoh, H. Yamagami, K. Kobayashi, A. Tanaka, H. Saito, and K. Ando, 2008, "Local electronic structure of $\mathrm{Cr}$ in the II-VI diluted ferromagnetic semiconductor $\mathrm{Zn}_{1-x} \mathrm{Cr}_{x} \mathrm{Te}$," New J. Phys. 10, 055011

Kocan, M., J. Malindretos, M. Roever, J. Zenneck, T. Niermann, D. Mai, M. Bertelli, M. Seibt, and A. Rizzi, 2006, "Mn incorporation in GaN thin layers grown by molecularbeam epitaxy," Semicond. Sci. Technol. 21, 1348

Kodzuka, M., 2012, Application of atom probe to microstructure analysis of thin film materials (PhD Thesis, Universisity of Tsukuba)

Kohn, W., and L. Sham, 1965, "Self-consistent equations including exchange and correlation effects," Phys. Rev. 140, A1133

Kondo, T., S. Kuwabara, H. Owa, and H. Munekata, 2002, "Molecular beam epitaxy of (Ga,Mn)N," J. Cryst. Growth 
237, 1353

Korenev, V.L., M. Salewski, I.A. Akimov, V.F. Sapega, L. Langer, I.V. Kalitukha, J. Debus, R.I. Dzhioev, D.R. Yakovlev, D. Mueller, C. Schroeder, H. Hoevel, G. Karczewski, M. Wiater, T. Wojtowicz, Yu.G. Kusrayev, and M. Bayer, 2015, "Long-range p-d exchange interaction in a ferromagnet-semiconductor hybrid structure," Nat. Phys. (doi 10.1038/NPHYS3497)

Kossacki, P., W. Pacuski, W. Maślana, J. A. Gaj, M. Bertolini, D. Ferrand, S. Tatarenko, and J. Cibert, 2004, "Spin engineering of carrier-induced magnetic ordering in (Cd,Mn)Te quantum wells," Physica E (Amsterdam) 21, 943

Kovàcs, A., T. Kasama, J.Sadowski, T. Dietl, and R. E. Dunin-Borkowski, 2011, "Aberration-corrected electron microscopy of MnAs and As nanocrystals and voids in annealed (Ga,Mn)As," J. Phys. Conference Series 326, 012018

Kowalik, I. A., A. Persson, M. Á. Niño, A. Navarro-Quezada, B. Faina, A. Bonanni, T. Dietl, and D. Arvanitis, 2012, "Element-specific characterization of heterogeneous magnetism in (Ga,Fe)N films," Phys. Rev. B 85, 184411

Krug von Nidda, H.-A., T. Kurz, A. Loidl, Th. Hartmann, P. J. Klar, W. Heimbrodt, M. Lampalzer, K. Volz, and W. Stolz, 2006, "Tuning the magnetic properties of GaAs:Mn/MnAs hybrids via the MnAs cluster shape," J. Phys. Cond. Matter 18, 6071

Kubota, M., T. Onuma, Y. Ishihara, A. Usui, A. Uedono, and S. F. Chichibu, 2009, "Thermal stability of semi-insulating property of Fe-doped GaN bulk films studied by photoluminescence and monoenergetic positron annihilation techniques," J. Appl. Phys. 105, 083542

Kudrin, A. V., A. V. Shvetsov, Yu. A. Danilov, A. A. Timopheev, D. A. Pavlov, A. I. Bobrov, N. V. Malekhonova, and N. A. Sobolev, 2014, "Anomalous hall effect in twophase semiconductor structures: The role of ferromagnetic inclusions," Phys. Rev. B 90, 024415

Kunert, G., S. Dobkowska, Tian Li, H. Reuther, C. Kruse, S. Figge, R. Jakieła, A. Bonanni, J. Grenzer, W. Stefanowicz, J. von Borany, M. Sawicki, T. Dietl, and D. Hommel, 2012, " $\mathrm{Ga}_{1-x} \mathrm{Mn}_{x} \mathrm{~N}$ epitaxial films with high magnetization," Appl. Phys. Lett. 101, 022413

Kuroda, S., N. Nishizawa, T. Kumekawa, S. Marcet, and K. Takita, 2005, "Growth and magnetic properties of novel ferromagnetic semiconductor (Zn,Cr)Te," Sci. Technol. Adv. Mater. 6, 558

Kuroda, S., N. Nishizawa, K. Takita, M. Mitome, Y. Bando, K. Osuch, and T. Dietl, 2007, "Origin and control of high temperature ferromagnetism in semiconductors," Nat. Mater. 6, 440

Kuwabara, S., K. Ishii, S. Haneda, T. Kondo, and H. Munekata, 2001a, "Preparation of wurtzite GaN-based magnetic alloy semiconductors by molecular beam epitaxy," Physica E 10, 233

Kuwabara, S., T. Kondo, T. Chikyow, P. Ahmet, and H. Munekata, 2001b, "Molecular beam epitaxy of wurtzite GaN-based magnetic alloy semiconductors," Jap. J. Appl. Phys. 40, L724

Kuzemsky, A. L., 2013, "Unconventional and exotic magnetism in carbon-based structures and related materials," Int. J. Mod. Phys. B 27, 1330007

Kwiatkowski, A., D. Wasik, M. Kamińska, R. Bożek, J. Szczytko, A. Twardowski, J. Borysiuk, J. Sadowski, and J. Gosk, 2007, "Structure and magnetism of MnAs nanocrystals embedded in GaAs as a function of postgrowth annealing temperature," J. Appl. Phys. 101, 113912

Lampalzer, M., S. Nau, C. Pietzonka, W. Treutmann, K. Volz, and W. Stolz, 2004, "Te-co-doping experiments in ferromagnetic $\mathrm{Mn}(\mathrm{Ga}) \mathrm{As} / \mathrm{GaAs}$ cluster hybrid layers by MOVPE," J. Crys. Growth 272, 772

Landau, D. P., and K. Binder, 2000, in A Guide to Monte Carlo Simulations in Statistical Physics (Cambridge University Press, Cambridge)

Langer, J. M., and H. Heinrich, 1985, "Deep-level impurities: A possible guide to prediction of band-edge discontinuities in semiconductor heterojunctions," Phys. Rev. Lett. 55, 1414

Lari, L., S. Lea, C. Feeser, B. W. Wessels, and V. K. Lazarov, 2012, "Ferromagnetic InMnSb multi-phase films study by aberration-corrected (scanning) transmission electron microscopy," J. Appl. Phys. 111, 07C311

Larson, P., and S. Satpathy, 2007, "Effect of vacancies on ferromagnetism in GaN:Mn dilute magnetic semiconductors from first-principles," Phys. Rev. B 76, 245205

Lawniczak-Jablonska, K., J. Libera, A. Wolska, M. T. Klepka, P. Dluzewski, J. Sadowski, D. Wasik, A. Twardowski, A. Kwiatkowski, and K. Sato, 2011, "The source of room temperature ferromagnetism in granular GaMnAs layers with zinc blende clusters," phys. stat. sol. (RRL) 5, 62

Lazarov, V. K., S. H. Cheung, Y. Cui, L. Li, and M. Gajdardziska-Josifovska, 2008, "Role of $\mathrm{Mn}$ and $\mathrm{H}$ in formation of cubic and hexagonal GaMnN," Appl. Phys. Lett. 92, 101914

Le, T.-G., D. N. H. Nam, M.-T. Dau, T. K. P. Luong, N. V. Khiem, V. Le Thanh, L. Michez, and J. Derrien, 2011, "The effects of Mn concentration on structural and magnetic properties of $\mathrm{Ge}_{1-x} \mathrm{Mn}_{x}$ diluted magnetic semiconductors," J. Phys.: Conf. Ser. 292, 012012

Lechner, R. T., V. Holý, S. Ahlers, D. Bougeard, J. Stangl, A. Trampert, A. Navarro-Quezada, and G. Bauer, 2009, "Self-assembled $\mathrm{Mn}_{5} \mathrm{Ge}_{3}$ nanomagnets close to the surface and deep inside a $\mathrm{Ge}_{1-x} \mathrm{Mn}_{x}$ epilayer," Appl. Phys. Lett. 95, 023102

Lethanh, V., 2006 private communication

Li, A. P., C. Zeng, K. van Benthem, M. F. Chisholm, J. Shen, S. V. S. Nageswara Rao, S. K. Dixit, L. C. Feldman, A. G. Petukhov, M. Foygel, and H. H. Weitering, 2007, "Dopant segregation and giant magnetoresistance in manganesedoped germanium," Phys. Rev. B 75, 201201(R)

Li, Li, Y. Guo, X. Y. Cui, Rongkun Zheng, K. Ohtani, C. Kong, A. V. Ceguerra, M. P. Moody, J. D. Ye, H. H. Tan, C. Jagadish, Hui Liu, C. Stampfl, H. Ohno, S. P. Ringer, and F. Matsukura, 2012, "Magnetism of Co-doped $\mathrm{ZnO}$ epitaxially grown on a $\mathrm{ZnO}$ substrate," Phys. Rev. B 85, 174430

Liu, C., F. Yun, and H. Morkoç, 2005, "Ferromagnetism of $\mathrm{ZnO}$ and GaN: A review," J. Mater. Sci.: Mater. Electron. 16, 555

Lo, Ikai, J. K. Tsai, M. H. Gau, Y. L. Chen, Z. J. Chang, W. T. Wang, J. C. Chiang, K. R. Wang, Chun-Nan Chen, T. Aggerstam, and S. Lourdudoss, 2006, "Study of two-subband population in Fe-doped $\mathrm{Al}_{x} \mathrm{Ga}_{1-x} \mathrm{~N} / \mathrm{GaN}$ heterostructures by persistent photoconductivity effect," Phys. Rev. B 74, 245325

Loss, D., and D. P. DiVincenzo, 1998, "Quantum computation with quantum dots," Phys. Rev. A 57, 120

Lu, E., D. C. Ingram, A. R. Smith, J. W. Knepper, and F. Y. Yang, 2006, "Reconstruction control of magnetic proper- 
ties during epitaxial growth of ferromagnetic $\mathrm{Mn}_{3-\delta} \mathrm{Ga}$ on wurtzite GaN(0001)," Phys. Rev. Lett. 97, 146101

Lu, Hong, D.G. Ouellette, S. Preu, J.D. Watts, B. Zaks, P.G. Burke, M.S. Sherwin, and A.C. Gossard, 2014, "Selfassembled ErSb nanostructures with optical applications in infrared and terahertz," Nano Lett. 14, 1107

Mac, W., A. Twardowski, and M. Demianiuk, 1996, "s,p-d exchange interaction in Cr-based diluted magnetic semiconductors," Phys. Rev. B 54, 5528

Maccherozzi, F., M. Sperl, G. Panaccione, J. Minar, S. Polesya, H. Ebert, U. Würstbauer, M. Hochstrasser, G. Rossi, G. Woltersdorf, W. Wegscheider, and C. H. Back, 2008, "Evidence for a magnetic proximity effect up to room temperature at Fe/(Ga,Mn)As interfaces," Phys. Rev. Lett. 101, 267201

Mack, S., R. C. Myers, J. T. Heron, A. C. Gossard, and D. D. Awschalom, 2008, "Stoichiometric growth of high Curie temperature heavily alloyed GaMnAs," Appl. Phys. Lett. 92, 192502

Mahadevan, Priya, J. M. Osorio-Guillén, and A. Zunger, 2005, "Origin of transition metal clustering tendencies in GaAs based dilute magnetic semiconductors," Appl. Phys. Lett. 86, 172504

Makarova, T., 2010, "Nanomagnetism in otherwise nonmagnetic materials," in Handbook in Nanophysics: Principles and Methods (ERC Press, Boca Raton FL) p. 25.1

Makarova, T. L., B. Sundqvist, R. Höhne, P. Esquinazi, Y. Kopelevich, P. Scharff, V. Davydov, L. S. Kashevarova, and A. V. Rakhmanina, 2006, "Retraction: Magnetic carbon," Nature 440, 707

Malguth, E., A. Hoffmann, W. Gehlhoff, O. Gelhausen, M. R. Phillips, and X. Xu, 2006a, "Structural and electronic properties of $\mathrm{Fe}^{3+}$ and $\mathrm{Fe}^{2+}$ centers in GaN from optical and EPR experiments," Phys. Rev. B 74, 165202

Malguth, E., A. Hoffmann, and M. R. Phillips, 2008, "Fe in III-V and II-VI semiconductors," Phys. Status Solidi B 245, 455

Malguth, E., A. Hoffmann, and X. Xu, 2006b, "Internal ${ }^{5} e \rightarrow^{5}$ $t_{2}$ transition of $\mathrm{Fe}^{2+}$ in GaN," Phys. Rev. B 74, 165201

Martin, R. M., 2004, in Electronic structure (Cambridge University Press)

Martinez-Criado, G., A. Somogyi, S. Ramos, J. Campo, R. Tucoulou, M. Salome, J. Susini, M. Hermann, M. Eickhoff, and M. Stutzmann, 2005, "Mn-rich clusters in GaN: Hexagonal or cubic symmetry?." Appl. Phys. Lett. 86, 131927

Massalski, T. B., 1990, "Binary alloy phase diagrams," American Society for Metals, Metals Pair, OH, 19902

Matsubayashi, K., M. Maki, T. Tsuzuki, T. Nishioka, and N. K. Sato, 2002, "Parasitic ferromagnetism in a hexaboride?." Nature 420, 143

Matsukura, F., H. Ohno, and T. Dietl, 2002, "III-V ferromagnetic semiconductors," in Handbook of Magnetic Materials, Vol. 14, edited by K. H. J. Buschow (Elsevier) p. 1

Mavropoulos, P., M. Ležić, and S. Blügel, 2009, "Ferromagnetism in nitrogen-doped $\mathrm{MgO}$ : Density-functional calculations," Phys. Rev. B 80, 184403

van der Meulen, M. I., N. Petkov, M. A. Morris, O. Kazakova, X. Han, K. L. Wang, A. P. Jacob, and J. D. Holmes, 2009, "Single crystalline $\mathrm{Ge}_{1-x} \mathrm{Mn}_{x}$ nanowires as building blocks for nanoelectronic," Nano Lett. 9, 50

Miao, Guo-xing, Joonyeon Chang, Badih A. Assaf, D. Heiman, and J. S. Moodera, 2014, "Spin regulation in composite spin-filter barrier devices," Nat. Commun. 5,
3682

Miao, M. S., A. Herwadkar, and W. R. L. Lambrecht, 2005, "Electronic structure and magnetic properties of $\mathrm{Mn}_{3} \mathrm{GaN}$ precipitates in $\mathrm{Ga}_{1-x} \mathrm{Mn}_{x} \mathrm{~N}$," Phys. Rev. B 72, 033204

Michel, C., M. T. Elm, B. Goldlücke, S. D. Baranovskii, P. Thomas, W. Heimbrodt, and P. J. Klar, 2008, "Tailoring the magnetoresistance of MnAs/GaAs:Mn granular hybrid nanostructures," Appl. Phys. Lett. 92, 223119

Michel, C., C.H. Thien, S. Ye, P.J. Klar, W. Heimbrodt, S.D. Baranovskii, P. Thomas, M. Lampalzer, K. Volz, W. Stolz, and B. Goldlücke, 2005, "Spin-dependent localization effects in GaAs:Mn/MnAs granular paramagneticferromagnetic hybrids at low temperatures," Superlatt. Microstruct. 37, 321

Mirbt, S., B. Sanyal, and P. Mohn, 2002, "Magnetic properties of 3d impurities substituted in GaAs," J. Phys.: Condens. Matter 14, 3295

Mizokawa, T., and A. Fujimori, 1997, " $p-d$ exchange interaction for $3 d$ transition-metal impurities in II-VI semiconductors," Phys. Rev. B 56, 6669

Monette, G., C. Lacroix, S. Lambert-Milot, V. Boucher, D. Ménard, and S. Francoeur, 2010, "Giant magnetooptical Faraday effect in GaP epilayers containing MnP magnetic nanoclusters," J. Appl. Phys. 107, 09A949

Moreno, M., B. Jenichen, V. Kaganer, W. Braun, A. Trampert, L. Däweritz, and K.H. Ploog, 2003, "MnAs nanoclusters embedded in GaAs studied by x-ray diffuse and coherent scattering," Phys. Rev. B 67, 235206

Moreno, M., V. M. Kaganer, B. Jenichen, A. Trampert, L. Däweritz, and K. H. Ploog, 2005, "Micromechanics of MnAs nanocrystals embedded in GaAs," Phys. Rev. B 72, 115206

Moreno, M., A. Trampert, B. Jenichen, L. Däweritz, and K. H. Ploog, 2002, "Correlation of structure and magnetism in GaAs with embedded $\mathrm{Mn}(\mathrm{Ga})$ As magnetic nanoclusters," J. Appl. Phys. 92, 4672

Morresi, L., N. Pinto, M. Ficcadenti, R. Murri, F. D’Orazio, and F. Lucari, 2006, "Magnetic and transport polaron percolation in diluted GeMn films," Mater. Sci. Eng. B 126, 197

Mørup, S., M. F. Hansen, and C. Frandsen, 2010, "Magnetic interactions between nanoparticles," Beilstein J. Nanotechnol. 1, 182

Mouton, I., R. Lardé, E. Talbot, E. Cadel, C. Genevois, D. Blavette, V. Baltz, E. Prestat, P. Bayle Guillemaud, A. Barski, and M. Jamet, 2012, "Composition and morphology of self-organized Mn-rich nanocolumns embedded in Ge: Correlation with the magnetic properties," J. Appl. Phys. 112, 113918

Munekata, H., H. Ohno, S. von Molnar, A. Segmüller, L. L. Chang, and L. Esaki, 1989, "Diluted magnetic III-V semiconductors," Phys. Rev. Lett. 63, 1849

Muret, P., J. Pernot, M. Azize, and Z. Bourigoua, 2007, "Photoinduced current transient spectroscopy of deep levels and transport mechanisms in iron-doped GaN thin films grown by low pressure-metalorganic vapor phase epitaxy," J. Appl. Phys. 102, 053701

Navarro-Quezada, A., T. Devillers, Tian Li, and A. Bonanni, 2012, "Planar arrays of magnetic nanocrystals embedded in GaN," Appl. Phys. Lett. 101, 081911

Navarro-Quezada, A., N. Gonzalez Szwacki, W. Stefanowicz, Tian Li, A. Grois, T. Devillers, M. Rovezzi, R. Jakieła, B. Faina, J. A. Majewski, M. Sawicki, T. Dietl, and A. Bonanni, 2011, "Fe-Mg interplay and the effect of deposi- 
tion mode in (Ga,Fe)N doped with Mg," Phys. Rev. B 84, 155321

Navarro-Quezada, A., W. Stefanowicz, Tian Li, B. Faina, M. Rovezzi, R. T. Lechner, T. Devillers, F. d'Acapito, G. Bauer, M. Sawicki, T. Dietl, and A. Bonanni, 2010, "Embedded magnetic phases in $(\mathrm{Ga}, \mathrm{Fe}) \mathrm{N}$ : Key role of growth temperature," Phys. Rev. B 81, 205206

Nealon, G. L., B. Donnio, R. Greget, J.-P. Kappler, E. Terazzi, and J.-L. Gallani, 2012, "Magnetism in gold nanoparticles," Nanoscale 4, 5244

Ney, A., T. Kammermeier, V. Ney, K. Ollefs, and S. Ye, 2008, "Limitations of measuring small magnetic signals of samples deposited on a diamagnetic substrate," J. Magn. Magn. Mater. 320, 3341

Niida, H., T. Hori, H. Onodera, Y. Yamaguchi, and Y. Nakagawa, 1996, "Magnetization and coercivity of $\mathrm{Mn}_{3-\delta} \mathrm{Ga}$ alloys with a $\mathrm{D0}_{22}$-type structure," J. Appl. Phys. 79, 5946

Nishio, Yôtarô, Kôichirô Ishikawa, Shinji Kuroda, Masanori Mitome, and Yoshio Bando, 2009, "Formation of Crrich nano-clusters and columns in $(\mathrm{Zn}, \mathrm{Cr}) \mathrm{Te}$ grown by MBE," in Mater. Res. Soc. Symp. Proc., MRS Proceedings, Vol. 1183-FF01-11 (Materials Research Society, Warrendale PA) pp. FF01-11

Novick-Cohen, A., 2008, "The Cahn-Hilliard equation," in Evolutionary Equations, Handbook of Differential Equations, Vol. 4, edited by C.M. Dafermos and E. Feireisl, Chap. 4 (Elsevier, Amsterdam) p. 201

Ofuchi, H., K. Ishikawa, K. Zhang, S. Kuroda, M. Mitome, and Y. Bando, 2009, "Fluorescence XAFS analysis of local structures in iodine-doped $\mathrm{Zn}_{1-x} \mathrm{Cr}_{x}$ Te," J. Phys.: Conf. Ser. 190, 012103

Ofuchi, H., M. Oshima, M. Tabuchi, Y. Takeda, H. Akinaga, S. Németh, J. De Boeck, and G. Borghs, 2001, "Fluorescence x-ray absorption fine structure study on local structures around Fe atoms heavily doped in GaN by lowtemperature molecular-beam epitaxy," Appl. Phys. Lett. $\mathbf{7 8}, 2470$

Ohba, T., K. Kifune, and Y. Komura, 1987, "Structure determination of $\zeta_{2}-\mathrm{Mn}_{5} \mathrm{Ge}_{2}$ using a mixed crystal," Acta Cryst. B43, 489

Ohba, T., N. Watanabe, and Y. Komura, 1984, "Temperature dependence of the lattice constants and the structure of $\mathrm{Mn}_{11} \mathrm{Ge}_{8}$ at 295 and $116 \mathrm{k}$," Acta Cryst. B40, 351

Ohno, H., A. Shen, F. Matsukura, A. Oiwa, A. Endo, S. Katsumoto, and Y. Iye, 1996, "(Ga,Mn)As: A new diluted magnetic semiconductor based on GaAs," Appl. Phys. Lett. 69, 363

Ohoyama, T., 1961, "X-ray and magnetic studies of the manganese-germanium system," J. Phys. Soc. Jpn. 16, 1995

Ohoyama, T., K. Yasukochi, and K. Kanematsu, 1961, "A new phase of an intermetallic compound $\mathrm{Mn}_{3.4} \mathrm{Ge}$ and its magnetism," J. Phys. Soc. Jpn. 16, 352

Ohta, S., T. Kanomata, T. Kaneko, and H. Yoshida, 1993, "Pressure effect on the Curie temperature and thermal expansion of CrTe," J. Phys.: Condens. Matter 5, 2759

Ohya, S., K. Ohno, and M. Tanaka, 2007, "Magneto-optical and magnetotransport properties of heavily Mn-doped GaMnAs," Appl. Phys. Lett. 90, 112503

Oshitani, M., K. Sato, and H. Katayama-Yoshida, 2011, "Design of self-organized spinodal nano-decomposition for high-efficiency photovoltaic solar cells in II-VI compound semiconductors alloy," Appl. Phys. Exp. 4, 022302, erratum: Appl. Phys. Express 4, 049201
Ottaviano, L., M. Passacantando, A. Verna, F. D'Amico, and R. Gunnella, 2007, "Mn L $\mathrm{L}_{2,3}$ x-ray absorption spectra of a diluted Mn-Ge alloy," Appl. Phys. Lett. 90, 242105

Ottaviano, L., M. Passacantando, A. Verna, R. Gunnella, E. Principi, A. D. Cicco, G. Impellizzeri, and F. Priolo, 2006, "Direct structural evidences of Mn dilution in Ge," J. Appl. Phys. 100, 063528

Ozaki, N., N. Nishizawa, S. Marcet, S. Kuroda, O. Eryu, and K. Takita, 2006, "Significant enhancement of ferromagnetism in $\mathrm{Zn}_{1-x} \mathrm{Cr}_{x} \mathrm{Te}$ doped with iodine as an $n$-type dopant," Phys. Rev. Lett. 97, 037201

Ozaki, N., J. Okabayashi, T. Kumekawa, N. Nishizawa, S. Marcet, S. Kuroda, and K. Takita, 2005, "Suppression of ferromagnetism due to hole doping in $\mathrm{Zn}_{1-x} \mathrm{Cr}_{x}$ Te grown by molecular-beam epitaxy," Appl. Phys. Lett. 87, 192116

Pacuski, W., D. Ferrand, J. Cibert, C. Deparis, J. A. Gaj, P. Kossacki, and C. Morhain, 2006, "Effect of the s,p-d exchange interaction on the excitons in $\mathrm{Zn}_{1-x} \mathrm{Co}_{x} \mathrm{O}$ epilayers," Phys. Rev. B 73, 035214

Pacuski, W., D. Ferrand, J. Cibert, J. A. Gaj, A. Golnik, P. Kossacki, S. Marcet, E. Sarigiannidou, and H. Mariette, 2007, "Excitonic giant Zeeman effect in GaN:Mn ${ }^{3+}$," Phys. Rev. B 76, 165304

Pacuski, W., P. Kossacki, D. Ferrand, A. Golnik, J. Cibert, M. Wegscheider, A. Navarro-Quezada, A. Bonanni, M. Kiecana, M. Sawicki, and T. Dietl, 2008, "Observation of strong-coupling effects in a diluted magnetic semiconductor $\mathrm{Ga}_{1-x} \mathrm{Fe}_{x} \mathrm{~N}$," Phys. Rev. Lett. 100, 037204

Pacuski, W., J. Suffczyński, P. Osewski, P. Kossacki, A. Golnik, J. A. Gaj, C. Deparis, C. Morhain, E. Chikoidze, Y. Dumont, D. Ferrand, J. Cibert, and T. Dietl, 2011, "Influence of $s, p-d$ and $s-p$ exchange couplings on exciton splitting in $\mathrm{Zn}_{1-x} \mathrm{Mn}_{x} \mathrm{O}$," Phys. Rev. B 84, 035214

Padova, P. D., J.-P. Ayoub, I. Berbezier, J.-M. Mariot, A. Taleb-Ibrahimi, M. C. Richter, O. Heckmann, A. M. Testa, D. Fiorani, B. Olivieri, S. Picozzi, and K. Hricovini, 2007, " $\mathrm{Mn}_{x} \mathrm{Ge}_{1-x}$ thin layers studied by TEM, X-ray absorption spectroscopy and SQUID magnetometry," Surf. Sci. 601, 2628

Padova, P. D., J.-P. Ayoub, I. Berbezier, P. Perfetti, C. Quaresima, A. M. Testa, D. Fiorani, B. Olivieri, J.M. Mariot, A. Taleb-Ibrahimi, M. C. Richter, O. Heckmann, and K. ricovini, 2008, " $\mathrm{Mn}_{0.06} \mathrm{Ge}_{0.94}$ diluted magnetic semiconductor epitaxially grown on $\mathrm{Ge}(001)$ : Influence of $\mathrm{Mn}_{5} \mathrm{Ge}_{3}$ nanoscopic clusters on the electronic and magnetic properties," Phys. Rev. B 77, 045203

Pajączkowska, A., 1978, "Physicochemical properties and crystal growth of $\mathrm{A}^{I I} \mathrm{~B}^{V I}-\mathrm{MnB}^{V I}$ systems," Prog. Cryst. Growth Charact. 1, 289

Panov, A. V., 2012, "Dipolar ordering of random twodimensional spin ensemble," Appl. Phys. Lett. 100, 052406

Park, J., J. Cho, K. Nishimura, and M. Inoue, 2002a, "Magnetooptic spatial light modulator for volumetric digital recording system," Jpn. J. Appl. Phys. 41, 1813

Park, Y. D., A. T. Hanbicki, S. C. Erwin, C. S. Hellberg, J. M. Sullivan, J. E. Mattson, T. F. Ambrose, A. Wilson, G. Spanos, and B. T. Jonker, 2002b, "A group-IV ferromagnetic semiconductor: $\mathrm{Mn}_{x} \mathrm{Ge}_{1-x}$," Science 295, 651

Park, Y. D., A. Wilson, A. T. Hanbicki, J. E. Mattson, T. Ambrose, G. Spanos, and B. T. Jonker, 2001, "Magnetoresistance of $\mathrm{Mn}$ :Ge ferromagnetic nanoclusters in a diluted magnetic semiconductor matrix," Appl. Phys. Lett. 78, 2739

Passacantando, M., L. Ottaviano, F. D'Orazio, F. Lucari, 
M. De Biase, G. Impellizzeri, and F. Priolo, 2006, "Growth of ferromagnetic nanoparticles in a diluted magnetic semiconductor obtained by mn+ implantation on ge single crystals," Phys. Rev. B 73, 195207

Pearton, S. J., C. R. Abernathy, M. E. Overberg, G. T. Thaler, D. P. Norton, N. Theodoropoulou, A. F. Hebard, Y. D. Park, F. Ren, J. Kim, and L. A. Boatner, 2003, "Wide band gap ferromagnetic semiconductors and oxides," J. Appl. Phys. 93, 1

Pearton, S. J., M. E. Overberg, G. Thaler, C. R. Abernathy, N. Theodoropoulou, A. F. Hebard, S. N. G. Chu, R. G. Wilson, J. M. Zavada, A. Y. Polyakov, A. V. Osinsky, P. E. Norris, P. P. Chow, A. M. Wowchack, J. M. Van Hove, and Y. D. Park, 2002, "Characterization of high dose Mn, Fe and Ni implantation into p-GaN," J. Vac. Sci. Technol. A 20, 721

Perdew, J. P., and Y. Wang, 1986, "Accurate and simple density functional for the electronic exchange energy: Generalized gradient approximation," Phys. Rev. 33, 8800

Pinto, N., L. Morresi, M. Ficcadenti, R. Murri, F. D'Orazio, F. Lucari, L. Boarino, and G. Amato, 2005, "Magnetic and electronic transport percolation in epitaxial $\mathrm{Ge}_{1-x} \mathrm{Mn}_{x}$ films," Phys. Rev. B 72, 165203

Pioro-Ladrière, M., T. Obata, Y. Tokura, Y.-S. Shin, T. Kubo, K. Yoshida, T. Taniyama, and S. Tarucha, 2008, "Electrically driven single-electron spin resonance in a slanting Zeeman field," Nat. Phys. 4, 776

Piskorska-Hommel, E., M. J. Winiarski, G. Kunert, I. N. Demchenko, O. D. Roshchupkina, J. Grenzer, J. Falta, D. Hommel, and V. Holý, 2015, "The electronic structure of homogeneous ferromagnetic (Ga,Mn)N epitaxial films," J. Appl. Phys. 117, 065702

Pop, I., M. Andrecut, I. Burda, R. Munteanu, and K. Criveanu, 1994, "Itinerant ferromagnetism in $\mathrm{Mn}_{4} \mathrm{~N}$," Mater. Chem. Phys. 37, 52

Przeździecka, E., E. Kamińska, M. Kiecana, M. Sawicki, Ł. Kłopotowski, W. Pacuski, and J. Kossut, 2006, "Magneto-optical properties of the diluted magnetic semiconductor $p$-type ZnMnO," Solid State Commun. 139, 541

Przybylińska, H., A. Bonanni, A. Wolos, M. Kiecana, M. Sawicki, T. Dietl, H. Malissa, C. Simbrunner, M. Wegscheider, H. Sitter, K. Rumpf, P. Granitzer, H. Krenn, and W. Jantsch, 2006, "Magnetic properties of a new spintronic material-GaN:Fe," Mater. Sci. Engineer. B 126, 222

Qi, Y., G. F. Sun, M. Weinert, and L. Li, 2009, "Electronic structures of Mn-induced phases on GaN(0001)," Phys. Rev. B 80, 235323

Raebiger, H., H. Nakayama, and T. Fujita, 2014, "Control of defect binding and magnetic interaction energies in dilute magnetic semiconductors by charge state manipulation," J. Appl. Phys. 115, 012008

Ralph, D. C., 2011, "The electromotive force of MnAs nanoparticles," Nature 474, E6

Reed, M. J., F. E. Arkun, E. A. Berkman, N. A. Elmasry, J. Zavada, M. O. Luen, M. L. Reed, and S. M. Bedair, 2005, "Effect of doping on the magnetic properties of GaMnN: Fermi level engineering," Appl. Phys. Lett. 86, 102504

Reiss, G., and D. Meyners, 2006, "Reliability of field programmable magnetic logic gate arrays," Appl. Phys. Lett. 88, 043505

Rench, D. W., P. Schiffer, and N. Samarth, 2011, "Structural and magnetic characteristics of MnAs nanoclusters embedded in Be-doped GaAs," Phys. Rev. B 84, 094434

Roever, M., J. Malindretos, A. Bedoya-Pinto, A. Rizzi, Ch.
Rauch, and F. Tuomisto, 2011, "Tracking defect-induced ferromagnetism in GaN:Gd," Phys. Rev. B 84, 081201

Rousset, J.-G., J. Papierska, W. Pacuski, A. Golnik, M. Nawrocki, W. Stefanowicz, S. Stefanowicz, M. Sawicki, R. Jakieła, T. Dietl, A. Navarro-Quezada, B. Faina, T. Li, A. Bonanni, and J. Suffczyński, 2013, "Relation between exciton splittings, magnetic circular dichroism, and magnetization in wurtzite $\mathrm{Ga}_{1-x} \mathrm{Fe}_{x} \mathrm{~N}$," Phys. Rev. B 88, 115208

Rovezzi, M., F. D'Acapito, A. Navarro-Quezada, B. Faina, Tian Li, A. Bonanni, F. Filippone, A. A. Bonapasta, and T. Dietl, 2009, "Local structure of $(\mathrm{Ga}, \mathrm{Fe}) \mathrm{N}$ and (Ga,Fe)N:Si investigated by x-ray absorption fine structure spectroscopy," Phys. Rev. B 79, 195209

Rovezzi, M., T. Devillers, E. Arras, F. d'Acapito, A. Barski, M. Jamet, and P. Pochet, 2008, "Atomic structure of Mn-rich nanocolumns probed by x-ray absorption spectroscopy," Appl. Phys. Lett. 92, 242510

Rubinstein, M., and R. H. Colby, 2003, in Polymer Physics (Oxford University Press, Oxford)

Rylkov, V. V., S. N. Nikolaev, K. Yu. Chernoglazov, B. A. Aronzon, K. I. Maslakov, V. V. Tugushev, E. T. Kulatov, I. A. Likhachev, E. M. Pashaev, A. S. Semisalov, N. S. Perov, A. B. Granovskii, E. A. Ganshina, O. A. Novodvorskii, O. D. Khramova, E. V. Khaidukov, and V. Ya. Panchenko, 2012, "High-temperature ferromagnetism in $\mathrm{Si}_{1-x} \mathrm{Mn}_{x}(x \approx 0.5)$ nonstoichiometric alloys," JETP Lett. 96, 255

Sadowski, J., J. Z. Domagala, R. Mathieu, A. Kovacs, and P. Dłużewski, 2013, "Formation of two-dimensionally confined superparamagnetic (Mn, Ga) As nanocrystals in hightemperature annealed (Ga, Mn)As/GaAs superlattices," J. Phys.: Cond. Matter 25, 196005

Sadowski, J., J. Z. Domagala, R. Mathieu, A. Kovács, T. Kasama, R. E. Dunin-Borkowski, and T. Dietl, 2011, "Formation process and superparamagnetic properties of (Mn,Ga)As nanocrystals in GaAs fabricated by annealing of (Ga,Mn)As layers with low Mn content," Phys. Rev. B $\mathbf{8 4}, 245306$

Saito, H., V. Zayets, S. Yamagata, and K. Ando, 2002, "Magneto-optical studies of ferromagnetism in the II-VI diluted magnetic semiconductor $\mathrm{Zn}_{1-x} \mathrm{Cr}_{x} \mathrm{Te}$," Phys. Rev. B 66, 081201

Saito, H., V. Zayets, S. Yamagata, and K. Ando, 2003, "Room-temperature ferromagnetism in a II-VI diluted magnetic semiconductor $\mathrm{Zn}_{1-x} \mathrm{Cr}_{x}$ Te," Phys. Rev. Lett. 90, 207202

Salas, R., S. Guchhait, S. D. Sifferman, K. M. McNicholas, V. D. Dasika, E. M. Krivoy, D. Jung, M. L. Lee, and S. R. Bank, 2015, "Growth and properties of rare-earth arsenide InGaAs nanocomposites for terahertz generation," Appl. Phys. Lett. 106, 081103

Sanyal, B., O. Bengone, and S. Mirbt, 2003, "Electronic structure and magnetism of Mn-doped GaN," Phys. Rev. B 68, 205210

Sarigiannidou, E., F. Wilhelm, E. Monroy, R. M. Galera, E. Bellet-Amalric, A.Rogalev, J. Goulon, J. Cibert, and H. Mariette, 2006, "Intrinsic ferromagnetism in wurtzite (Ga,Mn)N semiconductor," Phys. Rev. B 74, 041306(R)

Sato, K., L. Bergqvist, J. Kudrnovský, P. H. Dederichs, O. Eriksson, I. Turek, B. Sanyal, G. Bouzerar, H. Katayama-Yoshida, V. A. Dinh, T. Fukushima, H. Kizaki, and R. Zeller, 2010, "First-principles theory of dilute magnetic semiconductors," Rev. Mod. Phys. 82, 1633 
Sato, K., T. Fukushima, and H. Katayama-Yoshida, 2007, "Super-paramagnetic blocking phenomena and roomtemperature ferromagnetism in wide band-gap dilute magnetic semiconductor (Ga,Mn)N," Jpn. J. Appl. Phys. 46 L682

Sato, K., and H. Katayama-Yoshida, 2002, "First principles materials design for semiconductor spintronics," Semicond. Sci. Technol. 17, 367

Sato, K., and H. Katayama-Yoshida, 2007, "Design of colossal solubility of magnetic impurities for semiconductor spintronics by the Co-doping method," Jpn. J. Appl. Phys. 46, L1120

Sato, K., H. Katayama-Yoshida, and P. H. Dederichs, 2005, "High Curie temperature and nano-scale spinodal decomposition phase in diluted magnetic semiconductors," Jpn. J. Appl. Phys. 44, L948

Sato, M., H. Tanida, K. Kato, T. Sasaki, Y. Yamamoto, S. Sonoda, S. Shimizu, and H. Hori, 2002, "Local structure around $\mathrm{Mn}$ in ferromagnetic GaMnN film studied by X-ray absorption fine structure," Jap. J. Appl. Phys. 41, 4513

Sawicki, M., T. Devillers, S. Gałęski, C. Simserides, S. Dobkowska, B. Faina, A. Grois, A. Navarro-Quezada, K. N. Trohidou, J. A. Majewski, T. Dietl, and A. Bonanni, 2012, "Origin of low-temperature magnetic ordering in $\mathrm{Ga}_{1-x} \mathrm{Mn}_{x} \mathrm{~N}$," Phys. Rev. B 85, 205204

Sawicki, M., E. Guziewicz, M. I. Łukasiewicz, O. Proselkov, I. A. Kowalik, W. Lisowski, P. Dluzewski, A. Wittlin, M. Jaworski, A. Wolska, W. Paszkowicz, R. Jakiela, B. S. Witkowski, L. Wachnicki, M. T. Klepka, F. J. Luque, D. Arvanitis, J. W. Sobczak, M. Krawczyk, A. Jablonski, W. Stefanowicz, D. Sztenkiel, M. Godlewski, and T. Dietl, 2013, "Homogeneous and heterogeneous magnetism in $(\mathrm{Zn}, \mathrm{Co}) \mathrm{O}$ : From a random antiferromagnet to a dipolar superferromagnet by changing the growth temperature," Phys. Rev. B 88, 085204

Sawicki, M., W. Stefanowicz, and A. Ney, 2011, "Sensitive SQUID magnetometry for studying nanomagnetism," Semicon. Sci. Technol. 26, 064006

Schulthess, T. C., and W. H. Butler, 2001, "Electronic structure and magnetic interactions in Mn doped semiconductors," J. Appl. Phys. 89, 7021

Seike, M., V. A. Dinh, T. Fukushima, K. Sato, and H. Katayama-Yoshida, 2012, "Self-organized nanostructures and high blocking temperatures in $\mathrm{MgO}$-based $\mathrm{d}^{0}$ ferromagnets," Jap. J. Appl. Phys. 51, 050201

Seike, M., T. Fukushima, K. Sato, and H. Katayama-Yoshida, 2013a, "Design of self-organized nanostructures to achieve high blocking temperatures in $\mathrm{MgO}$-based $\mathrm{d}^{0}$ ferromagnets," J. Korean Phys. Soc. 62, 1807

Seike, M., T. Fukushima, K. Sato, and H. Katayama-Yoshida, 2013b, "Self-organized ferromagnetic nanowires in $\mathrm{MgO}-$ based magnetic tunnel junctions," Solid State Commun. 167, 14

Shi, Jing, S. Gider, K. Babcock, and D. D. Awschalom, 1996, "Magnetic clusters in molecular beams, metals, and semiconductors," Science 271, 937

Shiba, H., 1971, "A reformulation of the coherent potential approximation and its applications," Prog. Theor. Phys. 46, 77

Shick, A. B., S. Khmelevskyi, O. N. Mryasov, J. Wunderlich, and T. Jungwirth, 2010, "Spin-orbit coupling induced anisotropy effects in bimetallic antiferromagnets: A route towards antiferromagnetic spintronics," Phys. Rev. B 81,
212409

Shimada, K., T. Saitoh, H. Namatame, A. Fujimori, S. Ishida, S. Asano, M. Matoba, and S. Anzai, 1996, "Photoemission study of itinerant ferromagnet $\mathrm{Cr}_{1-\delta}$ Te," Phys. Rev. B 53, 7673

Shimizu, H., M. Miyamura, and M. Tanaka, 2000, "Enhanced magneto-optical effect in a GaAs:MnAs nanoscale hybrid structure combined with GaAs/AlAs distributed Bragg reflectors," J. Vacuum Sci. Techn. B 18, 2063

Shimizu, H., M. Miyamura, and M. Tanaka, 2001, "Magnetooptical properties of a GaAs:MnAs hybrid structure sandwiched by GaAs/AlAs distributed Bragg reflectors: Enhanced magneto-optical effect and theoretical analysis," Appl. Phys. Lett. 78, 1523

Shimizu, H., and M. Tanaka, 2001, "Magneto-optical properties of semiconductor-based superlattices having GaAs with MnAs nanoclusters," J. Appl. Phys. 89, 7281

Shimizu, H., and M. Tanaka, 2002a, "Design of semiconductor-waveguide-type optical isolators using the nonreciprocal loss/gain in the magneto-optical waveguides having MnAs nanoclusters," Appl. Phys. Lett. 81, 5246

Shimizu, H., and M. Tanaka, 2002b, "Magneto-optical properties of a Si-doped GaAs:MnAs-based magneto-photonic crystal operating at $1.55 \mu \mathrm{m}$," Physica E 13, 597

Shinya, H., A. Masago, T. Fukushima, H. Funashima, and H. Katayama-Yoshida, 2014, "First-principles investigations of defect and phase stabilities in thermoelectric $(\mathrm{GeTe})_{x}(\mathrm{AgSbTe} 2)_{1-x}, "$ Jpn. J. Appl. Phys. 53, 111201

Shon, Y., Y. Hae Kwon, Y. S. Park, Sh. U. Yuldashev, Seung Joo Lee, C. S. Park, K. J. Chung, S. J. Yoon, H. J. Kim, W. C. Lee, D. J. Fu, T. W. Kangb, X. J. Fan, Y. J. Park, and H. T. Oh, 2004, "Ferromagnetic behavior of $p$ type GaN epilayer implanted with $\mathrm{Fe}^{+}$ions," J. Appl. Phys. 95, 761

Shon, Y., S. Lee, H. C. Jeon, Y. S. Park, D. Y. Kim, T. W. Kang, J. S. Kim, E. K. Kim, D. J. Fu, X. J. Fan, Y. J. Park, J. M. Baik, and J. L. Lee, 2006, "Origin of clear ferromagnetism for $p$-type $\mathrm{GaN}$ implanted with $\mathrm{Fe}^{+}(5$ and 10 at.\%)," Appl. Phys. Lett. 89, 082505

Shuto, Y., M. Tanaka, and S. Sugahara, 2006a, "Epitaxial growth and magnetic properties of a new group-IV ferromagnetic semiconductor: $\mathrm{Ge}_{1-x} \mathrm{Fe}_{x}$," phys. stat. solidi (c) 3, 4110

Shuto, Y., M. Tanaka, and S. Sugahara, 2006b, "Magnetooptical properties of group-IV ferromagnetic semiconductor $\mathrm{Ge}_{1-x} \mathrm{Fe}_{x}$ grown by low-temperature molecular beam epitaxy," J. Appl. Phys. 99, 08D516

Shuto, Y., M. Tanaka, and S. Sugahara, 2007, "Structural and magnetic properties of epitaxially grown $\mathrm{Ge}_{1-x} \mathrm{Fe}_{x}$ thin films: Fe concentration dependence," Appl. Phys. Lett. 90, 132512

Smit, J., and H. G. Beljers, 1955, "Ferromagnetic resonance absorption in $\mathrm{BaFe}_{12} \mathrm{O}_{19}$, a high anisotropy crystal," Phillips Res. Rep. 10, 113

Solin, S. A., T. Thio, D.R. Hines, and J. J. Heremans, 2000, "Enhanced room-temperature geometric magnetoresistance in inhomogeneous narrow-gap semiconductors," Science 289, 1530

Sonoda, S., I. Tanaka, F. Oba, H. Ikeno, H. Hayashi, T. Yamamoto, Y. Yuba, Y. Akasaka, K. Yoshida, M. Aoki, M. Asari, T. Araki, Y. Nanishi, K. Kindo, and H. Hori, 2007, "Awaking of ferromagnetism in GaMnN through control of Mn valence," Appl. Phys. Lett. 90, 012504 
Stangl, J., V. Holý, and G. Bauer, 2004, "Structural properties of self-organized semiconductor nanostructures," Rev. Mod. Phys. 76, 725

Stauffer, D., and A. Aharony, 1994, in Introduction to percolation theory (revised 2nd el.) (Taylor and Francis, 1994)

Stefanowicz, S., G. Kunert, C. Simserides, J. A. Majewski, W. Stefanowicz, C. Kruse, S. Figge, Tian Li, R. Jakieła, K. N. Trohidou, A. Bonanni, D. Hommel, M. Sawicki, and T. Dietl, 2013, "Phase diagram and critical behavior of a random ferromagnet $\mathrm{Ga}_{1-x} \mathrm{Mn}_{x} \mathrm{~N}$," Phys. Rev. B 88, 081201(R)

Stefanowicz, W., R. Adhikari, T. Andrearczyk, B. Faina, M. Sawicki, J. A. Majewski, T. Dietl, and A. Bonanni, 2014, "Experimental determination of rashba spin-orbit coupling in wurtzite $n$-GaN:Si," Phys. Rev. B 89, 205201

Stefanowicz, W., D. Sztenkiel, B. Faina, A. Grois, M. Rovezzi, T. Devillers, A. Navarro-Quezada, Tian Li, R. Jakieła, M. Sawicki, T. Dietl, and A. Bonanni, 2010, "Structural and paramagnetic properties of dilute $\mathrm{Ga}_{1-x} \mathrm{Mn}_{x} \mathrm{~N}$," Phys. Rev. B 81, 235210

Stroppa, A., S. Picozzi, A. Continenza, and A. J. Freeman, 2003, "Electronic structure and ferromagnetism of Mndoped group-IV semiconductors," Phys. Rev. B 68, 155203

Suffczyński, J., A. Grois, W. Pacuski, A. Golnik, J. A. Gaj, A. Navarro-Quezada, B. Faina, T. Devillers, and A. Bonanni, 2011, "Effects of $s, p-d$ and $s-p$ exchange interactions probed by exciton magnetospectroscopy in (Ga,Mn)N," Phys. Rev. B 83, 094421

Sugahara, S., K. L. Lee, S. Yada, and M. Tanaka, 2005, "Precipitation of amorphous ferromagnetic semiconductor phase in epitaxially grown Mn-doped Ge thin films," Jpn. J. Appl. Phys. 44, L1426

Sun, Zhi-gang, M. Mizuguchi, and H. Akinaga, 2004, "Au/GaAs magnetoresistive-switch-effect devices fabricated by wet etching," Japan. J. Appl. Phys. 43, 2101

Swagten, H. J. M., A. Twardowski, P. J. T. Eggenkamp, and W. J. M. de Jonge, 1992, " $d-d$ exchange interaction in the diluted magnetic semiconductor $\mathrm{Zn}_{1-x} \mathrm{Co}_{x}$ Se," Phys. Rev. B 46, 188-192

Swalin, R. A., 1970, in Thermodynamics of Solids (Wiley, New York)

Takizawa, H., T. Sato, T. Endo, and M. Shimada, 1987, "High-pressure synthesis and electrical properties of $\mathrm{Mn}_{3} \mathrm{Ge}_{5}$ with $\mathrm{Mn}_{11} \mathrm{Si}_{19}$-type structure," J. Solid State Chem. 68, 234

Takizawa, H., T. Sato, T. Endo, and M. Shimada, 1988, "High-pressure synthesis and electrical and magnetic properties of MnGe and CoGe with the cubic B20 structure," J. Solid State Chem. 73, 40

Takizawa, H., T. Sato, T. Endo, and M. Shimada, 1990, "High pressure synthesis and electrical and magnetic properties of $\mathrm{MnGe}_{4}$ and $\mathrm{CoGe}_{4}, "$ J. Solid State Chem. 88, 384

Takizawa, H., T. Yamashita, K. Uheda, and T. Endo, 2002, "High-pressure synthesis of ferromagnetic $\mathrm{Mn}_{3} \mathrm{Ge}$ with the $\mathrm{Cu}_{3} \mathrm{Au}$-type structure," J. Phys.: Condens. Matter 14, 11147

Talut, G., H. Reuther, A. Mücklich, F. Eichhorn, and K. Potzger, 2006, "Nanocluster formation in Fe implanted GaN," Appl. Phys. Lett. 89, 161909

Tanaka, M., S. Ohya, and Pham Nam Hai, 2014, "Recent progress in III-V based ferromagnetic semiconductors: Band structure, Fermi level, and tunneling transport," Appl. Phys. Rev. 1, 011102

Tanaka, M., M. Yokoyama, Pham Nam Hai, and S. Ohya,
2008, "Properties and functionalities of MnAs/III-V hybrid and composite structures," in Spintronics, edited by T. Dietl, D. D. Awschalom, M. Kaminska, and H. Ohno (Elsevier, Amsterdam) p. 455

Tani, Y., K. Sato, and H. Katayama-Yoshida, 2010, "Materials design of spinodal nanodecomposition in $\mathrm{CuIn}_{1-x} \mathrm{Ga}_{x} \mathrm{Se}_{2}$ for high-efficiency solar energy conversion," Appl. Phys. Express 3, 101201

Tani, Y., K. Sato, and H. Katayama-Yoshida, 2011, "First principles materials design of negative activation energy and transparent conducting sulfides in n-type $\mathrm{CuAl}_{1-x} \mathrm{Sn}_{x} \mathrm{~S}_{2}$ and p-Type $\mathrm{Cu}_{1-x} \mathrm{VCu}_{x} \mathrm{AlS}_{2}$," Appl. Phys. Express 4, 021201

Tani, Y., K. Sato, and H. Katayama-Yoshida, 2012a, "Computational nano-materials design of low cost and high efficiency $\mathrm{Cu}_{2} \mathrm{ZnSn}\left[\mathrm{Se}_{1-x} \mathrm{~S}_{x}\right]_{4}$ photovoltaic solar cells by selforganized two-dimensional spinodal nanodecomposition," Jap. J. Appl. Phys. 51, 050202

Tani, Y., K. Sato, and H. Katayama-Yoshida, 2012b, "Firstprinciples materials design of $\mathrm{CuInSe}_{2}$-based high-efficiency photovoltaic solar cells," Physica B (Amsterdam) 407, 3056

Tani, Y., K. Sato, and H. Katayama-Yoshida, 2012c, "Materials design of $\mathrm{CuIn}_{1-x} \mathrm{Zn}_{0.5 x} \mathrm{Sn}_{0.5 x} \mathrm{Se}_{2}$ for low cost photovoltaic solar cells," J. Non-Cryst. Solids 358, 2420

Tardif, S., S. Cherifi, M. Jamet, T. Devillers, A. Barski, N. Darowski, D. Schmitz, P. Thakur, J. C. Cezar, and N. B. Brookes, 2010a, "Exchange bias in GeMn nanocolumns: The role of surface oxidation," Appl. Phys. Lett. 97, 062501

Tardif, S., V. Favre-Nicolin, F. Lancon, E. Arras, M. Jamet, A. Barski, C. Porret, P. Bayle-Guillemaud, T. Devillers, and M. Rovezzi, 2010b, "Strain and correlation of selforganized $\mathrm{Ge}_{1-x} \mathrm{Mn}_{x}$ nanocolumns embedded in $\mathrm{Ge}(001)$," Phys. Rev. B 82, 104101

Tawara, Y., and K. Sato, 1963, "On the magnetic anisotropy of single crystal of $\mathrm{Mn}_{5} \mathrm{Ge}_{3}$," J. Phys. Soc. Jpn. 18, 773

Tersoff, J., C. Teichert, and M. G. Lagally, 1996, "Selforganization in growth of quantum dot superlattices," Phys. Rev. Lett. 76, 1675

Theodoropoulou, N., A. F. Hebard, S. N. G. Chu, M. E. Overberg, C. R. Abernathy, S. J. Pearton, R. G. Wilson, and J. M. Zavada, 2002a, "Use of ion implantation to facilitate the discovery and characterization of ferromagnetic semiconductors," J. Appl. Phys. 91, 7499

Theodoropoulou, N., A. F. Hebard, M. E. Overberg, C. R. Abernathy, S. J. Pearton, S. N. G. Chu, and R. G. Wilson, 2002b, "Unconventional carrier-mediated ferromagnetism above room temperature in ion-implanted (Ga, Mn)P:C," Phys. Rev. Lett. 89, 107203

Thole, B. T., B. Carra, F. Sette, and G. van der Laan, 1992, "X-ray circular dichroism as a probe of orbital magnetization," Phys. Rev. Lett. 68, 1943

Thomas, L., M. Hayashi, Xin Jiang, R. Moriya, C. Rettner, and S. Parkin, 2007, "Resonant amplification of magnetic domain-wall motion by a train of current pulses," Science 315, 1553

Titov, A., X. Biquard, D. Halley, S. Kuroda, E. BelletAmalric, H. Mariette, J. Cibert, A. E. Merad, G. Merad, M. B. Kanoun, E. Kulatov, and Yu. A. Uspenskii, 2005, "X-ray absorption near-edge structure and valence state of Mn in (Ga,Mn)N," Phys. Rev. B 72, 115209

Tsui, F., L. He, A. Tkachuk, S. Vogt, and Y. S. Chu, 2004, "Evidence for strain compensation in stabilizing epitaxial growth of highly doped germanium," Phys. Rev. B 69, 
081304(R)

Turchi, P. E. A., G. M. Stocks, W. H. Butler, D. M. Nicholson, and A. Gonis, 1988, "First-principles study of ordering properties of substitutional alloys using the generalized perturbation method," Phys. Rev. B 37, 5982

Twardowski, A., H. J. M. Swagten, W. J. M. de Jonge, and M. Demianiuk, 1987, "Magnetic behavior of the diluted magnetic semiconductor $\mathrm{Zn}_{1-x} \mathrm{Mn}_{x}$ Se," Phys. Rev. B 36, 7013-7023

Uchitomi, N., H. Inoue, T. Kato, H. Toyota, and H. Uchida, 2015, "Inhomogeneous distribution of manganese atoms in ferromagnetic $\mathrm{ZnSnAs}_{2}: \mathrm{Mn}$ thin films on $\mathrm{InP}$ revealed by three-dimensional atom probe investigation," J. Appl. Phys. 117, 17B905

Ueda, K., H. Shimizu, and M. Tanaka, 2003, "Magnetooptical kerr effect of semiconductor-based multilayer structures containing a GaAs:MnAs granular thin film," Japan. J. Appl. Phys. 42, L914

van Schilfgaarde, Mark, and O. N. Mryasov, 2001, "Anomalous exchange interactions in III-V dilute magnetic semiconductors," Phys. Rev. B 63, 233205

Venkatasubramanian, R., and D. L. Dorsey, 1993, "Molecularbeam epitaxial growth surface roughening kinetics of $\mathrm{Ge}$ (001): A theoretical study," J. Vac. Sci. Technol. B 11, 253

Venugopal, R., B. Sundaravel, I. H. Wilson, F. W. Wang, and X. X. Zhang, 2002, "Structural and magnetic properties of fe-ge layer produced by Fe ion-implantation into germanium," J. Appl. Phys. 91, 1410

Volnianska, O., and P. Boguslawski, 2010, "Magnetism of solids resulting from spin polarization of p orbitals," J. Phys.: Condens. Matter 22, 073202

Vu, Nguyen Dang, T. Fukushima, K. Sato, and H. KatayamaYoshida, 2014a, "Materials design of interstitial Be-doped ferromagnetic (In,Fe)As," Jpn. J. Appl. Phys. 53, 110307

Vu, Nguyen Dang, T. Fukushima, K. Sato, and H. KatayamaYoshida, 2015, "Computational materials design of LiZnAs, LiZnP-, and LiZnN-based n-type magnetic semiconductors," Jpn. J. Appl. Phys. 54, 053002

$\mathrm{Vu}$, Nguyen Dang, K. Sato, and H. Katayama-Yoshida, 2011, "Giant Peltier effect in self-organized quasi-onedimensional nano-structure in $\mathrm{Cu}-\mathrm{Ni}$ alloy," Appl. Phys. Exp. 4, 015203

Vu, Nguyen Dang, K. Sato, and H. Katayama-Yoshida, 2014b, "Computational materials design of ferromagnetic $\mathrm{Fe}-\mathrm{Cu}$ alloy by phase separation," Jpn. J. Appl. Phys. 53, 073002

Wahl, U., A. Vantomme, G. Langousche, J. G. Correia, L. Peralta, and The ISOLDE Collaboration, 2001, "Direct evidence for implanted $\mathrm{Fe}$ on substitutional Ga sites in GaN," Appl. Phys. Lett. 78, 3217

Wakabayashi, Y. K., Y. Ban, S. Ohya, and M. Tanaka, 2014a, "Annealing-induced enhancement of ferromagnetism and nanoparticle formation in the ferromagnetic semiconductor GeFe," Phys. Rev. B 90, 205209

Wakabayashi, Y. K., S. Ohya, Y. Ban, and M. Tanaka, 2014b, "Important role of the non-uniform Fe distribution for the ferromagnetism in group-IV-based ferromagnetic semiconductor GeFe," J. Appl. Phys. 116, 173906

Wang, K.-Y., M. Sawicki, K.W. Edmonds, R.P. Campion, A.W. Rushforth, A.A. Freeman, C.T. Foxon, B.L. Gallagher, and T. Dietl, 2006, "Control of coercivities in (Ga,Mn)As thin films by small concentrations of MnAs nanoclusters," Appl. Phys. Lett. 88, 022510

Wang, M., R. P. Campion, A. W. Rushforth, K. W. Edmonds, C. T. Foxon, and B. L. Gallagher, 2008, "Achieving high
Curie temperature in (Ga,Mn)As," Appl. Phys. Lett. 93, 132103

Wang, Y., F. Xiu, Y. Wang, X. Kou, A. P. Jacob, K. L. Wang, and J. Zou, 2010, "Mn-rich clusters in gemn magnetic semiconductors: Structural evolution and magnetic property," J. Alloys Compd. 508, 273

Wang, Y., F. Xiu, Y. Wang, J. Zou, W. P. Beyermann, Y. Zhou, and K. L. Wang, 2011, "Coherent magnetic semiconductor nanodot arrays," Nanoscale Res. Lett. 6, 134

Wang, Yutian, Pascal Pochet, Catherine A. Jenkins, Elke Arenholz, Gregor Bukalis, Sibylle Gemming, Manfred Helm, and Shengqiang Zhou, 2014, "Defect-induced magnetism in graphite through neutron irradiation," Phys. Rev. B 90, 214435

Wei, S., W. Yan, Z. Sun, Q. Liu, W. Zhong, X. Zhang, H. Oyanagi, and $\mathrm{Z}$. Wu, 2006, "Direct determination of Mn occupations in $\mathrm{Ga}_{1-x} \mathrm{Mn}_{x} \mathrm{~N}$ dilute magnetic semiconductors by x-ray absorption near-edge structure spectroscopy," Appl. Phys. Lett. 89, 121901

Wellmann, P. J., J. M. Garcia, J.-L. Feng, and P. M. Petroff, 1997, "Formation of nanoscale ferromagnetic MnAs crystallites in low-temperature grown GaAs," Appl. Phys. Lett. 71, 2532

Wellmann, P. J., J. M. Garcia, J.-L. Feng, and P. M. Petroff, 1998, "Giant magnetoresistance in a low-temperature GaAs/MnAs nanoscale ferromagnet hybrid structure," Appl. Phys. Lett. 73, 3291

Weng, H., and J. Dong, 2005, "First-principles investigation of transition-metal-doped group-IV semiconductors: $\mathrm{R}_{x} \mathrm{Y}_{1-x}$ $(\mathrm{R}=\mathrm{Cr}, \mathrm{Mn}, \mathrm{Fe}$; Y = Si, Ge)," Phys. Rev. B 71, 035201

Williamson, G., and W. Hall, 1953, "X-ray line broadening from filed aluminium and wolfram," Acta Metall. 1, 22

Wołoś, A., and M. Kamińska, 2008, "Magnetic impurities in semiconductors," in Spintronics edited by T. Dietl, D. D. Awschalom, M. Kamińska, and H. Ohno (Elsevier, Amsterdam) p. 325

Wołoś, A., M. Piersa, G. Strzelecka, K. P. Korona, A. Hruban, and M. Kamińska, 2009, "Mn configuration in III-V semiconductors and its influence on electric transport and semiconductor magnetism," Phys. Status Solidi C 6, 2769

Woodbury, H. H., and W. W. Tyler, 1955, "Properties of germanium doped with manganese," Phys. Rev. 100, 659

Wróbel, J., T. Dietl, A. Łusakowski, G. Grabecki, K. Fronc, R. Hey, K. H. Ploog, and H. Shtrikman, 2004, "Spin filtering in a hybrid ferromagnetic-semiconductor microstructure," Phys. Rev. Lett. 93, 246601

Xiu, F., Y. Wang, J. Kim, A. Hong, J. Tang, A. P. Jacob, J. Zou, and K. L. Wang, 2010a, "Electric-field-controlled ferromagnetism in high-Curie-temperature $\mathrm{Mn}_{0.05} \mathrm{Ge}_{0.95}$ quantum dots," Nat. Mater. 9, 337

Xiu, F., Y. Wang, X. Kou, P. Upadhyaya, Y. Zhou, J. Zou, and K. L. Wang, 2010b, "Synthesis of high-Curietemperature $\mathrm{Fe}_{0.02} \mathrm{Ge}_{0.98}$ quantum dots," J. Am. Chem. Soc. 132, 11425

Yada, S., Pham Nam Hai, S. Sugahara, and M. Tanaka, 2011, "Structural and magnetic properties of $\mathrm{Ge}_{1-x} \mathrm{Mn}_{x}$ thin films grown on Ge (001) substrates," J. Appl. Phys. 110, 073903

Yamazaki, Y., T. Kataoka, V. R. Singh, A. Fujimori, F.-H. Chang, D.-J. Huang, H.-J. Lin, C. T. Chen, K. Ishikawa, K. Zhang, and S. Kuroda, 2011, "Effect of co-doping of donor and acceptor impurities in the ferromagnetic semiconductor $\mathrm{Zn}_{1-x} \mathrm{Cr}_{x}$ Te studied by soft x-ray magnetic circular dichroism," J. Phys.: Conden. Matter 23 (17), 176002 
Yang, X. L., Z. T. Chen, L. B. Zhao, W. X. Zhu, C. D. Wang, X. D. Pei, and G. Y. Zhang, 2008, "Structural, optical and magnetic properties of $\mathrm{Ga}_{1-x} \mathrm{Mn}_{x} \mathrm{~N}$ films grown by MOCVD," J. Phys. D: Appl. Phys. 41, 245004

Yao, Jia-Hsien, Hsiu-Hau Lin, Yun-Liang Soo, Tai-Sing Wu, Jai-Lin Tsai, Ming-Der Lan, and Tsung-Shune Chin, 2012, "Room-temperature anomalous Hall effect in amorphous Si-based magnetic semiconductor," Appl. Phys. Lett. 100 (9), 092404

Ye, L. H., and A. J. Freeman, 2006, "Defect compensation, clustering, and magnetism in Cr-doped anatase $\mathrm{TiO}_{2}$," Phys. Rev. B 73, 081304(R)

Ye, S., P. J. Klar, Th. Hartmann, W. Heimbrodt, M. Lampalzer, S. Nau, T. Torunski, W. Stolz, T. Kurz, H.A. Krug von Nidda, and A. Loidl, 2003, "Anisotropy of the magnetotransport in ( $\mathrm{Ga}, \mathrm{Mn}) \mathrm{As} / \mathrm{MnAs}$ paramagneticferromagnetic hybrid structures," Appl. Phys. Lett. 83, 3927

Yokoyama, M., T. Ogawa, A. M. Nazmul, and M. Tanaka, 2006, "Large magnetoresistance $(>600 \%)$ of a GaAs:MnAs granular thin film at room temperature," J. Appl. Phys. 99, $08 \mathrm{D} 502$

Yokoyama, M., H. Yamaguchi, T. Ogawa, and M. Tanaka, 2005, "Zinc-blende-type MnAs nanoclusters embedded in GaAs," J. Appl. Phys. 97, 10D317

Yoon, I. T., T. W. Kang, and D. J. Kim, 2006, "Magnetic behavior of $\mathrm{Mn}_{3} \mathrm{GaN}$ precipitates in ferromagnetic $\mathrm{Ga}_{1-x} \mathrm{Mn}_{x} \mathrm{~N}$ layers," Mat. Sci. Eng. B 134, 49

Yu, I.-S., M. Jamet, T. Devillers, A. Barski, P. BayleGuillemaud, C. Beigné, J. Rothman, V. Baltz, and J. Cibert, 2010, "Spinodal decomposition to control magnetotransport in (Ge,Mn) films," Phys. Rev. B 82, 035308

Yu, I.-S., M. Jamet, A. Marty, T. Devillers, A. Barski, C. Beigné, P. Bayle-Guillemaud, and J. Cibert, 2011, "Modeling magnetotransport in inhomogeneous (Ge,Mn) films," J. Appl. Phys. 109, 123906

Zajac, M., R. Doradziński, J. Gosk, J. Szczytko, M. LefeldSosnowska, M. Kamińska, A. Twardowski, M. Palczewska, E. Grzanka, and W. Gȩbicki, 2001, "Magnetic and opti- cal properties of GaMnN magnetic semiconductor," Appl. Phys. Lett. 78, 1276

Zając, M., J. Gosk, E. Grzanka, M. Kamińska, A. Twardowski, B. Strojek, T. Szyszko, and S. Podsiadło, 2003, "Possible origin of ferromagnetism in (Ga,Mn)N," J. Appl. Phys. 93, 4715

Zając, M., J. Gosk, M. Kamińska, A. Twardowski, T. Szyszko, and S. Podsiadło, 2001, "Paramagnetism and antiferromagnetic $\mathrm{d}-\mathrm{d}$ coupling in GaMnN magnetic semiconductor," Appl. Phys. Lett. 79, 2432

Zeng, C., Z. Zhang, K. van Benthem, M. F. Chisholm, and H. H. Weitering, 2008, "Optimal doping control of magnetic semiconductors via subsurfactant epitaxy," Phys. Rev. Lett. 100, 066101

Zhao, Y. J., T. Shishidou, and A. J. Freeman, 2003, "Ruderman-Kittel-Kasuya-Yosida-like ferromagnetism in $\mathrm{Mn}_{x} \mathrm{Ge}_{1-x}, "$ Phys. Rev. Lett. 90, 047204

Zhao, Yu-Jun, and A. Zunger, 2005, "Zinc-blende halfmetallic ferromagnets are rarely stabilized by coherent epitaxy," Phys. Rev. B 71, 132403

Zheng, S., W. Zhu, Y. F. Gao, G. M. Stocks, and Z. Zhang, 2010, "Kinetic Monte Carlo simulations of nanocolumn formation in two-component epitaxial growth," Appl. Phys. Lett. 96, 071913

Zhou, Shengqiang, 2014, "Defect-induced ferromagnetism in semiconductors: A controllable approach by particle irradiation," Nucl. Instrum. Methods Phys. Res. B 326 (0), 55

Zhou, X. H., X. S. Chen, X. G. Guo, L. Z. Sun, Y. L. Sun, and W. Lu, 2004, "Ferromagnetism of 3d-impurities substituted in Ge," J. Magn. Magn. Mat. 284, 253

Zhu, Wenguang, Zhenyu Zhang, and E. Kaxiras, 2008, "Dopant-assisted concentration enhancement of substitutional Mn in Si and Ge," Phys. Rev. Lett. 100, 027205

Zunger, A., 1986, "Electronic structure of 3d transitionatom impurities in semiconductors," in Solid State Physics, Vol. 39, edited by F. Seitz and D. Turnbull (Academic Press, New York) p. 275 